\title{
Unidirectional fibre reinforced geopolymer matrix composites
}

by

Michael Welter

TE WHARE WĀNANGA O TE ŪPOKO O TE IKA A MĀUI

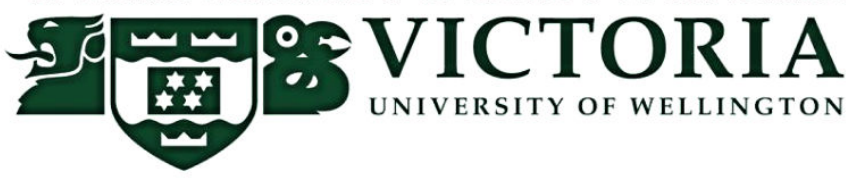

A thesis submitted to the Victoria University of Wellington in fulfilment of the requirements for the degree of

Doctor of Philosophy in Chemistry

Victoria University of Wellington 



\section{Abstract}

Geopolymers have been suggested in the literature as matrix materials for fibre reinforced composites due to a unique combination of low-temperature synthesis and high temperature stability. This study investigated several key aspects of fibre reinforced geopolymer matrix composites in order to improve the basic knowledge of these materials. It was demonstrated that geopolymer matrix composites show great potential as fire-resistant materials for near room temperature applications. In particular, basalt fibre composites were of great interest due to their comparatively low cost and good mechanical performance. Microstructural investigations indicated that basalt fibres can potentially be used in geopolymer matrices up to $600^{\circ} \mathrm{C}$. However, the success of the application of geopolymer matrix composites at higher temperatures is seen as critical and depends on further development of suitable matrices.

Several compositions within a sodium-metahalloysite model matrix system were evaluated in order to identify a suitable formulation for composite fabrication. An average compressive strength of $\sim 79 \mathrm{MPa}$ and flexural strength and modulus of $\sim 10$ $\mathrm{MPa}$ and $8.5 \mathrm{GPa}$, respectively, were achieved for the best batch of the main matrix composition. By optimising the matrix composition, the mechanical properties could be significantly improved, achieving an extremely high maximum compressive strength value of $145 \mathrm{MPa}$. Issues with reproducibility and the influence of various aspects of the fabrication process are discussed. 
The room temperature flexural properties of unidirectional fibre reinforced composite bars with basalt, carbon and alumina fibres were investigated. Besides the fibre type, the effects of several other parameters including fibre sizing, matrix strength, span-to-depth ratio and specimen dimensions on the flexural properties and the failure behaviour of the composites were studied. Significant improvements to the mechanical properties were achieved with all fibre types. However, the mechanical behaviour was highly influenced by the elastic modulus of the fibre. Furthermore, it was shown that the composite properties were affected by the overall sample dimensions, the testing span and the mixing time of the geopolymer binder. The alumina fibre composites achieved the highest flexural stress with a maximum value of $470 \mathrm{MPa}$ and a fibre content of $\sim 30$ vol.-\%. Basalt and carbon fibre composites showed maximum flexural strength values around $200 \mathrm{MPa}$. Although all composite types displayed considerable post-fracture strength, only the basalt composites failed in tensile mode. The applicability of the weak matrix composites (WMC) concept to describe the mechanical behaviour of geopolymer matrix composites was discussed.

The fibre-matrix interactions were analysed between room temperature and $1000^{\circ} \mathrm{C}$ by means of electron microscopy, EDS and x-ray diffraction. All fibres were found to be chemically stable under the highly alkaline conditions of the geopolymer synthesis and showed no significant reaction with the geopolymer matrix at room temperature. The results indicate that basalt fibre composites may be used up to $600^{\circ} \mathrm{C}$ without significant degradation of the fibre. The heating of the carbon fibre composites to $600^{\circ} \mathrm{C}$ had drastic effect on the strength and integrity of the composite, in particular, when using sized carbon fibres. The alumina fibres showed good wetting and bonding behaviour but otherwise little reaction with the matrix even after heating to $1000^{\circ} \mathrm{C}$. 


\section{Acknowledgements}

I would like to thank my supervisor Prof. Ken MacKenzie for offering me the opportunity to undertake my $\mathrm{PhD}$ study in New Zealand, for continuously encouraging me in my own work and for the trust he put in me during these years.

I am also very thankful to Dr. Martin Schmuecker for accepting the role as cosupervisor as well as providing some of the fibre materials for this study. Despite being half way around the world, his input and support has been invaluable.

I would also like to thank the MacDiarmid Institute for providing the funding for this project. Additional funding provided through the Curtis-Gordon Scholarship, the Faculty Research Grant and the Victoria University Submission Scholarship was also greatly appreciated.

A big thank you goes to my past and present group members, in particular Brian O'Leary for all the lively discussions we had. I would also like to thank all my fellow lab members for their support and friendship over the years. In particular, I would like to thank Matthias Herzog, Martin Klimsa and Christin Gaedtke who have become great friends.

I am also very grateful to David Flynn for all the support with the electron microscopy and to Manu, Nick and Allen from the workshop who fabricated the 
mixer and the moulds that were used in this study. I would also like to acknowledge Ian Minchington for letting me use the mechanical testing equipment at IRL.

I cannot thank my family enough for all their support and encouragement over the past years. I am equally indebted to Cushla, John and the rest of Roses family who have welcomed me into their family a long time ago and have since always been there for me.

My final and biggest thank you goes to my partner Rose who has always been there for me during many good and but also hard times. Without her endless love, support and encouragement, the completion of this thesis would not have been possible. 


\section{Table of contents}

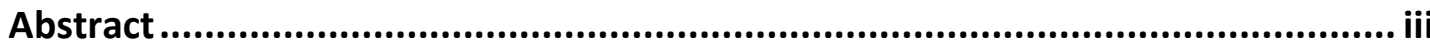

Acknowledgements...............................................................................................

Table of contents ........................................................................................... vii

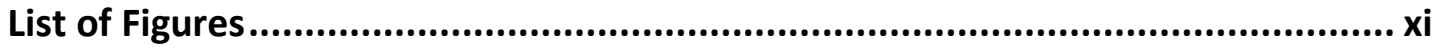

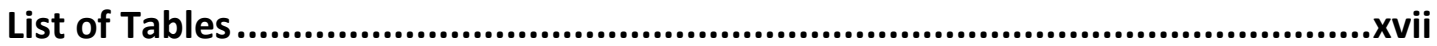

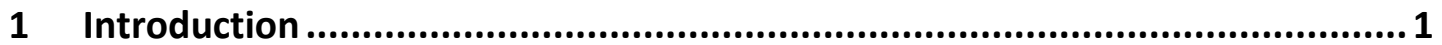

2 Background and literature review............................................................ 7

2.1 Introduction to composite materials ................................................... 7

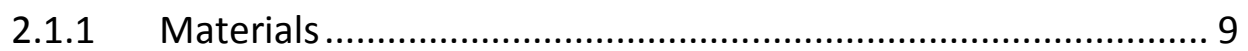

2.1.2 Conventional fabrication methods ...................................... 12

2.1.3 Mechanical failure behaviour of ceramic matrix composites .. 14

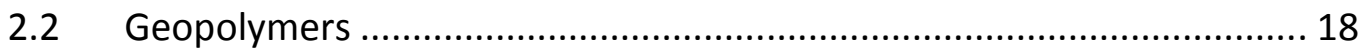

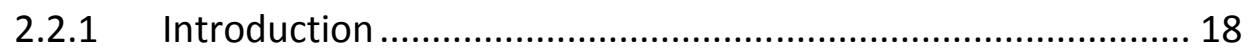

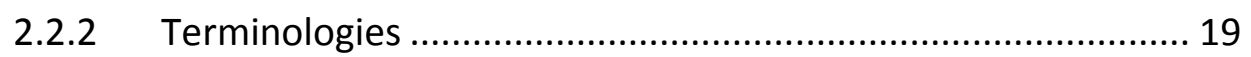

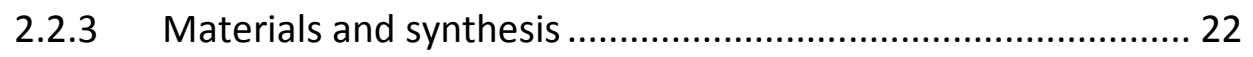

2.2.4 Geopolymerisation process ............................................... 25

2.2.5 Chemical and structural aspect of metakaolin geopolymers ... 25

2.2.6 Properties ................................................................... 28

2.3 Geopolymer matrix composites ...................................................... 31

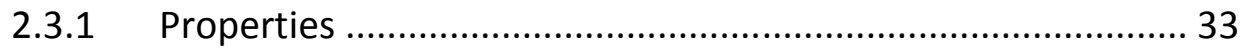

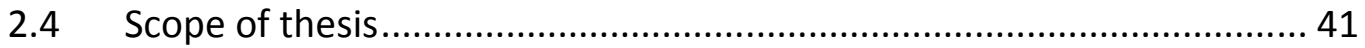




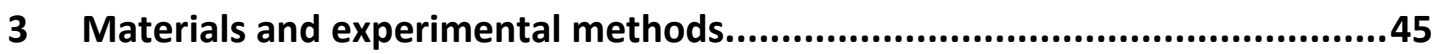

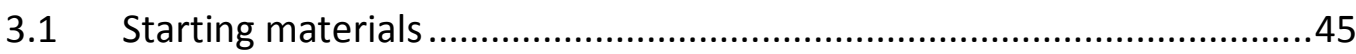

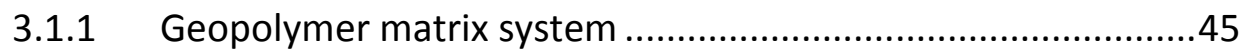

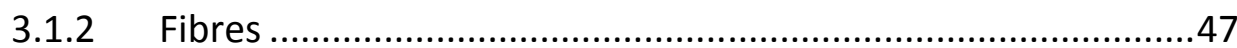

3.2 Composition and processing of geopolymer binders ...........................48

3.3 Fabrication of unreinforced specimens ...............................................50

3.4 Fabrication of fibre reinforced specimens .......................................52

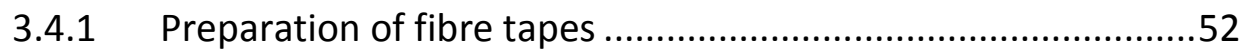

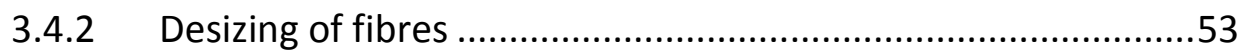

3.4.3 Fabrication of unidirectional composite bars ..........................54

3.4.4 Nomenclature for composite samples ...................................56

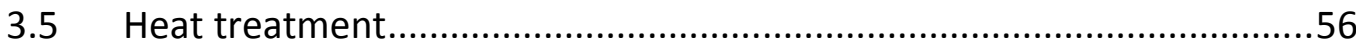

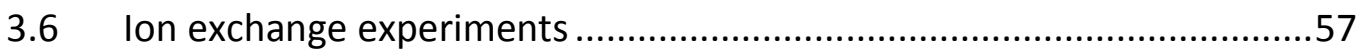

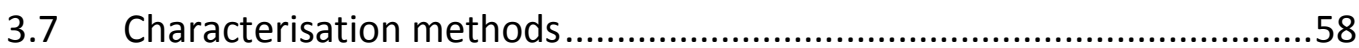

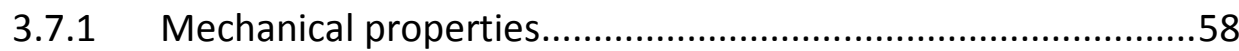

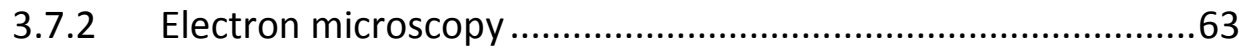

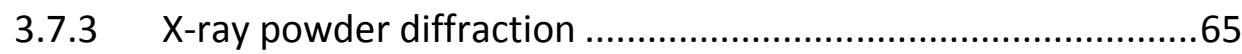

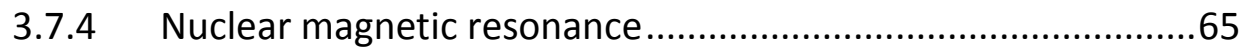

3.7.5 Thermal analysis - TGA/DSC ................................................66

4 Characterisation of geopolymer matrices ................................................67

4.1 Fabrication process and initial investigations ....................................67

4.1.1 Mixing, viscosity and use-time of geopolymer binders............67

4.1.2 Optimisation of the fabrication process...................................69

4.1.3 Drying process .................................................................... 70

4.2 Mechanical and physical properties .................................................... 73

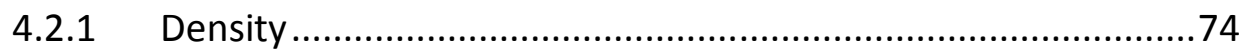

4.2.2 Splitting-tensile strength ................................................. 74

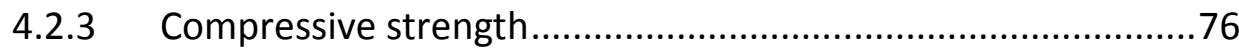

4.2.4 Flexural strength and modulus.............................................79

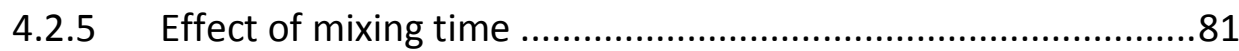

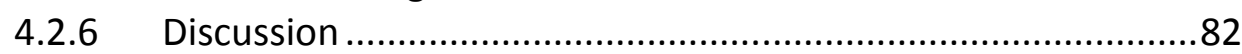

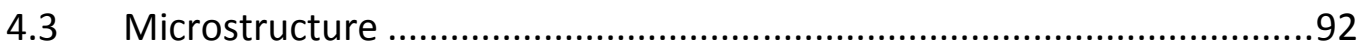

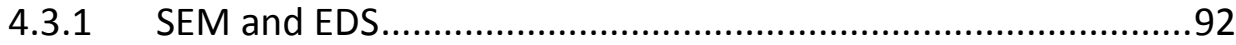

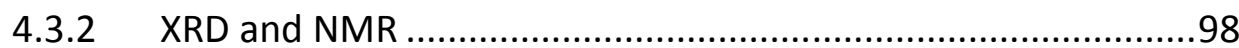

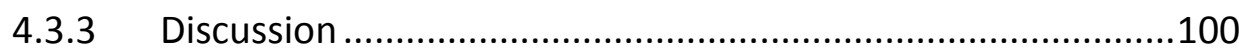




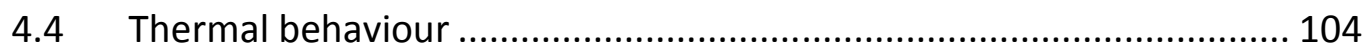

4.4.1 Microstructural evolution and crystallisation ....................... 104

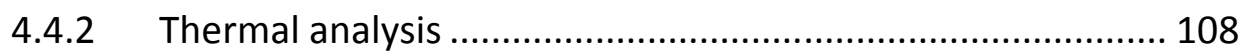

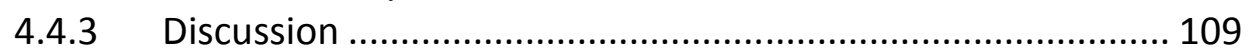

4.5 Discussion on the reproducibility of mechanical strength with regard to fabrication process and microstructure............................. 110

5 Mechanical properties of unidirectional composites .............................. 115

5.1 Effects of fibre type and fabrication process on the flexural properties and stress-strain behaviour............................................ 116

5.1.1 General observations and fabrication process..................... 116

5.1.2 Fibre type ...................................................................... 119

5.1.3 Fibre distribution in composite bars.................................... 125

5.2 Other factors influencing the flexural properties ............................. 126

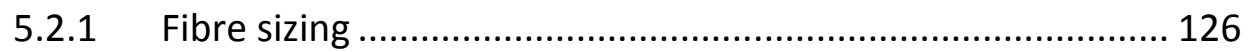

5.2.2 Matrix composition and strength ...................................... 128

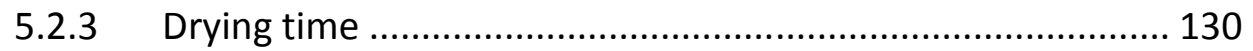

5.2.4 Specimen dimensions and span-to-depth ratio ................... 131

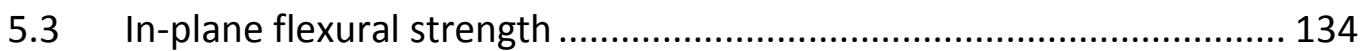

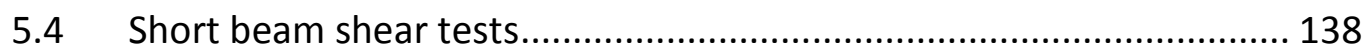

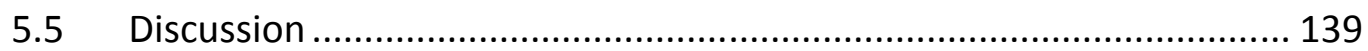

5.5.1 The influence of fabrication process and processing

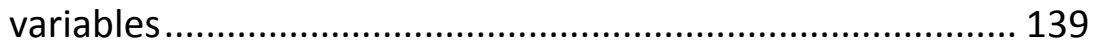

5.5.2 The effect of fibre type on the mechanical properties .......... 142

5.5.3 Other factors influencing the mechanical properties ............ 146

5.5.4 Failure behaviour of geopolymer matrix composites under flexural loading........................................................ 149

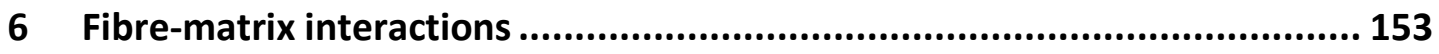

6.1 Fibre Fibre-matrix interaction at room temperature .......................... 153

6.1.1 Chemical stability and desizing of fibres ............................. 153

6.1.2 Microstructural analysis of geopolymer composites ............. 154

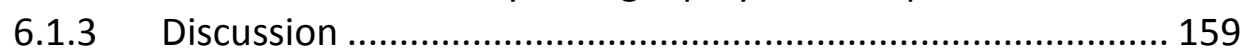

6.2 Fibre-matrix interaction at elevated temperatures ............................ 160

6.2.1 Basalt fibre composites...................................................... 160

6.2.2 Carbon fibre composites.................................................... 172

6.2.3 Alumina fibre composites.................................................. 174

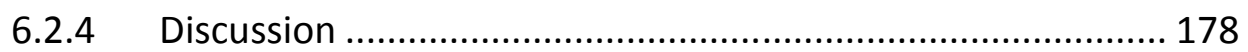


7 Geopolymer composites as precursors for CMCs - General considerations and initial results........................................................... 185

7.1.1 Ion exchange experiments ...............................................185

7.1.2 Discussion .....................................................................188

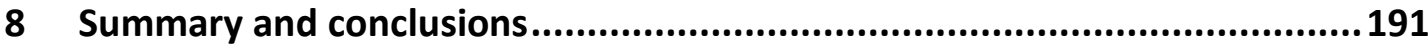

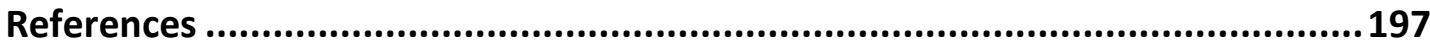




\section{List of Figures}

Figure 2.1: Boundary conditions of the relative fracture energy in relation to the elastic mismatch of fibre and matrix for non-brittle failure behaviour after He and Hutchinson [36], diagram from [37]

Figure 2.2: Energy dissipating mechanisms in fibre reinforced WIC-CMCs, after [40]..... 16

Figure 2.3: Proposed structural model for metakaolin geopolymers by Rowles et al. [91] 27

Figure 2.4: Stress-strain curves of fabric (c, $d ; v_{f}=60$ vol.- $\left.\%\right)$ and unidirectional (UD) $\left(a, b ; V_{f}=70\right.$ vol.-\%) fibre reinforced geopolymer composites after Foerster $[3,5]$.

Figure 2.5: Residual strength and modulus of 3 and $6 \mathrm{~mm}$ thick $3 \mathrm{k}$ carbon fabric reinforced geopolymer composites after heat treatment in air for one hour, after Foden [9] and Lyon et al. [7]

Figure 2.6: Stress-strain curves of geopolymer and unidirectional carbon fibre reinforced geopolymer composites before and after heat treatment, after He et al. [13]. The fibre volume content of unheated composites is $20-25 \%$.

Figure 2.7: Fracture surfaces of unidirectional carbon fibre reinforced geopolymer composites: (a) before heat treatment, (b) heated at $1100^{\circ} \mathrm{C}$, (c) heated at $1400^{\circ} \mathrm{C}$, (d) interface reaction after heating at $1400^{\circ} \mathrm{C}$ in argon for 90 minutes, after He et al. [13]

Figure 3.1: Typical DTA curve (a) and particle size distribution (b) of NZCC Halloysite Premium as specified by supplier

Figure 3.2: Morphology of dehydroxylated halloysite after calcination at $600^{\circ} \mathrm{C}$ over night and screening on $353 \mu \mathrm{m}$ sieve: a) high and b) low resolution SEM micrograph.....

Figure 3.3: Schematic presentation of the mixer design developed and used in this work 50

Figure 3.4: Moulds used for the fabrication of a) unreinforced and UD composite bars b) unreinforced cylinders and c) unreinforced pellets 
Figure 3.6: Schematic of standard heating programme showing heating rates and annealing steps

Figure 4.1: Typical weightloss of pellet specimens of compositions M1 - M4 during the drying process under ambient conditions

Figure 4.2: Visibility of micro- and macrocracks in M1 pellets before (a) and after (b) wiping the sample surfaces with IPA. The appearance of several microcracks in the seemingly crack free samples 1 and 4 are clearly visible.

Figure 4.3: Initial evaluation of the mechanical strength of the hand mixed compositions M1-M4 by means of splitting-tensile strength measurements

Figure 4.4: Splitting-tensile strengths of selected sample series of composition M1 subjected to different mixing, curing and drying parameters. Several different machining and grinding preparations were tested on the four hand mixed sample batches (not further specified here). All other sample series were mechanically machined in the same way

Figure 4.5: High magnification images of fracture surfaces, displaying typical microstructures of well reacted areas of geopolymer compositions M1 - M5

Figure 4.6: SEM analysis of fracture surface of M5 bar specimen: a) low magnification image showing typical irregular surface pattern of elevated and lowered areas, largely consistent with the lighter coloured areas in backscatter image (b); c) higher magnification image showing microstructural difference between lighter area and main phase. EDS spot analyses indicate the different composition with regard to the $\mathrm{SiO}_{2} / \mathrm{Al}_{2} \mathrm{O}_{3}$ ratio of the two phases; d) EDS elemental map of image b). The blue coloured areas indicate a comparatively lower sodium and higher aluminium concentration compared to the relatively homogeneous distribution of $\mathrm{Na}, \mathrm{Si}$ and $\mathrm{Al}$ in the surrounding main phase

Figure 4.7: Phase inhomogeneities on fracture surface of composition M2. Arrows indicate microstructural areas as shown in Figure 4.5-M2

Figure 4.8: EDS mapping of various areas of fracture surface of $M 2$ bar specimen: $(a, b)$ low magnification image and EDS map showing a very inhomogeneous sodium distribution. The needle structures $(c)$ are identified as very sodium rich phases by the EDS map (d)

Figure 4.9: X-ray diffraction patterns for geopolymer compositions M1 - M5 and metahalloysite... 98

Figure 4.10: ${ }^{27} \mathrm{Al}$ and ${ }^{29} \mathrm{Si}$ MAS-NMR spectra of geopolymer compositions M1 - M5 .99

Figure 4.11: Microstructure and formation of small rod-like shapes in sample $\mathrm{M} 1-600^{\circ} \mathrm{C}$ 105

Figure 4.12: Microstructural appearance of samples $\mathrm{M} 1$ (a) and $\mathrm{M} 5$ (b) after heating to $1000^{\circ} \mathrm{C} \ldots . .105$

Figure 4.13: Low resolution SEM image of sample $M 5-1000^{\circ} \mathrm{C}$ revealing a very inhomogeneous and porous structure 
Figure 4.14: $X$-ray diffraction patterns for compositions $M 1$ and $M 5$ after heating up to $1000^{\circ} \mathrm{C} \ldots .107$

Figure 4.15: Weighloss and DSC curves for composition M1 .108

Figure 5.1: Stress-strain curves recorded for the alumina, carbon and basalt composites (top to bottom) presented in Table 5.1

Figure 5.2: Typical stress-strain behaviour of unidirectional basalt, carbon and Nextel 610 alumina fibre reinforced geopolymer matrix composites.

Figure 5.3: Typical macroscopic failure behaviour of UD composite bars made with basalt (top), carbon (middle) and Nextel 610 alumina fibres (bottom)

Figure 5.4: Representative stress-strain curves of basalt-carbon and basalt-boron fibre composites in comparison to basalt fibre composites.

Figure 5.5: Typical fibre distribution in (a) basalt, (b, d) carbon and (c) Nextel 610 fibre composites

Figure 5.6: Comparison of typical stress-strain behaviour of composites made from sized and desized carbon and Nextel 610 fibres

Figure 5.7: Effect of matrix strength on the stress-strain behaviour of basalt and carbon fibre composites

Figure 5.8: Comparison of stress-strain curves of basalt fibre composites after seven and 28 days drying time

Figure 5.9: Effect of sample thickness and span-to-depth ratio on the stress-strain behaviour of basalt fibre composites

Figure 5.10: Typical macroscopic failure observed for UD basalt fibre composites depending on sample thickness and span-to-depth ratio: s/d 32:1, $100 \mathrm{~mm}$ span (top), $\mathrm{s} / \mathrm{d} \sim 16: 1,50 \mathrm{~mm}$ span (middle) and $\mathrm{s} / \mathrm{d} \sim 16: 1,100 \mathrm{~mm}$ span (bottom).

Figure 5.11: Stress-strain curves recorded for in-plane and out-of-plane flexural testing of basalt (left) and carbon (right) composites.

Figure 5.12: Typical in-plane failure patterns observed for basalt $(a-c)$ and carbon $(d-f)$ fibre composites.

Figure 6.1: SEM micrographs of basalt fibres before (left) and after (right) exposure in concentrated $\mathrm{NaOH}$ solution at $40^{\circ} \mathrm{C}$ for $150 \mathrm{~h}$. No form of corrosive attack on the fibre surface is observed. The removal of fibre sizing is typical for all investigated types of sized fibres

Figure 6.2: Adhesion between M1 matrix and a) basalt, b) Nextel 610, c) Nextel 610-DS and d) carbon/carbon-DS fibres

Figure 6.3: Nextel 610-DS fibre at the transition point between a thick matrix adhesion layer and clean fibre surface 
Figure 6.4: SEI and backscattered images of the polished surface of the M1-Basalt composite and corresponding EDS line scan profile across the fibre-matrix interface.....

Figure 6.5: SEI and backscattered images of the polished surface of the M1-N610 composite and corresponding EDS line scan profile across the fibre-matrix interface.

Figure 6.6: X-ray diffraction patterns of basalt fibre, M1-Basalt composite and M1 reference sample

Figure 6.7: Fracture surfaces of basalt fibre composites after heat treatment at a) $600^{\circ} \mathrm{C}$, b) $800^{\circ} \mathrm{C}$ and c) $1000^{\circ} \mathrm{C}$

Figure 6.8: SEM micrographs of M1-Basalt composites heated to $800^{\circ} \mathrm{C}$ showing an embedded fibre in the fractured composite and the matrix microstructure in an empty fibre channel. The microstructural appearance displayed in both images is equally representative for the $600^{\circ} \mathrm{C}$ samples

Figure 6.9: X-ray diffraction patterns of M1-Basalt composites heated to 600 and $800^{\circ} \mathrm{C}$ for $1 \mathrm{~h} \ldots 164$

Figure 6.10: SEM backscattered images of the fracture surface of the M1-Basalt composite heated at $800^{\circ} \mathrm{C}$ for $1 \mathrm{~h}$. EDS line scan analysis indicates the formation of an iron-rich inner fibre reaction zone.

Figure 6.11: SEM images of fracture surfaces of the M1-Basalt composite after heating to $1000^{\circ} \mathrm{C}$. The images show the formation of large pores within the fibre; inhomogeneous, dense and porous matrix areas and fibre deformation within the composite

Figure 6.12: SEM backscattered images of the polished surface of the M1-Basalt composite after heating to $1000^{\circ} \mathrm{C}$ showing fibre shape accommodation and crystallisation within the fibre and EDS line scan profile across the fibre-matrix interface (see also Figure 6.13 for EDS elemental maps)

Figure 6.13: EDS elemental maps corresponding to micrograph shown in Figure 6.12(b) (element concentration increases from dark to bright colour)

Figure 6.14: Backscattered SEM image of fibre-matrix interface of the M1-Basalt composite after heating to $1000^{\circ} \mathrm{C}$ at higher resolution with overlay of EDS line scan profile for Fe and $\mathrm{Mg}$ and corresponding EDS elemental maps (element concentration increases from dark to bright colour)

Figure 6.15: Backscattered SEM image of fractured basalt fibres heated to $1000^{\circ} \mathrm{C}$ for $1 \mathrm{~h}$ 170

Figure 6.16: Comparison of $x$-ray diffraction patterns of basalt fibre, M1 matrix and M1-Basalt composite heated to $1000^{\circ} \mathrm{C}$ for $1 \mathrm{~h}$

Figure 6.17: Photographs of M1-C and M1-C-DS composite samples heated to 600 and $800^{\circ} \mathrm{C}$

Figure 6.18: SEM micrographs of carbon fibre composite samples heated to $600^{\circ} \mathrm{C}$ : a) fracture piece of sample M1-C, b) fracture surface of sample M1-C-DS, c) and d) showing different microstructural appearances of the matrix in fibre channels and fibre degradation (c) representative for both samples. 
Figure 6.19: Typical appearance of fibres on the fracture surface of a) M1-N610 and b) M1-N610-DS composite samples after heating to 600 and $800^{\circ} \mathrm{C}$. No change in the microstructural appearance of the composite samples was observed between the two temperatures.

Figure 6.20: Examples of different microstructural appearances of fracture surfaces along the fibre orientation representative for both M1-N610 and M1-N610-DS composites after heating to 600 and $800^{\circ} \mathrm{C}$. No change in the microstructure was observed between 600 and $800^{\circ} \mathrm{C}$

Figure 6.21: SEM micrographs (a) and EDS elemental map (b) of fibres on the fractured surface of M1-N610-DS after heating to $1000^{\circ} \mathrm{C}$

Figure 6.22: Backscattered SEM image with corresponding EDS elemental map and line scan profile across the fibre-matrix interface of the polished surface of the M1-N610 composite heated to $1000^{\circ} \mathrm{C}$

Figure 7.1: Typical appearance of ion exchanged bar specimens after firing at 1000 and $1100^{\circ} \mathrm{C} \ldots 186$

Figure 7.2: EDS maps on polished surfaces showing sodium distribution in ion exchanged unreinforced bar (a, b) and Nextel 610 fibre reinforced composite (c, d) specimens with $\mathrm{M} 1$ matrix after heating to $1000^{\circ} \mathrm{C}$ (Na concentration increases from black to white colour as indicated by sidebar)

Figure 7.3: Comparison of $x$-ray diffraction patterns of ion exchanged $M 1$ powder samples, bar pieces and M1-N610 composite samples after firing at 1000 and $1100^{\circ} \mathrm{C}$ 


\section{List of Tables}

Table 2.1: Formal representation of the three fundamental sialate units

Table 2.2: Reported mechanical properties and compositions of metakaolin geopolymers used as composite matrices....

Table 2.3: Maximum flexural properties of selected fibre fabric reinforced geopolymer composites at room temperature.

Table 2.4: Maximum flexural properties of selected unidirectional fibre reinforced geopolymer composites at room temperature

Table 2.5: Comparison of various mechanical properties of unidirectional and $3 \mathrm{k}$ fabric carbon fibre reinforced geopolymer composites at room temperature, after Foden [9]

Table 3.1: Chemical analysis of dehydroxylated halloysite ..... 46

Table 3.2: Investigated fibres and relevant properties as specified by the supplier 47

Table 3.3: Chemical analysis of basalt fibre 48

Table 3.4: Component mixture and estimated molar ratios of investigated compositions..... 49

Table 3.5: Instrument settings for ${ }^{29} \mathrm{Si}$ and ${ }^{27} \mathrm{Al}$ MAS NMR...... 66

Table 4.1: Bulk densities of compositions M1 - M5 measured on small cylinder specimens.

Table 4.2: Variability of compressive strength between different batches (including the strongest and weakest) of composition M1 all prepared by the same fabrication process and conditions

Table 4.3: Average compressive strength of the strongest batch of all matrix compositions 78

Table 4.4: Influence of drying time on the compressive strength of composition M1 79 
Table 4.5: Variability of flexural properties between different batches of composition M1 all prepared by the same fabrication process and conditions ( \pm standard deviation).....

Table 4.6: Comparison of the flexural properties of the strongest batch of compositions

M1 - M5

Table 4.7: Comparison between chemical composition and mechanical properties

Table 4.8: Comparison of mechanical properties of compositions $M 1$ and M5 with other metakaolin geopolymer matrices reported in the literature

Table 5.1: Flexural properties datasets of three exemplary composite types (M1-Basalt, M1-Carbon, and M1-N610) to show the general influence of the order of fabrication on the flexural strength.

Table 5.2: Effect of fibre type on the flexural properties of geopolymer matrix composites

Table 5.3: Flexural properties of mixed basalt/carbon and basalt/boron fibre reinforced geopolymer composites

Table 5.4: Effect of fibre sizing on the flexural properties of geopolymer matrix composites

Table 5.5: Effect of matrix strength on the flexural properties of basalt and carbon fibre composites

Table 5.6: Effect of drying time on the flexural properties of basalt fibre composites

Table 5.7: Effect of specimen thickness and span-to-depth ratio on the flexural properties of basalt fibre composites

Table 5.8: Comparison of in-plane and out-of-plane flexural properties of basalt and carbon fibre composites 


\section{Chapter 1}

\section{Introduction}

With advances in technological development, an increasing number of today's applications call for materials with improved properties that cannot always be met by conventional bulk materials. In that regard, composite materials offer a major improvement towards the optimisation of material properties. In the most general sense, composites can be described as multiphase materials consisting of at least two distinct components. The interaction between these components results in a material with properties that cannot be obtained with any of the constituents alone. There are many different types of composites. However, for the purpose of this study, only artificial structural composites, in particular continuous fibre reinforced composites, are considered. Two main constituents of structural composites can usually be distinguished. These are generally referred to as the matrix and the reinforcement.

One of the main advantages of composite materials is the nearly unlimited combination of different materials. This opens the possibility to tailor the composite properties to meet specific application requirements. In many cases, the efforts of improving material properties are focused on improvements of the specific mechanical properties, making materials stronger, stiffer and tougher but also lighter. However, besides the mechanical performance other factors such as durability, the 
required temperature stability and particularly cost play an important role in the development of new materials.

Three main classes of structural composite materials are generally distinguished according to the nature of their matrix, i.e. polymer matrix composites (PMCs), metal matrix composites (MMCs) and ceramic matrix composites (CMCs). Currently, the most common types of fibre composites are based on glass and carbon fibre reinforced polymer matrices [1]. These composites combine high strength with light weight and relatively easy and cost-effective fabrication methods at moderately low temperatures. However, the organic nature of the matrix limits their operating temperature to about $200^{\circ} \mathrm{C}$. Flammability and the development of toxic fumes and smoke may also restrict their application in some fields. For applications beyond the limitations of PMCs, metal or ceramic-based materials must be used. Ceramics generally exhibit very desirable properties such as relatively low density, high strength and stiffness, chemical stability and superior high temperature properties. However, the inherently brittle nature of ceramic materials is a major limitation for their widespread use in structural and engineering applications. This problem can be largely overcome by the incorporation of inorganic fibres to increase the toughness of the matrix and introduce a quasi-ductile, graceful failure behaviour. But despite extensive research efforts over the past decades the commercial relevance of fibre reinforced CMCs, and also MMCs, is still relatively small compared to PMCs. This can be, at least partly, attributed to the much higher overall costs of CMCs due to more complex processing requirements, higher material costs and much higher manufacturing temperatures. The high costs of CMCs may be justified in some highly demanding, high temperature applications where a specific properties profile leaves the material selection without alternative. However, for less demanding applications at temperatures below around $600-800^{\circ} \mathrm{C}$ their application becomes increasingly uneconomical. Since the application temperature of polymer based composites is limited to about $200^{\circ} \mathrm{C}$, this leaves only a small number of materials, mostly metal-based, for applications in the temperature range from about $200-$ $800^{\circ} \mathrm{C}$. Several factors, however, such as higher density, insufficient corrosion resistance, electrical conductivity or costs may make the use of metals unfavourable 
for certain applications. Therefore, new inorganic, non-metal materials that can essentially combine high strength, low density and reasonably high temperature stability in a cost-effective way for applications in this mid-temperature range would be of considerable interest.

Inorganic aluminosilicate polymers, commonly referred to as geopolymers, may offer an alternative to common ceramic and polymer matrix materials and are not subjected to some of the drawbacks of these materials. Geopolymers are synthetic inorganic compounds, generally made by reaction of aluminosilicate sources and alkali activation solutions at high $\mathrm{pH}$ and near ambient temperatures. The initially viscous geopolymer binders cure and harden in similar fashion to organic thermosetting resins to form a generally amorphous three-dimensional solid material with ceramic-like properties. Typically, geopolymers are characterised by low density, reasonable mechanical strength and good temperature resistance up to $1000^{\circ} \mathrm{C}$. However, they are also inherently brittle. Although the mechanical properties of geopolymers are much more similar to ordinary Portland Cement (OPC) and some organic polymers rather than common engineering ceramics such as $\mathrm{Al}_{2} \mathrm{O}_{3}$, mullite or $\mathrm{SiC}$, the unique combination of properties and low temperature processing has considerable potential in developing cost-effective inorganic composite materials. Thus, they offer an economical alternative to conventional $\mathrm{CMCs}$ for applications in a mid-temperature range up to $\sim 800^{\circ} \mathrm{C}$, effectively bridging the gap between existing PMC and CMC materials. Furthermore, geopolymer composites may also be of interest as precursors for the fabrication of true CMCs due to the typical transformation of geopolymers into crystalline ceramic phases at higher temperatures.

The idea to use geopolymers as a matrix material for fibre reinforced composites was first investigated in the 1980s with the objective to fabricate moulding tools and patterns for the plastic processing industry [2]. Subsequently, it has been shown that the mechanical properties of geopolymers can be significantly improved by the incorporation of inorganic fibres such as carbon, silicon carbide ( $\mathrm{SiC}$ ), alumina, aluminosilicate, basalt and glass fibres [3-30]. However, despite the significant 
potential of geopolymer matrix composites for structural applications suggested in the existing literature, these materials have so far received relatively little attention and have only attracted applications in some niche markets. In particular with regard to continuous fibre reinforced geopolymer composites [3-19], the published literature and property database remain scarce. One of the main reasons for this may be associated with a general scepticism against geopolymer science and technology in the past. However, geopolymer technology has established itself in recent years as a growing area of scientific interest and significant advances towards a better understanding of geopolymers have been made since the first studies on geopolymer composites. Thus, the full potential of these materials has yet to be determined.

In the present study, several key aspects of unidirectional fibre reinforced geopolymer matrix composites were investigated with the aim to improve the basic knowledge of these materials and to evaluate their general potential as cost-efficient composite materials for structural applications. One of the main problems of geopolymers is the often difficult comparability between different studies due to the use of different starting materials, matrix compositions, processing and fabrication techniques, testing methods and parameters, amongst others. Therefore, instead of focusing on one particular aspect, a broad objective was chosen for this work to allow for the direct comparison of the effects of a number of different variables on the composite properties in one comprehensive study. However, that also means that the depth to which individual parameters could be investigated was limited within the scope of this thesis.

The following four main aspects were investigated:

(i) characterisation of a model geopolymer matrix system

(ii) investigation of the effects of various parameters on the mechanical properties and failure behaviour of unidirectional fibre reinforced composites at room temperature

(iii) analysis of fibre-matrix interactions up to $1100^{\circ} \mathrm{C}$ 
(iv) initial ion exchange experiments and general considerations regarding the use of geopolymer matrix composites as precursors for CMCs

A more detailed outline of the scope of this study is presented in chapter 2.4. 


\section{Chapter 2}

\section{Background and literature review}

The following chapter provides a brief introduction to fibre reinforced composite materials. Furthermore, relevant information on geopolymers and a detailed literature review of fibre reinforced geopolymer composites are presented in order to establish the basic background for this study.

\subsection{Introduction to composite materials}

The technology of composite materials is one of the most important advances in materials development. In fact, many of today's advanced technologies would be unimaginable without composite materials. However, the idea of composites is not new and many materials of natural and artificial source are effectively composites [31]. This raises the question of what composites actually are and how they are defined. The common conception of what is considered a composite may vary considerably between different fields of research. In the most general way, a composite can be described as a material that consists of two or more dissimilar constituents. This definition, however, is quite flexible and allows the term composite to be used to describe almost anything, especially when considering at a close enough level $[31,32]$. For the purpose of modern materials engineering, 
composites are commonly defined as materials that: (i) consist of at least two chemically and physically distinct phases, (ii) are artificially bonded together and (iii) produce a structural material with enhanced properties that cannot be obtained with either of these phases taken separately [33]. In practice, the involved phases can usually be distinguished into a continuous phase called the matrix and a strong disrupted phase usually referred to as the reinforcement. Several types of reinforcements can be distinguished: (i) continuous/long fibres, (ii) discontinuous/short fibres, (iii) whiskers (small elongated single crystals) and (iv) platelets and particles. Nevertheless, in the context of engineering materials the term composite is mainly used in reference to fibre reinforced materials [32]. One of the advantages of using materials in fibrous form is their much higher strength compared to respective bulk forms. This is due to the small size of the fibres and the accompanying reduction of critical defects in the material structure. However, since the use of materials in fibre form is not applicable for most engineering purposes they need to be bonded together by a matrix to form a strong and stiff solid [33]. Although the matrix is generally the weaker phase and dilutes the properties of the fibres to some degree, the matrix enables load transfer to the fibres and also protects them from abrasion and environmental attack [32]. Thus, both the matrix and the fibre properties play a fundamental role in the performance of composites.

The concept of composite materials has been employed by man for thousands of years. One of the oldest examples of artificial, man-made composite materials are straw reinforced mud bricks for building purposes. A more modern example is the use of steel rods or mesh to reinforce concrete structures, a practice which finds its origins in the mid- $19^{\text {th }}$ century and may be seen as the origin of composite research. Nevertheless, composite materials did not become a distinct discipline of materials research until around the 1960s [34]. The developments at that time were strongly driven by the advances in aerospace technology and composites were, for the most part, only considered for high-performance applications, thus making them quite exclusive. Today, composites are widely used and applications range from high tech aerospace constructions to body parts for cars, trains and aircrafts; high temperature, corrosion, oxidation and/or wear resistant applications for industrial processes and 
sport articles [35]. In most cases, the main reason for the use of composite materials is to improve the mechanical properties of the matrix such as strength, stiffness or toughness. Of particular interest, and a general advantage of composites, are their often high specific properties, that is, high strength and stiffness in relation to the material density. However, other properties such as the resistance to fatigue, creep, corrosion, thermal conductivity or electrical resistivity can also be modified by the incorporation of reinforcing phases [1, 35]. Ultimately, the properties of the composite are determined by the choice of reinforcement and matrix. Through the combination of different materials, the properties of the composite can be tailored to meet the specific requirements of a given application. This is a major advantage of composite materials. However, higher costs compared to conventional materials are among the disadvantages of these materials. Also, fibre composites are highly heterogeneous and generally show a marked anisotropy, i.e. the properties of the composite depend considerably on the orientation. This anisotropy has to be taken into account during the design of components.

Besides the fundamental influence of fibre and matrix, the composite properties also depend on several other key parameters including the fibre-matrix interface and its strength, the fibre content, the fibre architecture (short fibres, continuous fibres, woven fabrics), fibre orientation and the fabrication process. The fibre-matrix interface is a very critical parameter and has significant influence on the overall performance of the composite. If the interface is too weak, the load cannot be transferred onto the fibres resulting in low strength and stiffness. On the other hand, an interfacial that is too strong bond will prevent reinforcing mechanisms and cause brittle failure behaviour. The concept of fibre reinforcement of brittle matrix composites is discussed in some more detail in section 2.1.3.

\subsubsection{Materials}

A vast number of both matrix and fibre materials are currently available for the fabrication of composite materials and new or improved materials are constantly being developed. The properties and fabrication methods of the various fibres and matrix materials have been extensively studied. Comprehensive overviews on the 
subject of composite materials including common fibre reinforcements and matrix materials have been published for example by Chawla [34], Zweben [35] and Cardarelli [1]. Only some general aspects of the most commonly used fibre and matrix materials are briefly summarised here.

The fibres that are of most interest as reinforcements for composites are so-called advanced fibres. These types of fibres are generally characterised by very high strength and/or stiffness along with a low density [34]. These fibres are typically available in form of fine diameter multifilaments or, in some cases, in form of somewhat larger monofilaments. Typical diameters of fibres used in structural composites range between approximately $5-150 \mu \mathrm{m}$. Most continuous fine diameter fibres are commercially available in a number of different forms or architectures. The most basic form for fibres is in shape of multifilament tows or rovings. A fibre tow consists of a number of more or less parallel aligned fibres. The tow size can vary significantly from several hundreds to many thousands of individual fibres. These fibres can also be used to make various styles of two- and three-dimensional woven fabrics by applying similar processes to those used in textile technology.

In general, two groups of fibres can be distinguished, i.e. organic and inorganic fibres. Inorganic fibres are of greater significance for the fabrication of high performing composites and are widely used. The most common inorganic fibre types are glass, carbon, silicon carbide, boron, alumina and aluminosilicate fibres. Inorganic fibres are typically characterised by high strength, stiffness and thermal stability. The only advanced organic fibre types that currently have any considerable relevance in polymer composites are synthetically produced aramid (e.g. Kevlar ${ }^{\circledR}$ ) and to some degree high modulus polyethylene fibres [31, 34]. Organic fibres are usually cheaper than most inorganic fibres and are characterised by high strength, low density and high flexibility. However, the stiffness of organic fibres is relatively low.

As mentioned before, composite materials are often classified into three main classes according to the matrix material used. The three main groups of matrix materials are 
polymers, metals and ceramic and respective composites are called polymer matrix composites (PMCs), metal matrix composites (MMCs) and ceramic matrix composites (CMCs). There are numerous types of materials in each class of matrix material that can be used for the fabrication of composites. In the following section, only polymer and ceramics materials are considered as metal-based composites are irrelevant to the present study.

Polymers are the most widely used matrix material for composites. The strength and stiffness of polymer matrices are generally relatively low. Thus, the strength and stiffness of PMCs is strongly influenced by the properties of the reinforcing fibres. Two major classes of polymers can generally be distinguished, i.e. thermosets or thermoplastics. Both types of polymers are used for the production of composites. However, thermosetting resins play a predominant role in current PMC manufacturing [1]. Thermosets harden due to a curing reaction associated with the cross-linking of polymeric chains. The most relevant thermosetting resins for PMC manufacturing are epoxies $[1,35]$. Other thermosets that are used in composites include (unsaturated) polyester and phenolics. Thermoplastics, on the other hand, soften or melt above a certain temperature but harden when cooled to room temperature. Some common examples for this type of polymers for PMCs are polyethylene, nylon, polycarbonates and polyether etherketone (PEEK) [35].

Ceramics are typically characterised by a number of desirable properties such as high strength, stiffness and hardness, relatively low density and good chemical resistance. However, the most important property of ceramics is their superior high temperature stability. Despite all of these highly favourable characteristics, the inherent brittleness and the accompanying catastrophic failure behaviour of ceramics limits their use in structural applications. However, this limitation can be largely overcome by incorporating fibres into the brittle ceramic matrix and carefully controlling the interaction between the two components.

Two main groups of ceramic materials are often distinguished, i.e. oxide and nonoxide ceramics. The non-oxide ceramics may be further specified as carbides, 
nitrides or borides. Carbon is sometimes considered as a separate class of material due to its particular status and high relevance but may also be considered as a nonoxide ceramic. Alumina $\left(\mathrm{Al}_{2} \mathrm{O}_{3}\right)$ and aluminosilicate (e.g. mullite, $\left.3 \mathrm{Al}_{2} \mathrm{O}_{3} \cdot 2 \mathrm{SiO}_{2}\right)$ ceramics are by far the most important matrix materials for the fabrication of oxide ceramic composites. The choice of fibre reinforcement is usually limited to fibres of a similar chemical nature. Non-oxide CMCs are predominantly made using carbon and silicon carbide $(\mathrm{SiC})$ matrices and fibres. Other inorganic, non-metallic matrix materials that are of considerable interest in composite manufacture are glasses and various cements.

\subsubsection{Conventional fabrication methods}

In general, the fabrication process of a composite is dictated by two factors: (i) the type and shape of the reinforcement, and (ii) the nature of the matrix [33]. The types of fibres that are relevant to the present study are continuous fibres. Therefore, only processes involving these types of fibres are discussed here. As mentioned in the introduction of this thesis, geopolymers combine low-temperature processing similar to thermosetting resins with ceramic-like properties. Therefore, some of the general fabrication methods of thermosetting polymer-based composites are very briefly outlined below and compared to some principal aspects of ceramics manufacturing. The book "Composite Materials - Science and Engineering" by Chawla [34] and a list of fabrication techniques for PMCs by Cardarelli [1] were used as general references. For more detailed information on the manufacturing techniques of polymer and ceramic composites, the reader is referred to the specialised literature on these topics.

One of simplest and cheapest fabrication methods for PMCs is a hand lay-up. Fibres are placed into or onto a mould, and each layer is impregnated with resin using a roller. The lay-up is subsequently cured in the mould at room temperature or at slightly higher temperature in an oven. Additional pressure and/or vacuum may be applied during the curing process by using autoclaves or vacuum bagging techniques. One of the advantages of this method is the possibility to fabricate irregular shapes. 
However, the quality-control is difficult and the quality of the final product depends on the skill and experience of the manufacturer.

More advanced and automated techniques include fibre winding and pulltrusion techniques. Both methods involve the passing of fibre tows through a resin impregnation bath and the subsequent removal of excess resin before forming. In case of the fibre winding process the impregnated fibres are wound onto a mandrel. Fibres can be wound parallel or in cross patterns with various angles through adjustment of different parameters. This technique is generally able to produce large cylindrical and spherical shapes. After winding, the product has to be cured in similar ways as described for hand lay-ups. The pulltrusion technique is a continuous process that allows the fabrication of relatively large shapes with a fixed crosssection such as rods or bend and flat panels. The impregnated fibre tows are pulled through a forming and curing die of the shape of the final product.

Resin transfer moulding (RTM) is an automated method that can be used to produce complex shapes. In contrast to the techniques described before, the fibres are first made into a preform which is then placed in a closed mould and is subsequently impregnated with a low viscosity resin assisted by low pressure. The product is cured by the additional application of heat.

With ceramic matrices, processing becomes generally much more complicated and expensive. The main reasons for that are all direct or indirect consequences of the high temperatures generally required for the fabrication of ceramics. There are several techniques similar to the ones described above such as filament winding or other impregnation techniques that can be used to produce green (i.e. unfired) composite parts. All of these processes are followed by some sort of sintering or hotpressing process during which the matrix material melts or sinters, impregnates and bonds to the fibres and solidifies during cooling. The production of fibre pre-forms from continuous fibres that are subsequently infiltrated is also common for some products e.g. for SiC matrix composites. The pre-forms can be infiltrated with liquids or vapours. These techniques are called melt infiltration and chemical vapour 
infiltration (CVI) respectively. However, these processes are very complex and require special equipment and technology to control the infiltration of the pre-form. In the case of dense ceramic matrices additional costly fibre coating may have to be applied.

The main advantage of polymer matrices and PMCs, respectively, over ceramic materials and the reason why PMCs find such widespread applications is the relatively low processing temperature required for the fabrication of these materials. According to Kelly and Mortensen [33], there are several implications that evolve from that fact: first of all, the low temperatures ease the general processing considerably and allow much simpler and cheaper tooling. Secondly, the processing does not demand much energy. All these factors have direct effect on the cost and cost-efficiency. Another advantage is that a much wider range of less expensive inorganic and organic fibres can be used. The lack of problems which often arise with high temperature processing such as chemical reactivity between fibre and matrix, complex densification methods and different thermal expansion coefficients indirectly support the relative ease of PMC processing. Thus, to increase the commercial relevance and the more widespread use of CMCs, their fabrication has to become more affordable.

\subsubsection{Mechanical failure behaviour of ceramic matrix composites}

The main factor limiting the use of ceramics for structural engineering applications is their inherent brittleness and low resistance to crack propagation, i.e. low fracture toughness. One possible way to improve the toughness and damage tolerance of ceramics is by the incorporation of strong and stiff fibres. However, most of the fibres used in such composites are of an inorganic nature and are also inherently brittle. In order to achieve graceful, i.e. non-catastrophic or quasi-ductile failure of the composite, the interaction between the two components has to be carefully controlled. Composite failure is usually initiated by matrix cracks above a critical stress level. To ensure damage tolerant behaviour, it is vital that the fibres remain intact as the cracks approach the fibres. This is generally achieved if the interfacial bond strength between fibre and matrix is weak enough to allow crack deflection into 
the fibre-matrix interface and subsequent debonding of the fibre. The criterion for these debonding processes and the damage tolerant failure of the composite is described theoretically by He and Hutchinson [36] based on the critical ratio between the fracture energy of the interface $\Gamma_{\mathrm{I}}$ and the fracture energy of the fibre $\Gamma_{\mathrm{F}}$ in relation to the elastic mismatch between fibre and matrix, as shown in Figure 2.1.

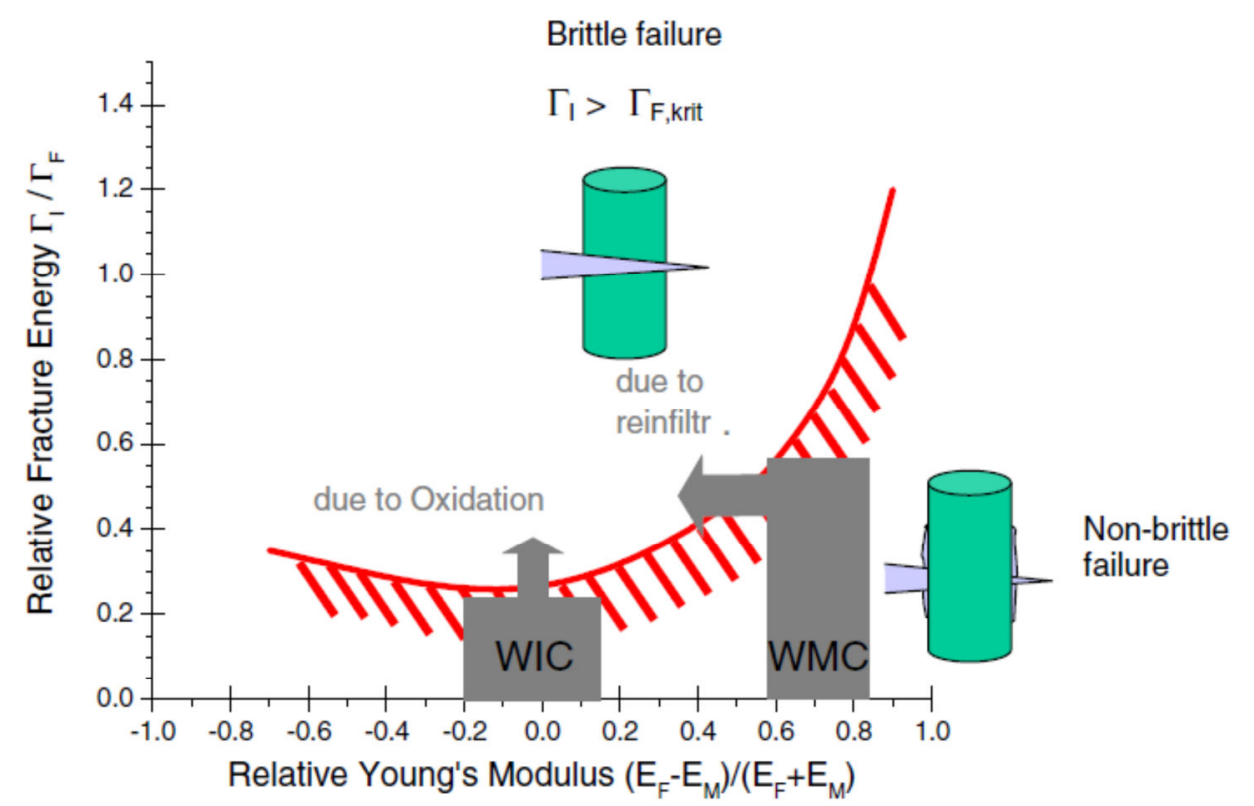

Figure 2.1: Boundary conditions of the relative fracture energy in relation to the elastic mismatch of fibre and matrix for non-brittle failure behaviour after He and Hutchinson [36], diagram from [37]

The interfacial strength can be controlled in two different ways. Both approaches aim to prevent fibre damage based on the principle of energy dissipation of stress concentrations. The first, and more conventional, approach is the application of fibre coatings. The coatings introduce a weak interface/interphase with low crack and sliding resistance to promote crack deflection and debonding between fibre and matrix. According to Koch et al., composites based on this concept may also be referred to as weak interface composites (WIC) [37, 38]. The coatings work according to two different principles: they either bond weakly, if at all, to the surface of the fibres or they are inherently weak, e.g. by being layered or porous [39]. Commonly applied fibre coatings for non-oxide ceramics such as $\mathrm{SiC}$ are carbon and boron nitride $(\mathrm{BN})$. The application of fibre coatings generally becomes a necessity with increasing density and improved mechanical properties of the matrix. This is 
evident from the He-Hutchinson diagram shown in Figure 2.1. The elastic modulus of the matrix increases with increasing density. For a highly dense matrix, similar elastic moduli for fibres $\left(\mathrm{E}_{\mathrm{F}}\right)$ and matrix $\left(\mathrm{E}_{\mathrm{M}}\right)$ may be assumed. Thus, a weaker interface is required in order to achieve fibre debonding and non-brittle failure behaviour and to fulfil the energy criterion $\Gamma_{\mathrm{I}} / \Gamma_{\mathrm{F}} \leq 0.25$. Hence, the role of the fibre coating becomes more important. If the composite is loaded above a critical stress and the energy criterion is satisfied, then several fundamental energy dissipating mechanisms can contribute to increase the fracture toughness of the composite. The principal mechanisms that operate in WIC-CMCs during crack propagation are presented in Figure 2.2 [40]. Due to the low fracture energy of the interface, stresses can be redistributed along the fibre-matrix interface without inducing fibre failure. As the main crack continues to propagate through the composite the fibres can bridge the crack surface behind the crack tip due to the higher strength and strain to failure of the fibres. This imposes a closure force on the crack that contributes to the increased toughness of the composite [41]. Continued loading causes further interfacial debonding and sliding in the wake of the crack and eventually leads to fibre fracture and pull-out.

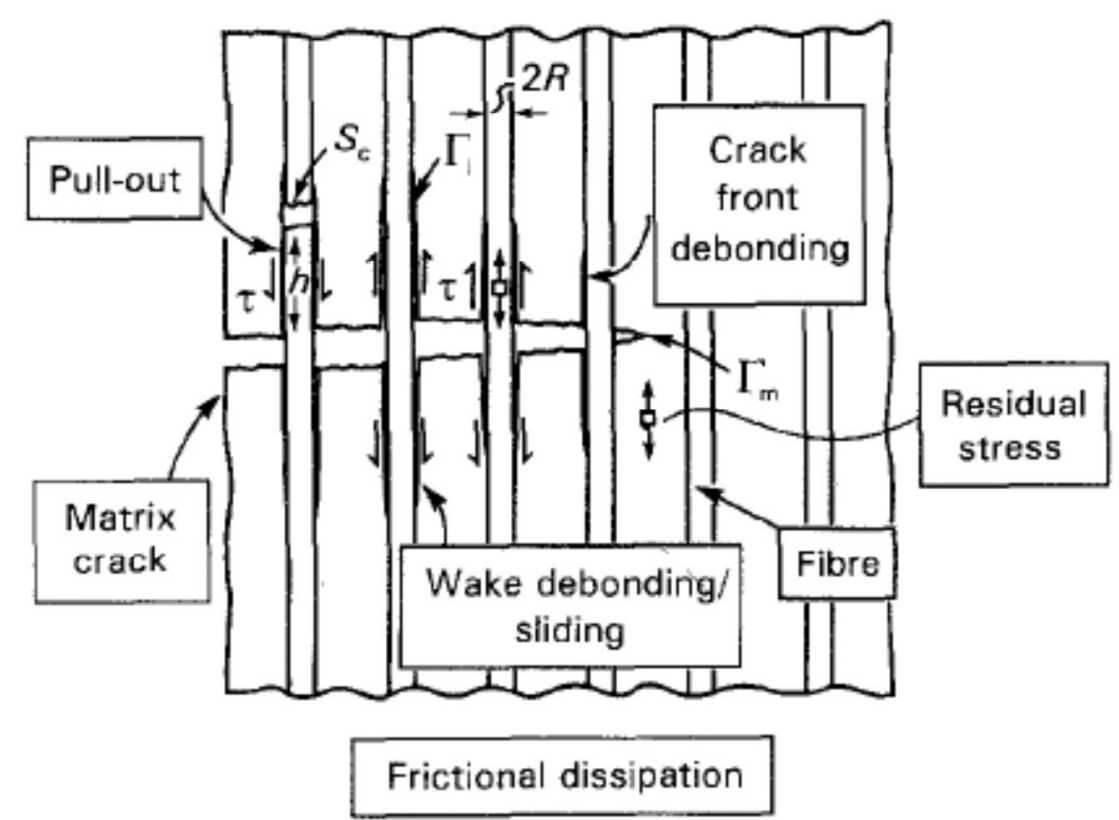

Figure 2.2: Energy dissipating mechanisms in fibre reinforced WIC-CMCs, after [40] 
The second approach to control the fibre-matrix interaction is based on the use of porous matrices. In some ways this approach can be seen as an extension to the porous coating concept [42]. However, in this case there is no need for fibre coatings to introduce a weak interfacial phase as the matrix itself acts as the weak phase [43, 44]. These types of composites are also called weak matrix composites (WMC) [37]. The concept of porous matrix composites is highly attractive as it promises a simpler and more economical composite manufacturing by eliminating the need for costly fibre coatings and elaborate matrix densification methods [45]. Porous matrix composites are of particular relevance for oxide/oxide CMCs and have been developed since the mid-1990s [39, 42, 44, 46-48]. The concept of WMCs and the reduced importance of the interfacial strength can also be displayed in Figure 2.1. Due to the dependency of the relative fracture energy $\Gamma_{\mathrm{I}} / \Gamma_{\mathrm{F}}$ on the elastic mismatch of fibre and matrix, the $\Gamma_{\mathrm{I}} / \Gamma_{\mathrm{F}}$ ratio can increase with increasing elastic mismatch without causing catastrophic failure. That is, with decreasing elastic modulus of the matrix, controlled through the matrix porosity, higher interfacial strength can be accepted. The porous matrix allows cracks to easily propagate through the matrix and deflect on matrix pores. This enables crack deflection close to the fibre interface and fibre debonding even in case of a strong fibre-matrix bond. The weak and porous matrix typically fails at low stresses under loading. However, local stress concentrations can be dissipated in the porous matrix through multiple matrix cracking. This allows the composites to carry significantly higher loads as long as load transfer onto the fibres is ensured. Because significant stress redistribution from the fibres to the matrix cannot take place due to the apparent weakness of the matrix, WMCs typically show large volume bundle failure of fibres as opposed to local fibre failure in WICs [37]. The mechanical properties and behaviour of WMCs are strongly influenced by the properties and the orientation of the fibres and the loading direction. Especially under off-axis and compression loading modes, the strength of WMCs is low [38]. 


\subsection{Geopolymers}

\subsubsection{Introduction}

The term geopolymer was originally introduced in the 1970 s by Davidovits to describe a group of newly developed amorphous to semi-crystalline aluminosilicate binder materials derived through reaction of aluminosilicate sources in aqueous alkaline media at moderately low temperatures $\left(<150^{\circ} \mathrm{C}\right)$ [49]. The initial driving force behind this research was the development of new heat resistant, non-flammable and non-combustible "plastic" materials as an alternative to common organic plastics [2]. This research eventually led to the patenting of a group of inorganic aluminosilicate binders synthesised from sodium silicate activated metakaolin [50]. Nowadays, the term geopolymer is also applied to a range of similar aluminosilicate binders synthesised from a variety of different aluminosilicate sources such as fly ash and various alkaline activation solutions, most commonly on the basis of sodium or potassium.

The main focus of geopolymer research has been and still is on the development of geopolymers as cost-efficient construction materials, in particular as a sustainable alternative to Ordinary Portland Cement (OPC). The advantages of geopolymers compared with OPC are a much lower emission of carbon dioxide during the production process, especially when using industrial waste-materials such as fly ash as the aluminosilicate source, and potentially higher durability and strength [51, 52]. However, geopolymers have also attracted considerable interest in a wide range of other applications, e.g. in the field of structural engineering, fire resistant materials and insulations as well as for the immobilisation of toxic metals [49, 53]. Nevertheless, their widespread commercial use is limited at the current stage, mostly attracting niche markets. This is largely due to the predominantly applicationoriented history of geopolymer research as indicated by the large number of patents compared to scientific publications. Also, the particular focus on traditional cement technology despite the intrinsically different nature of the chemistry of OPC and geopolymers hindered the development of a more detailed scientific understanding [54]. A more comprehensive understanding of the reaction mechanisms and the 
relationships between microstructural effects and properties of geopolymers has only been developed in recent years [54-58]. However, a complete understanding of the reaction mechanisms is essential in order to fully establish geopolymer technology and exploit the full potential of these materials.

\subsubsection{Terminologies}

In the previous section, geopolymers were introduced as a group of solid aluminosilicate material formed by reaction of an aluminosilicate source such as (meta-) kaolin and an alkali activation solution. However, there has been some controversy over the appropriate designation of this group of materials and as a result a variety of different names and terminologies can be found in the literature to essentially describe the same type of material $[59,60]$. On the other hand, the same terms are also frequently misused for a variety of other materials involving alkali activation or mineral-based processes irrespective of the fact that their structure and properties may not correspond with the defining criteria of an actual geopolymer [61]. This can leave the unfamiliar reader easily confused about the different terms and materials and supports scepticism towards geopolymer technology. Therefore, it is important to explicitly define the identifying characteristics of this group of materials. Some of the most common names and terminologies found in the literature are briefly discussed in the following paragraph.

The two most common designations for this group of materials found in the recent literature are the original term geopolymers and inorganic (aluminosilicate) polymers. The latter is sometimes considered a more appropriate broad term description [60]. However, both terms are widely accepted among researchers today. For reasons of simplicity the term geopolymer will be adopted predominantly for this study. However, both terms may be used interchangeably throughout this work. Other terms found in the literature and referring to essentially the same materials are alkali-bonded ceramics, chemically bonded ceramics, alkali-activated cement, geocement, hydroceramics, low-temperature inorganic polymer glass, (poly)sialate, and a variety of other names [3, 49, 58, 60, 62-64]. For the most part, geopolymers are defined as alkali activated aluminosilicate materials. The identifying criteria that 
distinguish geopolymers from other types of alkali activated materials are the formation of a three-dimensional, largely x-ray amorphous, aluminosilicate gel framework consisting almost exclusively of tetrahedral $\mathrm{SiO}_{4}$ and $\mathrm{AlO}_{4}{ }^{-}$units as indicated by solid state nuclear magnetic resonance (NMR) analysis [49, 61].

The polysialate terminology was introduced in the early 1980s by Davidovits in order to describe the chemical and structural nature of geopolymers [50]. The bonding between silicon and aluminium by bridging oxygen was described by a $\mathrm{Si}$ $\mathrm{O}-\mathrm{Al}$ linkage type termed sialate and a $\mathrm{Si}-\mathrm{O}-\mathrm{Si}$ linkage called a siloxo bond. The polysialate network was believed to consist of tetrahedral $\mathrm{SiO}_{4}$ and $\mathrm{AlO}_{4}{ }^{-}$units, linked by shared oxygen atoms, and alkali metal cations (e.g. $\mathrm{Na}^{+}, \mathrm{K}^{+}$). The cations, required to balance the negative charge of $\mathrm{Al}^{3+}$ in IV-fold coordination, were thought to be located in the framework cavities. A strict formalism was developed by Davidovits to envisage different structures as fundamental structural building units of the poly-condensed framework. The following empirical formula was proposed for polysialates [49]:

$$
\mathrm{M}_{\mathrm{n}}\left[-\left(\mathrm{SiO}_{2}\right)_{\mathrm{z}}-\mathrm{AlO}_{2}\right]_{\mathrm{n}} \cdot \mathrm{w} \mathrm{H} \mathrm{H}_{2} \mathrm{O}
$$

where $\mathrm{M}$ is an alkali cation (e.g. $\mathrm{Na}^{+}, \mathrm{K}^{+}$), $n$ is the degree of polymerization and $z$ can attain a value of 1,2 or 3. Hence, three main sialate building units based on the presence of only $\mathrm{Si}-\mathrm{O}-\mathrm{Si}$ and $\mathrm{Si}-\mathrm{O}-\mathrm{Al}$ linkages were distinguished in order to describe the composition of geopolymers in terms of their Si/Al ratio, see Table 2.1. These structural units or oligomers were thought to condense randomly into crosslinked chains or ring polymers to build up the x-ray amorphous framework [49, 62]. 
Table 2.1: Formal representation of the three fundamental sialate units

\begin{tabular}{|c|c|c|c|}
\hline Fundamental unit & Composition & $\mathrm{Si} / \mathrm{Al}$ & Abbreviation \\
\hline Sialate & $\underset{\mid}{\mid} \underset{\mid}{-S i-O}-\stackrel{\mid}{\mid} \mid$ & 1 & PS \\
\hline Sialate-siloxo & $\begin{array}{c}\mid \\
\mid-S i-O \\
\mid\end{array}$ & 2 & PSS \\
\hline Sialate-disiloxo & 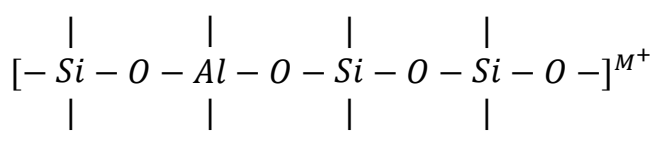 & 3 & PSDS \\
\hline
\end{tabular}

This initial model of distinct fundamental sialate units along with Davidovits' early work on geopolymers [49, 50, 65-67] formed the basis of the general understanding of geopolymers for many years and is therefore frequently referenced in publications prior to about the mid-2000s when introducing geopolymers. However, the practical use of this nomenclature is very limited and with an increasing scientific understanding of geopolymers seems to slowly disappearing from more recent publications. The simplicity of the polysialate model in providing a basic nomenclature and a graphic illustration of key building units forming the complex amorphous geopolymer structure is probably its main advantage. However, several aspects do not correspond to reality. For example, the strict formalism of the original model with only three structural sialate units proves inadequate to describe the possible composition range of geopolymers. Compositions with higher and noninteger $\mathrm{Si} / \mathrm{Al}$ ratios are not considered despite the fact that the $\mathrm{Si} / \mathrm{Al}$ ratios of many compositions in practice show non-integer values and can be well in excess of $\mathrm{Si} / \mathrm{Al}$ $=3$ [68]. Nevertheless, the polysialate terminology was applied in many of the early publications on geopolymers often without providing further detail on the actual composition of the material. Thus, it is often confusing and difficult to compare the results obtained in those studies with other works. Another limitation of this nomenclature is that it provides a one-dimensional description of a three-dimensional network [69]. Furthermore, the description of the polysialates as ring and chain polymers is not verified by experimental studies [54]. So is the assumption that 
polysialate units only comprise $\mathrm{Si}-\mathrm{O}-\mathrm{Si}$ and $\mathrm{Si}-\mathrm{O}-\mathrm{Al}$ linkages, excluding Al-O-Al linkages due to an interpretation of the Loewenstein Rule [70]. Loewenstein's Rule states that two tetrahedral coordinated aluminium atoms in a three-dimensional framework cannot be linked by one oxygen atom. Although the aluminium avoidance is often assumed to be strictly obeyed in tetrahedral aluminosilicate structures, it is not strictly forbidden, but rather a thermodynamic preference towards the avoidance of Al-O-Al bonds [54]. Since Al-O-Al linkages are not strictly forbidden, they may be present to some degree in geopolymers and violate against the total exclusion in the Davidovits model. The model is also not able to predict the effect of variations of the $\mathrm{Si} / \mathrm{Al}$ ratio or the type of alkali cation on the structure and the correlated properties of the material.

\subsubsection{Materials and synthesis}

The synthesis of geopolymers is relatively simple and generally requires two sources: (i) a reactive solid component containing sufficient amounts of reactive silica and alumina species and (ii) an alkali activation solution that contains alkali hydroxides, silicates and sometimes aluminates or combinations thereof [63]. That generally offers a wide variety of raw materials that can be used to produce geopolymers. One of the most common geopolymer synthesis methods is the original method of Davidovits involving the reaction of a 1:1 layer-lattice aluminosilicate clay mineral such as kaolin with an alkali silicate solution under highly alkaline conditions [49]. In most cases, the clay mineral is thermally activated prior to its use to increase its reactivity. The calcined form of kaolin is generally known as metakaolin. It has been shown that the calcination of kaolin has a significant effect on the characteristics of the geopolymer. Geopolymers formed from metakaolin show a higher reactivity and yield higher early strength, while geopolymers formed from untreated kaolin often result in weak, poorly reacted products. The degree of dehydroxylation or calcination of kaolin has been studied previously [71]. In the metakaolin region between $550^{\circ} \mathrm{C}$ to about $900^{\circ} \mathrm{C}$, the degree of dehydroxylation is nearly constant and the required calcination times decreases with increasing temperature. However, sinter effects can occur at higher temperatures and long annealing times, lowering the reactivity of the material. Other common aluminosilicate sources for the synthesis of geopolymers are 
fly ash, blast furnace slag, various clays, naturally occurring minerals and various combinations thereof $[50,65,66,72-77]$. At the current stage, fly ash geopolymers take up the majority of application-oriented research. Metakaolin geopolymers, on the other hand, are mostly studied for scientific purposes due to their higher purity and homogeneity compared to fly ash geopolymers, making it easier to characterise these materials. However, it is unclear to what degree the information obtained from the 'model' metakaolin geopolymers can be transferred to fly ash geopolymers [78]. Nevertheless, this study will mainly focus on metakaolin geopolymers.

The variety of possible raw materials offers a great advantage in terms of accessibility, functionality and cost-effectiveness of geopolymer materials. On the other hand, this also proves to be a great disadvantage at the same time as there is little restriction on the purity, particle size, composition or morphology of the materials that can be used [54]. All these factors have considerable impact on the general processing characteristics, the microstructure and the overall performance of the final product. Since the raw materials that are used in the various studies on geopolymers are often of local nature with quite variable characteristics, it is very difficult to compare the properties of geopolymers derived from different systems. Even if the theoretical composition of two geopolymers derived from different raw materials is the same, properties and particularly reaction kinetics can be completely different due to impurities and varying reactivity of the raw materials. Also, there is no consensus on the general processing parameters which can also have considerable effect on the resulting properties of the material. Besides all these factors, the microstructure and properties such as strength and durability also depend on the general composition of the geopolymer mixture. Although there is no designated 'standard composition' and viable materials can be formed from a much wider range of compositions, there seems to be some agreement on what is considered to be an optimal composition range for the production of strong and hard geopolymers: $\mathrm{SiO}_{2} / \mathrm{Al}_{2} \mathrm{O}_{3} \sim 3-4, \mathrm{M}_{2} \mathrm{O} / \mathrm{SiO}_{2} \sim 0.25-0.4$ and $\mathrm{H}_{2} \mathrm{O} / \mathrm{M}_{2} \mathrm{O} \sim 10-11 ; \mathrm{M}: \mathrm{Na}^{+}$or $\mathrm{K}^{+}[23$, 90]. Particularly for the fabrication of geopolymer composites a rather low viscosity of the geopolymer binder is generally favoured in order to improve the impregnation of fibre bundles. However, the viscosity cannot simply be lowered by adding extra 
water to the geopolymer binder as the strength of the hardened geopolymer decreases significantly with increasing water content. Therefore, not every geopolymer composition is suitable for use as a composite matrix material. The molar ratio itself, however, does not give any indication of the viscosity of the binder. The mechanical properties of the geopolymer matrix also depend on the nature of the alkali cations present in the structure to balance the negative charge associated with the tetrahedral $\mathrm{AlO}_{4}{ }^{-}$units. These charge-balancing cations are typically alkali metal ions, most commonly $\mathrm{Na}^{+}$because of its comparative cheapness, and $\mathrm{K}^{+}$. Although potassium geopolymers have been found to obtain somewhat higher strength than sodium geopolymers [79], the $\mathrm{M}_{2} \mathrm{O} / \mathrm{SiO}_{2}$ ratio seems to be a much more critical factor for the synthesis and performance of geopolymers [58].

The synthesis of geopolymers essentially involves a mixing process of some sort to form a homogeneous binder. Vacuum, vibration or other additional procedures may also be applied in order to minimize the amount of entrapped air in the liquid binder. However, there is no consensus on conforming processing parameters despite a considerable influence of the processing parameters on the material properties. For example, it is not hard to imagine that a geopolymer made from a composition mixed mechanically under vacuum will possess a more homogeneous microstructure and higher strength than the exact same mix stirred by hand. This is another major factor that limits the comparability of different geopolymer compositions. The subsequent curing process is one of the most crucial steps in the synthesis of geopolymers and has a significant effect on the properties of the final product. The curing time and temperature are the key variables in this process. Curing temperatures of 40 to $80^{\circ} \mathrm{C}$ are most common but may range from room temperature up to $>100^{\circ} \mathrm{C}$. Curing times vary from several hours up to 28 days, the time used as a standard for concrete [51]. However, curing times of 24 to 48 hours are most common for geopolymers. In order to prevent unfavoured excessive water evaporation during the setting and curing of the geopolymer binder which can lead to lower strength and the formation of drying cracks, specimens have to be sealed or cured under controlled humidity. Other synthesis methods, including a sol-gel method [80] and a solid-state method [81] have also been developed but are beyond the scope of this thesis. 


\subsubsection{Geopolymerisation process}

As described before, geopolymers form by reaction of aluminosilicate materials with highly alkaline activation solutions to produce viscous binders that subsequently harden at room or slightly elevated temperatures to form rigid solids [49]. The reactions that take place during the geopolymer formation and solidification processes are generally described by the overall term 'geopolymerisation'. However, the geopolymerisation process is complex and currently not completely understood. Several mechanisms have been proposed in the literature with most of them agreeing on four basic steps that occur during geopolymerisation: (i) dissolution of silica and alumina in highly alkaline solution, (ii) diffusion or transportation of the dissolved species, (iii) poly-condensation and gel-formation and (iv) hardening of the gel phase that results in the final geopolymer [59, 67, 73, 82-84]. However, these steps are not distinct processes that can be investigated separately but overlap and occur more or less simultaneously [85]. The geopolymerisation process continues after the hardening of the geopolymer structure and studies by White et al. [86] suggest that structural changes of geopolymer gel phase, described as the transition from a gell to a gel2 state, occur over the course of 90 days. Geopolymerisation is an exothermic process [67], and is affected by the nature and pre-treatment of the aluminosilicate source and the type and concentration of the alkali activation solution $[83,85]$. Other researchers have also suggested models based on the understanding of the formation of zeolites [87]. These authors proposed that the geopolymeric binder phase consists of an agglomeration of zeolitic nano-crystallites bound together by an aluminosilicate gel. However, transmission electron microscope (TEM) investigations of a geopolymer composition by Schmücker et al. [88] did not confirm the existence of any nanocrystalline structures.

\subsubsection{Chemical and structural aspect of metakaolin geopolymers}

Metakaolin geopolymers are often considered as 'model systems' for basic scientific investigations due to the relative simplicity of the materials system. However, it has to be noted that no specific model system exists and the quality of the wide range of metakaolin sources used for the production of geopolymers can vary considerably in purity, crystallinity, particle size and general reactivity depending on, for example, 
the dehydroxylation parameters. All these factors have significant effect on the fabrication and properties of geopolymers. Other factors are of course the $\mathrm{Si} / \mathrm{Al}$ ratio of the geopolymer, the water content and the type, amount and concentration of the alkali activation solution, as mentioned before. Therefore, some considerable variation between different studies has to be expected. In the following section, some general aspects of the chemistry and nano-/microstructure of metakaolin geopolymers are briefly discussed.

Solid state magic angle spinning nuclear magnetic resonance spectroscopy (MASNMR) is a valuable analytical technique for the structural characterisation of geopolymers and has been utilized in numerous studies to analyse geopolymer formation and the structural changes upon heating [49, 64, 77, 89]. Evidence for the presence of tetrahedral silicate and aluminate units in metakaolin geopolymers has been provided for example by Barbosa et al. [90]. NMR studies by Rowles et al. have also provided information on the location and hydration states of the monovalent charge-balancing cations [91]. Based on those studies, several structural models have been proposed for the nanostructure of the geopolymer framework. The most recent and advanced model is the one presented by Rowles et al. [91]. According to these authors, the sodium cations are not only present in a solely hydrated form as suggested in the earlier model of Barbosa et al. [90], but in a range of hydrated states and also sometimes show short range order (Figure 2.3a, b). The revised schematic model of the framework structure of metakaolin geopolymer proposed by Rowles et al. [91] is presented in Figure 2.3c. However, it has to be noted that this two-dimensional model can only provide an indicative view of the complex three-dimensional geopolymer framework. 


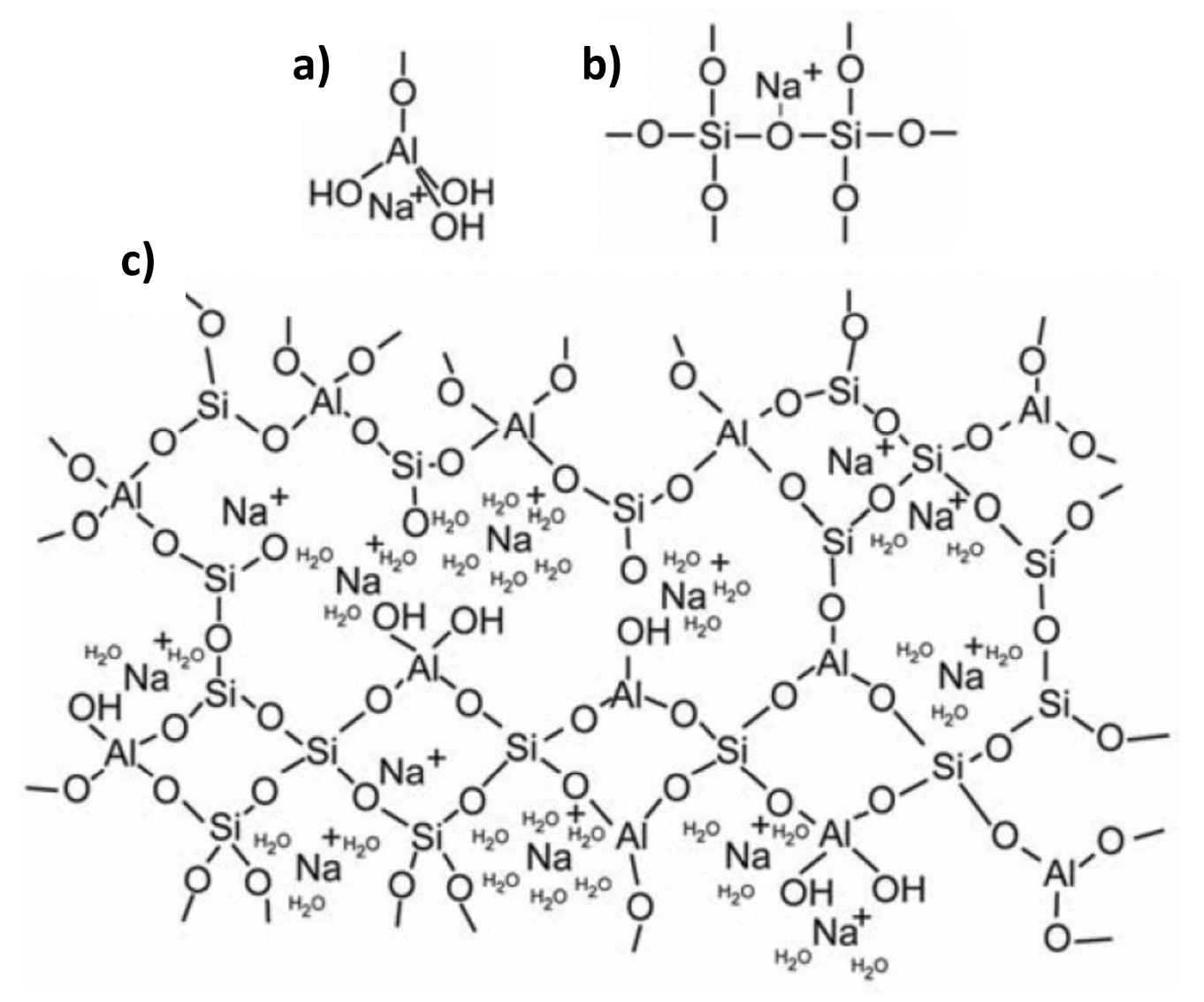

Figure 2.3: Proposed structural model for metakaolin geopolymers by Rowles et al. [91]

The structure of geopolymers is broadly described as x-ray amorphous. For well cured metakaolin geopolymers, the major feature of standard powder x-ray diffraction (XRD) patterns is a featureless hump typically around approximately 27$29^{\circ} 2 \theta(\mathrm{Cu} \mathrm{K} \alpha)$. Their $\mathrm{x}$-ray amorphous character has been reported to remain largely unchanged on heating $>1000^{\circ} \mathrm{C}$ [89]. However, crystallisation can occur at much lower temperatures, depending on several factors including the overall composition, water content and curing conditions. Some crystalline phases such as quartz can sometimes be found in the diffraction pattern of cured geopolymers and are a result of unreactive impurities in the metakaolin. XRD is usually utilized to prove the general amorphousness of the material and to document phase crystallisation upon heating. 


\subsubsection{Properties}

When introducing geopolymer materials, their properties are often described in very general, well-sounding terms such as temperature stable and fire proof, corrosion resistant, rapid hardening at low temperatures, high (early) strength and so on. These terms are obviously of no great value with regard to characterising the properties of a material. However, it is somewhat difficult to be more specific when talking about geopolymers in general since the properties vary over such a wide range that it is nearly impossible to name some "typical" properties without having to elaborate on further details of the material system, the composition, etc. Many of the factors that affect the performance of geopolymers have already been mentioned several times throughout this chapter. Besides the choice of raw material system, overall compositional ratios, processing and curing parameters, the different testing methods and parameters are also important factors that reflect in the measured properties. Due to the fact that no standardised testing parameters for geopolymers have been established, various different testing standards and parameters are applied for the characterisation of geopolymers. Since one of the principal applications envisaged for geopolymers is in the construction industry as an alternative for Portland cement, concrete testing standards have been widely adapted for geopolymers. However, other standards, generally used for the testing of polymer and ceramic specimens, respectively, have also been employed. These latter standards usually work on the basis of much smaller specimens. Therefore, the comparability between results of different studies is often very limited. Also, the main mechanical characterisation method for concrete is compressive strength measurements. Thus, most studies of the mechanical properties of geopolymers are often limited to only this means of characterisation. This may be sufficient for geopolymer concretes and can be used as a relatively simple and cost-efficient evaluation test but a more detailed characterisation of the engineering properties is essential for more advanced applications of geopolymers such as in structural composite materials.

The compressive strengths of geopolymer materials span a wide range from essentially close to zero up to around $100 \mathrm{MPa}$ [58]. Brooks et al. reported one of the highest compressive strength of $110 \mathrm{MPa}$ for a fly ash geopolymer activated with a 
sodium silicate and hydroxide solution [92]. However, compressive strength values in the range of $30-70 \mathrm{MPa}$ are most commonly reported. The mechanical properties of geopolymers are strongly dependent on their chemical composition. The influence of the $\mathrm{Si} / \mathrm{Al}$ ratio on the compressive strength of metakaolin geopolymers was investigated for example by Duxson et al. [56]. These authors found that the strength increases with increasing $\mathrm{Si} / \mathrm{Al}$ ratios from 1.15 to 1.9 , achieving a maximum strength of around $75 \mathrm{MPa}$. Higher $\mathrm{Si} / \mathrm{Al}$ ratios, however, resulted in a lower strength [56]. The effects of $\mathrm{NaOH}$ concentration and composition of the alkali activation solution on the mechanical properties of sodium activated metakaolin geopolymers have been investigated by Wang et al. and Granizo et al. [93, 94]. An increase of compression and flexural strength was observed for increasing $\mathrm{NaOH}$ concentration from 4-12 mol/L [93]. Studies by Granizo et al. suggest that higher compressive strength can be obtained with activation solutions consisting of a mixture of sodium silicate and $\mathrm{NaOH}$ compared to samples activated with solutions based solely on $\mathrm{NaOH}$ [94]. It should also be noted that the strength of geopolymers is usually subject to changes over time. Metakaolin geopolymers generally show high early strength whereas geopolymers based on fly ash and ground granulated blast furnace slag (GGBFS) often develop a higher strength over a longer period of time. The effects of time and accelerated aging on the performance of metakaolin and fly ash geopolymers are discussed by Lloyd [95].

Although compressive strength values are available for many geopolymer matrix materials, data are much sparser in the case of their flexural strengths and elastic properties, which are of greater interest in the context of fibre reinforced composites. A selection of the available mechanical properties of some metakaolin geopolymers which have been used as matrices for fibre reinforced composites is presented in Table 2.2. The significant variation of the flexural strength for basically identical nominal matrix compositions as well as the partially incomplete property data should be noted. Mechanical properties of a commercially available geopolymer (Geopolimite $^{\mathrm{TM}}$, in the early 1990s) used as a matrix material for fibre composites have been reported by Foerster [3]. Besides the flexural strength and elastic modulus 
of around $15 \mathrm{MPa}$ and $10 \mathrm{GPa}$, respectively, this author also reports a very low fracture toughness $\mathrm{K}_{1 \mathrm{c}}$ of $0.29 \mathrm{MPam}^{0.5}$.

Table 2.2: Reported mechanical properties and compositions of metakaolin geopolymers used as composite matrices

\begin{tabular}{lcccc}
\hline & {$[9]$} & {$[23-25]$} & {$[20]$} & {$[13]$} \\
\hline Composition & & & & \\
Alkali Ion $\left(\mathrm{M}^{+}\right)$ & $\mathrm{K}^{+}$ & $\mathrm{K}^{+}$ & $\mathrm{K}^{+}$ & $\mathrm{K}^{+}$ \\
$\mathrm{SiO}_{2} / \mathrm{Al}_{2} \mathrm{O}_{3}$ & $\mathrm{n} / \mathrm{a}$ & 4 & 4 & 3 \\
$\mathrm{M}_{2} \mathrm{O} / \mathrm{SiO}_{2}$ & $\mathrm{n} / \mathrm{a}$ & 0.3 & 0.3 & 0.33 \\
$\mathrm{H}_{2} \mathrm{O} / \mathrm{M}_{2} \mathrm{O}$ & $\mathrm{n} / \mathrm{a}$ & 11 & 11 & 11 \\
Tension & & & & \\
Strength $\sigma_{\mathrm{t}}[\mathrm{MPa}]$ & 3.7 & $\mathrm{n} / \mathrm{a}$ & $\mathrm{n} / \mathrm{a}$ & $\mathrm{n} / \mathrm{a}$ \\
Compression & & & & \\
Strength $\sigma_{\mathrm{c}}[\mathrm{MPa}]$ & 39 & 45 & $\mathrm{n} / \mathrm{a}$ & $\mathrm{n} / \mathrm{a}$ \\
Flexure & & & & \\
Strength $\sigma_{\mathrm{f}}[\mathrm{MPa}]$ & 8.1 & 1.7 & 16.8 & 12.3 \\
Modulus $\mathrm{E}_{\mathrm{f}}[\mathrm{GPa}]$ & 9.4 & $\mathrm{n} / \mathrm{a}$ & $\mathrm{n} / \mathrm{a}$ & 10.3 \\
Strain $\varepsilon_{\mathrm{f}}[\%]$ & 0.09 & $\mathrm{n} / \mathrm{a}$ & $\mathrm{n} / \mathrm{a}$ & $\mathrm{n} / \mathrm{a}$ \\
\hline
\end{tabular}

In regards to their thermal behaviour, geopolymers are often simply described as being temperature stable and fire proof. Although geopolymers can indeed resist fire without burning due to their inorganic nature and largely retain their microstructural features, i.e. $\mathrm{x}$-ray amorphousness and tetrahedral coordination of $\mathrm{Al}$, heat treatment of solid geopolymer bodies can result in substantial shrinkage and cracking. This is due to the release of water and the onset of sintering effects, melting and crystallisation. Metakaolin geopolymers particularly can show early drying cracks even at low temperatures. The melting point and crystallization products depend on the chemical composition of the geopolymer, the selected raw materials and the type of alkali used. According to the phase diagram for the system $\mathrm{SiO}_{2}-\mathrm{Al}_{2} \mathrm{O}_{3}-\mathrm{Na}_{2} \mathrm{O}$, sodium geopolymers within this system often crystallise to nepheline $\left(\mathrm{NaAlSiO}_{4}\right)$ at about $1100^{\circ} \mathrm{C}$. This was generally confirmed by studies of Barbosa et al. although the exact crystallization temperature and phase formation depends on the exact composition [89]. Potassium geopolymers have been reported to form crystalline 
kalsilite $\left(\mathrm{KAlSiO}_{4}\right)$ and/or leucite $\left(\mathrm{KAlSi}_{2} \mathrm{O}_{6}\right)$ at temperatures above $1100^{\circ} \mathrm{C}[13$, 96]. Higher thermal stability has been observed for Cs-activated geopolymers [97].

\subsection{Geopolymer matrix composites}

Fibre reinforced geopolymer matrix composites were first investigated in the 1980s by Davidovits et al. with the objective to fabricate moulding tools and patterns for the plastic processing industry $[49,98,99]$. The developments of the early research on geopolymer composites were summarised by [2]. However, the scientific relevance of this work is somewhat limited and the reader is referred to the literature for further information about these works. Subsequent studies of the mechanical and thermal properties of fibre reinforced geopolymer composites were carried out by Foerster [3-5], Lyon et al. [6, 7], Foden [8,9] and Hammel [10-12] throughout the 1990s. For the most part, these works form the basis of geopolymer composite research at the current stage. However, the work by Foerster has gone largely unrecognised probably due to the use of an alternative terminology to describe the de facto geopolymer matrix (this problem was discussed in chapter 2.2.2) as well as the fact that several important aspects of his work were not published in English language. More recent work on continuous fibre reinforced geopolymer composites has been presented by He [13-15]. Several other publications on the topic are available, most of which focus on short fibre reinforced composites [20-30]. However, short fibre composites are beyond the scope of this study. In the following sections, the state of the art of continuous fibre reinforced geopolymers matrix composites is summarised and discussed in some more detail.

Fibre reinforced geopolymer composites have been studied almost exclusively using metakaolin-based matrices, most commonly in combination with potassium alkali activation solutions. Some properties of selected matrices have already been presented in Table 2.2. The majority of studies were carried out using carbon fibre reinforcements. However, the use of a variety of other inorganic fibre types such as glass, silicon carbide and several types of aluminosilicate fibres has also been 
reported. Basalt is another type of fibre that has been used to some extent for geopolymer composites, however, mostly in form of short fibre reinforcement [16, 23-28]. The only use of continuous organic fibre reinforcements, in this case unidirectional aramid fibres, was reported by Foerster [3].

The nature of geopolymers to form solid ceramic-like materials at near-ambient temperatures from a viscous aluminosilicate binder presents new possibilities for the fabrication of ceramic composites. The similarity between the setting behaviour of geopolymer binders and thermosetting polymers generally allows the adoption of processing techniques frequently applied in the fabrication of PMCs. These processing methods have so far been unavailable for CMCs. Some of the advantages of the near room temperature processing have already been mentioned in chapter 2.1.2. These advantages generally transfer to the fabrication of geopolymer composites. Thus, geopolymer composites have a great potential to produce costefficient CMC-like materials with PMC-like processing methods. Hand impregnation and subsequent lay-up of unidirectional and woven fibre layers is the most common method that has been used in the past to fabricate geopolymer composites. However, there are several other methods that have been applied. The possibility of prepregging and storage of geopolymer-impregnated fibre layers was investigated by Hammell who found that samples could be stored for up to 28 days at $-15^{\circ} \mathrm{C}$ without any loss of strength [11]. However, the elastic modulus was found to be unfavourably affected. The general applicability of a pulltrusion/filament winding process was demonstrated by [16-18] although these authors only used this process to impregnate continuous fibre tows. The final composite was also produced by hand lay-up of cut impregnated fibre tows. The successful preparation of fibre-reinforced engine exhaust valves by a resin transfer moulding process using a geopolymer binder has been reported by Radford et al. [19]. Similar to unreinforced geopolymers, the final curing process also affects the properties of the composite. In general, geopolymer composites can be cured similarly to their unreinforced counterparts, i.e. under controlled temperature and humidity. However, pressure and vacuum-assisted methods may also be used to improve the impregnation of fibre bundles, reduce pore volume, assist the densification of the matrix and to press out excess binder, thereby 
increasing the fibre volume content in the composite. In this regard, vacuum bagging is a commonly used process for the fabrication of geopolymer composites. Combinations of pressure and heat-assisted vacuum bag curing have been employed by $[7,9,17,21]$. Typical fibre contents are in the range of $20-50 \mathrm{vol} \%$ although fibre contents as high as $70 \mathrm{vol} \%$ have been claimed by Foerster for unidirectional carbon and aramid fibre reinforced composites [3].

\subsubsection{Properties}

The most common method described in the literature used to characterise the mechanical properties of geopolymer composites is the flexural strength test. In most cases three-point bending tests are used but four-point test setups have also been applied. The previous chapter showed that the properties of geopolymer matrices can vary significantly depending on the choice of raw materials, matrix composition and processing parameters among other factors. The same is true of their corresponding composites. However, in addition to these factors, comparison of the properties of continuous fibre reinforced geopolymer composites is complicated by the nonstandardized testing parameters for these materials. The testing parameters such as the dimensions of test specimens as well as the corresponding span-to-depth ratio, i.e. the ratio of testing span to sample thickness, applied in the individual studies vary in a wide range. These parameters can have significant effect on the measured strength and the failure behaviour of the material. As an example, the approximate dimensions and span-to-depth ratios of unidirectional fibre reinforced test specimens applied in four selected studies are given: $4 \times 3 \times 36 \mathrm{~mm}(10: 1)$ [13], 5x5x60 mm (10:1)

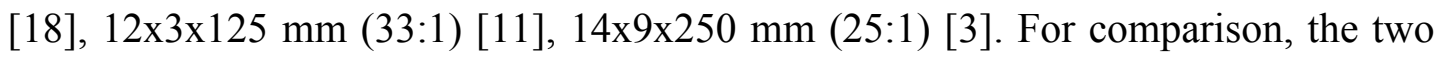
ASTM testing standards that may be most applicable as guidelines for the testing of geopolymer composites, i.e. ASTM D-790 and ASTM C-1341 which are the common standards for flexural strength testing of continuous fibre reinforced PMCs and CMCs, respectively, both suggest a minimum span-to-depth ratio of 16:1 to limit shear stresses in the material during testing. Higher ratios of 32:1 or even $64: 1$ are suggested for composites with weak shear properties in order to obtain tensile rather than shear failure during flexural testing. Although not widely investigated, a relatively low shear strength for geopolymer composites can be assumed due to the 
fine porosity and low strength and stiffness of geopolymer matrices. Thus, span-todepth ratios of $>32: 1$ would appear more sensible. Yet, two of the selected exemplary studies use a span-to-depth ratio of about 10:1 and it is not surprising that both authors report a combination of shear and compression failure [13, 18]. However, similar failure behaviour has also been reported by Foden for carbon fibre composites at higher span-to-depth ratios [9]. This leaves the question if the reported strengths can actually be considered as a flexural strength and what parameters should be applied for the testing of geopolymer composites. Therefore, the obtained results have to be considered carefully.

The flexural properties of selected geopolymer composites reported in the literature are presented in Table 2.3 and Table 2.4 distinguished into unidirectional (UD) and fibre fabric reinforced composites. Fabric reinforcements, particularly carbon fabrics in various forms, are the most widely used type of fibre reinforcement in geopolymer composites. The highest flexural strength of $310 \mathrm{MPa}$ was reported by Hammell with a 1 k, i.e. 1000 filaments per tow, carbon fabric [11]. As can be seen in Table 2.3 a clear trend of decreasing strength and elastic modulus with increasing fabric tow size can be observed. This was explained by Hammel in terms of the greater fibre undulation in the fibre weave at larger tow sizes and the corresponding lower

Table 2.3: Maximum flexural properties of selected fibre fabric reinforced geopolymer composites at room temperature

\begin{tabular}{|c|c|c|c|c|}
\hline Fibre type & $\begin{array}{c}\text { Fibre } \\
\text { content }\end{array}$ & $\begin{array}{c}\sigma_{f, \max } \\
(\mathrm{MPa})\end{array}$ & $\begin{array}{l}\mathbf{E}_{\mathbf{f}, \max } \\
(\mathbf{G P a})\end{array}$ & Reference \\
\hline Carbon & 32 vol $\%$ & 54 & 21 & [3] \\
\hline $\mathrm{Al}_{2} \mathrm{O}_{3} / \mathrm{SiO}_{2}$ & 27 vol\% & 86 & 29 & 6 \\
\hline $\mathrm{Al}_{2} \mathrm{O}_{3} / \mathrm{SiO}_{2} / \mathrm{B}_{2} \mathrm{O}_{3}$ & 30 vol $\%$ & 66 & 18 & “6 \\
\hline Carbon & $60 \mathrm{vol} \%$ & 65 & 18 & “" \\
\hline $\mathrm{Al}_{2} \mathrm{O}_{3} / \mathrm{SiO}_{2}$ & $60 \mathrm{vol} \%$ & 142 & 36 & “6 \\
\hline Carbon 3k & $\mathrm{n} / \mathrm{a}$ & 245 & 45 & [9] \\
\hline $\mathrm{SiC}$ & $\mathrm{n} / \mathrm{a}$ & 202 & 57 & “" \\
\hline Carbon $1 \mathrm{k}$ & $\mathrm{n} / \mathrm{a}$ & 310 & 82 & [11] \\
\hline Carbon $3 \mathrm{k}$ & $\mathrm{n} / \mathrm{a}$ & 245 & 66 & 66 \\
\hline Carbon $12 \mathrm{k}$ & $\mathrm{n} / \mathrm{a}$ & 140 & 42 & “6 \\
\hline Carbon 50k & $\mathrm{n} / \mathrm{a}$ & 85 & 20 & “6 \\
\hline E-glass & $\mathrm{n} / \mathrm{a}$ & 118 & 43 & “6 \\
\hline Carbon $3 \mathrm{k}+$ E-glass & $\mathrm{n} / \mathrm{a}$ & 250 & 55 & “6 \\
\hline $\mathrm{Al}_{2} \mathrm{O}_{3} / \mathrm{SiO}_{2} / \mathrm{B}_{2} \mathrm{O}_{3}$ & 47 vol $\%$ & 97 & 36 & [19] \\
\hline
\end{tabular}


resistance of fibres to withstand fibre buckling under compressive load [11]. Fibre buckling of the extreme compression fibre was found to be the main factor in initiating composite failure. Similar compression failure with corresponding fibre buckling and delamination was reported by Foden [9]. The corresponding loaddisplacement curves show that the load typically drops rapidly after failure initiation but can sustain a constant residual strength of roughly $1 / 3$ of the ultimate flexural strength [9]. However, this observation does not correspond with the stress-strain behaviour of carbon fabric composites reported by Foerster, Figure $2.4[3,5]$. Foden also compared the stress-strain behaviour of 3 and $6 \mathrm{~mm}$ thick carbon fibre fabric reinforced specimens [9]. The general trend of the stress-strain behaviour as described above was found to remain largely unchanged upon heating to $800{ }^{\circ} \mathrm{C}$ in air $6 \mathrm{~mm}$ thick samples. For $3 \mathrm{~mm}$ thick samples a similar initial linear elastic region was observed with increased flattening of the stress-strain curve around the maximum stress. This effect became more obvious with exposure to higher

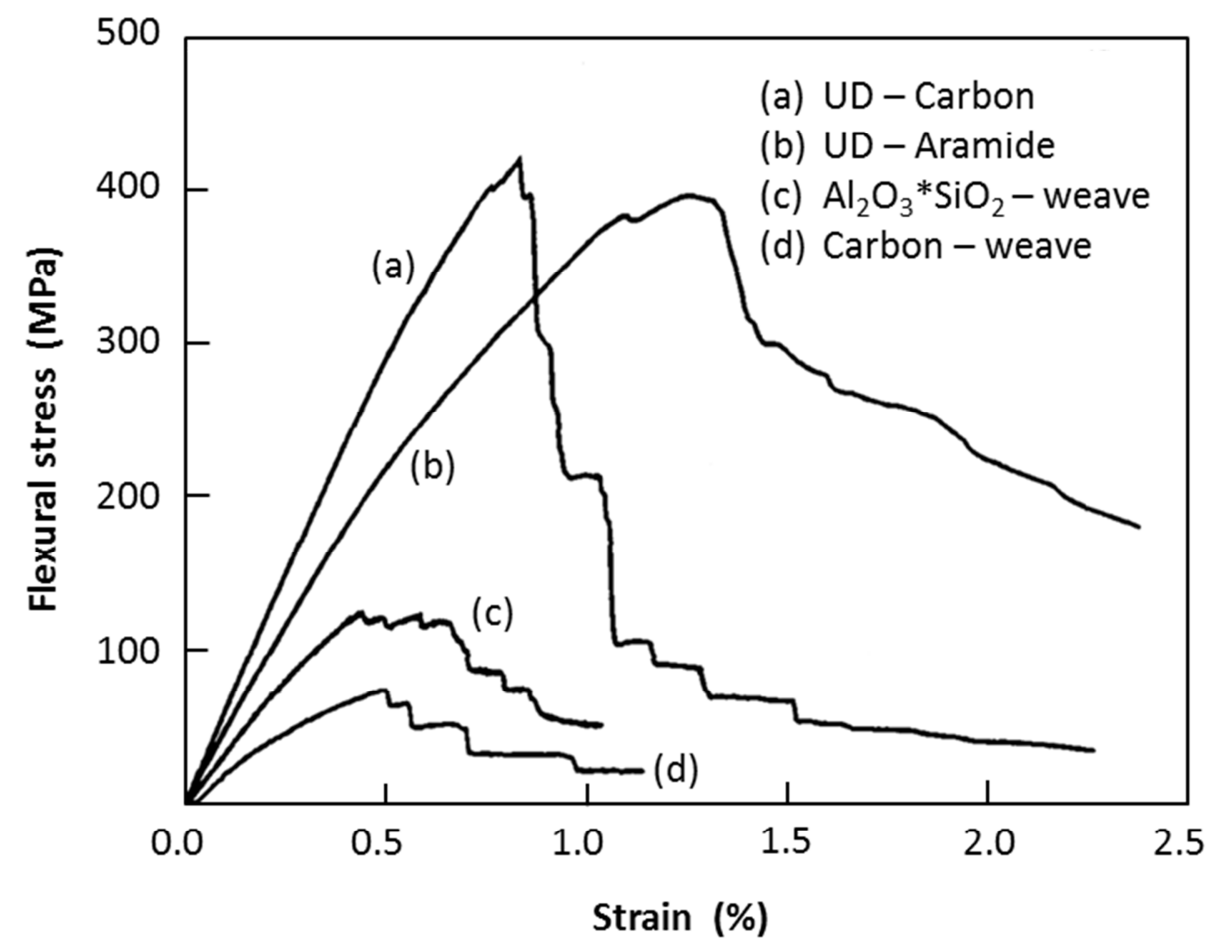

Figure 2.4: Stress-strain curves of fabric (c, d; $V_{\mathrm{f}}=60$ vol.- $\left.\%\right)$ and unidirectional (UD) $\left(a, b ; V_{f}=70\right.$ vol.- $\left.\%\right)$ fibre reinforced geopolymer composites after Foerster $[3,5]$ 
temperature [9]. All of these heating experiments were carried out in air atmosphere with an annealing time of one hour. The effect of heat treatment of 3 and $6 \mathrm{~mm}$ thick composites on the flexural strength and modulus is shown in Figure 2.5. The lower residual strength of the $3 \mathrm{~mm}$ samples was attributed to some degree of fibre oxidation and burnout above $500-600^{\circ} \mathrm{C}$, indicating that the matrix cannot completely protect the fibres from oxygen. Fibre oxidation on the sample surface has a more pronounced effect on the strength of the smaller samples than for the larger samples [9].

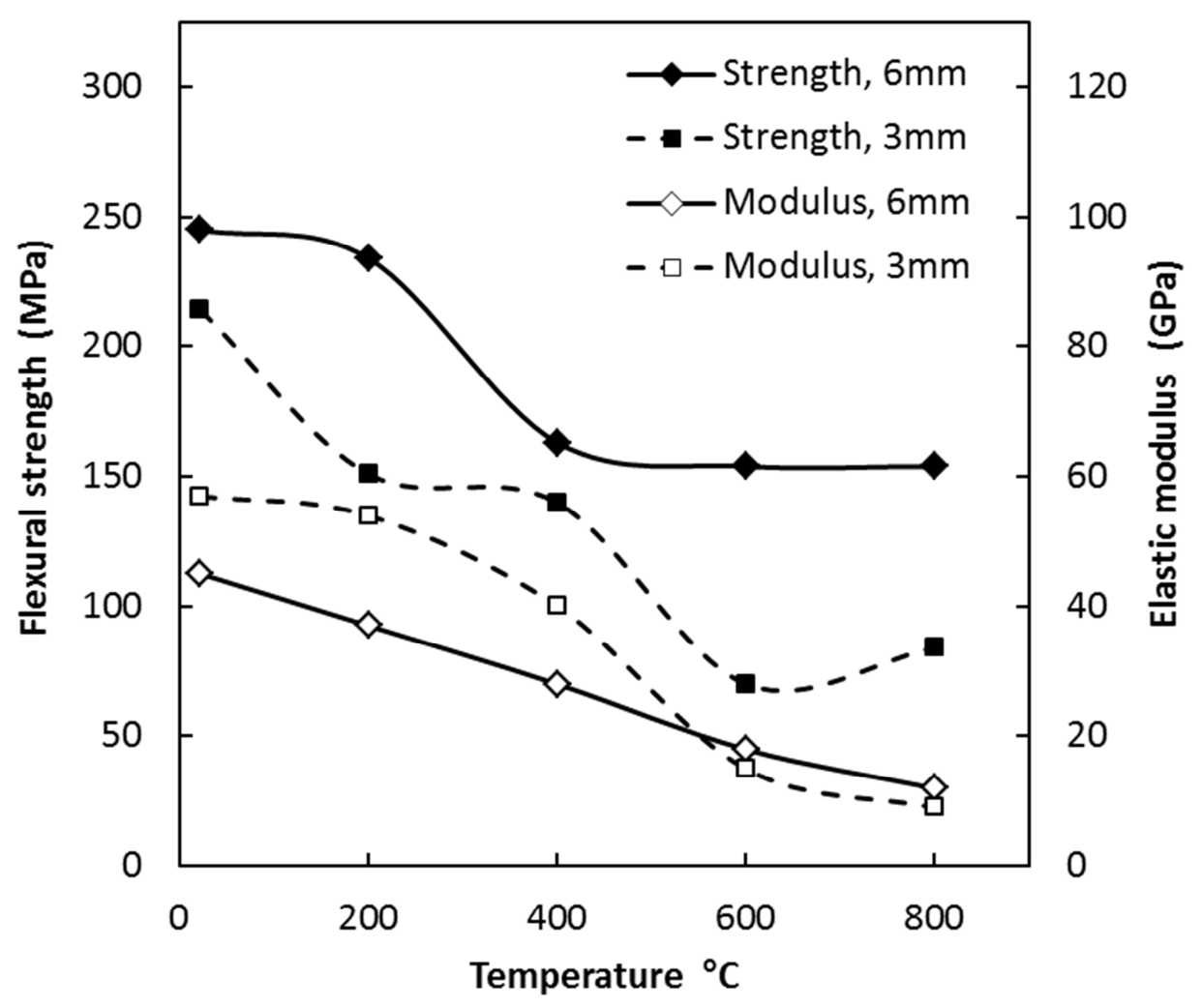

Figure 2.5: Residual strength and modulus of 3 and $6 \mathrm{~mm}$ thick $3 \mathrm{k}$ carbon fabric reinforced geopolymer composites after heat treatment in air for one hour, after Foden [9] and Lyon et al. [7]

Despite the fact that the highest flexural strengths of fabric reinforced composites were obtained with carbon fibres, investigations by Foerster indicate that other reinforcing fibre types are superior to carbon fibres, essentially due to better fibre infiltration and wetting, see Figure 2.4 and Table 2.3 [3]. In particular, aluminosilicate-based fibres were found to improve the strength significantly in 
comparison with carbon fibres. However, aluminosilicate fibres containing $\mathrm{B}_{2} \mathrm{O}_{3}$ showed major fibre degradation whereas carbon and mullite fibres were chemically stable [3]. The use of aluminosilicate fibre fabrics containing small amounts of $\mathrm{B}_{2} \mathrm{O}_{3}$ (Nextel 440) was also studied by Radford et al. [19]. These authors heated the sized fibres at $700^{\circ} \mathrm{C}$ in both air and nitrogen to produce clean desized and carbon-coated fibres, respectively. A maximum flexural strength of $97 \mathrm{MPa}$ was determined by three-point bending test for samples containing 47 vol \% of carbon-coated fibres, compared with $64 \mathrm{MPa}$ for samples containing clean fibres. These strengths were found to decrease to 41 and $37 \mathrm{MPa}$, respectively after heating at $900^{\circ} \mathrm{C}$ for $10 \mathrm{~h}$ in air. The failure mode for the samples containing the desized fibres was reported to be brittle, whereas a degree of fibre pullout was observed for the carbon coated fibres. In contrast to studies by Foden [9] and Hammell [11], all these samples were reported to show a tensile failure mode [19].

Table 2.4: Maximum flexural properties of selected unidirectional fibre reinforced geopolymer composites at room temperature

\begin{tabular}{lccccc}
\hline Fibre type & $\begin{array}{c}\text { Fibre } \\
\text { content }\end{array}$ & $\begin{array}{c}\boldsymbol{\sigma}_{\mathbf{f}, \mathbf{m a x}} \\
\mathbf{( M P a )}\end{array}$ & $\begin{array}{c}\mathbf{E}_{\mathbf{f}, \mathbf{m a x}} \\
\mathbf{( G P a )}\end{array}$ & $\begin{array}{c}\mathbf{W o F} \\
\left(\mathbf{k J} / \mathbf{m}^{\mathbf{2}}\right)\end{array}$ & Reference \\
\hline Carbon & $70 \mathrm{vol} \%$ & 425 & 56 & 15.3 & {$[5]$} \\
Aramid & $70 \mathrm{vol} \%$ & 402 & 37 & 19.9 & “ \\
Carbon & $\mathrm{n} / \mathrm{a}$ & 511 & 93 & $\mathrm{n} / \mathrm{a}$ & {$[9]$} \\
Carbon & $\mathrm{n} / \mathrm{a}$ & 527 & 85 & $\mathrm{n} / \mathrm{a}$ & {$[11]$} \\
Carbon + E-glass & $\mathrm{n} / \mathrm{a}$ & 504 & 80 & $\mathrm{n} / \mathrm{a}$ & “ \\
Carbon & $55 \mathrm{wt} \%$ & 283 & 47 & $\mathrm{n} / \mathrm{a}$ & {$[18]$} \\
AR-glass & $70 \mathrm{wt} \%$ & 255 & 34 & $\mathrm{n} / \mathrm{a}$ & “ \\
Carbon & $20 \mathrm{vol} \%$ & 133 & 37 & 3.9 & {$[13]$} \\
\hline
\end{tabular}

The flexural strengths obtained with unidirectional (UD) fibre composites are significantly greater than those of fabric reinforced composites, as can be seen in Table 2.4 and Figure 2.4. However, this comes at the cost of a high degree of anisotropy which results in a strong dependence of their properties on the loading direction. Maximum three-point flexural strengths $>500 \mathrm{MPa}$ have been reported for UD carbon fibre reinforced geopolymer composites [9, 11]. Although the strength of UD carbon composites measured by Foerster is lower, this author observed that UD aramid fibre composites could obtain similar strength values to carbon fibre 
composites, see Figure 2.4 [3]. However, the lower stiffness of the aramid fibres clearly reflects in the reduced stiffness of the composite. Similar behaviour was observed by Hammell for composites made of a combination of both unidirectional carbon fibres and E-glass fabric [11]. Despite the significantly lower strength and brittle failure that was obtained with all E-glass fibre fabric composites (Table 2.3), composites with a combination of both fibre types obtained similar strengths to the all carbon fibre composites but showed decreased stiffness according to the rule of mixture. The same author also compared the properties of $0^{\circ} / 90^{\circ}$ lay-ups of unidirectional carbon fibres with lay-ups of $3 \mathrm{k}$ carbon fabrics. UD composite were found to show significantly higher strength and strain to failure but reduced stiffness due to better resistance to interlaminar shearing in the woven fabric composites [11]. A number of other mechanical properties including tensile, compression and interlaminar shear strength have been determined by Foden for UD carbon and 3k carbon fabric composites [9]. These results are summarised in Table 2.5.

Table 2.5: Comparison of various mechanical properties of unidirectional and $3 \mathrm{k}$ fabric carbon fibre reinforced geopolymer composites at room temperature, after Foden [9]

\begin{tabular}{lcc}
\hline $\begin{array}{l}\text { Mechanical } \\
\text { properties }\end{array}$ & $\begin{array}{c}\text { UD } \\
\text { carbon }\end{array}$ & $\begin{array}{c}\text { 3k fabric } \\
\text { carbon }\end{array}$ \\
\hline Tension & & \\
$\quad$ Strength [MPa] & 623 & 332 \\
$\quad$ Modulus [GPa] & 156 & 76 \\
Compression & & \\
$\quad$ Strength [MPa] & 105 & 54 \\
$\quad$ Modulus [GPa] & 163 & 102 \\
Flexure & & \\
$\quad$ Strength [MPa] & 511 & 245 \\
$\quad$ Modulus [GPa] & 93 & 45 \\
Interlaminar shear & & \\
$\quad$ Strength [MPa] & $\mathrm{n} / \mathrm{a}$ & 14 \\
$\quad$ & & \\
In-plane shear & & 30 \\
$\quad$ Strength [MPa] & $\mathrm{n} / \mathrm{a}$ & \\
\hline
\end{tabular}




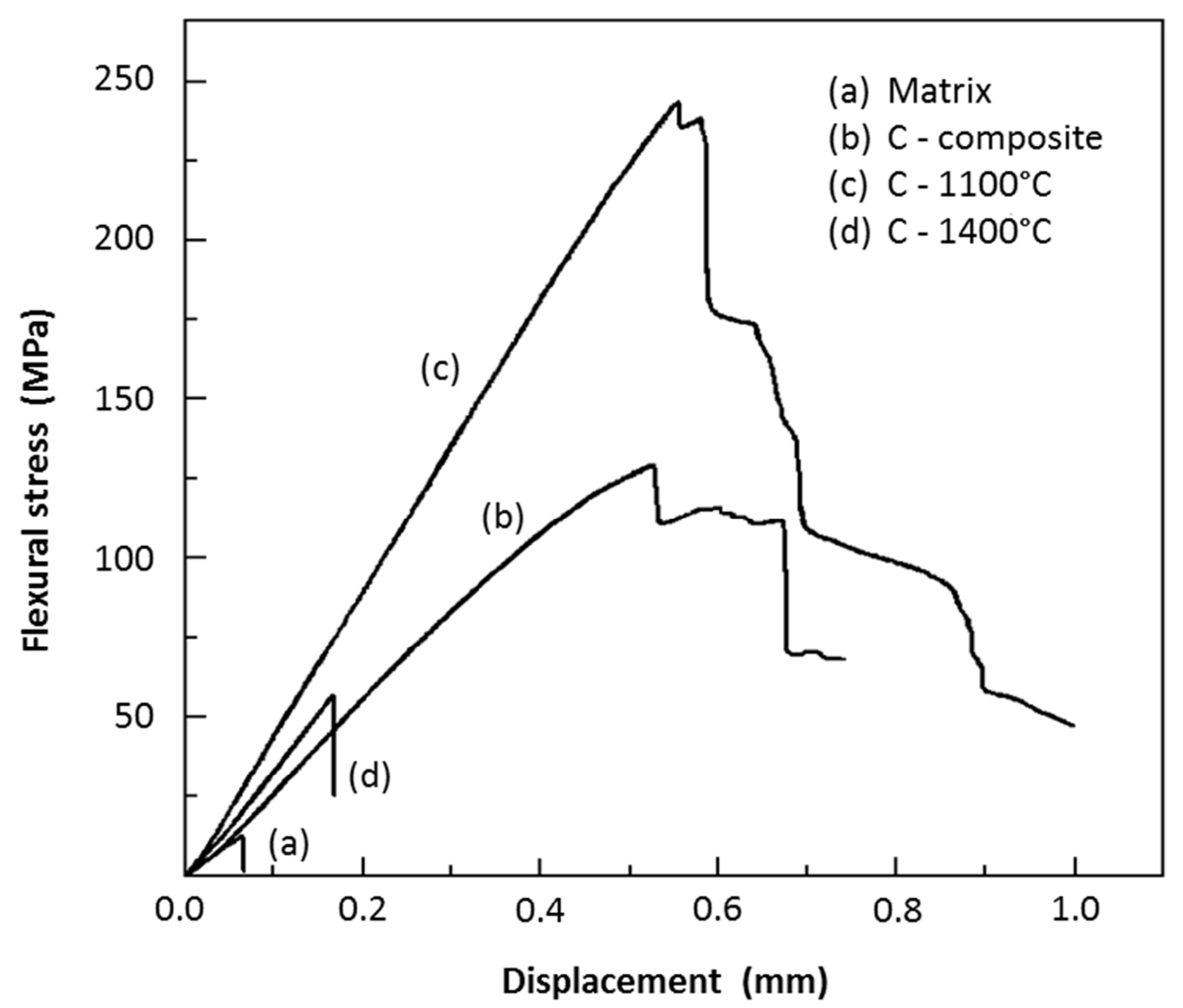

Figure 2.6: Stress-strain curves of geopolymer and unidirectional carbon fibre reinforced geopolymer composites before and after heat treatment, after He et al. [13]. The fibre volume content of unheated composites is $20-25 \%$.

The development of flexural strength and work of fracture of UD carbon composites containing 20-25 volume per cent fibres after high temperature exposure in argon atmosphere was investigated by $\mathrm{He}$ et al. [13]. These authors heat treated composite specimens at various temperatures up to $1400^{\circ} \mathrm{C}$ for 90 minutes. He et al. [13] observed that the initial flexural strength and modulus of $133 \mathrm{MPa}$ and $37 \mathrm{GPa}$ respectively, decreased after heat treatment at $1000^{\circ} \mathrm{C}$ but reached a maximum of $234 \mathrm{MPa}$ and $64 \mathrm{GPa}$ after heating to $1100^{\circ} \mathrm{C}$, respectively. The work of fracture also increased slightly to its maximum value after heat treatment at $1100^{\circ} \mathrm{C}$. The matrix of these composites was found to remain largely amorphous below $1000^{\circ} \mathrm{C}$ but formed leucite and smaller amounts of kalsilite above $1100^{\circ} \mathrm{C}$ [13]. At higher temperatures the strength, elastic modulus and work of fracture decreased gradually with increasing temperature up to $1300^{\circ} \mathrm{C}$. A drastic decrease of the flexural properties was observed after heating to $1400^{\circ} \mathrm{C}$. Non-catastrophic failure was reported in all these composites except for samples heated to $1400^{\circ} \mathrm{C}$. The 
development of the stress-strain behaviour after heat treatment and the corresponding electron micrographs of the fracture surfaces are presented in Figure 2.6 and Figure 2.7, respectively. The fracture surface of the unheated composite (Figure 2.7a) shows almost no fibre fracture. Instead, fibre debonding and matrix fragmentation can be seen around the fibre bundles, indicating a weak fibre-matrix interface and typical behaviour for weak matrix composites [13]. Similar features are observed in samples heated to $1000^{\circ} \mathrm{C}$. On the other hand, composites heated at $1100^{\circ} \mathrm{C}$ show long fibre pull-out and fibre fracture (Figure 2.7b). The increased fibre-matrix bond strength due to significant shrinkage of the matrix in samples heated at this temperature results in an optimal strength and non-catastrophic failure. Similar behaviour is observed for heat treatment up to $1300^{\circ}$. However, increasing temperatures result in shorter pullout lengths. This indicates a further increase in the fibre-matrix bond strength. At $1400^{\circ} \mathrm{C}$, strong fibre-matrix interactions were found to lead to a
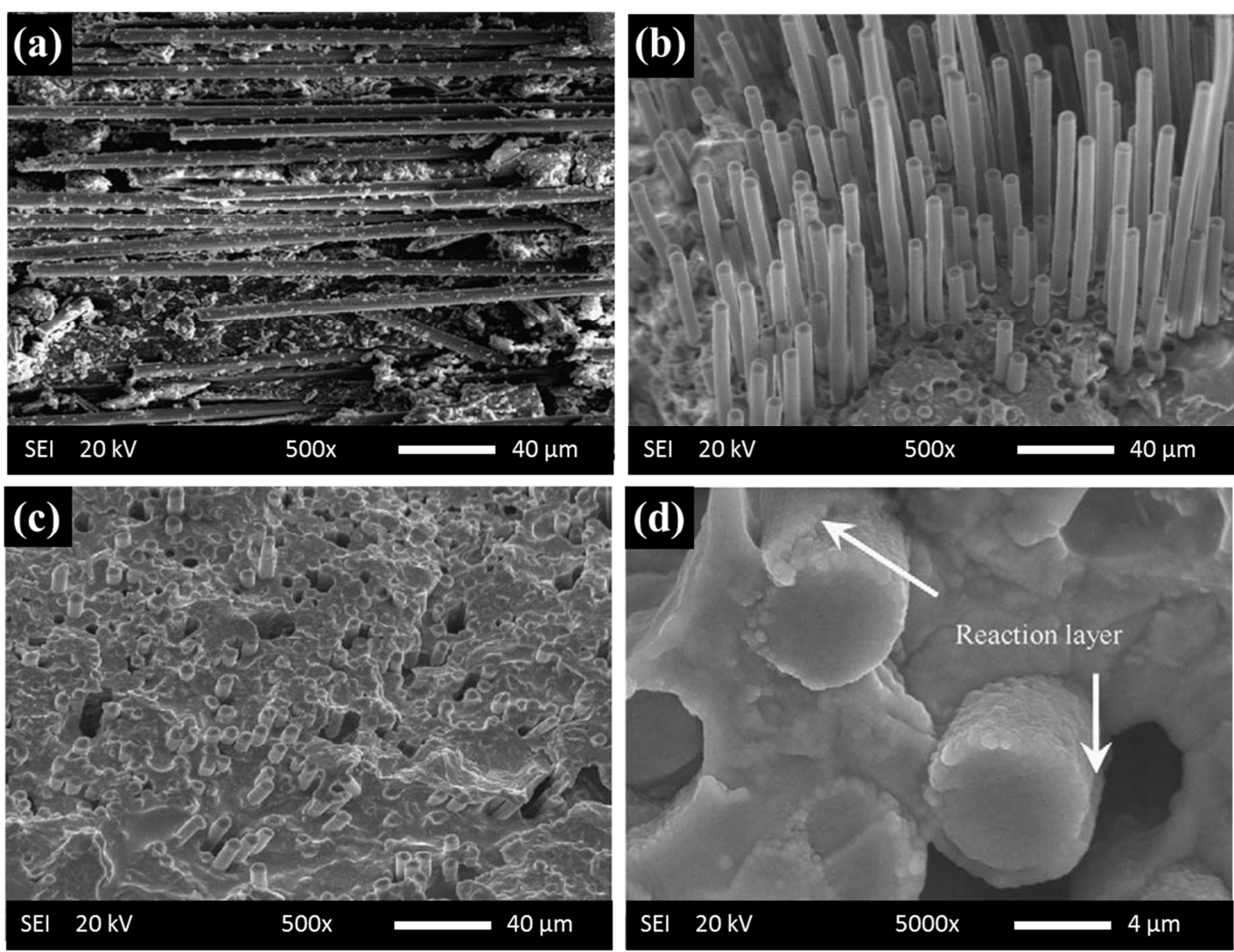

Figure 2.7: Fracture surfaces of unidirectional carbon fibre reinforced geopolymer composites: (a) before heat treatment, (b) heated at $1100^{\circ} \mathrm{C}$, (c) heated at $1400^{\circ} \mathrm{C}$, (d) interface reaction after heating at $1400^{\circ} \mathrm{C}$ in argon for 90 minutes, after He et al. [13] 
substantial degradation of the carbon fibres and the formation of a thick layer of $\mathrm{SiC}$ (Figure $2.7 \mathrm{c}, \mathrm{d}$ ), resulting in high interfacial bond strength and brittle failure of the composite [13]. The microstructural evolution of the fibre-matrix interface in these composites is studied in more detail by the same authors in [15]. It is concluded that the increasing fibre-matrix interface reaction at temperatures above $1200^{\circ} \mathrm{C}$ causes the gradual transformation of the purely physical bond between the carbon fibres and the matrix below $1100^{\circ} \mathrm{C}$ to a strong chemical bond, leading to an increasingly brittle composite behaviour between $1200-1400^{\circ} \mathrm{C}[15]$.

\subsection{Scope of thesis}

One of the main attractions of geopolymers is their combination of low temperature processing and properties, in particular their thermal stability. This material profile essentially combines two of the main advantages of both polymer and ceramic materials, making geopolymers a prime candidate as new matrix materials for the development of more cost-efficient CMC-like materials for applications up to around $800^{\circ} \mathrm{C}$. Although the existing literature suggests a significant potential of geopolymer matrix composites for structural applications, they have so far received relatively little attention. One of the main reasons for this may be associated with a general scepticism against geopolymer science and technology in the past. However, geopolymer technology has established itself in recent years as a growing area of scientific interest and significant advances towards a better understanding of geopolymers have been made since the first studies on geopolymer composites. Therefore, a further investigation of the idea of geopolymer matrix composites seems of considerable interest.

As mentioned in the introductory chapter, the aim of this study is to investigate several key aspects of fibre reinforced geopolymer matrix composites in order to improve the basic understanding of these materials and to evaluate their general potential as cost-efficient composite materials. Therefore, the objective of this work is rather broad, trying to analyse a number of general aspects of geopolymer 
composites that may not just be specific to the samples tested here but also transferable to other geopolymer composites. This work can be generally divided into four main parts:

(i) characterisation of a model geopolymer matrix system

(ii) investigation of the effects of various parameters on the mechanical properties and failure behaviour of unidirectional fibre reinforced composites

(iii) analysis of fibre-matrix interactions

(iv) initial ion exchange experiments and general considerations regarding the use of geopolymer matrix composites as precursors for CMCs

An abundant number of raw materials can generally be used for the production of geopolymers. This, however, goes along with several advantages and disadvantages as discussed in chapter 2.2.3. One of the major disadvantages is often the lack of comparability between different studies. Although several relatively standard matrix systems and composition ranges have evolved over the years (see optimum composition range, chapter 2.2.3), there is little restriction on the purity, particle size, composition, morphology, type of alkali activator solution, etc. of the starting materials. Thus, a single, well defined model geopolymer matrix system is difficult to identify. Based on a number of considerations such as the relative simplicity and cost of the component system and previous studies by the geopolymer group at Victoria University, a sodium activated metakaolin-type component system that could produce a variety of geopolymers largely within the suggested optimal composition range was chosen for this study to form a system as close to any potential model system as possible. Several geopolymer compositions were initially evaluated in order to identify a suitable matrix composition for the composite fabrication. The matrices were characterised mainly by mechanical strength testing a basic microstructural analysis of the different geopolymer matrices was also carried out. The initial characterisation and properties of the unreinforced geopolymer matrices are discussed in chapter 4. 
The state of the art of geopolymer composites was reviewed in detail in the previous section. Most of these studies focused on the use of various types and forms of carbon fibres. Although some other fibre types have also been investigated, the results are sometimes based on as little as one measurement. Also, the use of different materials, fabrication processes and test methods makes it extremely difficult to compare individual results. Therefore, the aim of this study was to investigate a range of different variables on samples all subject to the same processing parameters in order to allow direct comparison between the different effects. Unidirectional fibre reinforced composites were fabricated using basalt, carbon and alumina fibres. Basalt, carbon and alumina fibres represent three very different kinds of fibres not just in terms of chemical, thermal and mechanical properties but also from an economical point of view. Since one of the main advantages of geopolymer composites would be their cost-efficiency in the absence of resource or energy intensive processing and fabrication steps or other cost adding contributions, the fibres become the main cost factor of the composite. Therefore, the relation between performance and cost of the fibre/composite has to be considered. The mechanical properties of unidirectional fibre reinforced geopolymer composites are investigated in chapter 5. Besides the effect of the fibre type, several other parameters including fibre sizing, specimen dimensions, drying time and matrix composition on the flexural properties and the failure behaviour of the composites were studied. For reasons of cost and fibre availability, some effects were only studied exemplarily on basalt fibre composites.

Microstructural aspects of geopolymer composites, in particular the interaction between fibre and matrix across the interface, have so far hardly been investigated. However, the interaction between fibre and matrix is an important factor for the behaviour of composite materials. Therefore, electron microscopy study of the interfaces was one of the main objectives of this work. The fibre-matrix interaction was studied on polished and fractured surfaces after heating to various temperatures up to $1100^{\circ} \mathrm{C}$ to evaluate the general behaviour of the different composite types at higher temperatures. X-ray diffraction was used to determine the formation of 
crystalline phases. The results of the microstructural investigation of the composites are presented and discussed in chapter 6 .

The heating experiments were also used as a basis for evaluating the general potential of geopolymer composites as precursors for CMCs. In this context, the idea of ion exchanging composite bars in order to influence the phase formation at higher temperatures was explored in a small side project. Although a detailed investigation of this subject was beyond the scope of this study, some preliminary results will be presented in chapter 7 and the potential of geopolymer composite precursors will be discussed. 


\section{Chapter 3}

\section{Materials and experimental methods}

\subsection{Starting materials}

\subsubsection{Geopolymer matrix system}

All matrix compositions in this study were derived from the same four component system including dehydroxylated halloysite as the aluminosilicate source, a commercially available sodium silicate solution, sodium hydroxide pellets and distilled water. The halloysite was provided by Imerys Tableware (NZCC Halloysite Premium, Grade: Ultrafine $-H$ ) and is a very high purity New Zealand clay. Typical thermal analysis and particle size data of the halloysite are presented in Figure 3.1.
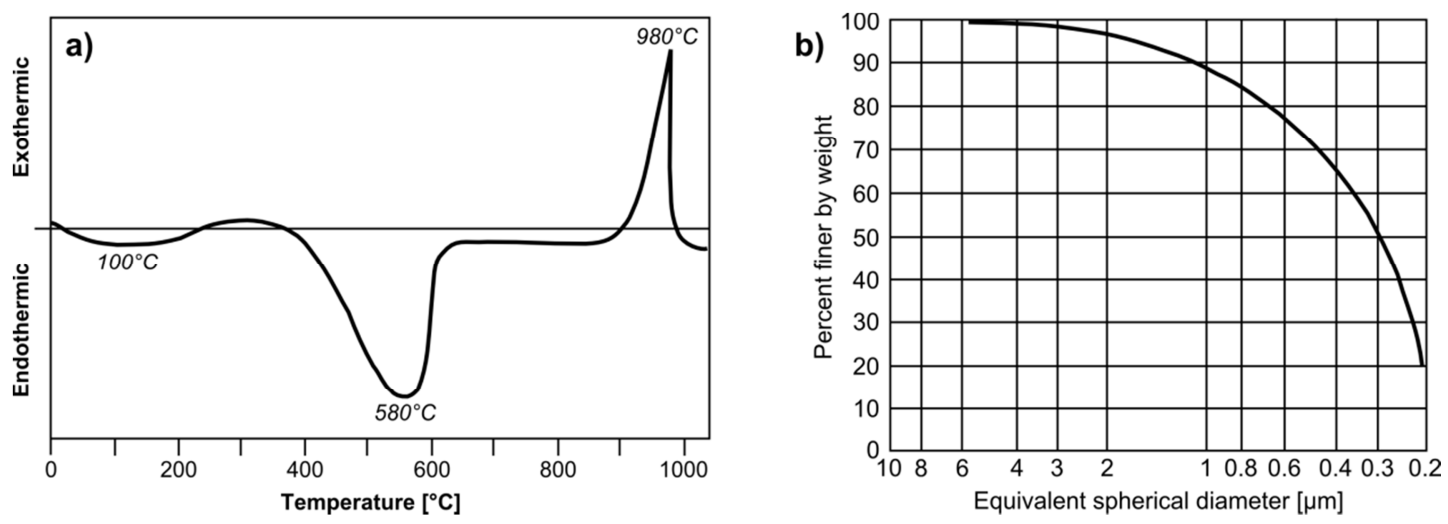

Figure 3.1: Typical DTA curve (a) and particle size distribution (b) of NZCC Halloysite Premium as specified by supplier 

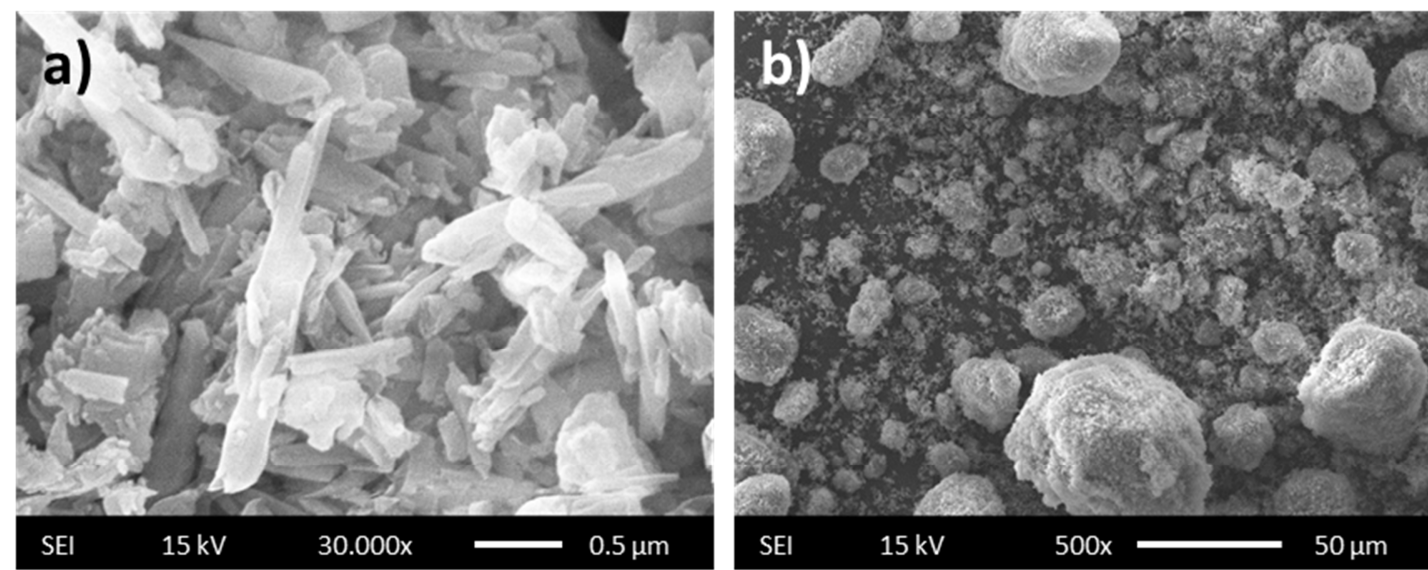

Figure 3.2: Morphology of dehydroxylated halloysite after calcination at $600^{\circ} \mathrm{C}$ over night and screening on $353 \mu \mathrm{m}$ sieve: a) high and b) low resolution SEM micrograph

The as-received powder was dehydroxylated at $600^{\circ} \mathrm{C}$ over night and subsequently screened on a $353 \mu \mathrm{m}$ (44 mesh) sieve to ensure that no large agglomerates or other artefacts were present in the calcined powder. The typical morphology of the dehydroxylated halloysite powder at high and low magnification is shown in Figure 3.2. The majority of the individual dehydroxylated halloysite particles showed a size $<1 \mu \mathrm{m}$ (Figure 3.2(a)). However, particle agglomerates up to about $50 \mu \mathrm{m}$ in diameter could be observed at lower magnification, see Figure 3.2(b). The chemical composition of major oxides of the dehydroxylated halloysite, determined by x-ray fluorescence (XRF) analysis, is shown in Table 3.1.

Table 3.1: Chemical analysis of dehydroxylated halloysite

\begin{tabular}{lcccccccccc}
\hline Oxide & $\mathrm{SiO}_{2}$ & $\mathbf{A l}_{2} \mathrm{O}_{3}$ & $\mathrm{Fe}_{2} \boldsymbol{O}_{3}$ & $\mathrm{TiO}_{2}$ & $\mathrm{CaO}$ & $\mathrm{MgO}$ & $\mathrm{K}_{2} \mathrm{O}$ & $\mathrm{Na}_{2} \mathrm{O}$ & $\mathrm{P}_{2} \mathrm{O}_{5}$ & $\mathrm{LOI}$ \\
wt- $\%$ & 56.61 & 40.96 & 0.34 & 0.08 & 0.01 & 0.02 & 0.01 & $<0.01$ & 0.19 & 1.43 \\
\hline
\end{tabular}

Dehydroxylated halloysite has successfully been used as the standard aluminosilicate source for metakaolin-based geopolymers in the geopolymer research group at Victoria University in recent years and its use was therefore continued in the present study. Halloysite is a kaolin-type clay mineral that shows near identical chemical composition to the more common kaolinite except that it contains additional water molecules between the layers. In hydrated form, halloysite most commonly exhibits a tubular morphological appearance compared to the typical plate-like structure of 
kaolinite. However, the tubular structure is not retained after dehydroxylation as indicated by electron microscopy analysis (see Figure 3.2). Therefore, no major differences in the reaction behaviour between this aluminosilicate source and other types of metakaolinite are expected. In analogy to metakaolinite, the dehydroxylated halloysite may be subsequently also referred to as metahalloysite.

A combination of SodSil D sodium silicate solution (Orica Chemnet, Grade D, $\mathrm{SiO}_{2} / \mathrm{Na}_{2} \mathrm{O} \sim 2$ 2, 29.4 wt.-\% $\mathrm{SiO}_{2}$ ) and sodium hydroxide pellets (> $98 \%$ purity, various suppliers) were used as alkali activators.

\subsubsection{Fibres}

Three types of inorganic fibres, i.e. basalt, carbon and alumina fibres, were investigated as potential reinforcements for geopolymer matrices. All of these fibre types were used in form of continuous multifilament tows to fabricate unidirectional fibre reinforced composites for mechanical testing. Boron monofilaments were also used in one experiment in combination with basalt fibres. An overview of all fibre types and selected properties are shown in Table 3.2.

Table 3.2: Investigated fibres and relevant properties as specified by the supplier

\begin{tabular}{|c|c|c|c|c|c|}
\hline $\begin{array}{l}\text { Supplier/ } \\
\text { Fibre }\end{array}$ & $\begin{array}{l}\text { Density } \\
\left(\mathrm{g} / \mathrm{cm}^{3}\right)\end{array}$ & $\begin{array}{c}\text { Diameter } \\
(\mu \mathrm{m})\end{array}$ & $\begin{array}{c}\text { Fibres/ } \\
\text { tow }\end{array}$ & $\begin{array}{l}\text { Elastic } \\
\text { modulus } \\
(\mathrm{GPa})\end{array}$ & $\begin{array}{l}\text { Tensile } \\
\text { strength } \\
\text { (MPa) }\end{array}$ \\
\hline $\begin{array}{l}\text { R\&G Faserverbundwerkstoffe } \\
\text { Basalt } 2400 \text { tex }\end{array}$ & 2.75 & $\sim 13$ & $5-6000$ & 89 & 4840 \\
\hline $\begin{array}{l}R \& G \text { Faserverbundwerkstoffe } \\
\text { Carbon } 1600 \text { tex, } 24 \mathrm{k} \\
\text { Tenax }^{\circledR} \text { E HTS } 40\end{array}$ & 1.77 & 7 & 24000 & 240 & 4300 \\
\hline $\begin{array}{l}3 M \\
\text { Alumina } \\
\text { Nextel } 610\end{array}$ & 3.9 & $10-12$ & 750 & 380 & 3100 \\
\hline $\begin{array}{l}\text { Specialty Materials } \\
\text { Boron, monofilament }\end{array}$ & 2.5 & 102 & $\mathrm{n} / \mathrm{a}$ & 400 & 3600 \\
\hline
\end{tabular}


The chemical composition of the basalt fibre, determined by XRF analysis, is given in Table 3.3. All multifilament fibres were provided in sized form. According to the supplier information, the carbon fibres had an epoxy-based sizing and the alumina fibres had a PVA-based sizing. The chemical nature of the sizing of the basalt fibres was not disclosed by the supplier.

Table 3.3: Chemical analysis of basalt fibre

\begin{tabular}{lccccccccc}
\hline Oxide & $\mathrm{SiO}_{2}$ & $\mathrm{Al}_{2} \mathrm{O}_{3}$ & $\mathrm{Fe}_{2} \mathrm{O}_{3}$ & $\mathrm{TiO}_{2}$ & $\mathrm{CaO}$ & $\mathrm{MgO}$ & $\mathrm{K}_{2} \mathrm{O}$ & $\mathrm{Na}_{2} \mathrm{O}$ & $\mathrm{P}_{2} \mathrm{O}_{5}$ \\
$\mathrm{wt}-\%$ & 54.58 & 17.51 & 9.80 & 1.10 & 8.02 & 3.91 & 1.66 & 2.44 & 0.19 \\
\hline
\end{tabular}

Unlike typical ceramic composites, fibre degradation due to high processing temperature is not an issue with geopolymer composites. However, geopolymer binders are synthesised in a highly alkaline environment. These conditions may lead to significant fibre corrosion and degradation. Therefore, the chemical stability of all fibres under highly alkaline condition was investigated in a preliminary experiment. Small amounts of fibres were exposed to a concentrated $\mathrm{NaOH}$ solution $(12.5 \mathrm{M})$ for $150 \mathrm{~h}$ at $40^{\circ} \mathrm{C}$. The concentration of the $\mathrm{NaOH}$ solution was identical to the one used for the fabrication of geopolymer composition M1, see chapter 3.2. The fibres were subsequently washed, dried and inspected under the electron microscope for any change in surface morphology that may indicate a potential loss of fibre strength due to corrosive attack under the highly alkaline conditions (see chapter 6.1.1).

\subsection{Composition and processing of geopolymer binders}

Four compositions (M1 - M4) with varying silica-to-alumina ratio, alkali and water content were chosen after preliminary trials of various compositions within the matrix system for further investigation with the aim to evaluate the most suitable mixture for the fabrication of geopolymer composites. After initial evaluation of the different mixtures, M1 was chosen as the main matrix composition for the fabrication of composites. A somewhat optimised composition (M5) was introduced at a later stage of the project. However, M5 matrix compositions were only applied in a small 
number of composites for comparative purposes. Other matrix compositions were not used for the fabrication of composites. With the exception of M3, all compositions were largely within the standard compositional range for geopolymers, as described in chapter 2.2.3, and should therefore achieve good and well-reacted geopolymers. An overview of the composition and respective molar ratios of all five mixtures are presented in Table 3.4 .

Table 3.4: Component mixture and estimated molar ratios of investigated compositions

\begin{tabular}{lccccc}
\hline Component (g) & M1 & M2 & M3 & M4 & M5 \\
\hline Metahalloysite & 80 & 80 & 80 & 80 & 80 \\
SodSil D & 40 & 60 & 40 & 40 & 80 \\
Dist. water & 40 & 40 & 40 & 50 & 24 \\
$\mathrm{NaOH}$ & 20 & 20 & 10 & 20 & 12 \\
\hline & & & & \\
Molar ratio & & & & & \\
\hline $\mathrm{SiO}_{2} / \mathrm{Al}_{2} \mathrm{O}_{3}$ & 3.0 & 3.3 & 3.0 & 3.0 & 3.6 \\
$\mathrm{Na}_{2} \mathrm{O} / \mathrm{SiO}_{2}$ & 0.36 & 0.37 & 0.23 & 0.36 & 0.30 \\
$\mathrm{H}_{2} \mathrm{O} / \mathrm{Na}_{2} \mathrm{O}$ & 10.1 & 10.5 & 15.8 & 11.7 & 11.3 \\
\hline
\end{tabular}

Preliminary small quantity mixtures were prepared by hand mixing of metahalloysite and a pre-dissolved alkali activator solution. However, to improve reproducibility and homogenisation of larger amounts of geopolymer binder, a mechanical mixer was used (Figure 3.3). In a first step, sodium hydroxide was dissolved in distilled water in a closed container. Due to the generation of significant heat during this exothermic dissolution reaction, the solution was subsequently cooled down in ice water to about room temperature. The $\mathrm{NaOH}$-solution was then transferred into the pre-cooled mixing container and mixed with SodSil D sodium silicate solution to form a homogeneous alkali activator solution. Subsequently, the metahalloysite was slowly added under constant stirring at about $250 \mathrm{rpm}$. The mixture was further homogenised for about five minutes before use. Keeping the geopolymer mixture at or below room temperatures during the mixing process was favourable in order to avoid pre-mature setting of the viscous binder. Therefore, the initial cooling of the $\mathrm{NaOH}$ solution was necessary. The pre-cooling of the mixing container further helped to reduce heating up of the mixture during the subsequent process. 
All mixtures were standardised to a batch size of $80 \mathrm{~g}$ metahalloysite which provided a sufficient amount of geopolymer binder to mould a minimum of five bars and cylinders for flexural and compressive testing, respectively. The mixer also showed the best performance for that amount of material. $40 \mathrm{~g}$ metahalloysite mixtures tended to leave a dry, poorly homogenised patch on the bottom of the mixing container. The chosen speed of about $250 \mathrm{rpm}$ was the slowest speed possible with the available stirrer. Faster speeds or much larger amounts of geopolymer binder were limited by the height and potential overflowing of the mixing container.

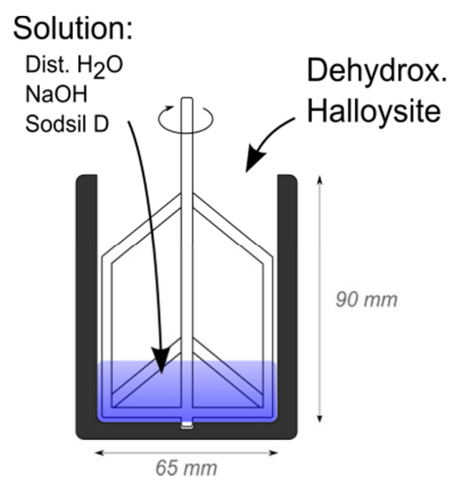

Figure 3.3: Schematic presentation of the mixer design developed and used in this work

\subsection{Fabrication of unreinforced specimens}

Unreinforced specimens were produced in a slip casting process by pouring the readily homogenised geopolymer binder into plastic moulds and subsequent curing. Three different types of unreinforced specimens were prepared for flexural, compressive and splitting-tensile strength testing, respectively (see Figure 3.4 for mould shapes and sizes). All moulds were greased with a thin layer of Vaseline to facilitate easy removal of the hardened specimens from the moulds after curing. Bars of the size $10 \times 6 \times 120 \mathrm{~mm}$ were produced for flexural strength testing of unreinforced geopolymer specimens. In order to obtain a flat and even top surface, strips of plastic sheet (approx. $20 \times 140 \mathrm{~mm}$ ) were used to directly cover the moulds after filling. The sheets were pressed flat onto the moulds with a spatula, squeezing out any excess binder. The sheets could easily be peeled off after curing and good surface qualities could be achieved, eliminating the need of post-curing surface 
machining. Cylinders for compressive testing were covered in the same way. Pellets for splitting-tensile testing were poured to a height of 10 to $20 \mathrm{~mm}$ and the moulds were covered with either Parafilm or Gladwrap. All samples were additionally placed in sealed plastic bags to prevent excessive water evaporation during curing which can lead to cracking and reduced mechanical strength.

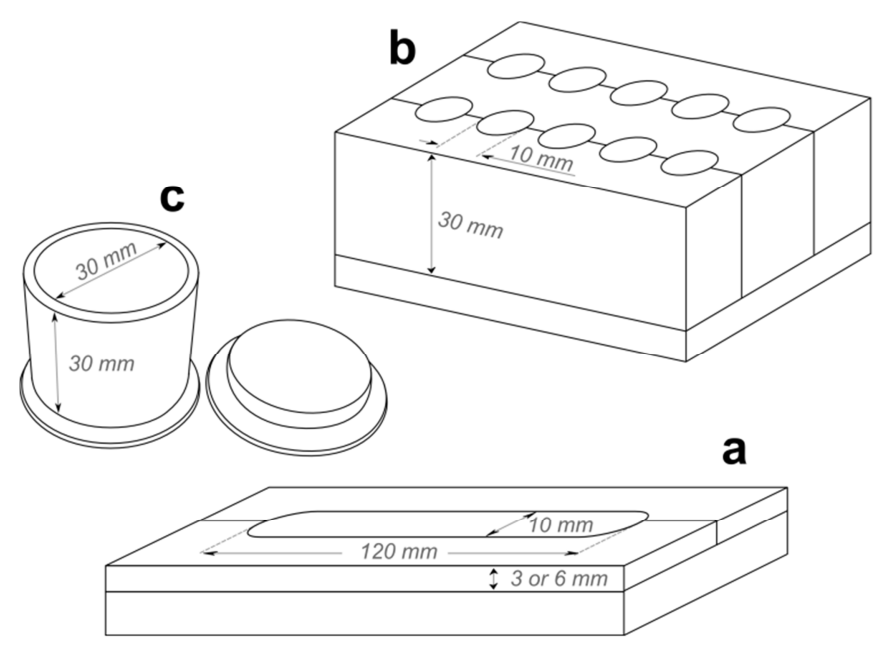

Figure 3.4: Moulds used for the fabrication of a) unreinforced and UD composite bars b) unreinforced cylinders and c) unreinforced pellets

Several curing schedules with varying times and temperatures between 24 to $48 \mathrm{~h}$ and 20 to $80^{\circ} \mathrm{C}$, respectively were tested in preliminary experiments to evaluate the effect of curing conditions on the mechanical strength in order to determine the optimum curing conditions. A splitting-tensile strength method was employed for mechanical characterisation. However, sample cracking during the post-curing drying process was a major issue with these samples and no definite conclusions could be drawn from these experiments. Therefore, the standard curing parameters most commonly used within the geopolymer research group at Victoria University, i.e. $40^{\circ} \mathrm{C}$ for $48 \mathrm{~h}$, were assumed for all following samples throughout this study, including composites. All specimens were de-moulded after the $48 \mathrm{~h}$ curing period and allowed to dry under ambient conditions for typically seven days before testing. 


\subsection{Fabrication of fibre reinforced specimens}

\subsubsection{Preparation of fibre tapes}

Unidirectional fibre reinforced composite bars for mechanical testing were prepared by the hand lay-up of impregnated fibre tapes in plastic moulds. The fibre tapes were prepared from continuous fibre tows. For the purpose of this study, a fibre tape shall be defined as a unidirectional fibre pre-form made of one or more multifilament tows and a width and length of approximately 10 and $150 \mathrm{~mm}$, respectively. The basalt and carbon fibre tows were large enough to cover the $10 \mathrm{~mm}$ width of the bar mould with a single tow, whereas six parallel tows of alumina fibres were needed to cover the same area.

For both basalt and carbon fibres the continuous fibre tows were fixed with adhesive tape at distances of about $150 \mathrm{~mm}$ and subsequently cut across the taped area. The adhesive tape on both ends provided sufficient fixation of the cut fibre tow and allowed for relatively easy handling. The fibre tapes were then pulled several times over a round glass tube with slight sideward movements in order to improve the parallel alignment of the single fibres in the tow and to separate the partially cohering fibres (due to the fibre sizing) for better impregnation of the fibre tapes. However, it has to be noted that the alignment of fibres in the original basalt tow was not very good and smaller fibre bundles in the tow were often intertwined. Despite improvements of the fibre alignment by the method described above, the basalt fibre tapes showed the worst parallelism of the three fibre tapes.

Alumina fibre tapes were prepared in a winding process. The continuous tow was fixed with double-sided adhesive tape to a round glass jar of suitable diameter and six parallel tows were wound onto the glass jar. Double-sided adhesive tape was put across all six tows covering the first tape. The fibre tow was then wound back to the starting point creating a double-layered fibre tape with six tows per layer. The fibre layers were again fixed with (single-sided) adhesive tape before cutting off the continuous tow. The wound up fibre tow was then cut across the layers of adhesive tape with a razor blade and each side peeled off the glass jar to obtain the final 
alumina fibre tape. The process of making alumina fibre tapes was very time consuming. To make this process somewhat more efficient and to reduce the number of fibre tapes that had to be made and laid-up, respectively, the double-layer option was chosen over single-layer alumina fibre tapes.

\subsubsection{Desizing of fibres}

All multifilament fibre types investigated in this study were received in sized form, i.e. with a very thin organic protective coating. The fibre sizing is generally removed during the fabrication process of ceramic composites and often replaced by other coatings such as carbon or boron nitride that act as a weak interface between fibre and matrix. These processes, however, add to the overall cost of the material. Therefore, not having to remove the fibre sizing would contribute to the costefficiency of the composite. Hence, the obvious preference for geopolymer composites would be to use the as-received fibres without further treatment. However, the effects of fibre sizing on the bonding behaviour, the mechanical strength and the failure behaviour of fibre reinforced geopolymer composites have hardly been investigated. Therefore, the behaviour of sized and desized fibres was compared in this study.

A Bunsen burner was used for the desizing of all fibres. Fibre tapes or tows were held with metal clamps on each end and were slowly moved over the flame for about ten seconds on each side. Although this method is rather simple, it had a practical advantage over desizing fibres in a furnace. That is, it allowed the desizing of the already prepared fibre tapes instead of having to prepare fibre tapes from desized tows. Also, the ends of the desized fibre tapes could still be fixed with adhesive tape and therefore handled the same way as sized fibre tapes. Since the basalt fibres could not withstand the temperature of the Bunsen burner, these fibres were only used in sized form. Scanning electron microscopy was used to confirm the successful removal of fibre sizings and to identify any form of possible degradation effects on the fibre surface due to the desizing process, in particular for carbon fibres (see chapter 6.1.1). 


\subsubsection{Fabrication of unidirectional composite bars}

As mentioned before, composite bars were made by hand lay-up of impregnated fibre tapes. The main steps of the general fabrication process are presented in Figure 3.5. The first step was the impregnation of the fibre tapes. For that reason, ten fibre tapes were arranged on a plastic sheet and then thoroughly impregnated individually with geopolymer binder from both sides using a spatula and roller. Unlike the fabrication of unreinforced specimens, only a small amount of geopolymer was taken out of the mixing container for the impregnation of the fibre tapes and the remaining binder was continued to stir. The continued mixing postponed the setting of the mixture and extended the effective use-time of the matrix binder. This way, three composite bars could be produced per batch of geopolymer mixture. The impregnated fibre layers were cut to $115 \mathrm{~mm}$ length and placed in a mould one by one using a spatula and light pressure to place and evenly distribute each fibre layer in the mould. A plastic sheet was used again to directly cover the top surface of the lay-up. Some pressure was applied with the spatula moving over the plastic sheet in order to obtain a smooth and even surface. The moulds were sealed in plastic bags and cured for $48 \mathrm{~h}$ at $40^{\circ} \mathrm{C}$ similar to unreinforced specimens. The demoulded composite bars were allowed to dry for a standard seven days under ambient conditions before testing. Typical dimensions of cured composite bars were $10 \times 115 \mathrm{~mm}$ with a thickness of roughly $3 \mathrm{~mm}$. The fibre volume content was approximately $30 \%$ for all specimens. Five composite bars were tested in each sample series unless stated otherwise.

The fabrication of good quality UD composite bars was relatively difficult and required a fast and skilled way of working. Nevertheless, the reproducibility of specimens was somewhat limited by the all-manual processing. Therefore, some variability between samples has to be expected. A controlled variation of the fibre volume content along with a homogeneous fibre distribution in the composite bar also proved difficult. Thus, several alternatives were tested in preliminary experiments with basalt fibre tapes in order to evaluate parameters for a fabrication standard that promised the best reproducibility and relatively homogeneous fibre distribution. The number of fibre tapes that could be impregnated at a time was 


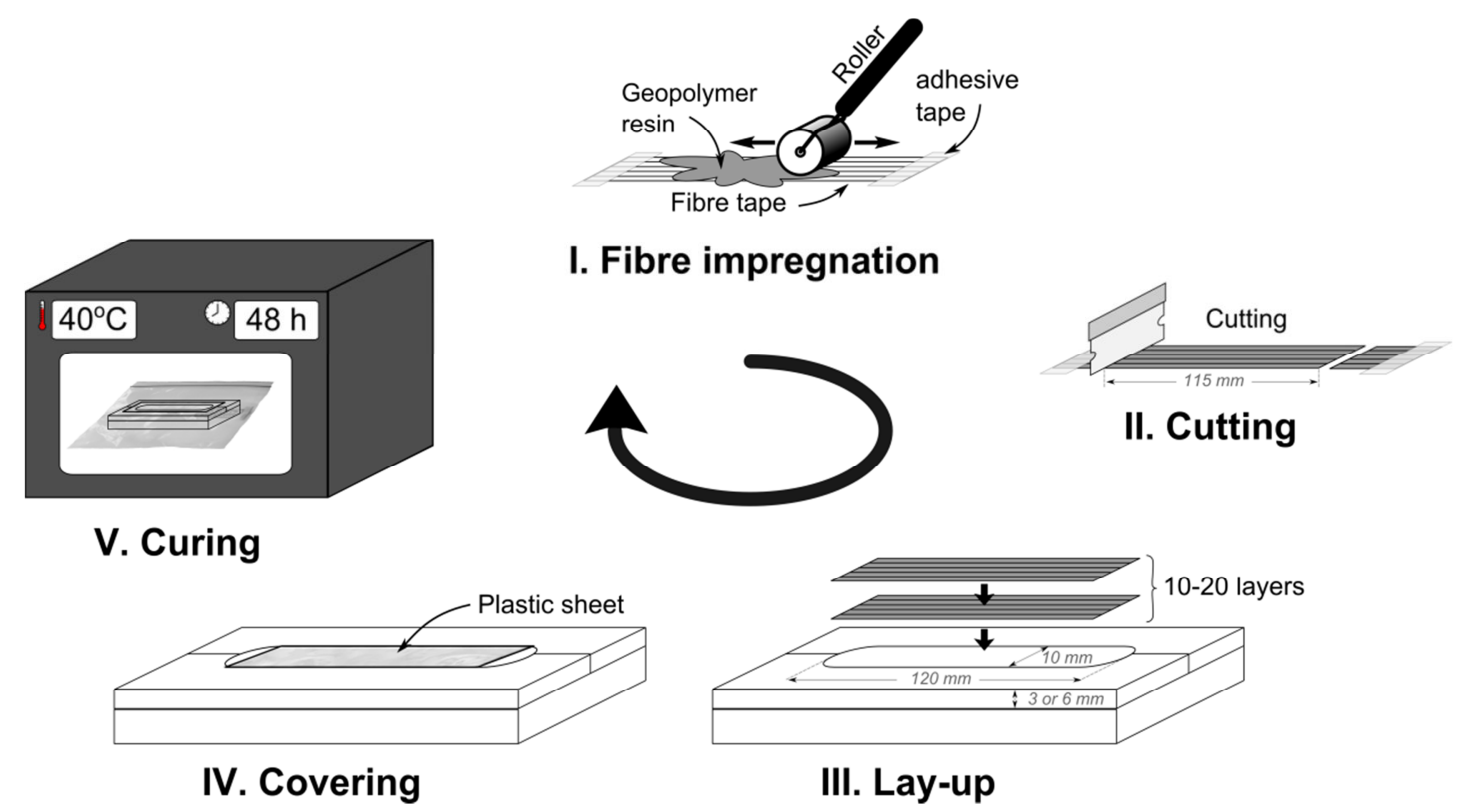

Figure 3.5: Schematic fabrication process for UD fibre reinforced composite bars

limited to about ten. That was due to the relatively small time span between the development of favourable tackiness and dry out of the impregnated fibre tapes. The impregnation of a larger number of fibre tapes at a time led to increasingly dry fibre tapes. However, this also depended on the type of fibre. After some trials, a relatively consistent sample height of around $3 \mathrm{~mm}$ could be achieved for the lay-up of ten basalt fibre tapes. For a $3 \mathrm{~mm}$ sample, a fibre volume content of about $30 \%$ was calculated. In order to allow for a direct comparison between composite bars with different types of reinforcements, similar sample thickness and fibre content were desired. A calculation of the fibre volume content of a composite bar with a nominal thickness of $3 \mathrm{~mm}$ and ten carbon and alumina fibre tapes, respectively, gave similar fibre volume contents around $30 \%$. However, in practice the thickness of the bars varied with the type of fibre used. Carbon fibre reinforced bars generally tended to be somewhat thinner whereas alumina fibre reinforced bars were usually slightly thicker than $3 \mathrm{~mm}$. Hence, the actual fibre contents also varied to some degree, typically between $25-35$ vol.- $\%$.

The fibre volume content was calculated for each individual composite bar from the ratio of fibre volume to composite bar volume: $\mathrm{V}_{\text {fibre }} / \mathrm{V}_{\text {composite. }}$ The fibre volume was calculated from the measured weight of a single $115 \mathrm{~mm}$ (the length of the composite 
bar) long fibre tape multiplied by the number of fibre layers and divided by the known fibre density. The volume of the composite bar was simply calculated from its width, height and length.

\subsubsection{Nomenclature for composite samples}

Standard composite samples are labelled in the following way: matrix composition (M1 or M5), fibre type (B, C and N610 for basalt, carbon and Nextel 610 alumina fibres, respectively) and serial number (in order of fabrication: $1-5$ ). The letters DS are added behind the label of the fibre type to indicate the use of desized fibres. For example, the label M1-N610-DS-4 represents sample number four of a standard composite bar made from desized alumina fibres and M1 matrix composition. As mentioned before, only three composite samples could be fabricated from each batch of geopolymer binder. Therefore, sample four is in fact the first sample made from a second batch of geopolymer mixture.

\subsection{Heat treatment}

Selected samples were heat treated to investigate the microstructural evolution of the geopolymer matrices and the effect of temperature on the interaction at the fibrematrix interface. An electric chamber furnace (Carbolite 1300) was used at varying temperatures between 500 and $1100^{\circ} \mathrm{C}$ in air. In all cases, a slow ramping programme with a heating rate of $0.5^{\circ} \mathrm{C} / \mathrm{min}$ and annealing steps at $40,60,80$ and $105^{\circ} \mathrm{C}$ was employed to reduce early cracking of bulk specimens due to rapid drying. Subsequently, the heating rate was increased to $2^{\circ} \mathrm{C} / \mathrm{min}$ and $5^{\circ} \mathrm{C} / \mathrm{min}$ up to $200^{\circ} \mathrm{C}$ and above, respectively. The annealing time at the target temperature was 60 minutes in all cases. A schematic drawing of the heating programme is presented in Figure 3.6. It should be noted that this heating programme was not optimised for any particular purpose. All samples were allowed to dry for a minimum of seven days at ambient conditions before firing. 


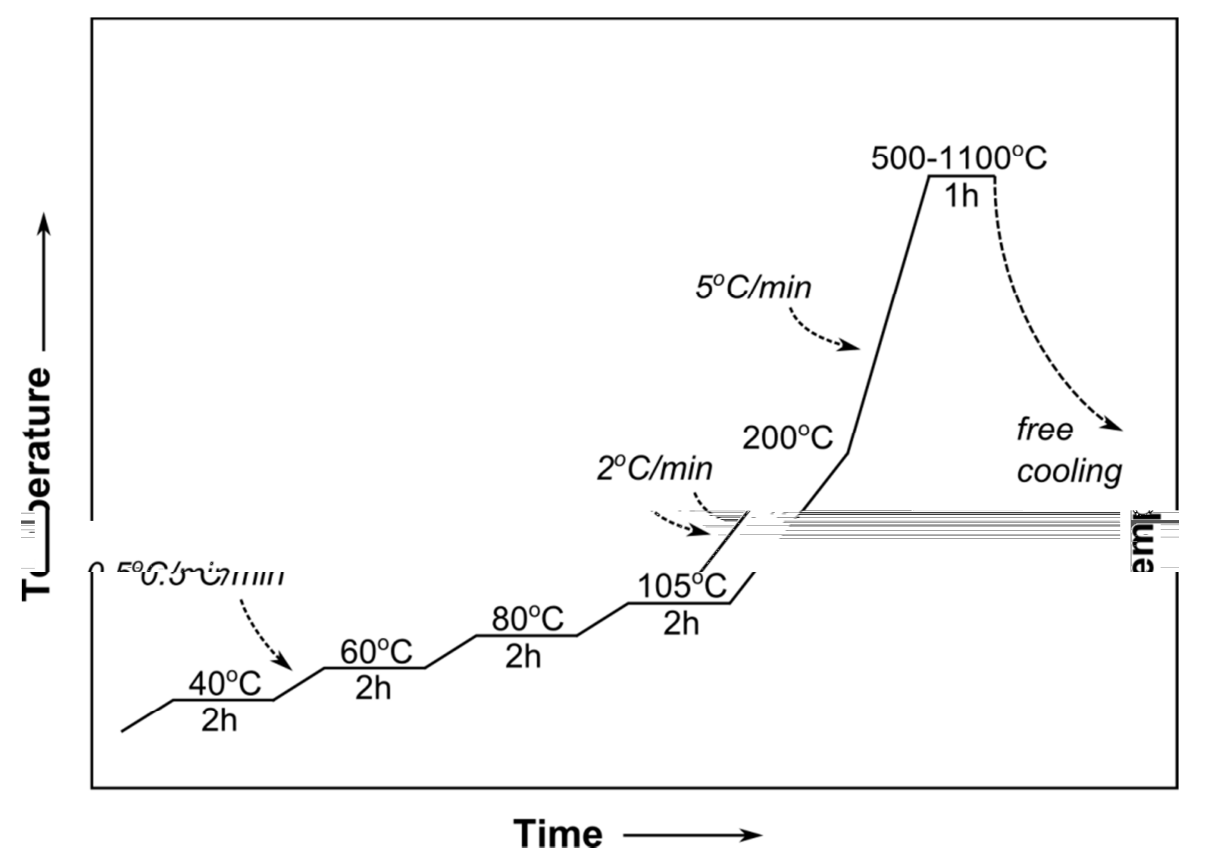

Figure 3.6: Schematic of standard heating programme showing heating rates and annealing steps

\subsection{Ion exchange experiments}

The general feasibility of ion exchange in bulk geopolymer samples was investigated as part of a small side project with the objective to investigate the use of geopolymers and geopolymer composites as potential precursors for the fabrication of ceramics and ceramic composites, respectively. The exchange of the charge balancing cation in the geopolymer matrix could allow some influence on the phase formation at higher temperatures. In particular the exchange of the sodium cation by $\mathrm{NH}_{4}{ }^{+}$and the potential transformation of the ion-exchanged geopolymer matrix into mullite bear considerable potential. The successful ion exchange of ground $\mathrm{Na}$ geopolymer powders by $\mathrm{NH}_{4}^{+}$(and other types of cations) has been described previously by several authors [100-102]. These approaches were used as a general guideline for the ion exchange experiments in the present study.

M1 bar pieces from the flexural testing and cut M1-N610 composite pieces were used as samples for the ion exchange experiments. All bar pieces were roughly $30-$ $40 \mathrm{~mm}$ long and weighed around $2-4 \mathrm{~g}$. Ground M1-powder was also tested as a 
control. All specimens were placed in 0.5 litre plastic bottles with a $0.5 \mathrm{M}$ solution of ammonium chloride $\mathrm{NH}_{4} \mathrm{Cl}$ in a ratio of $100 \mathrm{ml}$ solution per gram of sample. The bottles were subsequently placed on a roller mixer to mix for $24 \mathrm{~h}$. After $24 \mathrm{~h}$, the solution was replaced and mixed again for the same period of time. The samples were then washed with distilled water and dried at room temperature. After drying, specimens were fired at 1000 and $1100^{\circ} \mathrm{C}$, respectively, according to the standard heating programme shown in Figure 3.6. Specimens were examined by SEM, EDS and XRD analyses.

\subsection{Characterisation methods}

\subsubsection{Mechanical properties}

Several methods were used to characterise the mechanical properties of both the unreinforced geopolymer matrix and composite specimens. Unreinforced specimens were tested under tensile, compressive and flexural loading. Flexure and shear tests were conducted on composite specimens. The relevant test methods are described below. Unless stated otherwise, all samples were tested after the standard curing cycle and seven days drying under ambient conditions.

\section{Splitting-tensile strength}

A splitting-tensile strength test, also referred to as Brazil test, was used as a simple and inexpensive method to determine the tensile strength of the unreinforced geopolymer matrix. Similar test methods have been described for example in ASTM C-496 and ASTM D-3967 for concrete and rock core specimens, respectively. These standards were used as a guideline for the present work. The splitting-tensile strength test is a diametral compression test, i.e. a compressive load is applied across the diameter of a cylindrical specimen between two flat platens. This loading configuration induces, besides considerable compressive stresses, tensile stresses along the plane of the applied load [ASTM C-496]. Because geopolymers, like other ceramic materials, show higher compressive than tensile strength, tensile failure 
should occur before compressive failure. The splitting-tensile strength $\sigma_{t}$ can be calculated using equation 3.1:

$$
\sigma_{t}=\frac{2 P_{\max }}{\pi d t}
$$

$$
\begin{aligned}
& \sigma_{\mathrm{t}}=\text { splitting-tensile strength }(\mathrm{MPa}) \\
& \mathrm{P}_{\max }=\text { maximum applied load }(\mathrm{N}) \\
& \mathrm{d}=\text { diameter of the specimen }(\mathrm{mm}) \\
& \mathrm{t}
\end{aligned}
$$

The splitting-tensile strength was determined on $30 \mathrm{~mm}$ diameter pellet specimens with a typical thickness of $10-20 \mathrm{~mm}$. The cured and dried specimens were subsequently mechanically machined to obtain parallel surfaces. An INSTRON 1122 universal testing machine with a $5 \mathrm{kN}$ load cell was used. Specimens were loaded with a constant displacement rate of $2 \mathrm{~mm} / \mathrm{min}$. Thin cardboard strips were placed between the specimens and the machine loading platens to avoid high stress concentration. The load was electronically recorded. The displacement of the specimen was not monitored as this test method is generally not suitable for the determination of the elastic modulus.

\section{Compressive strength}

The compressive strength of the unreinforced geopolymer matrix was determined following ASTM D-695. The compression tests were conducted on small cylinder specimens of $10 \mathrm{~mm}$ diameter and a typical height of $\sim 30 \mathrm{~mm}$. The end surfaces of all specimens were ground plane-parallel prior to testing. The compressive strength can be obtained by dividing the maximum applied load during the test by the original minimum cross-sectional area according to equation 3.2 :

$$
\sigma_{c}=\frac{P_{\max }}{\pi r^{2}}
$$

$$
\begin{aligned}
& \sigma_{\mathrm{c}}=\text { compressive strength }(\mathrm{MPa}) \\
& \mathrm{P}_{\max }=\text { maximum applied load }(\mathrm{N}) \\
& \mathrm{r}
\end{aligned}
$$


All tests were carried out on an INSTRON 1126 universal testing machine with a maximum load capacity of $250 \mathrm{kN}$. A constant displacement rate of $2 \mathrm{~mm} / \mathrm{min}$ was applied. Thin cardboard strips were placed between the specimens and the machine loading platens to avoid high stress concentration. The maximum load was recorded manually. Due to the lack of a suitable displacement recording device for this instrument, the elastic modulus could not be determined in these tests.

\section{Flexural strength}

A three-point bend test was used to determine the flexural strength, strain and elastic modulus of unreinforced geopolymer bar specimens and composite bars. ASTM C-1161, ASTM C-1341 and ASTM D-790 were used as general guidelines for the bending tests. The same test setup was used for unreinforced and composite specimens. All tests were carried out using an INSTRON 1122 universal testing machine equipped with a $500 \mathrm{~N}$ load cell. The testing was displacement controlled with a constant rate of $2 \mathrm{~mm} / \mathrm{min}$. The displacement of the specimen was measured with a LVDT (Linear Variable Differential Transformer) attached to the loading frame of the instrument. The measurement of the displacement at the mid-span of the specimen would have been preferred for a more accurate measurement. However, such a setup was not feasible with the available equipment. Therefore, some error has to be accepted for these measurements. Nevertheless, the loads that were applied during the tests were relatively low and any possible extraneous deformations, which may be an issue at higher loads, should be minimal. Since the same error applies to all specimens tested in this study, this does not affect the comparability between specimens. The load and the LVDT displacement were electronically recorded. The support and loading pins of the testing jig had a diameter of $5 \mathrm{~mm}$. The standard support span for both unreinforced and composite specimens was $100 \mathrm{~mm}$. However, shorter spans were also applied in some cases to evaluate the influence of the spanto-depth ratio on the measured properties.

The fabrication of unreinforced and composite bar specimens was described in chapter 3.3 and 3.4.3, respectively. All specimens were tested in the orientation as they were fabricated, i.e. the bottom surface of the as-fabricated specimen was also 
the tensile surface during testing. For the most part, all specimens were tested in their as-fabricated state. However, some additional preparation was necessary. The unreinforced bars had to be lightly sanded by hand along the side surfaces in order to obtain clean edges. The edges on the tensile side were chamfered. The tensile and compression surfaces remained otherwise unchanged compared to the as-fabricated specimen. The standard unreinforced bars measured approximately $6 \times 10 \times 120 \mathrm{~mm}$. Following the standard test on the $100 \mathrm{~mm}$ support span, the two fractured bar pieces of each specimen were tested again on a $40 \mathrm{~mm}$ span. The composite specimens required some more preparation. Although the fabrication method allowed the fabrication of relatively even composite bars, some surface irregularities could not be avoided and the side and compressive surfaces of most specimens had to be sanded to some degree. However, the original bottom/tensile surface of the specimen was not altered. All sanding was conducted dry and by hand using grit \#320 SiC paper. The dimensions of the standard composite bars were approximately $3 \times 10 \times 115$ $\mathrm{mm}$. For the standard $100 \mathrm{~mm}$ testing span, this corresponded to a span-to-depth ratio of roughly $32: 1$. For comparative purposes, some composite bars were cut in half and tested on a $50 \mathrm{~mm}$ span, corresponding to a s/d ratio of approximately 16:1. A spanto-depth ratio of 16:1 is the minimum ratio suggested by both ASTM C-1341 and ASTM D-790 for three-point bend tests in order to minimise shear stresses in the specimen and induce tensile failure. However, higher s/d ratios are suggested for materials with low shear strength.

The flexural properties of the unreinforced and composite specimens can be calculated in similar fashion. These calculations are based on elastic beam theory assuming homogeneous and linear elastic behaviour of the tested material. Whereas this applies directly to the unreinforced beams the same assumptions have to be made for composite beams. According to ASTM C-1341 these assumptions are largely valid for composites with the principal fibre direction along the axis of the beam as long as the deflections are relatively small (extreme fibre strain $<5 \%$ ).

In general terms, the stress $\sigma$, in the elastic region, is linearly proportional to the strain $\varepsilon$ according to: 


$$
\sigma=E \varepsilon
$$

where $\mathrm{E}$ is a constant factor referred to as the modulus of elasticity. Under threepoint flexural loading, the maximum stress occurs in the outer surface / extreme fibre at the central loading point. It can be shown that the flexural stress of a rectangular beam can be calculated by the following equation:

$$
\sigma_{f}=\frac{3 P L}{2 b h^{2}}
$$

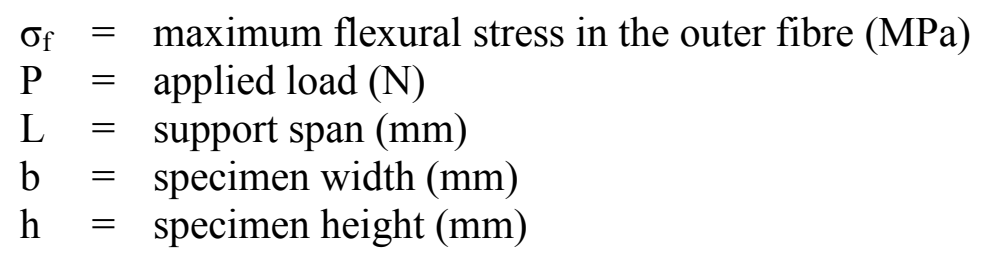

The ultimate flexural strength, as reported in this work, is calculated at the point of maximum applied load. Using the measured deflection, the flexural strain $\varepsilon$ can be calculated according to equation 3.5 :

$$
\begin{gathered}
\varepsilon_{f}=\frac{6 \delta h}{L^{2}} \\
\varepsilon_{\mathrm{f}}=\text { flexural strain }(\mathrm{mm} / \mathrm{mm}) \\
\delta=\text { deflection }(\mathrm{mm})
\end{gathered}
$$

From equation (3.3), the modulus of elasticity $\mathrm{E}$ is the quotient of stress to corresponding strain and equals the slope of the tangent to the steepest initial linear section of the load-deflection curve. Combining equations (3.3), (3.4) and (3.5), the flexural elastic modulus can be calculated by

$$
E_{f}=\frac{m L^{3}}{4 b h^{3}}
$$




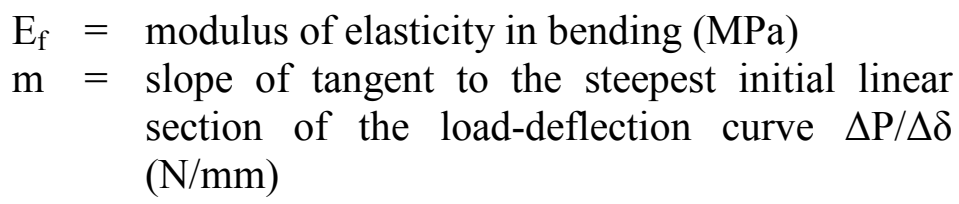

In practice, some curves showed a non-linear initial region, also referred to as the "toe" region. This toe is not represent the material property and toe correction was carried out where necessary (see e.g. ASTM D-790).

\section{Short beam shear strength}

Short beam tests were carried out to obtain some indication of the interlaminar shear strength of selected composite bars. ASTM D-2344 was used as a guideline for these tests. Essentially, the short beam shear test resembles a three-point bend test on a very small load span. Therefore, the same testing equipment and general parameters as described for the three-point bend tests were applied. By reducing the load span the shear stress in the specimen can be maximised. As a result, shear failure of the specimen with a crack along the mid-plane is ideally introduced as opposed to tensile failure which is typically observed for longer load spans. The clear observation of mid-plane interlaminar failure is an essential requirement for the validity of this test. The maximum interlaminar shear stress $\tau_{\max }$ is proportional to the maximum load $\mathrm{P}_{\max }$ and can be calculated using the following equation:

$$
\tau_{\max }=\frac{3 P_{\max }}{4 b h}
$$

Three different support spans of 10, 15 and $20 \mathrm{~mm}$ were tried on the various specimens. Depending on the support span, standard composite bars were cut into four or five equally long pieces with a diamond saw and IPA as a coolant. Due to a limited amount of alumina fibres only basalt and carbon composites were tested.

\subsubsection{Electron microscopy}

Electron microscopy was used to analyse the microstructure of the various geopolymer matrices and composites, in particular the interaction at the fibre-matrix interface. Polished and fractured surfaces were both investigated. Most of the results 
presented in this work were obtained using a JEOL JSM 6500F scanning electron microscope (SEM), equipped with an energy-dispersive x-ray spectrometer (EDS, JEOL Ex23000BU). A JEOL JSM 5300LV SEM was also used in some cases during the early stages of this work. However, due to its minor relevance, this section will only focus on the work with the afore-mentioned instrument. Standard secondary electron imaging and backscattered imaging modes were equally employed throughout this study. Both imaging modes had advantages depending on the sample type and the particular aspect under investigation. The respective imaging mode is indicated by the following abbreviations at the bottom of each micrograph: SEI (secondary electron) and COMPO (composition mode in backscattered electron imaging). Standard EDS imaging conditions (accelerating voltage: $15 \mathrm{kV}$; working distance: $10 \mathrm{~mm}$ ) were applied in all cases. Elemental mapping and line scan profiles were used as the main EDS analysis techniques. The line scan profiles were particularly useful for examination of the concentration gradients of relevant elements across the fibre-matrix interface. The line scans provide a qualitative profile of the relative elemental concentration of each element along the scanned line. In order to directly compare the concentration trends of the various elements irrespective of their abundance, all line scan profiles presented in this study were normalised in regards to the maximum intensity of each element.

The microstructure of unreinforced geopolymer matrices was investigated on fracture surfaces of bar specimens after flexural testing. The bars were cut into roughly $4 \mathrm{~mm}$ thin slices using a diamond saw. Isopropanol (IPA) was used as the cooling agent for all cutting processes. Randomly fractured pieces were used for the examination of heat treated geopolymers. The flexural strength testing of fibre reinforced bars did generally not result in a complete failure of the composite samples. Therefore, fracture surfaces of these samples had to be obtained by other methods. Consequently, none of these fracture surfaces represent the true fracture surface obtained from the mechanical testing. Fibres with long pull-out length or nonfractured fibres had to be cut with a razorblade to a practical length for the examination in the SEM. Polished surfaces were obtained by vacuum assisted moulding of respective samples in epoxy resin and subsequent manual polishing 
(STRUERS DP 10). Following an initial grinding step with grit \#320 SiC paper to plane the surface, all samples were polished on diamond lapping films (ALLIED High Tech Products Inc., USA) in steps of 30, 15, 9, 3 and $1 \mu \mathrm{m}$. Water had to be used as a lubricant for all polishing processes due to restrictions of the setup of the polishing equipment. All samples for SEM analysis were mounted on adequate aluminium stubs of approximately $10 \mathrm{~mm}$ diameter with adhesive carbon tape. The prepared samples were subsequently coated with carbon (QUORUM Q150T sputter coater, UK) and stored in a desiccator with an attached rotary pump. Due to the extremely fine porosity of the geopolymer matrix, all samples were desiccated for a minimum of three days. The pre-desiccation of the samples was necessary in order to achieve the required high vacuum in the loaded SEM sample chamber.

\subsubsection{X-ray powder diffraction}

X-ray powder diffraction (XRD) was used to identify crystalline phase formation in selected geopolymer and composite samples. A PANalytical X'Pert PRO MPD diffractometer with a PIXcel detector and monochromatic $\mathrm{Cu} K \alpha_{1}$ radiation $(\lambda=$ $1.5418 \AA$ ) was used for all measurements. Standard instrument generator settings (excitation voltage: $45 \mathrm{kV}$; current: $40 \mathrm{~mA}$ ) were applied. Diffraction patterns were recorded between 8 and $80^{\circ} 2 \theta$ with a step size of 0.02 degrees and 500 counts per step. A spinning stage with $2 \pi / 16 \mathrm{~s}$ was used in all cases. All samples were finely ground with a mortar and pestle. The recorded diffractograms were analysed with the software PANalytical X'Pert HighScore. Due to the predominantly amorphous nature of geopolymers (and some fibres) most diffraction patterns showed significant background features. Although this was taken into account for the phase matching, none of the diffraction patterns presented in this work were subjected to any background correction.

\subsubsection{Nuclear magnetic resonance}

${ }^{29} \mathrm{Si}$ and ${ }^{27} \mathrm{Al}$ magic angle spinning nuclear magnetic resonance spectroscopy (MAS NMR) were used to determine the atomic coordination states of silicon and aluminium in the five fully cured geopolymer matrix compositions M1 - M5. All samples were finely ground with a mortar and pestle and dried prior to analysis. The 
measurements were carried out externally by Industrial Research Limited (IRL, Lower Hutt, New Zealand). Details on the equipment and applied instrument settings are given in Table 3.5.

Table 3.5: Instrument settings for ${ }^{29} \mathrm{Si}$ and ${ }^{27} \mathrm{Al}$ MAS NMR

\begin{tabular}{lcc}
\hline & ${ }^{29}$ Si MAS NMR & ${ }^{27}$ Al MAS NMR \\
\hline Spectrometer & \multicolumn{1}{c}{ BRUKER Avance 500} \\
Doty MAS probe & $5 \mathrm{~mm}$ & $4 \mathrm{~mm}$ \\
Rotor speed & $\sim 6 \mathrm{kHz}$ & $10-12 \mathrm{kHz}$ \\
Frequency & $99.296 \mathrm{MHz}$ & $130.224 \mathrm{MHz}$ \\
Pulse & $6 \mu \mathrm{s}$ & $1 \mu \mathrm{s}$ \\
Delay & $30 \mathrm{~s}$ & $1 \mathrm{~s}$ \\
Reference & tetramethylsilane & $\mathrm{Al}\left(\mathrm{H}_{2} \mathrm{O}\right)_{6}{ }^{3+}$ \\
& $(\mathrm{TMS})$ & \\
\hline
\end{tabular}

\subsubsection{Thermal analysis - TGA/DSC}

A combined TGA/DSC analysis was used to obtain some information on the thermal behaviour of the geopolymer matrix composition M1. The dried sample was finely ground with a mortar and pestle prior to analysis. The measurement was carried out externally by Industrial Research Limited (IRL, Lower Hutt, New Zealand) using a TA Instruments, SDT Q600 simultaneous TGA/DSC analyser. The thermal behaviour was analysed up to $1100^{\circ} \mathrm{C}$ with a heating rate of $10^{\circ} \mathrm{C} / \mathrm{min}$ and an air gas flow rate of $50 \mathrm{ml} / \mathrm{min}$. 


\title{
Chapter 4
}

\section{Characterisation of geopolymer matrices}

\begin{abstract}
After initial tests, four different geopolymer mixtures (M1 - M4) were selected for further characterisation in order to identify the most suitable matrix composition for the composite fabrication. An optimised matrix composition (M5) was introduced at a much later stage of the project and was therefore not part of some of the earlier investigations. The main goal of this part of the study was to determine the basic mechanical and microstructural behaviour and properties of the different matrices. However, the focus was on the main matrix composition M1. The findings are presented and discussed in the following chapters.
\end{abstract}

\subsection{Fabrication process and initial investigations}

\subsubsection{Mixing, viscosity and use-time of geopolymer binders}

Early small scale mixtures were prepared by hand mixing. This process generally resulted in the formation of an initially stiff, dry and lumpy mixture that gradually smoothened, eventually turning into a viscous slurry after continued mixing with a spatula for about 10 minutes. All of these mixtures were pourable, showing viscosities between what can be described as medium viscous fluids to thin pastes. Vibrating of the slurries supported their flowability. Compositions M2 and M4 
showed the lowest viscosity and could be cast into moulds relatively easily. A somewhat higher viscosity was observed for composition M1. M3 showed the highest viscosity of all compositions despite the highest nominal $\mathrm{H}_{2} \mathrm{O} / \mathrm{Na}_{2} \mathrm{O}$ ratio.

To improve the reproducibility of the mixing process as well as the homogeneity of the geopolymer binder, in particular for larger amounts, a mechanical mixing process was employed for all subsequent experiments. The advantages of the mechanical mixing process were immediately noticeable: by slowly adding the metahalloysite into the stirring alkali solution, the formation of the stiff and lumpy pre-paste, as described above for hand-mixed binders, could be avoided and much smoother and seemingly more homogeneous mixtures could be achieved after shorter mixing times. The mechanical mixing also resulted in a considerable decrease in the viscosity of all geopolymer binders. This apparent reduction in viscosity can be attributed to a much better homogenisation of the binder during the mechanical mixing process compared to the hand mixing. The reduced viscosity worked in favour of the cast-ability and therefore quality of the unreinforced geopolymer specimens as well as allowing better impregnation of fibre bundles. Viscosity measurements were attempted at the German Aerospace Centre in Cologne, Germany, but had to be abandoned after initial tests due to corrosive attack of the viscometer. The acquisition of quantitative data was not pursued any further.

The pot-life of the different compositions typically ranged between 15 - 30 minutes. After this time, the onset of the polymerisation reaction caused a rapid increase in viscosity of the slurry, making it unusable for the desired purposes. Whereas this timeframe was sufficient to mould unreinforced specimens, it only allowed the fabrication of one composite sample per batch of geopolymer mixture. To extend the effective use-time of the geopolymer binder, continuous stirring of the mixture during the fabrication of composite samples was considered as an option. The continuous mixing proved successful in retarding the polymerisation and setting reaction of the geopolymer binder and the effective use-time could be extended up to 45-60 minutes. This allowed the fabrication of three composite samples per batch of geopolymer mixture. However, the extended pot-life came at the cost of a decreased 
use-time of the geopolymer binder after the mixing process. This effect became increasingly obvious for mixing times greater than $\sim 30$ minutes. After a mixing period of $45-60$ minutes the rapid setting of the binder reduced the processing time to no more than a few minutes. This indicates that the delay of the onset of the geopolymerisation reaction in the early stage leads to an increasingly accelerated reaction following the end of the mixing process.

During the course of the continuous mixing process a slight change of the viscosity with increasing mixing time was also noted. Compared to the standard five minutes homogenisation time, the viscosity appeared to decrease slightly for longer mixing times, reaching a minimum viscosity after around $10-15$ minutes before slowly increasing again. This decrease in viscosity is most likely a result of better homogenisation and a higher degree of reactivity over mixing time due to the destruction of some agglomerates that may be present in the geopolymer binder and better dissolution of remaining metahalloysite particles. However, the mechanism responsible for this reduction in viscosity competes against the onset of the geopolymerisation reaction and the setting of the geopolymer which result in a gradual increase in viscosity at longer mixing times. Thus, the desire to achieve better homogeneity of the binder by extending the mixing time has to be balanced with the practical requirement of the use-time of the geopolymer binder after mixing. While $~ 15$ minutes seemed to be the optimal mixing time for the particular compositions and mixing process used in this study in regards to the degree of homogeneity and viscosity, the mixing time was too long for the required purpose. Therefore, a compromise had to be made and 5 minutes were set as the standard homogenisation time of all geopolymer binders.

\subsubsection{Optimisation of the fabrication process}

The readily mixed binder could contain considerable amounts of entrapped air, leaving large pores in the hardened solid and along the bottom edges. These defects have significant effect on the mechanical strength of the material. Therefore, several methods to eliminate large entrapped air bubbles in the cast specimen were investigated in preliminary tests, including mixing under vacuum and low frequency 
vibration of the homogenised mixture. For the vacuum mixing the mixing container was fitted with a lid that connected to a vacuum pump after all metahalloysite was added to the mixture. Although this method showed some potential to reduce the enclosed air volume, having to dis- and reassemble the mixer setup proved time consuming and overall impractical. Therefore, this option was not considered any further. Vibrating of the geopolymer binder after mixing on a vibration table (sideward motion), on the other hand, was found to be largely ineffective. However, tapping the mixing container repeatedly on a solid surface in a vertical motion caused most of the entrapped air bubbles to surface and resulted in a significantly reduced air volume in the binder. Further improvement of the sample quality could be achieved by optimizing the fabrication process, i.e. controlling the actual casting process and reducing the thickness of the casting stream. The thinner casting stream had two principal advantages: (i) entrapped air bubbles were more likely to burst in the thinner stream and (ii) the chance of entrapping air along the edges of the mould during the casting of the sample was reduced. The thickness of the casting stream could be altered by adjusting the pouring height. A height of about $30-40 \mathrm{~cm}$ was found to be optimal for this study. With regards to improvements of the casting process itself it was favourable to fill all moulds on a vibration table. The vibration helped the binder to easily spread in the moulds reducing defects along the edges of the cured specimens. Also, a slow but constant pouring of the liquid binder helped to improve sample quality. This was particularly important for the fabrication of narrow cylinders for compression testing. A fine casting stream was required to cast these samples and the best results were obtained when all five samples per row were cast in a single step under a continuous stream flow.

\subsubsection{Drying process}

The initial characterisation of the four original matrix compositions (M1 - M4) was carried out using pellet specimens. For the evaluation of the mechanical properties of these samples, a splitting-tensile strength test was used. The mechanical properties will be addressed in chapter 4.2. Here, only some general aspects of the drying behaviour of the pellet specimens shall be described. 
As mentioned before, all matrix compositions investigated in this study produced viable solid geopolymers after the standard curing process. The developed hardness of all compositions after curing was good, and except for composition M3, which appeared somewhat softer than other mixtures, all compositions resisted a simple scratch test with a spatula. The demoulded pellets (as well as all other samples) were typically dried under ambient conditions on a lab bench. Some small variations (approximately $20 \pm 2 \%$ ) were observed between different batches of composition M1, most likely due to temperature and humidity fluctuations of the lab environment. The weightloss of the different compositions also varied slightly. The typical drying behaviour of the pellet specimens of all four geopolymer compositions is shown in Figure 4.1. A level of constant weight was usually reached after around three days.

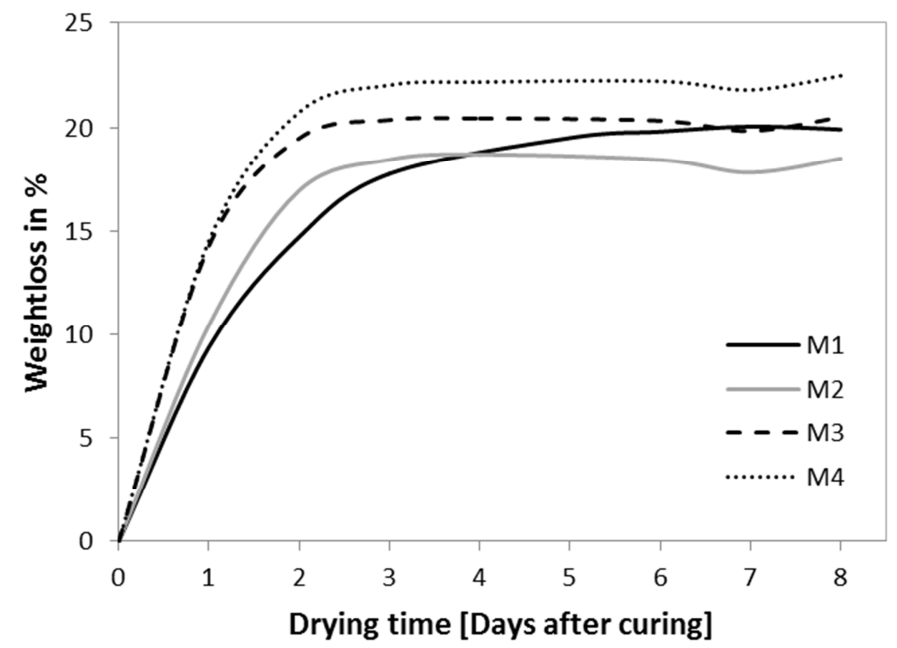

Figure 4.1: Typical weightloss of pellet specimens of compositions M1 - M4 during the drying process under ambient conditions

No noticeable shrinkage of the pellets could be measured over the course of the drying process. However, the formation of drying cracks was a major issue for pellet specimens. The other unreinforced sample types, i.e. cylinder and bar specimens, on the other hand, were not affected by drying cracks. The cracking of pellet specimens was only observed after a number of samples had already been successfully fabricated and mechanically tested (see chapter 4.2.2 for more detail). Although some cracking was observed among these initial samples, the majority of these samples appeared reasonably strong and crack free. In comparison between the four 
different geopolymer compositions, M2 appeared to have the highest and M3 the lowest tendency to crack. These initial mixtures were mostly prepared by handmixing but some batches of supposedly crack free pellets could also be fabricated from mechanically mixed geopolymer binder. At some stage throughout the experiments, a whole batch of samples was found to be subjected to a number of macroscopically visible cracks. Surprisingly, however, most of these cracks seemed to have disappeared the following day. This phenomenon was subsequently repeatedly observed. It was noticed that the initial crack formation generally occurred within the first $24-48$ hours of the drying process. The subsequent disappearance of some fine cracks, however, only occurred on a macroscopic level. Wiping the sample surfaces with isopropanol alcohol (IPA) was found to be a fast and simple method to reveal these cracks without the need of time-consuming microscopic analysis. In fact, wiping the sample surfaces with IPA revealed not only the disappeared cracks but for most pellets also a considerable number of previously unnoticed microcracks, see Figure 4.2. Therefore, it is arguable if the earlier samples were truly crack free or also included microcracks. The disappearance of cracks in the specimens is most likely caused by minimal shrinkage in the pellet specimens, inducing a closing force on the present cracks.
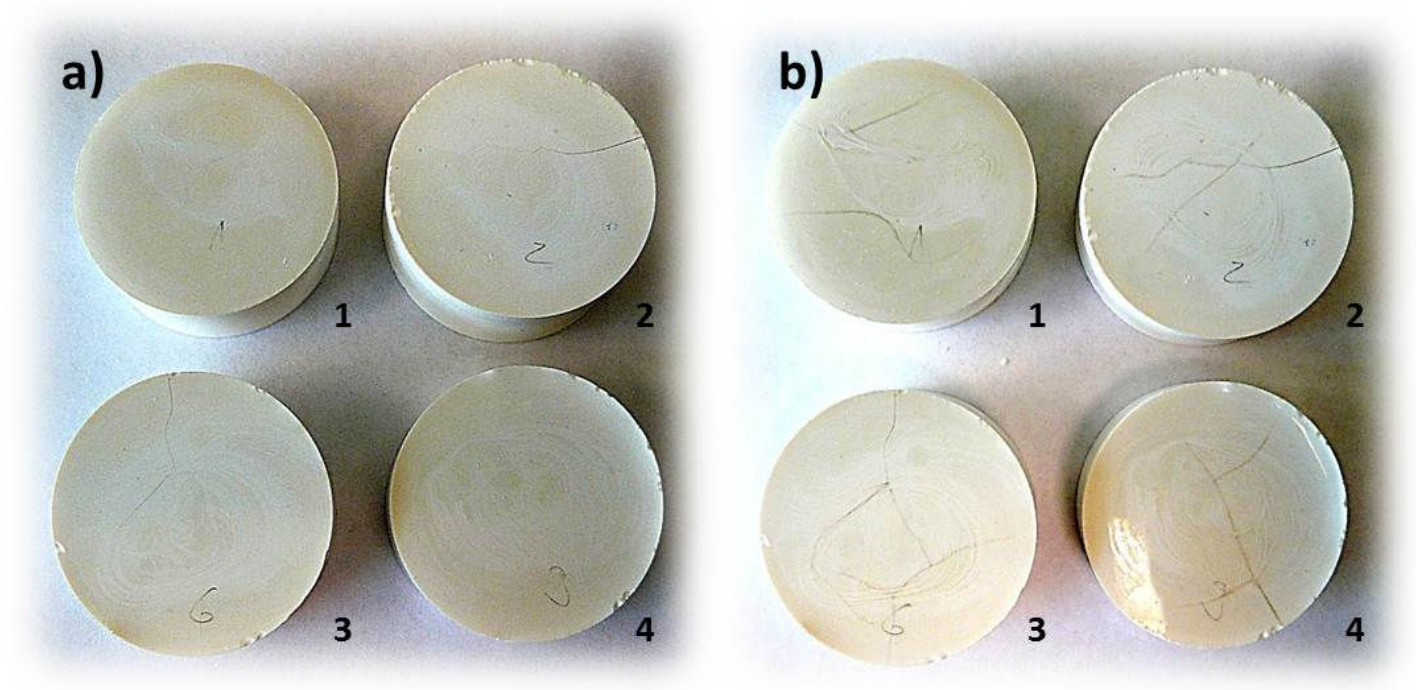

Figure 4.2: Visibility of micro- and macrocracks in M1 pellets before (a) and after (b) wiping the sample surfaces with IPA. The appearance of several microcracks in the seemingly crack free samples 1 and 4 are clearly visible 
Subsequently, considerable efforts were made to control and prevent the cracking of pellets including the drying under different controlled humidities and the addition of small amounts of glycerol as reported by Barbosa et al. [89]. However, all attempts proved largely unsuccessful and crack free pellets were only obtained in occasional instances. Since sample cracking was only an issue for the pellet specimens, the fabrication of this sample type was eventually abandoned.

It was initially believed that the cracking problem may be a result of the particular chemical compositions of the geopolymers or the particular matrix system. However, since the formation of drying cracks was not observed in bar and cylinder specimens of the same compositions, the chemical composition is not considered the main cause for the cracking. Nevertheless, the fact that some compositions appeared more prone to cracking than others indicates that the chemical composition, in particular the alkali content, may have some effect. Another possible explanation may be related to the overall sample size. This is based on the fact that only the larger pellet specimens were subject to cracking. Also, the surface-to-volume ratio of the specimens might have an effect. Both pellet and bar specimens had similar volumes but the surface-tovolume ratio of the bar specimens was nearly double compared to the pellet specimens. This may unfavourably affect the water evaporation during the drying process, resulting in cracks. Although the development of drying cracks in larger specimens may be overcome technologically by a more carefully controlled drying process, the present results suggest that the fabrication of crack-free metakaolin geopolymers with a cross section of more than roughly $10 \times 10 \mathrm{~mm}$ becomes increasingly problematic. However,

\subsection{Mechanical and physical properties}

The mechanical properties of the various geopolymer compositions were characterised by means of splitting-tensile, compression and flexural test methods as described in chapter 3.7.1. Unless stated otherwise, all samples were tested after a standard drying time of seven days. The bulk density was measured on cylinder 
specimens prior to compressive strength testing. The results of the respective investigations are summarised in the following sections and are critically discussed at the end of this chapter.

\subsubsection{Density}

The bulk density of the different mixtures was determined from the volume and weight of the specimens on small cylinder specimens prior to compressive strength testing. The densities of the five compositions were very similar and ranged between approximately 1.3 to $1.5 \mathrm{~g} / \mathrm{cm}^{3}$ with M3 and M5 showing the lowest and highest density, respectively, see Table 4.1.

Table 4.1: Bulk densities of compositions M1 - M5 measured on small cylinder specimens

\begin{tabular}{lccccc}
\hline & M1 & M2 & M3 & M4 & M5 \\
\hline Density $\left[\mathbf{g} / \mathbf{c m}^{\mathbf{3}}\right]$ & 1.43 & 1.37 & 1.29 & 1.30 & 1.44 \\
\hline
\end{tabular}

\subsubsection{Splitting-tensile strength}

The splitting-tensile test was the method of choice for the initial evaluation of the strength of the various geopolymer compositions. This method was mainly chosen due to the unavailability of suitable moulds to produce samples for any other form of mechanical testing in the early stages of this work but also due to the ease of sample fabrication and the previous application of this test method in the research group. The splitting-tensile test method was also used to study the effect of several other parameters such as curing time, mixing process and sample preparation (machining/grinding) on the strength of the geopolymer matrix M1. However, due to the cracking issues of the pellet specimens (described in chapter 4.1.3), the splittingtensile tests produced largely invalid results. Therefore, this subject shall be summarised only briefly in the following paragraph.

Initial mechanical tests of the four hand-mixed geopolymer compositions M1 - M4 achieved strength values between around $0.5-3 \mathrm{MPa}$, see Figure 4.3. Although the highest overall strength was achieved by M2, composition M1 achieved a higher 
average strength. Due to the large variation of the M2 pellets and the previous observation that the M2 pellets appeared to show a higher tendency to crack during drying, M1 was chosen as the main matrix composition for this study.

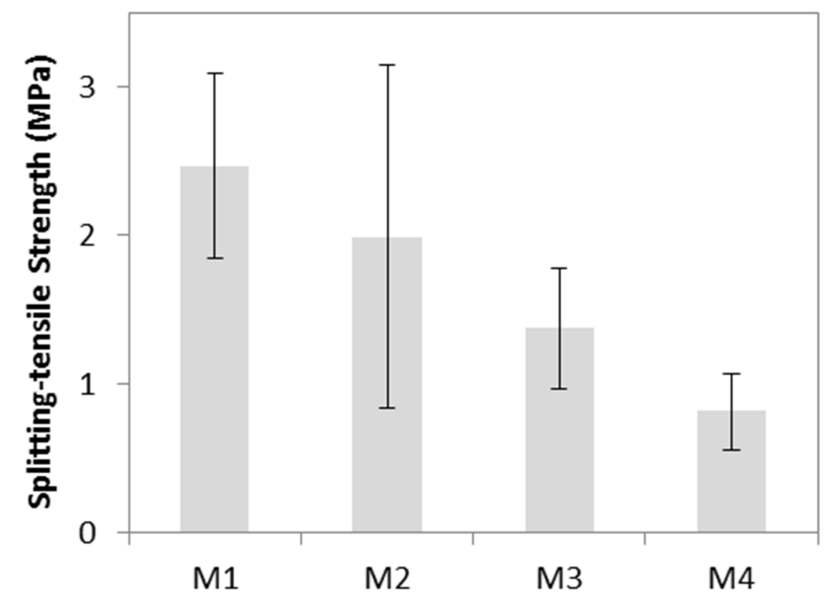

Figure 4.3: Initial evaluation of the mechanical strength of the hand mixed compositions M1-M4 by means of splitting-tensile strength measurements

By improving the general fabrication method and proper machining of the asprepared end surfaces of the pellets prior to testing the average strength of handmixed M1 samples could be improved to $3.8 \mathrm{MPa}$, see Figure 4.4 (M1-2). The application of a mechanical mixing process resulted in a further improvement of the splitting-tensile strength with an average value of $\sim 4.4 \mathrm{MPa}$ measured for each of two consecutive batches and a maximum strength of $6.4 \mathrm{MPa}$. It should be mentioned, that the effect of drying time was somewhat neglected in these early experiments and most of the above results were achieved after a drying time of $10-$ 20 days. Up to this stage, excessive sample cracking did not appear to be an issue and all tested samples were generally considered to be crack free. However, excessive sample cracking became a major issue in subsequent experiments designed to analyse the effects of various parameters on the strength of the geopolymer matrix in some more detail. Despite the apparent presence of cracks in these samples, they still achieved average strength values ranging between 1.6 - 3.2 MPa. Although this strength is appreciable and similar to some of the hand mixed sample series despite the obviously weakened structure of the test samples, these values have to be generally considered invalid. A number of other tests, not displayed in Figure 4.4, 
were performed with regard to overcoming the cracking problem but with limited success. Only one crack free batch consisting of ten samples could subsequently be produced, achieving an average strength of $4.2 \mathrm{MPa}$ after two days of drying, see Figure 4.4 (M1-14).

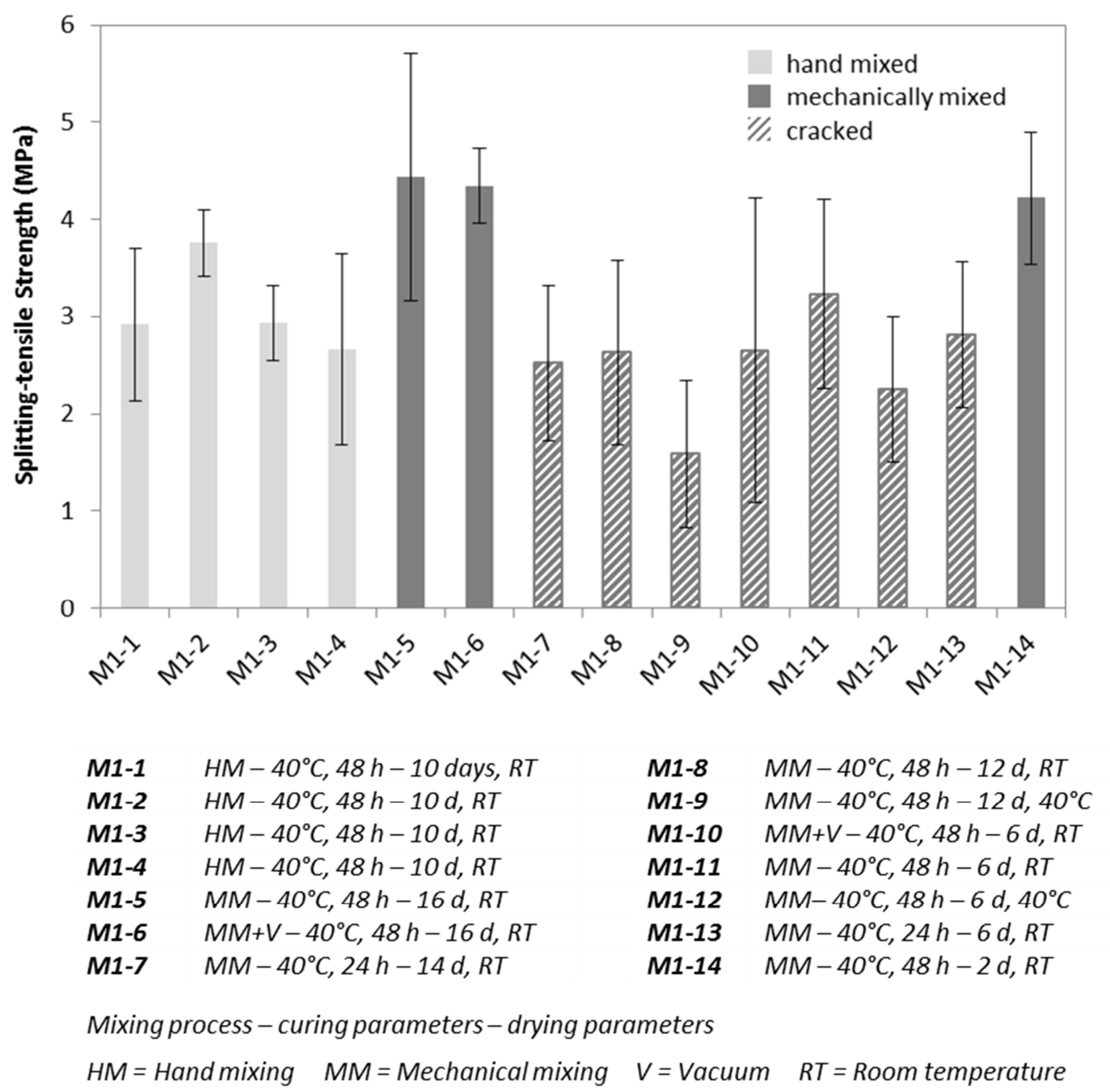

Figure 4.4: Splitting-tensile strengths of selected sample series of composition M1 subjected to different mixing, curing and drying parameters. Several different machining and grinding preparations were tested on the four hand mixed sample batches (not further specified here). All other sample series were mechanically machined in the same way

\subsubsection{Compressive strength}

The compressive strength was measured on small cylinders of approximately $10 \mathrm{~mm}$ diameter and a typical height-to-diameter ratio of 3:1. However, the sample height had no notable effect on the compressive strength and similar strengths were 
obtained for specimens with a 2:1 ratio. Prior to the testing, it was essential for the end surfaces of all specimens to be ground parallel. In most cases, the grinding was carried out using a dry method to avoid any weakening of the material. This was due to the fact that the contact with water during any cutting or grinding process generally caused cracking and, thus, premature failure of most unreinforced specimens. Replacing water with isopropanol alcohol (IPA) as the liquid medium, however, offered a suitable alternative and allowed wet grinding and cutting processes without any unfavourable effect on the strength of the geopolymer matrix. The dry and IPA grinding method were compared on batch M1-c in Table 4.2. Of the total of ten samples of this batch, five samples were prepared according to each of the two different methods. The standard deviation of the compressive strength of batch M1-c was the lowest of all tested sample batches.

Table 4.2: Variability of compressive strength between different batches (including the strongest and weakest) of composition M1 all prepared by the same fabrication process and conditions

\begin{tabular}{cccccc}
\hline & M1-a & M1-b & M1-c & M1-d & M1-e \\
\hline Mean & 78.9 & 31.0 & 76.1 & 35.2 & 57.1 \\
Stdev & 6.5 & 3.4 & 1.8 & 5.5 & 13.9 \\
\hline
\end{tabular}

Several batches of composition M1 were tested in order to obtain a reasonable number of specimens for statistical evaluation. However, the experiments showed poor reproducibility and very large variability of compressive strength between the different batches. The average compressive strength and the respective standard deviation of five different batches of M1, including the two strongest and weakest sample series, are displayed in Table 4.2. Batches M1-a to -c comprised of ten and M1-d and -e of five specimens, respectively. The resulting strength of each batch of samples was at random and generally unpredictable. The significant variation between the different batches of the same material indicates a problem with the fabrication process but since all batches were fabricated according to the same process and from the same materials, there is no obvious explanation for this behaviour. Despite the significant variation between the different sample series, it should be noted that the variability of the compressive strength within each series was reasonably good. A large variation within a set of samples as displayed by M1-e 
was uncommon. Due to the variability of the strength of the different sets of samples, a statistical evaluation was disregarded.

The number of samples tested for matrix compositions M2 to M5 was much smaller and in most cases limited to two sample batches. The two batches of compositions M2 and M3 indicated similar variability as observed for M1. The two batches of composition M5 on the other hand showed very similar compressive strengths. The batches with the highest average compressive strength of each matrix composition are compared in Table 4.3. The sample series with the highest average strengths were chosen based on the assumption that these values are closer to the true strength of each composition and that these results could possibly be reproduced more reliably with better control of the mixing, fabrication and drying processes. Composition M3 showed the lowest compressive strength of all compositions with an average strength of $53 \mathrm{MPa}$. An average strength of $68 \mathrm{MPa}$ was measured for composition M4. Composition M1 and M2 obtained roughly similar strength with 79 MPa for M1. A very high strength was measured for composition M5 with an average compressive strength of about $136 \mathrm{MPa}$ and an overall maximum strength of $145 \mathrm{MPa}$. The second batch of M5 produced similar results with an average of $131 \mathrm{MPa}$.

Table 4.3: Average compressive strength of the strongest batch of all matrix compositions

\begin{tabular}{lccccc}
\hline & M1 & M2 & M3 & M4 & M5 \\
\hline Mean & 78.9 & 74.9 & 52.6 & 68.2 & 135.6 \\
Stdev & 6.5 & 7.4 & 5.5 & 5.5 & 5.8 \\
\hline
\end{tabular}

The effect of the drying time on the compressive strength of composition M1 is presented in Table 4.4. The 28 day strength was only measured on one batch consisting of five samples. Since it is unknown what the strength of the same batch was after seven days of drying and given the strength variation of composition M1 as described before, the effect of the drying time could not be quantified without difficulty. However, the result indicates a considerable increase in compression strength for extended drying times. 
Table 4.4: Influence of drying time on the compressive strength of composition M1

\begin{tabular}{ccc}
\hline & $\mathbf{7}$ days & 28 days \\
\hline Mean & 78.9 & 94.6 \\
Stdev & 6.5 & 13.8 \\
\hline
\end{tabular}

\subsubsection{Flexural strength and modulus}

The flexural properties of compositions M1 - M5 were determined in a three-point bend test using rectangular bars of the approximate size $10 \times 6 \times 120 \mathrm{~mm}$. All bars were tested on a $100 \mathrm{~mm}$ span. The fractured bar pieces were subsequently tested again on a $40 \mathrm{~mm}$ span. The average strength values measured for the 100 and 40 mm spans of each batch (five bars per batch) varied to various degrees. In most cases, the $40 \mathrm{~mm}$ span tests achieved somewhat higher strength. However, some sample batches also showed lower or significantly higher average strengths for the $40 \mathrm{~mm}$ compared to the $100 \mathrm{~mm}$ span. The flexural modulus was only measured in tests on the $100 \mathrm{~mm}$ span. The results for the $100 \mathrm{~mm}$ span tests are presented in Table 4.5 and Table 4.6.

Several batches of composition M1 were tested in order to obtain a significant number of samples and to verify the reproducibility of the results. Three of these batches showed relatively good reproducibility with an average strength of roughly $10 \mathrm{MPa}$, see Table 4.5. The strength of the individual samples for batches M1-2 to M1-4 ranged between approximately $8-13 \mathrm{MPa}$. The average flexural strength of batches M1-2 and M1-4 over the $40 \mathrm{~mm}$ span was slightly higher with 11.2 and 10.6 $\mathrm{MPa}$, respectively, but still within the same range as for the $100 \mathrm{~mm}$ span. However, batch M1-3 showed a considerably higher average strength of $15.0 \mathrm{MPa}$ over the 40 mm span with a minimum and maximum value of 10.7 and $17.1 \mathrm{MPa}$. Other batches of M1 showed considerable variation in strength within each batch. These batches were considered defective, probably due to improper homogenisation, and therefore not representative of the actual properties of composition M1. However, it should be noted that although most low-strength samples showed similarly low strength on both spans, some samples showed an extreme strength variation between the two 
Table 4.5: Variability of flexural properties between different batches of composition M1 all prepared by the same fabrication process and conditions ( \pm standard deviation)

\begin{tabular}{rrrr}
\hline & \multicolumn{1}{c}{$\begin{array}{c}\boldsymbol{\sigma}_{\mathbf{f}} \\
{[\mathbf{M P a}]}\end{array}$} & \multicolumn{1}{c}{\begin{tabular}{c}
\multicolumn{1}{c}{$\mathbf{E}_{\mathbf{f}}$} \\
{$[\mathbf{G P a}]$}
\end{tabular}} & $\begin{array}{c}\boldsymbol{\varepsilon}_{\mathbf{f}} \\
{[\mathbf{\%}]}\end{array}$ \\
\hline M1-1 & $4.7 \pm 2.5$ & $8.9 \pm 1.2$ & $0.05 \pm 0.02$ \\
$\mathbf{M 1 - 2}$ & $10.1 \pm 1.9$ & $10.0 \pm 0.3$ & $0.10 \pm 0.02$ \\
$\mathbf{M 1 - 3}$ & $10.6 \pm 1.3$ & $8.5 \pm 0.1$ & $0.12 \pm 0.02$ \\
$\mathbf{M 1 - 4}$ & $10.0 \pm 0.9$ & $8.2 \pm 0.4$ & $0.12 \pm 0.01$ \\
M1-5 & $9.4 \pm 3.7$ & $8.4 \pm 0.4$ & $0.11 \pm 0.05$ \\
M1-6 & $8.1 \pm 5.7$ & $8.8 \pm 0.2$ & $0.09 \pm 0.06$ \\
\hline
\end{tabular}

spans and also between the two bar pieces of the same bar sample on the $40 \mathrm{~mm}$ span. To clarify this aspect, the following three strength values of an exemplarily sample of sample series M1-5 are given: 3.4 MPa (100 mm), 2.7 MPa (40 mm) and 14.4 $\mathrm{MPa}(40 \mathrm{~mm})$. This indicates some major inhomogeneities even within a single bar specimen. Irrespective of the strength, the elastic modulus of all batches of composition M1 was relatively similar. Although the higher elastic modulus of batch M1-2 could not be reproduced a typical average elastic modulus of $8.3-8.8 \mathrm{GPa}$ was achieved for most batches of this composition. A good reproducibility of the elastic modulus was also observed for all other compositions with the variation of the average value between different batches generally within $0.5 \mathrm{GPa}$. The variability within each batch was also typically $<0.5 \mathrm{GPa}$ for all compositions.

Table 4.6: Comparison of the flexural properties of the strongest batch of compositions M1 - M5

\begin{tabular}{lrrc}
\hline & \multicolumn{1}{c}{$\begin{array}{c}\boldsymbol{\sigma}_{\mathbf{f}} \\
{[\mathbf{M P a}]}\end{array}$} & \multicolumn{1}{c}{$\begin{array}{c}\mathbf{E}_{\mathbf{f}} \\
{[\mathbf{G P a}]}\end{array}$} & $\begin{array}{c}\boldsymbol{\varepsilon}_{\mathbf{f}} \\
{[\mathbf{\%}]}\end{array}$ \\
\hline $\mathbf{M 1}$ & $10.6 \pm 1.3$ & $8.5 \pm 0.1$ & $0.12 \pm 0.02$ \\
$\mathbf{M} 2$ & $13.5 \pm 0.7$ & $9.2 \pm 0.2$ & $0.15 \pm 0.01$ \\
$\mathbf{M 3}$ & $9.6 \pm 1.9$ & $5.9 \pm 0.4$ & $0.16 \pm 0.04$ \\
M4 & $9.4 \pm 1.8$ & $6.3 \pm 0.1$ & $0.15 \pm 0.03$ \\
M5 & $16.4 \pm 1.0$ & $10.5 \pm 0.1$ & $0.16 \pm 0.01$ \\
\hline
\end{tabular}

The flexural properties of the five different geopolymer matrix compositions are compared in Table 4.6. Displayed are the batches that achieved the highest average strength on the $100 \mathrm{~mm}$ testing span. The reasons for this assumption were already briefly described in the previous section and will also be addressed in more detail in 
the discussion at the end of this chapter. The number of samples for flexural testing of compositions M2 - M5 was significantly smaller and only two batches of M2, M3 and M5 and one batch of M4 were tested. M5 showed the highest strength of all compositions with an average strength of $16.4 \mathrm{MPa}$ for the best batch. The highest overall strength measured for M5 and any other composition was $18.3 \mathrm{MPa}$ on a 40 mm span. The weaker batch of M5 was still significantly stronger than all other compositions with an average strength of $15.1 \mathrm{MPa}$. The average elastic modulus ranged between $10.5-11 \mathrm{GPa}$. The second highest average strength of $13.5 \mathrm{MPa}$ was recorded for composition M2. However, with an average strength of $9.2 \mathrm{MPa}$, the second batch was considerably weaker. Yet, the strength is too high to consider this batch as defective. Therefore, the strength of composition M2 is somewhat uncertain. The average strength values for the $40 \mathrm{~mm}$ span were similar to the ones stated before for the $100 \mathrm{~mm}$ span. The elastic modulus showed better reproducibility with average values between $9-9.5 \mathrm{GPa}$. Compositions M3 and M4 showed a roughly similar strength based on the comparison of the strongest batch of each composition. The average strength of the second batch of M3 was somewhat lower with a value of 8.3 MPa. Because only one batch of M4 was measured no further differentiation between the two mixtures can be made. The average elastic modulus of M3 appeared slightly lower compared to M4 with values ranging between 5.5 - 6 $\mathrm{GPa}$ and 6.3 $\mathrm{MPa}$, respectively.

To evaluate the influence of the drying time on the flexural properties, one batch of M1 was tested after 28 days for comparison. The average strength of this batch was somewhat lower achieving 9.3 MPa for the $100 \mathrm{~mm}$ span. For the $40 \mathrm{~mm}$ span an average strength of $10.5 \mathrm{MPa}$ was measured. However, these values are generally within the strength range of M1 as described above. The average elastic modulus of $8.9 \mathrm{GPa}$ is also similar.

\subsubsection{Effect of mixing time}

The fabrication process for the composite samples used a continuous mechanical mixing process in order to extend the effective use-time of the geopolymer binder and allowing the production of three composite samples from each batch of 
geopolymer binder. However, this meant that only the first composite sample used a geopolymer binder that was prepared according to the same parameters as the unreinforced specimens. All subsequent composites fabricated within the same batch used a matrix that was subjected to considerably longer mixing times. Therefore, several tests were carried out to investigate the influence of the mixing time on the compressive and flexural strength of the unreinforced geopolymer matrix. The standard homogenisation time was five minutes. The fabrication of each composite sample took roughly 20 minutes. Therefore, homogenisation times of 25 and 45 minutes were chosen to approximately match the mixing times of the geopolymer binder for the three composite samples of a fabrication series. The experiments were carried out on composition M1.

Despite the longer mixing time and supposedly better homogenisation, most of the produced sample batches were defective and achieved very low average strength values. The variability was similar to what has been described before for M1 samples prepared after standard homogenisation time. Only one batch of bar samples produced good results. An average flexural strength and modulus of $11.0 \pm 0.6 \mathrm{MPa}$ and $9.2 \pm 0.1 \mathrm{GPa}$ were measured on a $100 \mathrm{~mm}$ span for samples made after 25 minutes homogenisation time. Only one batch of compressive cylinders that indicate the influence of the mixing time produced valid results. Four samples could be cast from the left over binder after the fabrication of a series of composite specimens. The mixing time was slightly longer than intended and close to 60 minutes. The sample batch achieved an average compressive strength of $89.2 \mathrm{MPa}$ with three of the four samples showing very high strength ranging between 91 - $99 \mathrm{MPa}$. The strength of the fourth sample (74.4 MPa) was similar to the strength achieved by the two best batches after the standard homogenisation time of five minutes.

\subsubsection{Discussion}

The characterisation of the mechanical properties of the different geopolymer matrix compositions and the effect of various parameters on these properties was one of the main points of interest in this first part of the project. However, the lack of adequate reproducibility resulting in a large number of defective and underperforming 
specimens somewhat diminishes the significance of the obtained results despite the fact that some very good results could be achieved, too. It also makes a meaningful analysis of the results and their comparison to existing data difficult. Nevertheless, some aspects relating to the mechanical testing and the properties of geopolymers are discussed in more details below.

One factor that has to be considered is the fact that not the same number of batches/specimens was tested for all compositions. Due to its use as the main matrix composition for composite specimens, more samples of composition M1 were tested. Therefore, the results obtained for compositions M2 - M5 can only be considered as indicative. But despite the large number of specimens tested for composition M1, a statistical analysis of the results was not conducted due to the large inconsistency of the obtained results. The possibility that this inconsistency is in fact an extremely large statistical variation of the strength of the geopolymers is dismissed. Although it can be assumed that geopolymers, similarly to other ceramic materials, show a relatively wide statistical strength distribution around a maximum value, the variability between individual samples within the same batch was generally relatively small and in no relation to the variation between the different batches. Therefore, the strength variation between the different batches was clearly affected by some other factors than mere statistical variation. Consequently, a statistical evaluation of all results would have resulted in a false representation of the material properties. On the other hand, the number of well-performing specimens was too small for a meaningful statistical analysis and the clear identification of significant samples carried the risk of compromising any analysis in its own right. As a result, only the best performing sample batch for each testing method was chosen as the basis for comparison as it was assumed that these values present a more accurate representation of the true strength of the various geopolymer compositions. The weaker batches, on the other hand, were considered to be defective in one way or another and non-representative of the material properties. 
Table 4.7: Comparison between chemical composition and mechanical properties

\begin{tabular}{cccccrr}
\hline & \multicolumn{2}{c}{ Composition / molar ratios } & \multicolumn{1}{c}{$\boldsymbol{\sigma}_{\mathbf{c}}$} & \multicolumn{1}{c}{$\boldsymbol{\sigma}_{\mathbf{f}}$} & \multicolumn{1}{c}{$\mathbf{E}_{\mathbf{f}}$} \\
& $\mathbf{S i O}_{\mathbf{2}} / \mathbf{A l}_{\mathbf{2}} \mathbf{O}_{\mathbf{3}}$ & $\mathbf{N a}_{\mathbf{2}} \mathbf{O} / \mathbf{S i O}_{\mathbf{2}}$ & $\mathbf{H}_{\mathbf{2}} \mathbf{O} / \mathbf{N a} \mathbf{2} \mathbf{O}$ & \multicolumn{1}{c}{$[\mathbf{M P a}]$} & \multicolumn{1}{c}{$[\mathbf{M P a}]$} & \multicolumn{1}{c}{$[\mathbf{M P a}]$} \\
\hline $\mathrm{M} 1$ & 3.0 & 0.36 & 10.1 & $78.9 \pm 6.5$ & $10.6 \pm 1.3$ & $8.5 \pm 0.1$ \\
$\mathrm{M} 2$ & 3.3 & 0.37 & 10.2 & $74.9 \pm 7.4$ & $13.5 \pm 0.7$ & $9.2 \pm 0.2$ \\
M3 & 3.0 & 0.23 & 15.8 & $52.6 \pm 5.5$ & $9.6 \pm 1.9$ & $5.9 \pm 0.4$ \\
M4 & 3.0 & 0.36 & 11.7 & $68.2 \pm 5.5$ & $9.4 \pm 1.8$ & $6.3 \pm 0.1$ \\
M5 & 3.6 & 0.30 & 11.3 & $135.6 \pm 5.8$ & $16.4 \pm 1.0$ & $10.5 \pm 0.1$ \\
\hline
\end{tabular}

Table 4.7 summarises the compressive and flexural properties of the strongest sample series of each geopolymer mixture and compares the mechanical properties to the chemical composition. Although all composition achieved reasonably good strengths, the chemical composition had significant influence on the mechanical properties. The comparatively lower strength of composition M3 was to be expected due to the high $\mathrm{H}_{2} \mathrm{O} / \mathrm{Na}_{2} \mathrm{O}$ ratio, well above the ideal $\mathrm{H}_{2} \mathrm{O} / \mathrm{Na}_{2} \mathrm{O}$ ratio of around $10-$ 11 proposed in the literature $[23,90]$. The negative effect of the water content on the strength of geopolymers is well known. While a minimum amount of water is needed to facilitate the geopolymer reaction, too high water contents lead to an increasingly porous and weak geopolymer network. The effect of the increase of the water content on the strength of the cured geopolymer is also evident by direct comparison of compositions M1 and M4 since the only difference between both compositions was the water content. However, the low alkali content of composition M3 most likely contributed also to the relatively lower strength as well as the decreased hardness. Composition M3 showed in fact a deficiency of alkali ions (i.e. $\mathrm{Na} / \mathrm{Al}<1$ ). Thus, not all possible tetrahedral aluminium sites could be charge balanced by sodium ions resulting in an incomplete reaction and weakened geopolymer network. Except for M3, all other compositions were largely within the proposed optimal compositional range for strong geopolymers: $\mathrm{SiO}_{2} / \mathrm{Al}_{2} \mathrm{O}_{3} \sim 3-4$, $\mathrm{M}_{2} \mathrm{O} / \mathrm{SiO}_{2} \sim 0.25-0.4$ and $\mathrm{H}_{2} \mathrm{O} / \mathrm{M}_{2} \mathrm{O} \sim 10-11 ; \mathrm{M}: \mathrm{Na}^{+}$or $\mathrm{K}^{+}[23,90]$, as described in chapter 2.2.3. Although all of these compositions could produce strong geopolymers, the superior performance of M5 clearly shows that the best mechanical properties can be achieved for compositions with $\mathrm{SiO}_{2} / \mathrm{Al}_{2} \mathrm{O}_{3}$ ratios closer to four and a balanced a balanced $\mathrm{Na} / \mathrm{Al}$ ratio $(\mathrm{Na} / \mathrm{Al}=1)$. The positive effect of the higher $\mathrm{SiO}_{2} / \mathrm{Al}_{2} \mathrm{O}_{3}$ ratio is consistent with previous observations in the literature [56]. The 
positive effect of the slightly increased $\mathrm{SiO}_{2} / \mathrm{Al}_{2} \mathrm{O}_{3}$ ratio is also indicated by the higher flexural strength of composition M2 compared to M1. However, the same could not be observed for the compressive strength. Although the possibility of a lower compressive strength for composition M2 cannot be ruled out completely, it is most likely that a higher compressive strength was simply not achieved as a result of the reproducibility issues and the small number of batches that were tested for this composition. Thus, it is believed that composition M2 should at least in theory be stronger than M1.

As mentioned before, the results that were obtained in the splitting-tensile test were largely invalid due to the presence of cracks in a large number of specimens. Nevertheless, some conclusions could be drawn from the results of the un-cracked samples. The initial tests clearly showed the effect that the general processing and fabrication methods have on the mechanical properties. By improving these processes the average strength of composition M1 could be gradually improved by about $50 \%$ from approximately $3 \mathrm{MPa}$ for initial hand mixed batches to $\sim 4.4 \mathrm{MPa}$ using the mechanical mixing process. However, because these early samples were not analysed for the presence of any microcracks the question arose if these specimens were indeed crack-free as originally presumed or contained microcracks like most of the subsequent sample batches. Since the only crack free batch of pellets that was subsequently produced achieved an average splitting-tensile strength of 4.2 MPa it can be assumed that these early batches were also crack free. Therefore, an average tensile strength of approximately $4.4 \mathrm{MPa}$ can be assumed for composition M1.

The influence of the mixing and drying time on the flexural and compressive strength was briefly investigated on composition M1. The investigation of the effect of the mixing time was overshadowed by a large output of obviously defective specimens achieving very low strength. This was somewhat surprising since the longer mixing times were expected to increase the homogeneity of the geopolymer binder and, thus, improve reproducibility and mechanical performance. This may indicate that the problem of reproducibility is somewhat more complex and may not be reduced 
simply to the lack of insufficient homogenisation. The one batch of bar specimens that produced reasonable results achieved an average flexural strength of $11 \mathrm{MPa}$ after a homogenisation time of 25 minutes. This is only a minor increase of the flexural strength compared to the results that were achieved for specimens after the standard five minute homogenisation time. The only samples that achieved valid results for compressive testing were prepared from left over binder after the fabrication of composite specimens. The binder was subjected to a homogenisation time of nearly 60 minutes. Three out of four specimens achieved a compressive strength of $91-99 \mathrm{MPa}$, showing a significant increase of the compressive strength compared to the standard homogenisation time. In terms of the effect of drying time, the compressive strength showed an expected increase over time achieving an average strength of $\sim 95 \mathrm{MPa}$ after 28 days. The average flexural strength of the sample batch tested after 28 days was slightly lower than the best batch tested after seven days whereas the elastic modulus was slightly higher (8.9 vs. $8.5 \mathrm{GPa}$ ). However, the elastic modulus may be the better indicator here and the actual strength value may be underrepresented. Since an actual decrease in flexural strength over time seems rather unlikely, it was assumed that the flexural strength is not majorly affected by extended drying times. This observation is supported by the splittingtensile test results. The two batches that achieved an average tensile strength of 4.4 MPa were tested after a drying time of 16 days. However, an average strength of 4.2 $\mathrm{MPa}$ was achieved after only two days drying time. Although the data is arguably slim and further investigations of the influence of these parameters are certainly required, the results indicate the conclusion that the two parameters, mixing and drying time, appear to have different effects on the compressive and the tensile/flexural strengths. While extended mixing and drying times can lead to a significant increase in compressive strength, the tensile/flexural strength seems to be hardly affected in both cases. At least with regard to the drying time, these results contradict previous observations made by Wang et al. for a similar sodium-activated metakaolin system [93].

The failure behaviour of all bar specimens was linear elastic with typical brittle failure originated on the tensile side of the bar. Three types of fracture surfaces could 
be observed: i) plane fracture through the thickness of the bar, ii) plane fracture with a curvature of the fracture surface towards the compression side and iii) random and uneven fracture surfaces. However, no conclusive relation between the appearance of the fracture surface and the measured strength could be established. Fracture surfaces of type i) and ii) were the most common and could be observed for strong as well as defective specimens. Fracture surfaces of type iii) were only found in weak and defective specimens. In regards to the point of fracture, the majority of the defective specimens did not fail at the central loading point but at random points across the support span. While most of the strong specimens failed, as expected, in close proximity to the point of central loading, this clearly indicates the presence of some sort of structural defects in a large number of specimens. A more detailed analysis of the microstructure on the fracture surfaces is presented in the chapter 4.3.

As mentioned before, the comparison of results between different studies is generally difficult. However, some form of comparison is necessary to put the obtained results into perspective. Taking only the best-performing sample batches for each test method and matrix composition, the results of the various mechanical properties measurements are generally very good. Since the compressive strength is the most widely reported parameter of the mechanical properties throughout the geopolymer literature, a general comparison has to be based on this parameter. In that regard, composition M5 with an average compressive strength of $>135 \mathrm{MPa}$ showed an exceptional performance. Although geopolymers with compressive strengths $>100$ MPa have been reported before [92], such high strengths are rarely reported and are typically only achieved with fly ash- and/or slag-based geopolymers.

In Table 2.2 the mechanical properties and chemical composition of four selected metakaolin-based geopolymer matrices that were employed in previous studies on fibre reinforced geopolymer composites were presented. These materials are compared to the present compositions M1 and M5 in Table 4.8. The different matrices shown in Table 4.8 are compared and critically discussed in some more detail in the following section. Except for the type of alkali cation used in all four of these studies, the chemical composition of matrix M1 was relatively similar to the 
one by $\mathrm{He}$ et al. [13] whereas the composition of M5 was relatively similar to the ones used by Lin et al. [20] and Kriven et al. [23]. The somewhat lower flexural strength and modulus of composition M1 compared to He et al. [13] may be attributed to the slightly higher alkali content of M1, the material system that was used as well as different processing and testing parameters. The flexural strength of composition M5 matches very well with the strength reported by Lin et al. [20]. Thus, both studies seem to support the results achieved in the present study and back up the previously made assumption that the strongest batches for each composition and test method represent a better indication of the true strength of these materials.

Table 4.8: Comparison of mechanical properties of compositions M1 and M5 with other metakaolin geopolymer matrices reported in the literature

\begin{tabular}{lcccccc}
\hline & {$[9]$} & {$[23-25]$} & {$[20]$} & {$[13]$} & $\mathrm{M} 1$ & $\mathrm{M} 5$ \\
\hline Composition & & & & & & \\
$\quad \mathrm{Alkali} \mathrm{Ion}\left(\mathrm{M}^{+}\right)$ & $\mathrm{K}^{+}$ & $\mathrm{K}^{+}$ & $\mathrm{K}^{+}$ & $\mathrm{K}^{+}$ & $\mathrm{Na}^{+}$ & $\mathrm{Na}^{+}$ \\
$\mathrm{SiO}_{2} / \mathrm{Al}_{2} \mathrm{O}_{3}$ & $\mathrm{n} / \mathrm{a}$ & 4 & 4 & 3 & 3.0 & 3.6 \\
$\mathrm{M}_{2} \mathrm{O} / \mathrm{SiO}_{2}$ & $\mathrm{n} / \mathrm{a}$ & 0.3 & 0.3 & 0.33 & 0.36 & 0.30 \\
$\mathrm{H}_{2} \mathrm{O} / \mathrm{M}_{2} \mathrm{O}$ & $\mathrm{n} / \mathrm{a}$ & 11 & 11 & 11 & 10.1 & 11.3 \\
& & & & & & \\
Tension & 3.7 & $\mathrm{n} / \mathrm{a}$ & $\mathrm{n} / \mathrm{a}$ & $\mathrm{n} / \mathrm{a}$ & $\sim 4.4$ & $\mathrm{n} / \mathrm{a}$ \\
$\quad$ Strength $\sigma_{\mathrm{t}}[\mathrm{MPa}]$ & & & & & & \\
$\quad \begin{array}{l}\text { Compression } \\
\text { Strength } \sigma_{\mathrm{c}}[\mathrm{MPa}]\end{array}$ & 39 & 45 & $\mathrm{n} / \mathrm{a}$ & $\mathrm{n} / \mathrm{a}$ & $\sim 79$ & $\sim 136$ \\
& & & & & & \\
Flexure & & & & & & \\
$\quad$ Strength $\sigma_{\mathrm{f}}[\mathrm{MPa}]$ & 8.1 & 1.7 & 16.8 & 12.3 & 10.6 & 16.4 \\
$\quad$ Modulus $\mathrm{E}_{\mathrm{f}}[\mathrm{GPa}]$ & 9.4 & $\mathrm{n} / \mathrm{a}$ & $\mathrm{n} / \mathrm{a}$ & 10.3 & 8.5 & 10.5 \\
\hline
\end{tabular}

However, the reported strength values in the two studies by Lin et al. [20] and He et al. [13] were obtained from a three-point bending test with sample dimensions of $3 \mathrm{x}$ $4 \times 36 \mathrm{~mm}$ and a loading speed of $0.5 \mathrm{~mm} / \mathrm{min}$. These parameters, i.e. sample dimensions, and loading rate, differ significantly from the ones used in the present study. Therefore, the feasibility of these results and the above comparison has to be evaluated. For that purpose, the results by Wang et al. shall be considered [93]. This author investigated the effects of the concentration of the alkali activator solution and drying time on the compressive and flexural strength in a sodium-activated metakaolin system. For a $12 \mathrm{M} \mathrm{NaOH}$ activator solution, similar to the one used in 
the present study, this author reported a flexural strength of $>50 \mathrm{MPa}$ after seven days and a corresponding compressive strength of $\sim 65 \mathrm{MPa}$. While the compressive strength is similar to the strength achieved for composition M4 in this study and seems indeed feasible, the flexural strength is more than five times the value measure for composition M4 and appears largely unrealistic. When comparing the testing parameters, the sample dimensions $(13 \times 26 \mathrm{~mm})$ for the compressive strength test were found to be roughly similar to the ones used in the present study. The specimens for the flexural testing, however, were significantly smaller, measuring only $6 \times 7 \times 30 \mathrm{~mm}$. The extremely short length combined with a comparatively big thickness and width is the most probable cause for this misrepresentative high strength value and emphasizes the importance of choosing the right parameters to obtain meaningful results. Although the length of the specimens used by Lin et al. [20] and He et al. [13] is also relatively small, the width and thickness are smaller, too. While the use of such small specimens is relatively common for the flexural strength testing of engineering ceramics, their applicability for geopolymers seems challenging due to the fragility and relatively low mechanical strength of geopolymers compared to engineering ceramics. Nonetheless, unlike the flexural strength reported by Wang et al. [93], the measurements conducted by Lin et al. [20] and He et al. [13] seem to obtain reasonable results. Therefore, the initial comparison between the results obtained by these authors and the present results for compositions M1 and M5 seems to be sensible. On the other hand, the comparison of the mechanical properties of composition M5 and Lin et al. [20] with Kriven et al. [23] in Table 4.8 exhibits major discrepancies. The nature and reasons for this apparent difference shall be explored in more detail below.

As mentioned before, the chemical composition of the geopolymers used by Kriven et al. [23] and Lin et al. [20] are identical and are relatively close to composition M5. It is accepted, that all three of these composition are close to what is widely considered the optimum compositional range for strong geopolymers. As discussed above, the flexural properties of Lin et al. [20] are very similar to composition M5. Although there is no general relationship between the compressive and flexural strength of geopolymers, a roughly similar compressive strength would also be 
expected. Given the similarity in the chemical compositions, similar properties would also be expected for the geopolymer used by Kriven et al. [23]. However, the comparison of the strength values reveals major discrepancies. Although there are many factors that can affect the mechanical properties such as the material system, processing and testing parameters, etc., it seems unlikely that these factors would account for such a major discrepancy between the three studies. Therefore, other possible explanations for the comparatively low strength values of Kriven et al. have to be considered. Based on the observations made in the present study, a number of possible reasons shall be explored that may help to explain this discrepancy between the strength values.

The first possibility is that the low mechanical strength is a result of insufficient processing of the geopolymer binder. This argument is supported by the observation within the same publication that by improving the general processing and the application of a vacuum method to remove entrapped air, the compressive strength could be improve up to a maximum value of $83 \mathrm{MPa}$. Although this value would still appear relatively low compared to the strength achieved for composition M5, it clearly highlights the effect of the processing methods on the mechanical properties. However, the vacuum method was chosen not to be used for the main part of the study by Kriven et al. [23]. Thus, the presence of large pores and/or inhomogeneities due to insufficient homogenisation of the binder may act as defect sites in the sample and cause a significant weakening of the material.

Another possible explanation is the overall specimen size. In the case of Kriven et al. [23] rather large bar specimens were used for flexural testing with approximate dimensions of $25 \times 25 \times 150 \mathrm{~mm}$. As discussed before, problems with the development of drying cracks were observed for pellet specimens in the present study. It was concluded that the cracking may be related to the sample size since similar problems were not observed in the smaller bar and cylinder specimens. Therefore, a cross-sectional area of roughly $10 \times 10 \mathrm{~mm}$ or smaller was suggested to avoid sample cracking. Furthermore, it was observed that a large number of visible cracks seemed to disappear within $24-48$ hours after their first occurrence. The 
presence of a larger number of previously undetected microcracks was also found in most of these specimens. Hence, it may be possible that similar issues are responsible for the low flexural strength. However, this would not explain the relatively low compressive strength because the cylinder specimens $(6 \times 25 \mathrm{~mm})$ used by Kriven et al. [23] were even smaller than the ones used in the present study. That is, unless the casting process of such narrow specimens would have caused problems with the sample quality. From the experience of moulding $10 \mathrm{~mm}$ wide cylinders in the present work, casting such small specimens would be considered very difficult and would require a very low viscosity of the binder.

The theory of localised composition gradients or other types of inhomogeneities (other than large pores) as critical defect sites was suggested before to explain the large variation between the different sample batches in the present study. Regardless of what causes these defects, the same theory could be applied to explain the results by Kriven et al. [23]. In fact, the mechanical properties reported by this author are very similar to the ones measured for some of the weak sample batches of composition M1 which typically achieved compressive and flexural strengths of $30-$ $40 \mathrm{MPa}$ and $1-3 \mathrm{MPa}$, respectively. Without more information the true reasons for this discrepancy can only be speculated. However, the above considerations entertain the suspicion that the low strength values reported by Kriven et al. [23] could be based on the measurement of in some way defective specimens. The significant differences between the strengths of different sample batches observed in this study support this theory. Therefore, it is suggested that the strength values reported by Kriven et al. [23] may be largely underrepresented and do not reflect the true properties of the material accurately. In this context, the study by Kriven et al. [23] is merely an example and the same considerations may also apply to a large number of other studies. This would also explain why the problem of reproducibility, which was the main concern of this part of the present study, has not been discussed in the existing literature. This behaviour was only observed due to the large number of batches that were measured for the same composition (M1). If only one batch had been measured for each composition, irrespective of the number of specimens per batch, then the probability that any of these batches would have been defective and 
weak is relatively large. Thus, the measured strength could have easily been mistaken as the apparent material property, resulting in a misrepresentation of the true properties of the particular composition as well as a possibly false conclusion on the influence of various parameters such as the chemical composition on the mechanical properties. Based on these hypotheses, the mechanical properties of metakaolin geopolymers are believed to be widely misrepresented and the current understanding of these materials may have to be re-evaluated.

\subsection{Microstructure}

\subsubsection{SEM and EDS}

The microstructure of all five geopolymer compositions was analysed on fracture surfaces of bar specimens by means of electron microscopy and EDS analysis. Figure 4.5 shows typical high magnification images of the microstructure in well-reacted areas for each composition. The microstructures of all five compositions in these areas were relatively similar and resemble typical geopolymer microstructures. However, at closer inspection some differences could be identified. Small roundedged geopolymer gel particles with a typical size of 100 to $500 \mathrm{~nm}$ can be observed in all micrographs of Figure 4.5. These particles were connected to various degrees to form a more or less porous structure. The SEM analysis showed that the structure of composition M3 was mainly made up of small discrete geopolymer gel particles and appeared to be the least dense. This was consistent with the comparatively higher roughness of the fracture surface which could also be observed at lower magnification. The microstructures of composition M1 and M4 were very similar and showed a slightly denser structure than M3. Larger areas of relatively dense geopolymer gel phase could be observed embedded in a structure of small discrete geopolymer particles as described before for M3. Composition M5 showed the densest microstructure and the highest smoothness of the fracture surface. The geopolymer gel phase appeared densely packed over large parts of the sample and only small areas of discrete particle structures were found. Composition M2 showed 

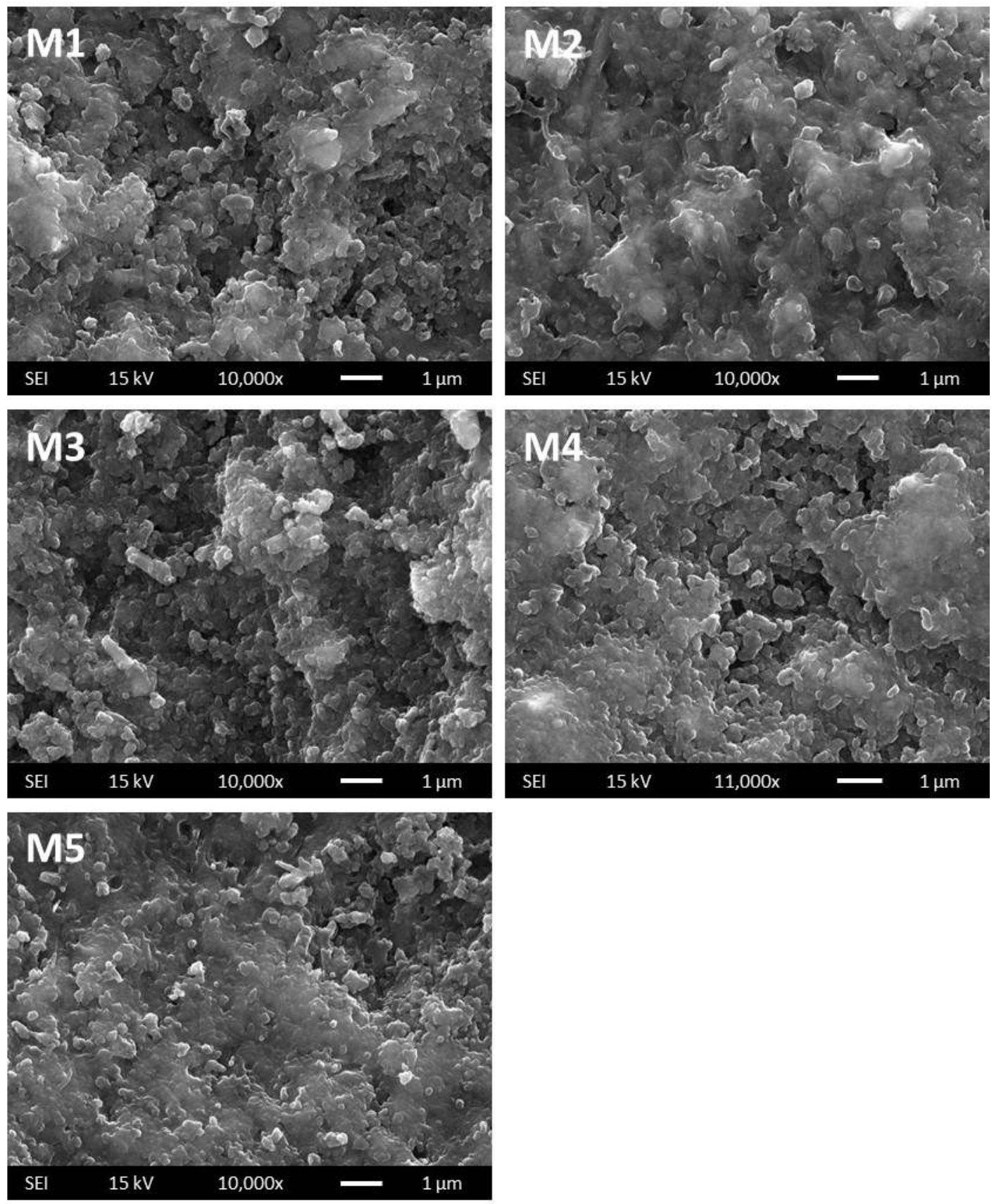

Figure 4.5: High magnification images of fracture surfaces, displaying typical microstructures of well reacted areas of geopolymer compositions M1 - M5

the biggest dissimilarity in the appearance of the microstructure of the five compositions. The structure of M2 generally appeared less defined and glassier with softer edges. As mentioned before, the microstructures shown in Figure 4.5 are only representative for well-reacted areas of each sample. Whereas this applied to large parts of the samples of M1, M3, M4 and M5, the microstructure described above for composition M2 only represented some smaller regions of the total sample area. The 
particular characteristics of the microstructure of composition M2 are described in more detail further on in this chapter.

Despite a general variation in the overall roughness of the fracture surfaces for the different compositions, an irregular pattern of small lowered and elevated areas could be observed for all samples including M2. This is shown on a low magnification image of the fracture surface of sample M5 in Figure 4.6(a). The examination of the same picture in backscattered mode revealed that most of the lowered and elevated areas appeared in a lighter colour compared to the rest of the sample, indicating the presence of some inhomogeneities with a somewhat different chemical composition (Figure 4.6(b)). The corresponding EDS map in Figure 4.6(d) shows a relatively homogeneous distribution of sodium, silicon and aluminium over the sample surface with the exception of the lighter areas. These areas displayed a comparatively higher concentration of aluminium and lower sodium content as indicated by the colour difference in the EDS map. The Si content in these areas was similar to the surrounding phase. This compositional variation was consistent with a slight difference in the microstructural appearance of the sodium poor areas compared to the bulk of the sample (Figure 4.6(c)). The EDS spot analyses indicated the difference in the chemical composition of the two phases with regard to the $\mathrm{SiO}_{2} / \mathrm{Al}_{2} \mathrm{O}_{3}$ ratio (Figure 4.6(c)). The microstructure of the sodium poor areas generally appeared to be characterised by a higher degree of small discrete geopolymer gel particles similar to the microstructure that was observed for composition M3 (see Figure 4.5). The degree to which the microstructure in these areas differed from the structure of the main geopolymer phase depended on the geopolymer composition. The differences were more subtle for composition M3 than for composition M5. 

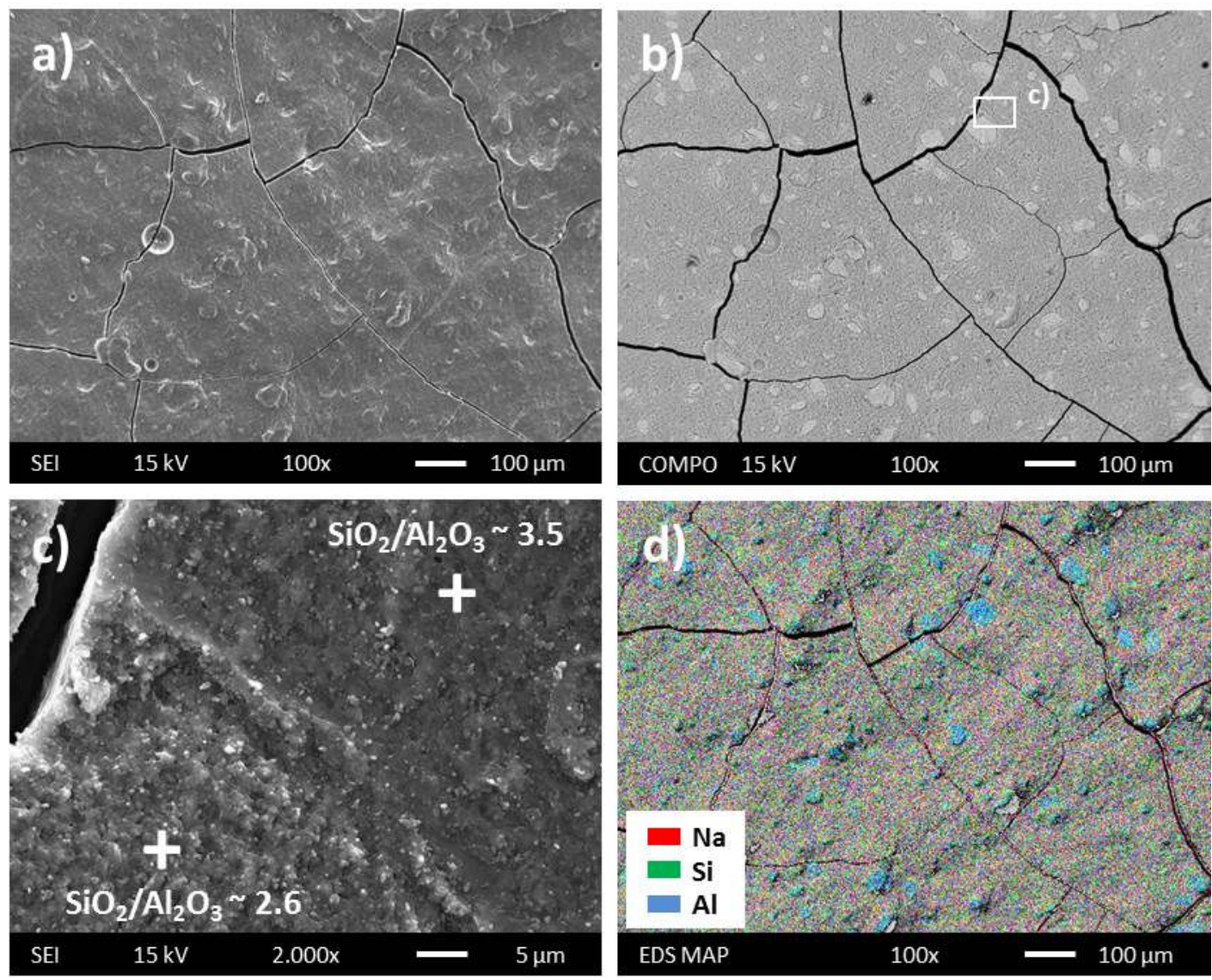

Figure 4.6: SEM analysis of fracture surface of M5 bar specimen: a) low magnification image showing typical irregular surface pattern of elevated and lowered areas, largely consistent with the lighter coloured areas in backscatter image (b); c) higher magnification image showing microstructural difference between lighter area and main phase. EDS spot analyses indicate the different composition with regard to the $\mathrm{SiO}_{2} / \mathrm{Al}_{2} \mathrm{O}_{3}$ ratio of the two phases; d) EDS elemental map of image b). The blue coloured areas indicate a comparatively lower sodium and higher aluminium concentration compared to the relatively homogeneous distribution of $\mathrm{Na}, \mathrm{Si}$ and $\mathrm{Al}$ in the surrounding main phase

The sample of composition M2 showed a largely inhomogeneous microstructure. As stated before, only small areas of the whole sample surface area showed a microstructure similar to that of the other compositions as shown in Figure 4.5-M2. The arrows in Figure 4.7(c) and (e) indicate such areas. The microstructure of the M2 sample was dominated by the two phases displayed in Figure 4.7(a) and (b) and Figure 4.7(c) and (d) at different levels of magnification. Both phases appeared to be amorphous but had a somewhat different morphological appearance. One phase showed a block-like structure (Figure 4.7(a) and (b)) whereas the other phase showed a more sheet-like appearance (Figure 4.7(c) and (d)). EDS spot-analysis suggested that both phases are chemically similar with an elevated sodium and considerably 
higher silicon content compared to the phase shown in Figure 4.5-M2. The aluminium content was found to be slightly lower. However, the chemical composition of these phases shall not be quantified here, due to some variability of the absolute element concentration in the EDS spot-analysis in different areas of the sample. A third phase that was repetitively found over large parts of the sample surface is shown in Figure 4.7(e). This seemingly crystalline phase was characterised
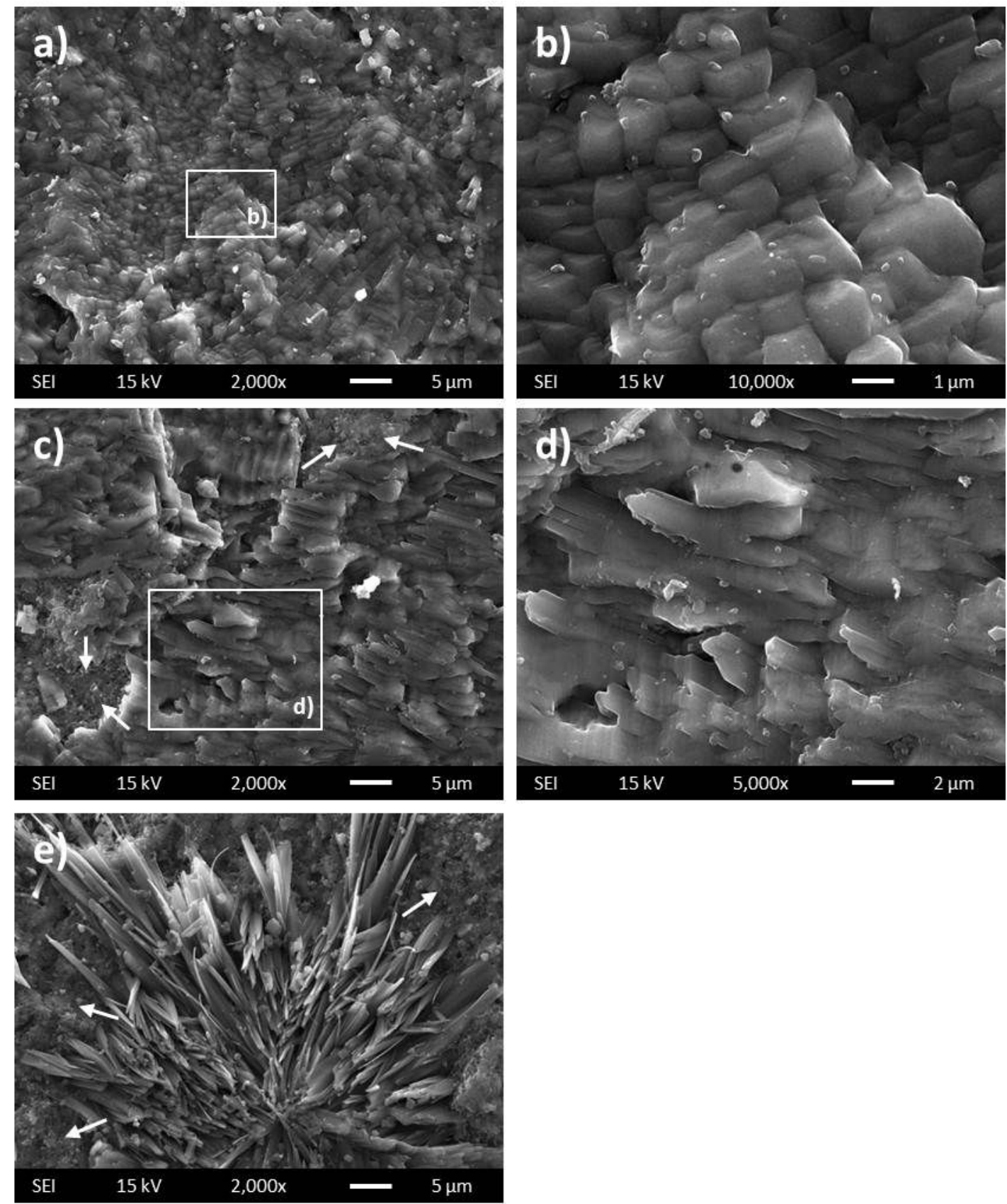

Figure 4.7: Phase inhomogeneities on fracture surface of composition M2. Arrows indicate microstructural areas as shown in Figure 4.5-M2 

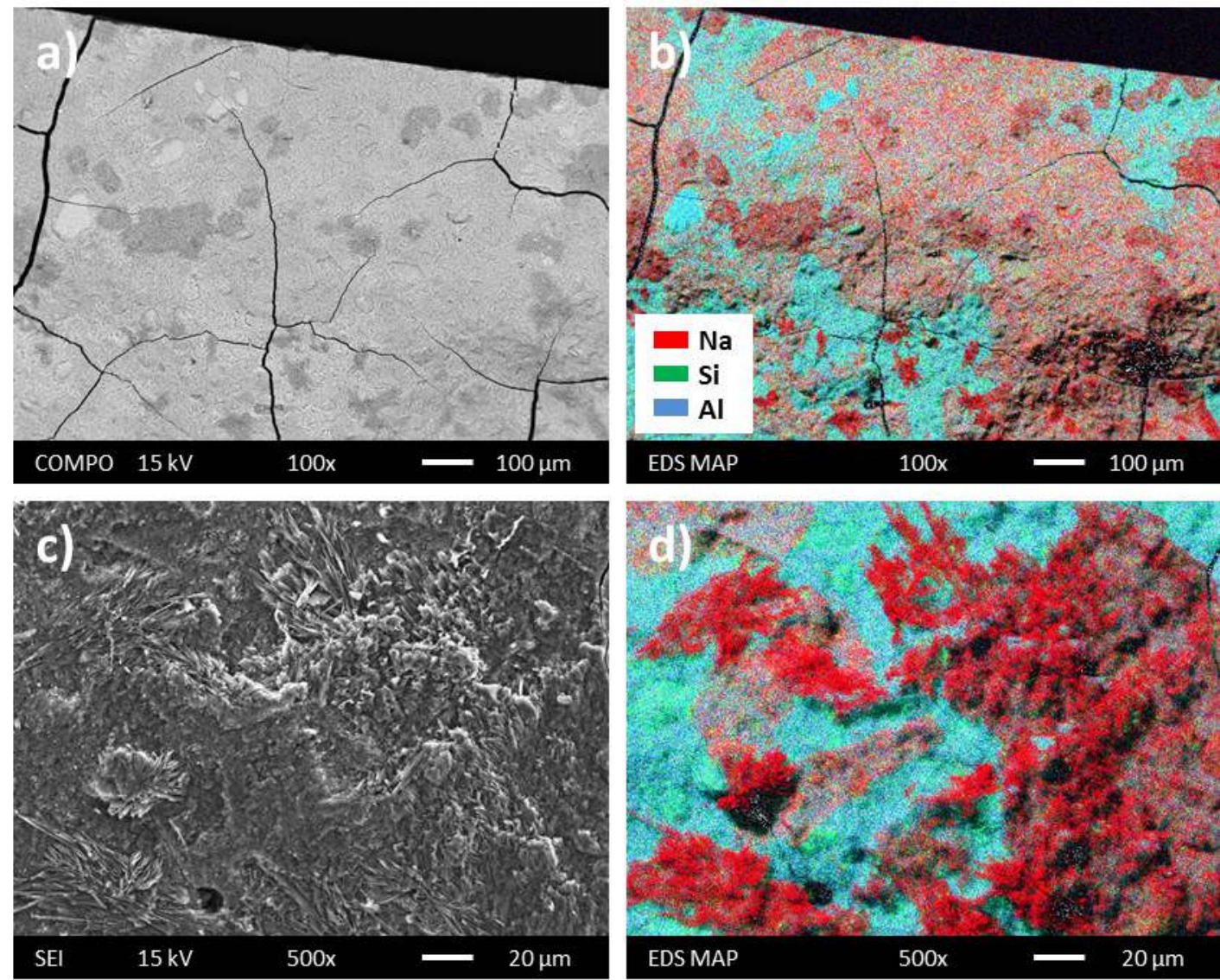

Figure 4.8: EDS mapping of various areas of fracture surface of M2 bar specimen: (a, b) low magnification image and EDS map showing a very inhomogeneous sodium distribution. The needle structures (c) are identified as very sodium rich phases by the EDS map (d)

by a mainly "flower"-like appearance consisting of fine needle and small platelet structures. EDS spot-analysis indicated a sodium-rich phase with smaller amounts of silicon and aluminium. The inhomogeneous microstructure and distribution of the three main elements $\mathrm{Na}, \mathrm{Si}$ and $\mathrm{Al}$ over larger areas of the M2 sample surface is shown in Figure 4.8 on the example of two low magnification micrographs and their respective EDS maps. The backscattered image in Figure 4.8(a) clearly reveals three different phases according to the colour brightness, i.e. a main phase with some lighter and some darker areas. The lighter areas were already described in Figure 4.6 and showed a low sodium concentration. The darker areas were largely consistent with the appearance of the flower structures described before (Figure 4.7(e)). The sodium richness of this phase is clearly indicated by the intensity of the red colour in both EDS maps shown in Figure 4.8. The EDS map in Figure 4.8(b) also reveals that the sodium distribution within the main phase is largely inhomogeneous. This is most 
evident in the top left and right corners and the bottom centre. Images (c) and (d) reveal that the more blue coloured part of the main phase can be attributed to the geopolymer phase as shown in Figure 4.5-M2 and the light red coloured phase is consistent with the two phases shown in Figure 4.7(a)-(d).

\subsubsection{XRD and NMR}

The x-ray diffraction patterns of powdered samples of geopolymer compositions M1 to $\mathrm{M} 5$ as well as the metahalloysite starting material after dehydroxylation at $600^{\circ} \mathrm{C}$ are presented in Figure 4.9. The diffraction pattern of the metahalloysite showed a typical broad hump at roughly $15-35^{\circ} 2 \theta$, indicating a largely x-ray amorphous structure, and several distinct peaks. These peaks could be attributed to quartz and cristobalite impurities in the metahalloysite. The diffractograms of the geopolymer

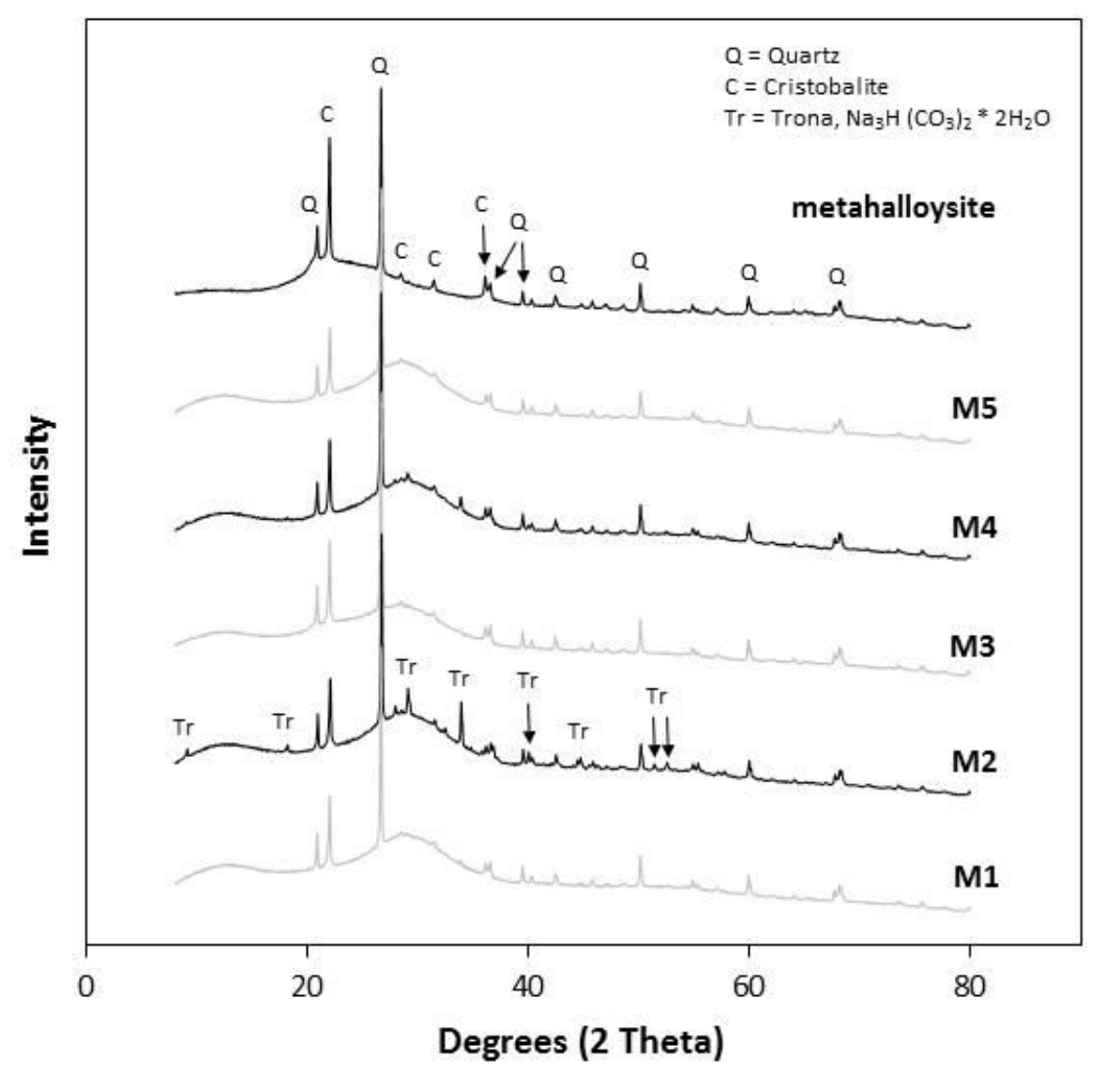

Quartz (PDF 01-089-8935) Cristobalite (PDF 04-007-2134) Trona (PDF 00-029-1447)

Figure 4.9: X-ray diffraction patterns for geopolymer compositions M1 - M5 and metahalloysite 
samples were all relatively similar and were also characterised by a broad amorphous hump between approximately $20-35^{\circ} 2 \theta$, typical for well-reacted geopolymers. However, the amorphous hump of sample M3 appeared somewhat flatter and wider compared to the other geopolymer compositions, possibly indicating a lower degree of reactivity. The crystalline impurities of the metahalloysite remained largely unreacted and their corresponding peaks were a common feature in all five geopolymer samples. Additional peaks were detected in samples of compositions M2 and M4. These peaks could be identified to match the diffraction pattern of trona, a hydrated sodium hydrogen carbonate.
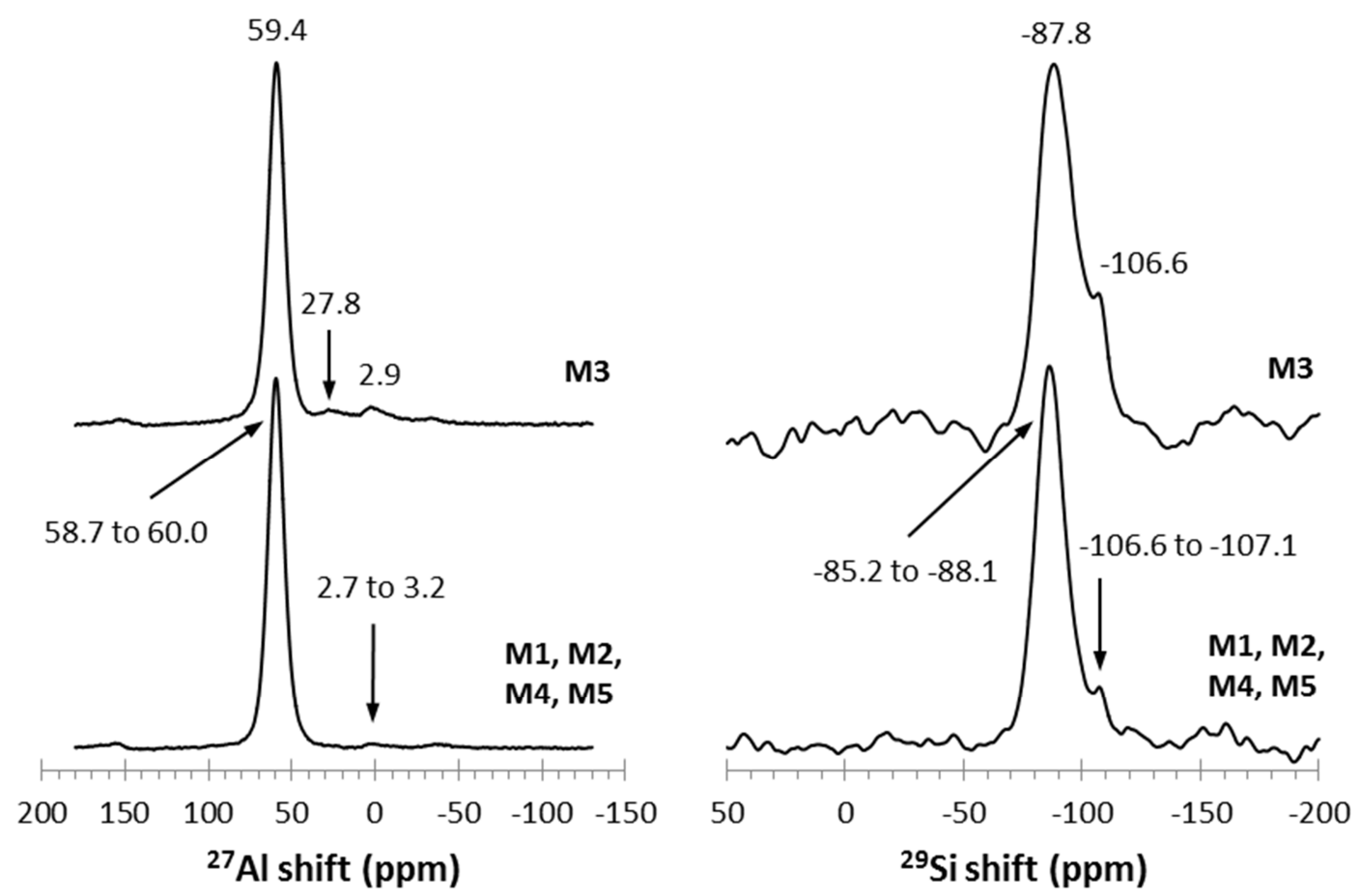

Figure 4.10: ${ }^{27} \mathrm{Al}$ and ${ }^{29} \mathrm{Si}$ MAS-NMR spectra of geopolymer compositions M1 - M5

Figure 4.10 presents typical ${ }^{27} \mathrm{Al}$ and ${ }^{29} \mathrm{Si}$ MAS NMR spectra of samples of compositions M1 - M5. The respective spectra of the different samples were very similar with the exception of the sample of composition M3. All ${ }^{27}$ Al MAS NMR spectra showed a major peak at around $59 \mathrm{ppm}$ which corresponds to tetrahedral aluminium in the geopolymer gel. The very small peak observed at $\sim 3 \mathrm{ppm}$ can be attributed to octahedrally coordinated $\mathrm{Al}$ which indicates the presence of trace 
amounts of unreacted metahalloysite. The intensity of the octahedral Al peak was slightly higher for M3 compared to the other compositions. The sample of composition M3 also showed a minor peak at $\sim 28 \mathrm{ppm}$ indicating a small amount of aluminium in $\mathrm{V}$-fold coordination which can also be attributed to residual metahalloysite. The ${ }^{29}$ Si MAS NMR spectra all featured a major peak at around -88 ppm and a clearly distinguishable smaller peak at the shoulder of the main peak at about $-107 \mathrm{ppm}$. Compared to the other compositions, the sample of M3 showed a markedly higher intensity of the secondary peak and a generally wider main peak.

\subsubsection{Discussion}

The SEM analysis of the microstructure on the fracture surfaces of bar specimens of compositions M1 and M3 - M5, as shown in Figure 4.5, suggested the formation largely well-reacted geopolymers with small differences in the microstructure between the different compositions. However, some small phase inhomogeneities that manifested as lighter areas under backscattered imaging conditions, as shown in Figure 4.6, were common to all samples. These areas generally showed somewhat higher aluminium and lower sodium contents compared to the main phase according to EDS analysis. Since the metahalloysite was the only aluminium source in this system, these areas were believed to be unreacted or partially reacted agglomerates of metahalloysite particles. At a closer look, the two phases also showed a slightly different microstructure indicating a different degree of reactivity (see Figure 4.6(c)). However, the microstructure of the sodium poor areas did not resemble any similarity with the microstructure of the metahalloysite agglomerates that were found after dehydroxylation and sieving, as was shown in Figure 3.2. Instead, the microstructure generally appeared to be characterised by a high degree of small discrete geopolymer gel particles similar to the microstructure that was observed for composition M3 (see Figure 4.5 and Figure 4.6(c)). The $\mathrm{SiO} 2 / \mathrm{Al}_{2} \mathrm{O}_{3}$ ratio of $\sim 2.6$ within these areas, determined by EDS spot analysis, was also significantly higher than the $\mathrm{SiO}_{2} / \mathrm{Al}_{2} \mathrm{O}_{3}$ ratio of $\sim 1.4$ of the unreacted metahalloysite. Therefore, it is concluded that the inhomogeneities were in fact partially reacted rather than unreacted agglomerates of metahalloysite with a somewhat lower $\mathrm{SiO}_{2} / \mathrm{Al}_{2} \mathrm{O}_{3}$ ratio and sodium content than the main geopolymer phase of each composition. 
The EDS spot analysis of the main geopolymer gel phase of composition M5 determined a $\mathrm{SiO}_{2} / \mathrm{Al}_{2} \mathrm{O}_{3}$ ratio of $\sim 3.5$ (see Figure 4.6). This value is very similar to the calculated $\mathrm{SiO}_{2} / \mathrm{Al}_{2} \mathrm{O}_{3}$ ratio of 3.6 for this composition. However, a slightly higher $\mathrm{SiO}_{2} / \mathrm{Al}_{2} \mathrm{O}_{3}$ ratio than the calculated value was expected as a result of the incomplete reaction of all metahalloysite particles. Due to the fact that some areas were only partially reacted, resulting in a geopolymer-like phase with a comparatively higher alumina content as explained before, not all of the aluminium from the metahalloysite was available for the formation of the main geopolymer phase. Conversely, the smaller amount of available aluminium in the main geopolymer phase should have therefore resulted in a comparatively higher $\mathrm{SiO}_{2} / \mathrm{Al}_{2} \mathrm{O}_{3}$ ratio. Possible explanations why this effect was not observed may be due to the possibility that the calculated compositional ratios may have been slightly higher than the actual ratios or some variability between the results of the EDS spot analysis in different places. In any case, the amount of aluminium that was not available for reaction was relatively small. Therefore, the difference between the actual and the calculated composition of the main geopolymer phase is considered to be relatively small.

Composition M5 showed the most homogeneous and densely packed microstructure with a seemingly high degree of interconnectivity in the geopolymer gel. This is in agreement with the high mechanical properties measured for this composition. On the other hand, the microstructure of composition M3 was characterised by interconnected but relatively discrete round-edged geopolymer gel particles. This microstructural appearance is believed to be the result of a somewhat lower degree of reaction due to the lack of sufficient amounts sodium ions to charge balance all existing aluminium sites. The seemingly lower packing density of the geopolymer gel phase is consistent with the lowest elastic modulus and strength for this composition. Compositions M1 and M4 showed very similar microstructures somewhere in between the microstructural appearance of compositions M3 and M5. The observation that larger areas of relatively dense geopolymer gel phase, similar to the microstructure of M5, could be found embedded in a network of smaller, more discrete geopolymer particles as described for M3 may support the hypothesis that 
the geopolymerisation behaviour is not consistent throughout the whole of the sample. This may lead to the formation of slightly different geopolymer phases within the sample depending on the locally available concentration of reactive silicon and alumina sites, alkali cations and water which control the geopolymerisation reaction in their immediate vicinity. On the other hand, a preferential phase formation may also be possible with different phases forming at different times throughout the geopolymerisation process. In any case, more detailed investigations of the general geopolymerisation behaviour beyond the scope of this study are required to fully understand the microstructural evolution of the geopolymer gel phase.

Composition M2 showed the biggest microstructural differences compared to the other four compositions. The particular microstructural features of M2 were shown in Figure 4.7 and Figure 4.8. This sample was characterised by a very inhomogeneous microstructure with the presence of several secondary phases in addition to a geopolymer-like gel phase. Despite the inhomogeneous microstructure, $\mathrm{x}$-ray diffraction analysis revealed a largely amorphous material with a diffraction pattern very similar to the other compositions. The only secondary crystalline phase that was identified, except for the presence of crystalline quartz and cristobalite impurities from the metahalloysite, was small amounts of trona. Since the flower-like needle and platelet structures shown in Figure 4.7(e) were the only phases that seemed to have a crystalline appearance, these structures were attributed to the trona phase. The two main phases shown in Figure 4.7(a) - (d) were believed to be some sort of amorphous sodium-rich, silicate-dominated phase. However, the reason for the morphological difference between the two chemically similar phases is unknown. The reason for the significant difference between the microstructure of the sample of composition M2 and the other compositions is also unclear. Initially, the higher amount of sodium silicate solution compared to M1, M3 and M4 was believed to be responsible for these microstructural peculiarities. However, the same features were not observed in the sample of composition M5 which used an even higher amount of sodium silicate solution. 
The results of the ${ }^{27} \mathrm{Al}$ and ${ }^{29} \mathrm{Si}$ MAS-NMR analysis were presented in Figure 4.10. Both, the ${ }^{27} \mathrm{Al}$ and ${ }^{29} \mathrm{Si}$ spectra of compositions M1, M2, M4 and M5 were almost identical. Only composition M3 showed some notable differences. Despite the presence of some inhomogeneities in the geopolymer structures, the almost exclusive tetrahedral coordination of the aluminium atoms in all compositions was evident from the ${ }^{27} \mathrm{Al}$ spectra. In combination with the XRD results, confirming the largely $\mathrm{X}$ ray amorphous nature of all five compositions, these results clearly suggest the conclusion that all compositions formed well-reacted geopolymers. The small resonances at $\sim 3 \mathrm{ppm}$ can be attributed to trace amounts of residual unreacted metahalloysite present in the partially reacted areas. This is consistent the SEM observation discussed above which identified small areas of only partially reacted agglomerates of metahalloysite particles. The additional minor resonance of the M3 sample at $28 \mathrm{ppm}$ can be attributed to very small amounts of aluminium in V-fold coordination and supports the assumption that was made before based on the microstructural appearance that composition M3 showed a slightly lower degree of reaction, probably due to the insufficient amount of alkali cations. The main resonance at around $-88 \mathrm{ppm}$ that was observed for all five compositions in the ${ }^{29} \mathrm{Si}$ MAS-NMR spectra can be associated with the presence of predominantly $\mathrm{Q}_{4}(4 \mathrm{Al})$ structural units where each site of the tetrahedral silicon atom is connected to an aluminium atom via an oxygen bridge. Although other silica species were likely to be present in all samples due to the relative broadness of the main resonance, the comparatively larger width of the peak for composition M3 indicated the presence of a wider range of silica species with different coordination states for this sample. The resonance at the shoulder of the main peak at around - 106 ppm could be attributed to silica units without any surrounding alumina sites $\left(\mathrm{Q}_{4}(0 \mathrm{Al})\right)$ and most likely arose from the crystalline $\mathrm{SiO}_{2}$ impurities of the metahalloysite, i.e. quartz and cristobalite, which were present in all samples.

The $\mathrm{x}$-ray diffraction analysis confirmed the generally amorphous nature of all samples, as shown in Figure 4.9. The wider amorphous hump of the XRD diffractogram of composition M3 compared to the other compositions indicated a somewhat different and less structured nature of the amorphous phase. This is in 
agreement with the SEM and NMR results. As mentioned before, the quartz and cristobalite phases found in all samples were the result of unreactive crystalline impurities in the metahalloysite. Compositions M2 and, to a lesser extent, M4 were the only samples that showed the presence of an additional crystalline phase which was identified as trona. However, it is not clear if the trona phase actually formed as a secondary phase in the geopolymer structure or if it only formed on the fractured surface. Since the ground XRD samples were stored in sealed glass vials for several weeks prior to their analysis, the crystallisation reaction could have occurred on the exposed particle surface during that time. Likewise, the trona crystals could have formed on the exposed fracture surfaces of the bar specimens prior to their analysis under the SEM. However, this aspect was not investigated in any more detail.

\subsection{Thermal behaviour}

\subsubsection{Microstructural evolution and crystallisation}

The microstructural evolution and crystallisation behaviour of the two matrix compositions used for the fabrication of composites, M1 and M5, was investigated on bar pieces heated to temperatures between 500 and $1000^{\circ} \mathrm{C}$. The SEM analysis of the fracture surfaces revealed little change compared to the microstructures observed for unheated samples of the same composition for temperatures up to $800^{\circ} \mathrm{C}$. Although some shrinkage/densification could be observed on a macroscopic level, the SEM analysis did not indicate any significant change in the appearance of the microstructure. However, the formation of small rod-like shapes was observed in some areas of the $\mathrm{M} 1-600^{\circ} \mathrm{C}, \mathrm{M} 1-800^{\circ} \mathrm{C}$ and $\mathrm{M} 5-600^{\circ} \mathrm{C}$ samples, as shown in Figure 4.11 for sample $\mathrm{M} 1-600^{\circ} \mathrm{C}$. Attempts to determine the chemical composition of these rods by EDS mapping and spot analyses, however, were unsuccessful. 


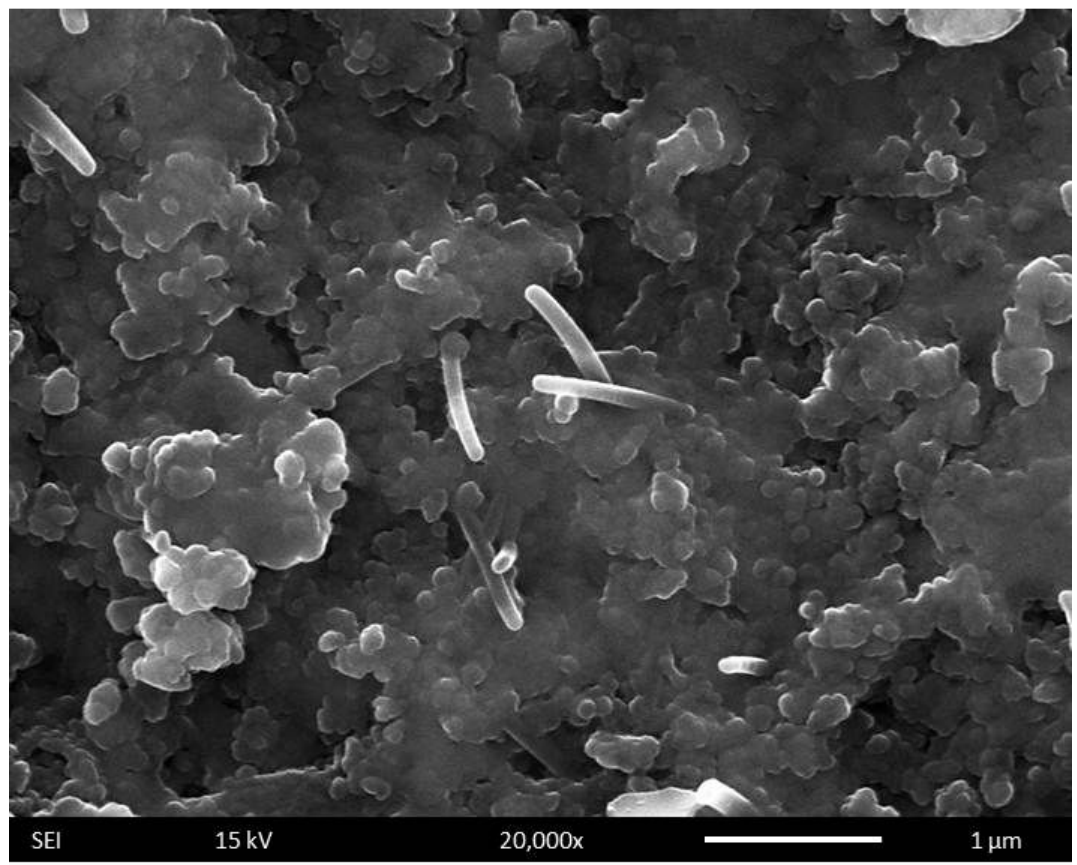

Figure 4.11: Microstructure and formation of small rod-like shapes in sample $\mathrm{M} 1-600^{\circ} \mathrm{C}$
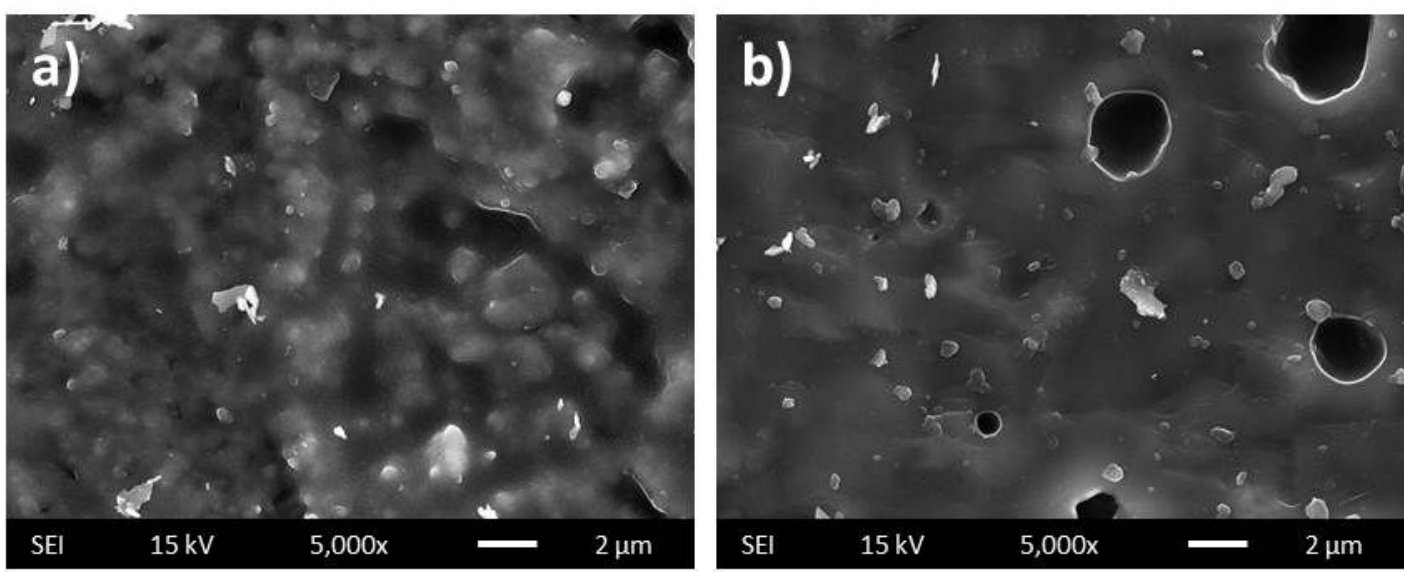

Figure 4.12: Microstructural appearance of samples M1 (a) and M5 (b) after heating to $1000^{\circ} \mathrm{C}$

The microstructural appearance of both compositions changed significantly after heating to $1000^{\circ} \mathrm{C}$, see Figure 4.12 . At closer level, the structure of the M5 sample appeared denser and more homogeneous compared to the M1 sample. The latter was characterised by what appeared to be small particles or crystals embedded in a glassy phase. Whereas the structure shown in Figure 4.12(a) was representative for large parts of the surface of sample M1, the M5 sample was characterised by a very inhomogeneous, porous structure as shown in Figure 4.13. Interestingly, however, the accumulation of a large number of small pores in $\sim 5-10 \mu \mathrm{m}$ wide rings around 
a much denser centre area, as can be seen in Figure 4.13, was also observed in some areas of the M1 sample.

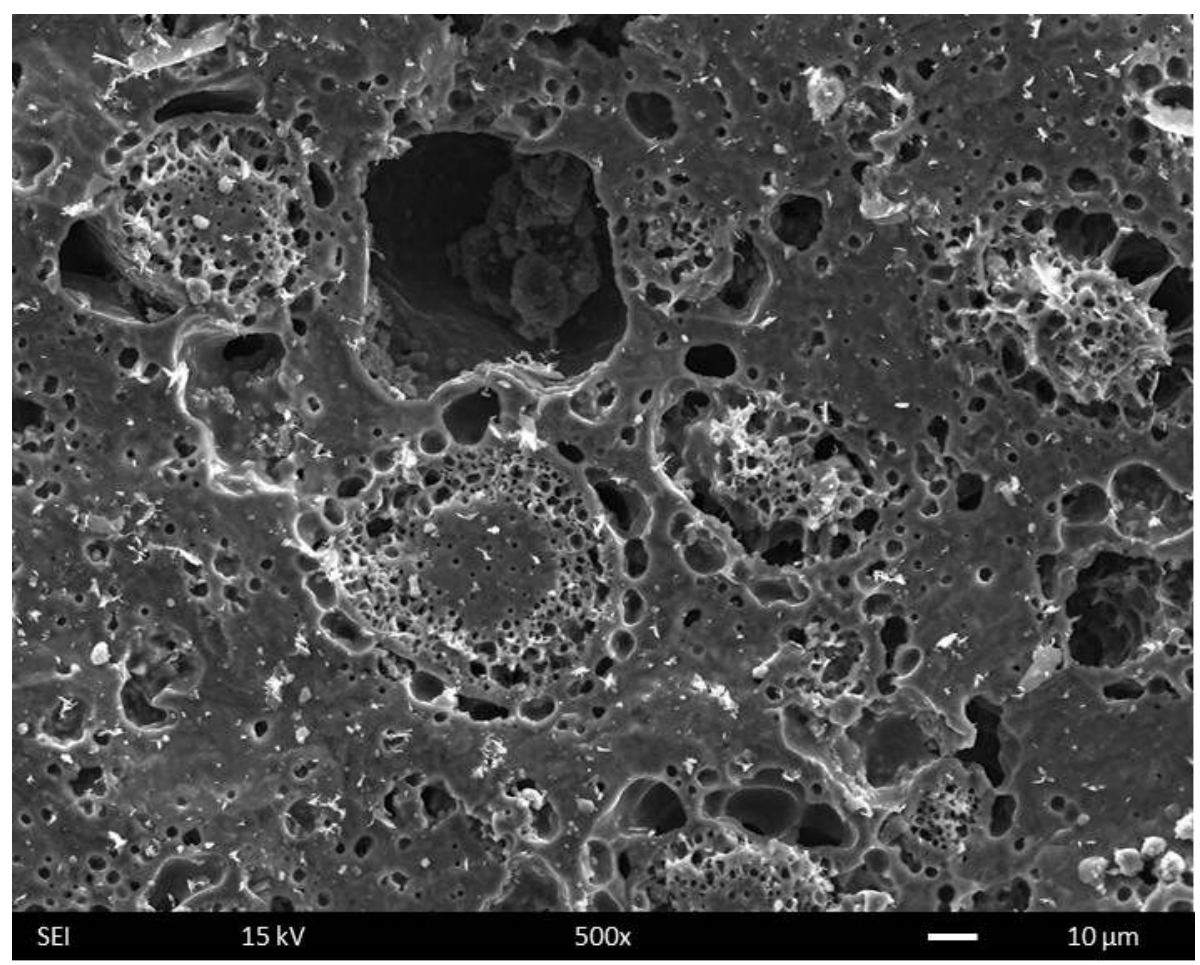

Figure 4.13: Low resolution SEM image of sample $\mathrm{M} 5-1000^{\circ} \mathrm{C}$ revealing a very inhomogeneous and porous structure

Macroscopically, all samples were subject to cracking. However, sintering or melting across the crack surfaces, thereby closing the initial cracks was clearly visible for the samples heated to $1000^{\circ} \mathrm{C}$. Although this effect was not analysed in further detail, it should be noted that the crack surfaces and the bulk of the sample showed slightly different microstructural appearance. Significant deformation of the bar pieces with increasing temperature was also observed. In all cases, the deformation was characterised by bending of the bars towards the bottom surface of the asmanufactured sample irrespective of the fact on which surface, i.e. sides, top or bottom, the bars were positioned in the furnace.

Typical diffractograms of powdered samples are shown in Figure 4.14. The diffraction patterns for the two different compositions were essentially identical for samples heated to 500,600 and $1000^{\circ} \mathrm{C}$. However, dissimilar behaviour was 
observed at $800^{\circ} \mathrm{C}$. Compared to the unheated geopolymer, a broadening and flattening of the amorphous hump was observed after heating to $500^{\circ} \mathrm{C}$. The quartz and cristobalite peaks remained largely unchanged. Also, weak but clearly identifiable diffraction peaks corresponding to trona were observed in the heated samples. Heating to $600^{\circ} \mathrm{C}$ resulted in no noticeable change of the diffraction pattern.

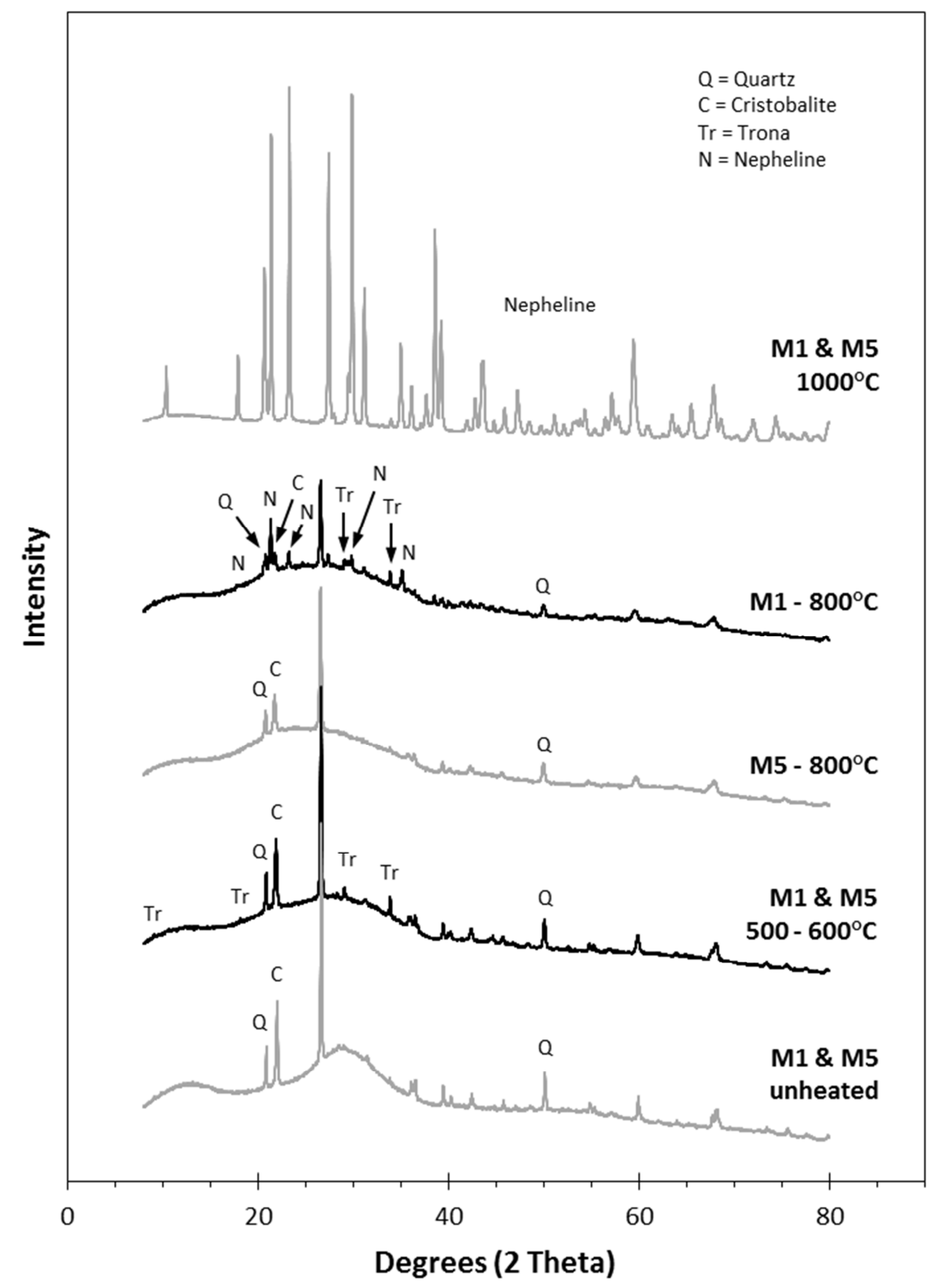

Quartz (PDF 01-089-8935) Cristobalite (PDF 04-007-2134)

Trona (PDF 00-029-1447) Nepheline (PDF 00-035-0424)

Figure 4.14: $\mathrm{X}$-ray diffraction patterns for compositions $\mathrm{M} 1$ and $\mathrm{M} 5$ after heating up to $1000^{\circ} \mathrm{C}$ 
At $800^{\circ} \mathrm{C}$, a slight change in the appearance of the amorphous hump as well as a decrease of the peak intensity of the quartz and cristobalite impurities was observed for both compositions. The decrease in the amount of the quartz and in particular cristobalite appeared to be more significant for composition M1 than for M5. Whereas quartz and cristobalite were the only crystalline phases present in the M5 sample after heating to $800^{\circ} \mathrm{C}$, composition $\mathrm{M} 1$ also showed several other weak diffraction peaks which could be attributed to trona and nepheline $\left(\mathrm{NaAlSiO}_{4}\right)$. Heating to $1000^{\circ} \mathrm{C}$ resulted in the nearly complete transformation into nepheline of both compositions. Except for possible trace amounts of quartz and cristobalite, no other crystalline phases were detected.

\subsubsection{Thermal analysis}

A combined TGA/DSC measurement of composition M1 was carried out to obtain some information about the thermal behaviour of this geopolymer. The results are presented in Figure 4.15. The geopolymer showed a weightloss of $\sim 22 \%$ which can be attributed to the loss of water that is mostly present in the pores of the geopolymer network. The majority of the weight loss occurred below $250-300^{\circ} \mathrm{C}$. Above $700^{\circ} \mathrm{C}$, no further weight change was observed. The DSC curve showed a large

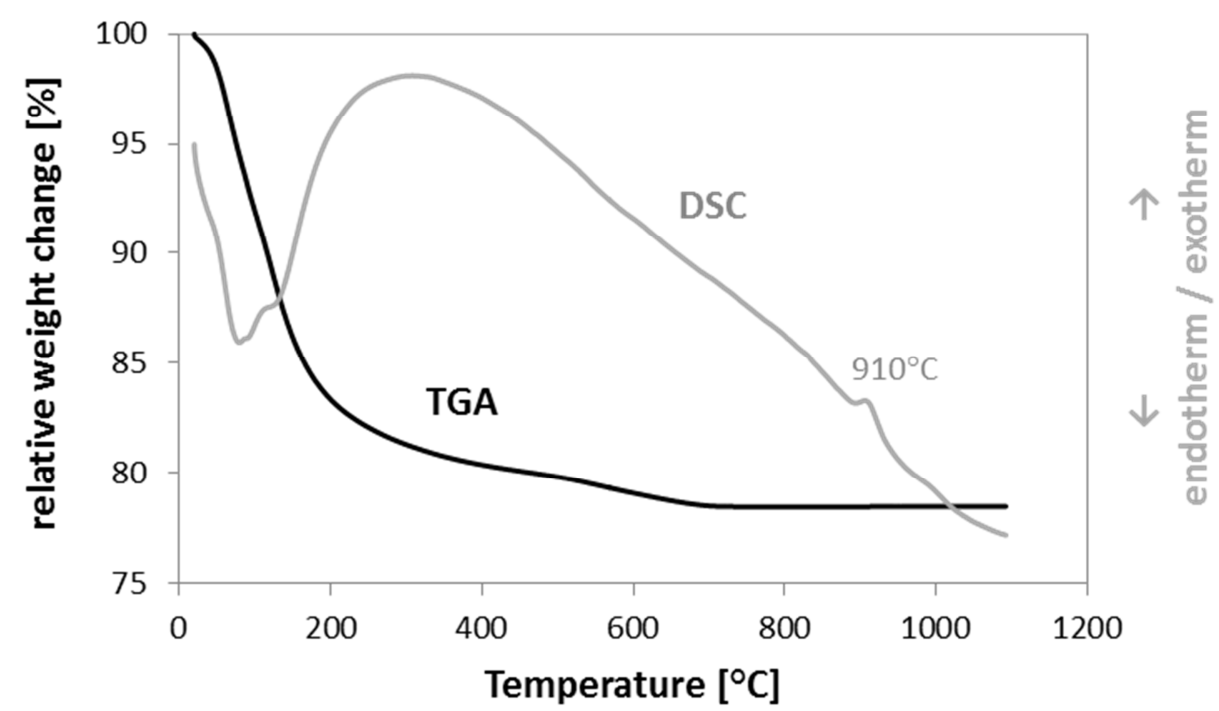

Figure 4.15: Weighloss and DSC curves for composition M1 
endothermic peak between 20 and $250^{\circ} \mathrm{C}$, consistent with the main loss of water. The only other feature of the DSC curve was a small exothermic peak at around $910^{\circ} \mathrm{C}$. This peak can be attributed to the crystallisation of the geopolymer into nepheline.

\subsubsection{Discussion}

Although sample cracking and some densification could be observed on a macroscopic scale, the SEM analysis of the heated samples of composition M1 and M5 showed no major changes in the microstructural appearance of both compositions up to $800^{\circ} \mathrm{C}$. However, the change in the general appearance of the amorphous hump in the XRD diffractograms compared to the unheated diffraction pattern indicates some structural changes within the amorphous phase (see Figure 4.14). Up to a temperature of $600^{\circ} \mathrm{C}$, both compositions showed identical $\mathrm{x}$-ray diffraction behaviour. However, the detection of trona in both composition after heat treatment at 500 and $600^{\circ} \mathrm{C}$ was surprising because it was not detected in either of the unheated compositions. Also, trona is a hydrated sodium hydrogen carbonate and dissociates at higher temperature, possibly forming sodium carbonate or other sodium oxide compounds. However, no suitable match was found for related Nacompounds. Thus, it can only be speculated that the trona phase forms either during the cooling period or during the relatively long storage period of the ground samples (the ground samples were stored in sealed glass vials between 4 to 10 weeks before XRD analysis). It may be possible that free sodium ions that may be present in the geopolymer, carbonate and re-hydrate in the apparent environment over time, thus, forming the trona phase. In any case, further investigations would be required to clarify the role of trona in the heated geopolymers.

While the samples of composition M1 and M5 heated to $1000^{\circ} \mathrm{C}$ both showed essentially identical diffraction patterns with the nearly complete transformation of the amorphous phase into crystalline nepheline, the comparison of the XRD patterns of the $800^{\circ} \mathrm{C}$ samples indicates a difference in the phase evolution of the two compositions between 600 and $1000^{\circ} \mathrm{C}$ (see Figure 4.14). At $800^{\circ} \mathrm{C}$, the crystallisation of nepheline in composition M1 has already started. The lower crystallisation temperature of composition M1 may be attributed to the higher 
sodium content compared to composition M5. However, these observations are not consistent with the DSC measurement which indicated the crystallisation of the matrix to occur around $910^{\circ}$

\subsection{Discussion on the reproducibility of mechanical strength with regard to fabrication process and microstructure}

In the previous section, the results of the geopolymer matrix investigations were presented and various aspects of the fabrication process, the mechanical properties and the microstructure were discussed individually. In this section, all of these results are taken into consideration and are discussed collectively in order to find a possible explanation for the apparent issue of the reproducibility of the mechanical properties.

It appears contradictory that the considerable efforts that were put into controlling the mixing process and optimising the fabrication process, which clearly resulted in an improved sample quality, did not reflect in a more consistent reproducibility of the mechanical strength. Although some sample series achieved excellent strength results, giving a closer indication of the true properties inherent to the respective composition, other sample series of the same composition achieved significantly lower strengths. These strength variations appeared to be random and unpredictable. Because the strength variation within each sample series, regardless if it achieved a high or low average strength, was generally reasonably good and significant variations were only observed between different sample series, it is believed that this issue was most likely in some way connected to the general processing and manufacturing processes. However, no obvious sources of error could be identified in the apparent processes that would account for the extremely inconsistent mechanical performance of the specimens. Although the processing and manufacturing method that was used in this study was by no means optimal and minor variations of the processing parameters and conditions (e.g varying room temperature, etc.) were unavoidable, they were believed to offer reasonably good control- and repeatability. The mechanical mixing was also a major improvement 
compared to the initial hand-mixing and in any case provided a significantly higher degree of homogeneity and, thus, reproducibility of the process and quality of geopolymer binder. While a high-shear mixer would most likely provide a better homogenisation than the mixing process used here, there are many different mixing processes that have been reported in the literature for the processing of geopolymers. However, none of them state any issues with the reproducibility of the mixing result. While the type of mixing process certainly has some effect on the properties of the geopolymer binder and the resulting geopolymer, it is not apparent why the result of the present mixing process should be inferior to most of these other mixing methods. Therefore, if the particular mixing process was responsible for the inconsistent reproducibility, it seems likely that similar problems would have also been encountered with other types of mixing processes before, too. The fact that very good results could be achieved from the same process also suggests that the general mixing process was not the key problem. Since the general processing and fabrication of the geopolymer samples in this study was otherwise not majorly different from what has been described in the current literature, the source of the problem remains largely unclear. It can only be hypothesised that the inability of adequate reproduction of the mechanical strength may arises from the accumulation of several minor inconsistencies throughout the fabrication process despite best efforts to control each parameter. In any case, it can be concluded that the technological processing and fabrication of high quality geopolymers along with a high degree of reproducibility seems much more difficult than suggested by the existing literature.

The presence of some inhomogeneities in all geopolymer compositions was evident from the microstructural analysis. This indicates that the standard mixing process with five minute homogenisation time did not achieve full homogenisation of the geopolymer binder. It was concluded in a previous section that these inhomogeneities were only partially reacted metahalloysite agglomerates. These partially reacted inhomogeneities were characterised by a comparatively higher aluminium and lower sodium content compared to the main geopolymer phase and displayed a microstructure of small discrete geopolymer-like gel particles similar to 
the microstructure that was observed for composition M3. The position of these not fully reacted areas corresponds with the position of slightly lowered and elevated areas that contribute to the roughness of the fracture surface as was shown in Figure 4.6. This can be explained when imagining the partly reacted metahalloysite agglomerates acting in a similar way to particle fillers during sample failure. However, unlike with particle fillers which are generally stronger than the surrounding matrix, these partially reacted agglomerates are softer and weaker than the main geopolymer phase and act as defects in the material. Consequently, even higher strength should be achieved for fully reacted mixtures of the same compositions. This may be achieved by better mixing and additional milling or other treatment of the dehydroxylated halloysite may be able to achieve better homogeneity. In that regard, the supposedly better homogenisation of the binder for the longer mixed sample batches should also have resulted in a higher strength and better reproducibility. However, no improvement of the reproducibility issues was observed and only a small number of compressive samples indicated higher strength after longer mixing times. In any case, the fact that the standard mixing process resulted in an incomplete homogenisation of the binder does not explain the strength variation between the different sample series since the mixing and fabrication process was the same for all samples. Thus, even if the inhomogeneities reduce the strength of the composition, this should be consistent for all sample batches.

The possibility of other defects such as large pores can be largely excluded as the examination of the fracture surfaces did not reveal any such evidence. Since no other obvious explanation for the observed strength variability was found, it is suggested that the comparatively low strength of some sample series is the result of the presence of spatially localised composition gradients or other inhomogeneities (other than the partially reacted metahalloysite agglomerates) in the specimens. These inhomogeneities act as a weak link in the geopolymer bulk material and are distributed across the sample. This would explain why the majority of the weaker samples did not fail at the point of maximum stress concentration but at a lower stress level along the support span. The extreme strength variation observed in some cases between the three measurements of the same bar (100 and $40 \mathrm{~mm}$ spans) would 
support the assumption that these defects are spatially localised within the sample. However, it is largely unclear what caused or affected the formation of these defects. Because significant strength variation was mainly observed between different sample batches as opposed to within the same batch of samples, it seems likely that the defects were introduced during the mixing and processing stage of making the geopolymer specimens. It can only be assumed that the absence of similar weak spots in the high strength sample batches was the result of the coincidental conjunction of all relevant processing parameters for these batches. Nevertheless, other possibilities besides a purely technological explanation such as local de-mixing of components or an inconsistent geopolymerisation reaction within the samples should also be considered. However, the clear identification of the nature and origin of these defects requires a careful microstructural analysis that was beyond the scope of this study. 


\section{Chapter 5}

\section{Mechanical properties of unidirectional composites}

Several parameters and their effects on the flexural strength, elastic modulus and general failure behaviour of unidirectional fibre reinforced geopolymer matrix composites were investigated including fibre type, fibre sizing, matrix strength and drying time. The influence of varying specimen dimensions and span-to-depth ratio was also examined. The results are presented in the following section, followed by a discussion at the end of this chapter. Unless stated otherwise, the following standard parameters applied to all specimens:

- Matrix composition: M1 (mechanically mixed)

- Specimen dimensions: approximately $10 \times 3 \times 115 \mathrm{~mm}$

- Fibre volume content: approximately $30 \%$

- Number of samples per sample series: five

- Curing: $40^{\circ} \mathrm{C}$ and $48 \mathrm{~h}$ (sealed)

- Drying time before testing: seven days (ambient conditions)

- Test method: Three-point bending

- Testing span: $100 \mathrm{~mm}$ 


\subsection{Effects of fibre type and fabrication process on the flexural properties and stress-strain behaviour}

\subsubsection{General observations and fabrication process}

Over the course of this study, the ultimate flexural strength of all types of composites was generally found to be subject to a very large variability. To some degree, this does not come as a surprise and a relatively high scatter of the results was to be expected from an all-manual fabrication process. However, a closer analysis of the results revealed that the strength variation within each type of composite was not random but followed a general trend irrespective of the fibre type or any other parameter. For a better understanding of the subsequent results, this characteristic is briefly discussed below using the example of three different composite types made from basalt, carbon and Nextel 610 alumina fibres.

The flexural properties of the three different sample series are displayed in Table 5.1. The table shows the variability between individual samples of each test series in regards to height and fibre content. It also shows that the highest flexural strength and elastic modulus do not necessarily correspond with the highest fibre content. In fact, the flexural strength appears to be less affected by the fibre content (within the limits displayed in Table 5.1, i.e. approximately $30 \pm 5 \%$ ) but rather correlates to the serial number of each sample series. The serial number represents the order in which the samples were fabricated. As outlined in the experimental section, only three samples could be fabricated from one batch of geopolymer binder. Thus, samples one and three are the first and last fabricated samples of batch one and samples four and five were generally fabricated in a second batch. Keeping this in mind, a clear trend of increasing strength from sample number one to three and four to five, respectively, can be observed for each sample series in Table 5.1. Therefore, the fabrication process appears to have a significant effect on the flexural strength of the composites. The only variable in the fabrication process between the samples in each batch is the mixing time of the geopolymer binder. This indicates that the strength of the sample is strongly affected by the mixing time of the geopolymer binder. The elastic modulus, however, does not follow the same pattern as the flexural strength. 
This finding is not restricted to the three composite types shown in Table 5.1 but was also observed for all other types of composites, irrespective of any of the investigated parameters. The few exceptions from this pattern such as sample M1-N610-2 are believed to be statistical variations due to inherent manufacturing defects that interfere with the general effect of the fabrication process on the composite strength. The corresponding stress-strain curves, displayed in Figure 5.1, show that the fabrication process, for the most part, only affects the ultimate strength. Although there is some variation in the behaviour of the individual curves, the general stressstrain response is roughly similar for all samples within a series.

Table 5.1: Flexural properties datasets of three exemplary composite types (M1-Basalt, M1-Carbon, and M1-N610) to show the general influence of the order of fabrication on the flexural strength

\begin{tabular}{|c|c|c|c|c|c|c|c|}
\hline & Sample & $\begin{array}{l}V_{\text {fibre }} \\
{[\%]}\end{array}$ & $\begin{array}{c}\mathbf{b} \\
{[\mathrm{mm}]}\end{array}$ & $\begin{array}{c}\mathbf{h} \\
{[\mathrm{mm}]}\end{array}$ & $\begin{array}{c}\sigma_{\mathbf{f}, \max } \\
{[\mathbf{M P a}]}\end{array}$ & $\begin{array}{c}\mathbf{E}_{\mathbf{f}} \\
{[\mathbf{G P a}]}\end{array}$ & $\begin{array}{c}\boldsymbol{\varepsilon}_{\mathbf{f}} \\
{[\%]}\end{array}$ \\
\hline \multirow{7}{*}{ 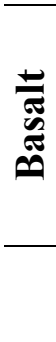 } & M1-B-1 & 25 & 10.01 & 3.46 & 128.26 & 27.60 & 0.63 \\
\hline & M1-B-2 & 32 & 10.04 & 2.68 & 143.07 & 32.05 & 0.54 \\
\hline & M1-B-3 & 29 & 9.98 & 3.00 & 193.80 & 29.64 & 0.75 \\
\hline & M1-B-4 & 27 & 10.02 & 3.16 & 156.36 & 29.49 & 0.67 \\
\hline & M1-B-5 & 33 & 10.03 & 2.64 & 169.41 & 36.75 & 0.57 \\
\hline & & & & mean & 158.18 & 31.10 & 0.63 \\
\hline & & & & stdev & 25.11 & 3.53 & 0.08 \\
\hline \multirow{7}{*}{ 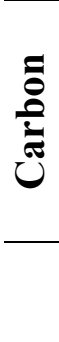 } & M1-C-1 & 32 & 10.05 & 2.84 & 108.05 & 52.46 & 0.21 \\
\hline & M1-C-2 & 35 & 10.07 & 2.62 & 142.92 & 66.44 & 0.22 \\
\hline & M1-C-3 & 29 & 9.92 & 3.22 & 170.54 & 56.27 & 0.39 \\
\hline & M1-C-4 & 32 & 9.99 & 2.84 & 143.85 & 53.21 & 0.39 \\
\hline & M1-C-5 & 33 & 9.89 & 2.82 & 179.05 & 68.22 & 0.26 \\
\hline & & & & mean & 148.88 & 59.32 & 0.29 \\
\hline & & & & stdev & 27.87 & 7.47 & 0.09 \\
\hline \multirow{7}{*}{$\begin{array}{l}\text { 음 } \\
0 \\
0 \\
0 \\
0\end{array}$} & M1-N610-1 & 29 & 9.98 & 3.51 & 348.30 & 86.57 & 0.40 \\
\hline & M1-N610-2 & 29 & 10.07 & 3.51 & 299.57 & 99.92 & 0.30 \\
\hline & M1-N610-3 & 30 & 10.03 & 3.47 & 469.81 & 105.73 & 0.44 \\
\hline & M1-N610-4 & 32 & 10.04 & 3.22 & 292.64 & 114.47 & 0.26 \\
\hline & M1-N610-5 & 32 & 9.79 & 3.33 & 460.15 & 116.11 & 0.40 \\
\hline & & & & mean & 374.09 & 104.56 & 0.36 \\
\hline & & & & stdev & 85.76 & 12.02 & 0.08 \\
\hline
\end{tabular}



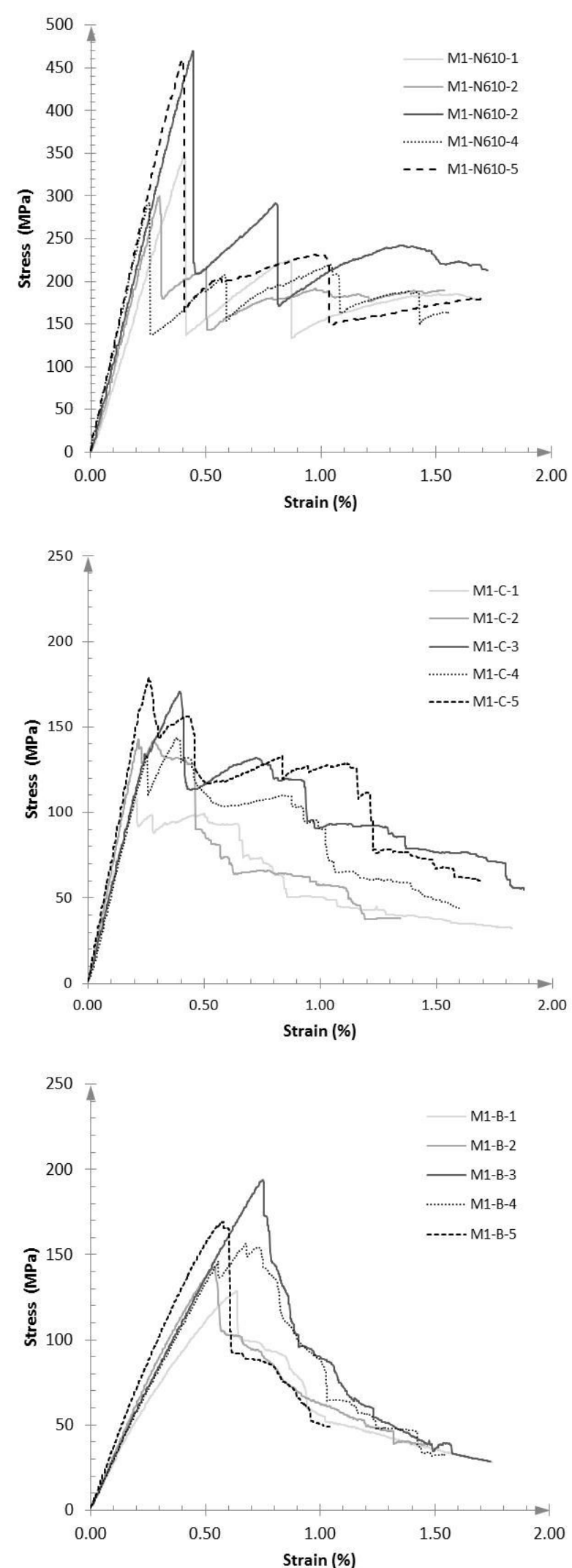

Figure 5.1: Stress-strain curves recorded for the alumina, carbon and basalt composites (top to bottom) presented in Table 5.1 
Due to the strong impact of the fabrication process on the flexural strength, the comparison between the different types of composites is somewhat difficult. The large variability of the flexural strength in each sample series causes a high uncertainty for the average strength values. The large variability also makes it difficult to clearly identify defective specimens that are weakened due to manufacturing defects and which may misrepresent the actual composite strength. In order to minimise the influence of the fabrication process, all samples of the same serial number would have to be individually compared with each other. However, this approach would be fairly lengthy and difficult to follow. Therefore, a combined approach was chosen to describe the results in the following section. For more clarity, the average flexural properties for each composite type will be used to compare the effects of the various parameters. Since the fabrication process should have equal effect on all types of composites the error that is associated with the average value should also be similar. Unless a sample can be clearly identified as defective, all samples of a series were included in the calculation of the average values. The stress-strain behaviour will be compared on the strongest samples of each series where appropriate. However, preference will be given to the stress-strain curves that represent the general failure behaviour of all specimens of a sample series the best. Therefore, all figures in the following sections should be considered in context with their corresponding tables.

\subsubsection{Fibre type}

Basalt, carbon and Nextel 610 alumina fibres were used to evaluate the effect of different fibre types on the flexural properties and failure behaviour of unidirectional fibre reinforced geopolymer matrix composites. The complete data sets for the three composite types were already presented in Table 5.1 and Figure 5.1 to explain the variability of strength within each sample series and the influence of the fabrication process. However, for reasons of clarity, the results are summarised again in Table 5.2 and Figure 5.2. It is clearly evident from Table 5.2 that the incorporation of all of these fibres drastically improves the mechanical properties of the geopolymer matrix. It can also be seen that the mechanical properties strongly depend on the fibre type. The basalt fibre composites show a slightly higher average flexural strength 
compared to the carbon fibres. An average flexural strength of $158 \mathrm{MPa}$ was obtained for basalt fibre composites with a minimum and maximum value of 128 and $194 \mathrm{MPa}$, respectively. The flexural strength of the carbon fibre composites varied between 108 and $179 \mathrm{MPa}$ with an average of $149 \mathrm{MPa}$. However, due to the large variability of the strength values the distinction between the two fibre types is of no statistical significance. Therefore, both composite types can be considered similarly strong. A clear difference between the two fibre types can be observed for the elastic modulus. The elastic modulus of the carbon fibre composites is about $60 \mathrm{MPa}$ and approximately twice the value of the basalt fibre composites. This can be attributed to the much higher modulus of the carbon fibre (240 GPa) compared to the basalt fibre $(89 \mathrm{GPa})$. The best mechanical properties were obtained with Nextel 610 alumina fibres. The flexural strength ranged between 293 and a maximum of 470 MPa with an average of $374 \mathrm{MPa}$. This corresponds roughly to a 2.5 times higher average flexural strength compared to basalt and carbon fibre composites at similar fibre contents. The average elastic modulus is $105 \mathrm{GPa}$. Again, the higher elastic modulus of these composites can be attributed to the higher modulus of the alumina fibres $(380 \mathrm{GPa})$.

Table 5.2: Effect of fibre type on the flexural properties of geopolymer matrix composites

\begin{tabular}{lcccc}
\hline Fibre type & $\begin{array}{c}\mathbf{V}_{\text {fibre }} \\
{[\mathbf{\%}]}\end{array}$ & $\begin{array}{c}\boldsymbol{\sigma}_{\text {f, } \max } \\
{[\mathbf{M P a}]}\end{array}$ & $\begin{array}{c}\mathbf{E}_{\mathbf{f}} \\
{[\mathbf{G P a}]}\end{array}$ & $\begin{array}{c}\mathbf{\varepsilon}_{\mathbf{f}} \\
{[\mathbf{\%}]}\end{array}$ \\
\hline Basalt & $29 \pm 3$ & $158 \pm 25$ & $31 \pm 4$ & $0.63 \pm 0.08$ \\
Carbon & $32 \pm 2$ & $149 \pm 28$ & $59 \pm 7$ & $0.29 \pm 0.09$ \\
Nextel 610 & $30 \pm 1$ & $374 \pm 86$ & $105 \pm 12$ & $0.36 \pm 0.08$ \\
\hline
\end{tabular}

Figure 5.2 shows that all three composite types fail in non-catastrophic fashion and can carry significant load after the initial failure occurred. Carbon and alumina fibre composites both show, for the most part, a linear elastic behaviour up to the point of failure. For the basalt fibre composites, a slight decrease in the slope of the stressstrain curve is observed at stresses above approximately $40-50 \mathrm{MPa}$. In some cases (see sample M1-B-1 in Figure 5.1) this effect was more pronounced than for others. All three composite types show a very distinct point of failure. A significant flattening of the stress-strain curves around the point of maximum stress associated 
with inelastic deformation is hardly evident. Beyond this point, all composites are characterised by a significant and relatively sudden decrease of the stress level. The carbon and alumina fibre composites show a step-like behaviour with increasing deflection. Between each step the stress level often increases again. Especially for alumina fibre composites the stress increase after each drop can be fairly significant. On the other hand, the step-like behaviour is less evident for the basalt fibre composites. These samples are typically characterised by a relatively sudden decrease from the maximum stress to an equal stress level of roughly $100 \mathrm{MPa}$. Subsequently, the stress-strain curves show a gradual flattening with increasing deflection.

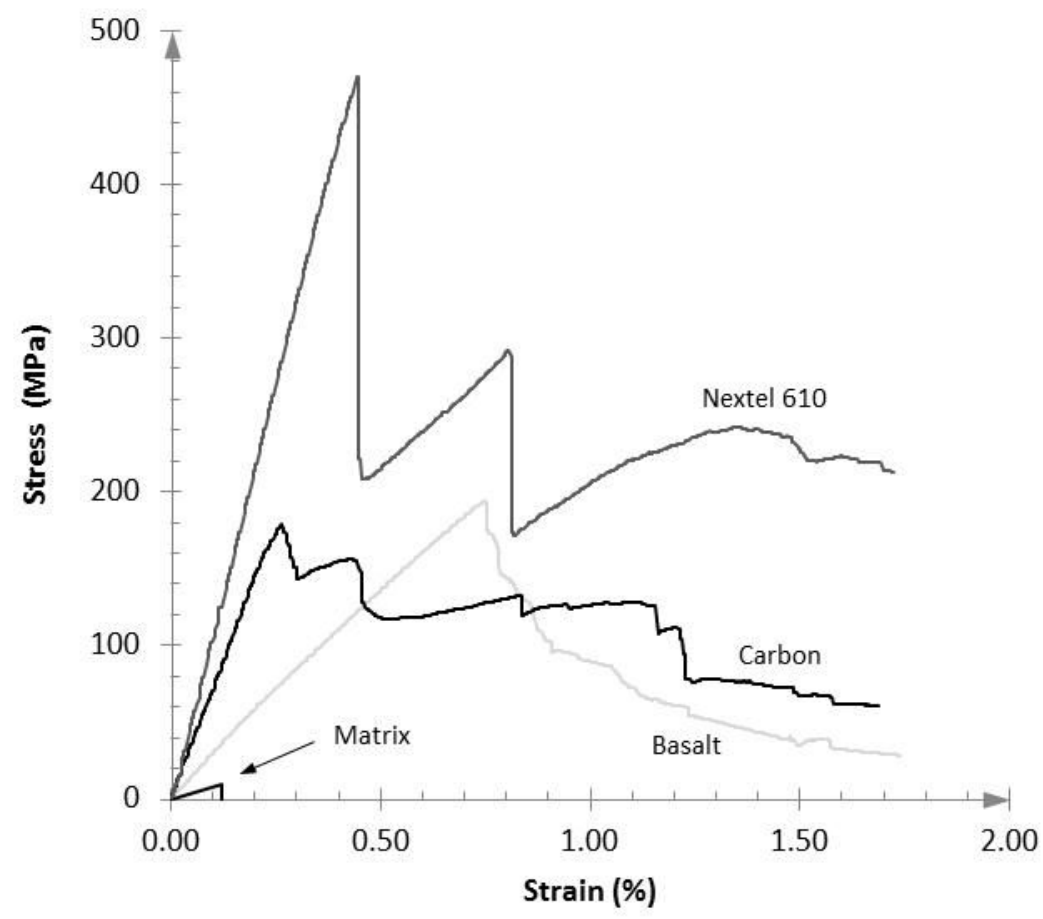

Figure 5.2: Typical stress-strain behaviour of unidirectional basalt, carbon and Nextel 610 alumina fibre reinforced geopolymer matrix composites

The typical macroscopic appearance of the fractured composites is presented in Figure 5.3. The basalt fibre composites were the only composite type that showed some extent of tensile failure and fibre fracture. The macroscopic failure was usually initiated by the formation of a clearly visible main crack on the tensile side around 
but not always at the point of central loading. After the initial crack formation, the crack generally deflected and spread horizontally to various degrees. Upon further loading the crack propagated further towards the compression surface without following any clear paths. In some cases the crack continued upwards in a nearly straight line with only small zigzag movement. In other cases a more diagonal crack propagation was observed often going along with additional delamination. However, it should be noted that neither the basalt nor any of the other composite types failed completely after flexural testing. The dominating failure mode for both carbon and alumina fibre composites was interlaminar shear. No tensile failure was observed for either of the two composite types. Figure 5.3 shows typical failure patterns for both
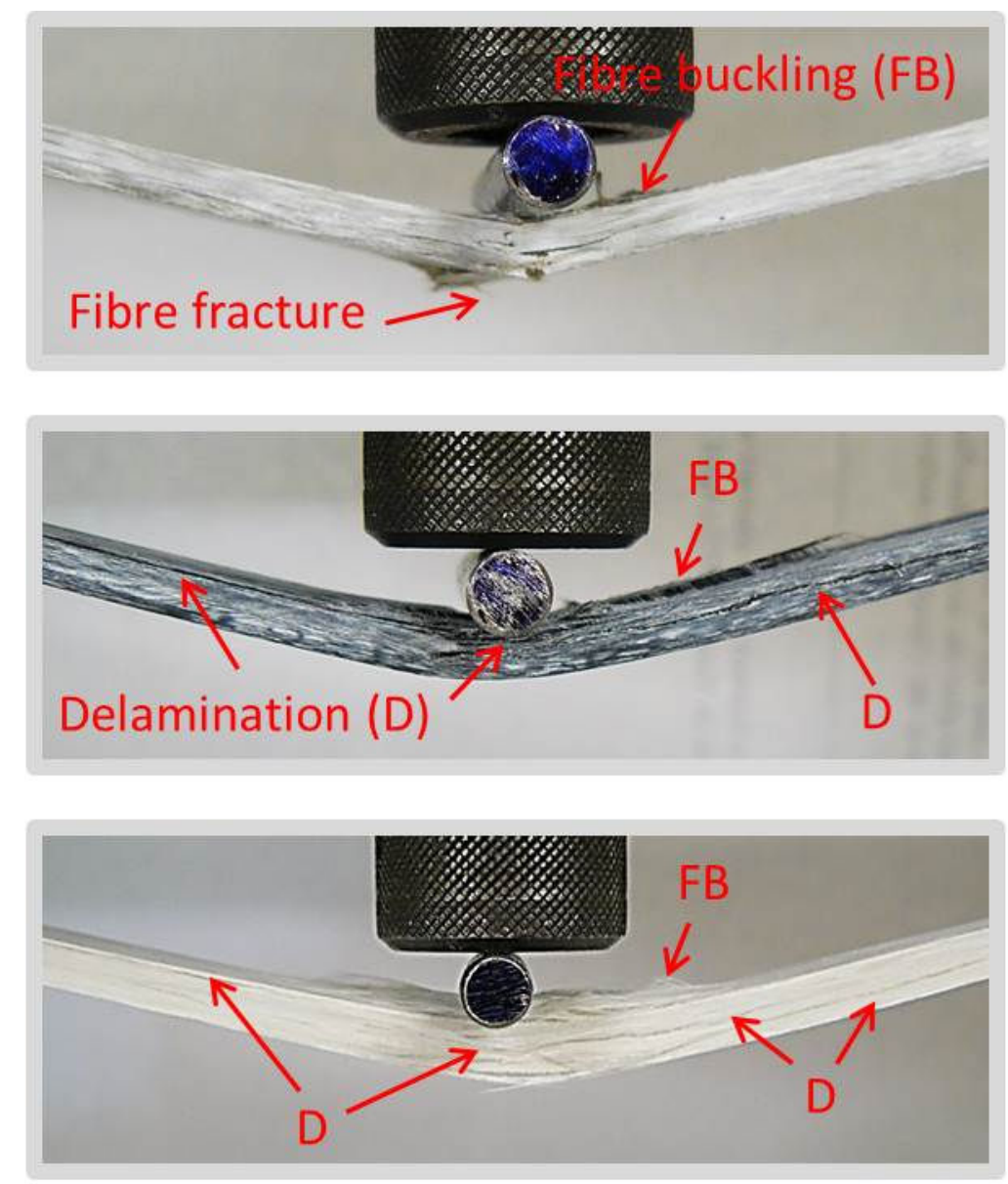

Figure 5.3: Typical macroscopic failure behaviour of UD composite bars made with basalt (top), carbon (middle) and Nextel 610 alumina fibres (bottom) 
types of composites. Several major delamination cracks can be identified that extent from the sample centre all the way to one of the sample ends. Various smaller delamination cracks can be observed around the central loading point. Fibre buckling on the compressive side was also observed for all three composite types to various degrees. The fibre buckling occurred at various times over the course of the flexural test. Some smaller decreases in the stress-strain curves before reaching the maximum stress may be attributed to fibre buckling. Fragmentation of the geopolymer matrix was observed at higher loads in particular with alumina and carbon fibre composites.

As described before, the calculated average flexural strength of the basalt and carbon fibre composites is relatively similar. This observation in combination with the comparatively low cost of the basalt fibre makes these composites very interesting from an economical point of view. However, the elastic modulus of the basalt fibre composites is comparatively low. Therefore, the combination of basalt with carbon and boron fibres was investigated in order to improve the stiffness of the basalt composites. The basalt-carbon composites were fabricated in the same way as basalt fibre composites but replacing four of the ten basalt fibre tapes with carbon tapes. The lay-up was symmetrical to the specimen centre in the order $B-C-B-C-B$. The fabrication of the basalt-boron composites was somewhat different. The boron fibres were available in form of a small sheet of parallel aligned monofilaments. The layers were cut to the appropriate length and about $10 \mathrm{~mm}$ width. Each of these layers consisted of roughly 50-60 boron monofilaments. However, the monofilament layers could not be impregnated and handled the same way as the multifilament fibre tapes. Therefore, the boron monofilament layer had to be placed in the mould first and then covered with geopolymer binder. The lay-up of the impregnated basalt fibre tapes followed as before. Three boron fibre layers were added in addition to the standard ten basalt fibre layers in the order $B-B$ or $-B-B-B-B-B$ or $-B-B-B-B-B o r-B$. The number of samples for this composite type was limited to three. 
Table 5.3: Flexural properties of mixed basalt/carbon and basalt/boron fibre reinforced geopolymer composites

\begin{tabular}{lcccc}
\hline Fibre type & $\begin{array}{c}\mathbf{V}_{\text {fibre }} \\
{[\mathbf{\%}]}\end{array}$ & $\begin{array}{c}\boldsymbol{\sigma}_{\text {f, } \max } \\
{[\mathbf{M P a}]}\end{array}$ & $\begin{array}{c}\mathbf{E}_{\mathbf{f}} \\
{[\mathbf{G P a}]}\end{array}$ & $\begin{array}{c}\mathbf{\varepsilon}_{\mathbf{f}} \\
{[\mathbf{\%}]}\end{array}$ \\
\hline Basalt/Carbon & $42 \pm 3$ & $125 \pm 44$ & $50 \pm 5$ & $0.26 \pm 0.05$ \\
Basalt/Boron & - & $189 \pm 12$ & $49 \pm 4$ & $0.40 \pm 0.08$ \\
\hline
\end{tabular}

As shown in Table 5.3 the average elastic modulus could be significantly improved by over $60 \%$ for both fibre combinations, resulting in an average modulus of about $50 \mathrm{MPa}$. However, the average strength of the basalt-carbon composites was lower than the ones with either of the fibre types taken separately. Only sample M1-BC-3 achieved a good strength of $197 \mathrm{MPa}$. This strength is similar to the maximum strength measured for the basalt-only composites. The other four samples showed comparatively low strength in the range of $82-124 \mathrm{MPa}$. This result is surprising because composites of either of the two fibre types taken separately achieved similar strength. The lower strength is also surprising considering that the fibre content of these samples was significantly higher. The higher fibre content was a result of a reduced composite thickness and was not intended. On the other hand, the basalt boron composites showed an improved strength compared to basalt fibre composites as well as a smaller standard deviation.

Different failure behaviour was observed for both types of composites compared to basalt fibre composites. Representative stress-strain curves are displayed in Figure 5.4. Both fibre combinations show linear elastic behaviour up to the maximum stress. Basalt-carbon composites show a step-like behaviour that in general resembles a combination of the stress-strain behaviour described before for carbon and alumina fibre composites. The basalt-boron composites all show two distinct steps before reaching a constant stress level with increasing deflection. In both cases, the main failure mode is delamination due to interlaminar shear. In some cases, fibre buckling on the compression side was also observed. Tensile failure as observed for the basaltonly composites was not evident. The delamination cracks extended from the centre to one of the ends of each specimen and generally occurred along one of the secondary fibre layers. 


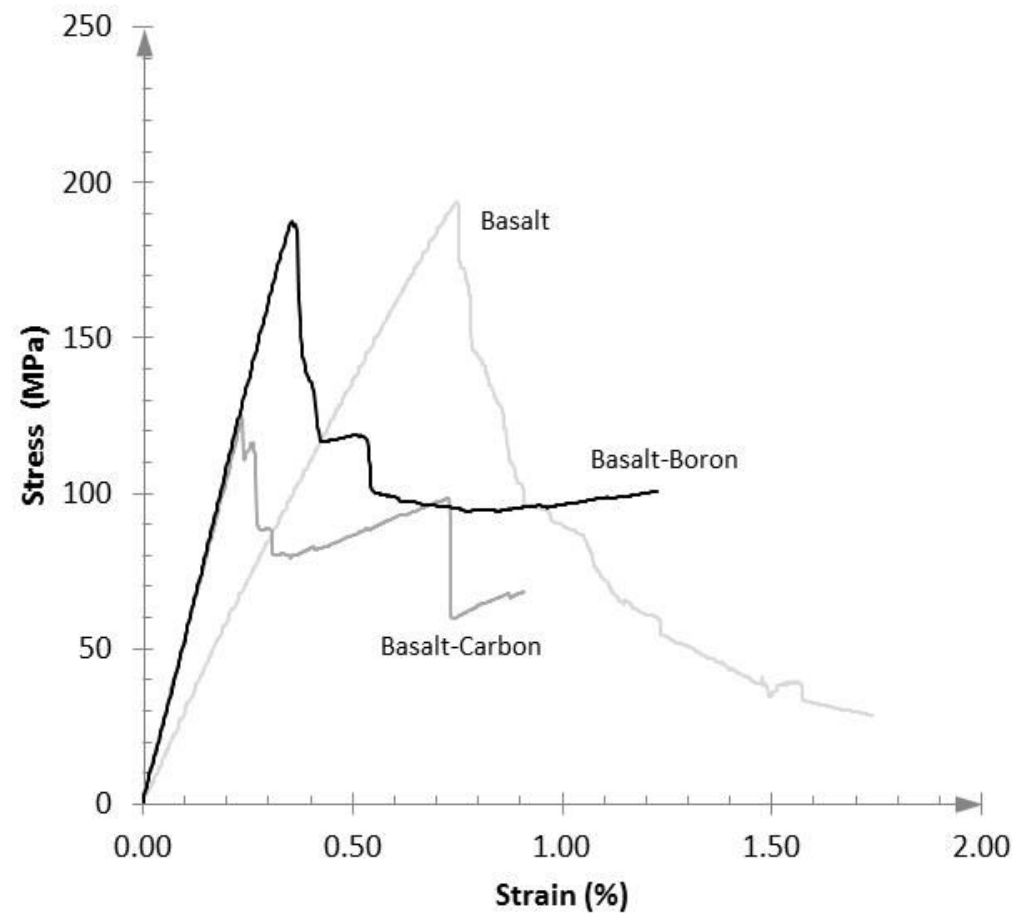

Figure 5.4: Representative stress-strain curves of basalt-carbon and basalt-boron fibre composites in comparison to basalt fibre composites. Note: The displayed curves only display the typical failure response of the different composites and do not accurately represent the relation between the average strength of the three composite types, see Table 5.2

\subsubsection{Fibre distribution in composite bars}

The typical distribution of fibres in the basalt, carbon and alumina fibre reinforced composite bars is shown in Figure 5.5. All three composite types showed an inhomogeneous microstructure with matrix-rich interlaminar regions between the fibre layers. No significant differences in the general fibre distribution were found between the different composite bars of a fabrication series or for the use of sized or desized fibre tapes. The impregnation of the individual fibre tapes was generally good even in densely packed areas, as can be seen in Figure 5.5(d). However, the apparent matrix-poor areas that can be observed within some of the individual fibre bundles in Figure 5.5(a) and (c) suggest that not all fibre tapes were fully impregnated. The darker colour of the matrix within the fibre layers of the carbon fibre samples arises from a smearing of the carbon fibres during the polishing process. The apparent cracks that can be observed in all three composite samples 
shown in Figure 5.5, particularly in the matrix-rich layers, are believed to be a result of the cutting, grinding and polishing of the samples and subsequent drying process.
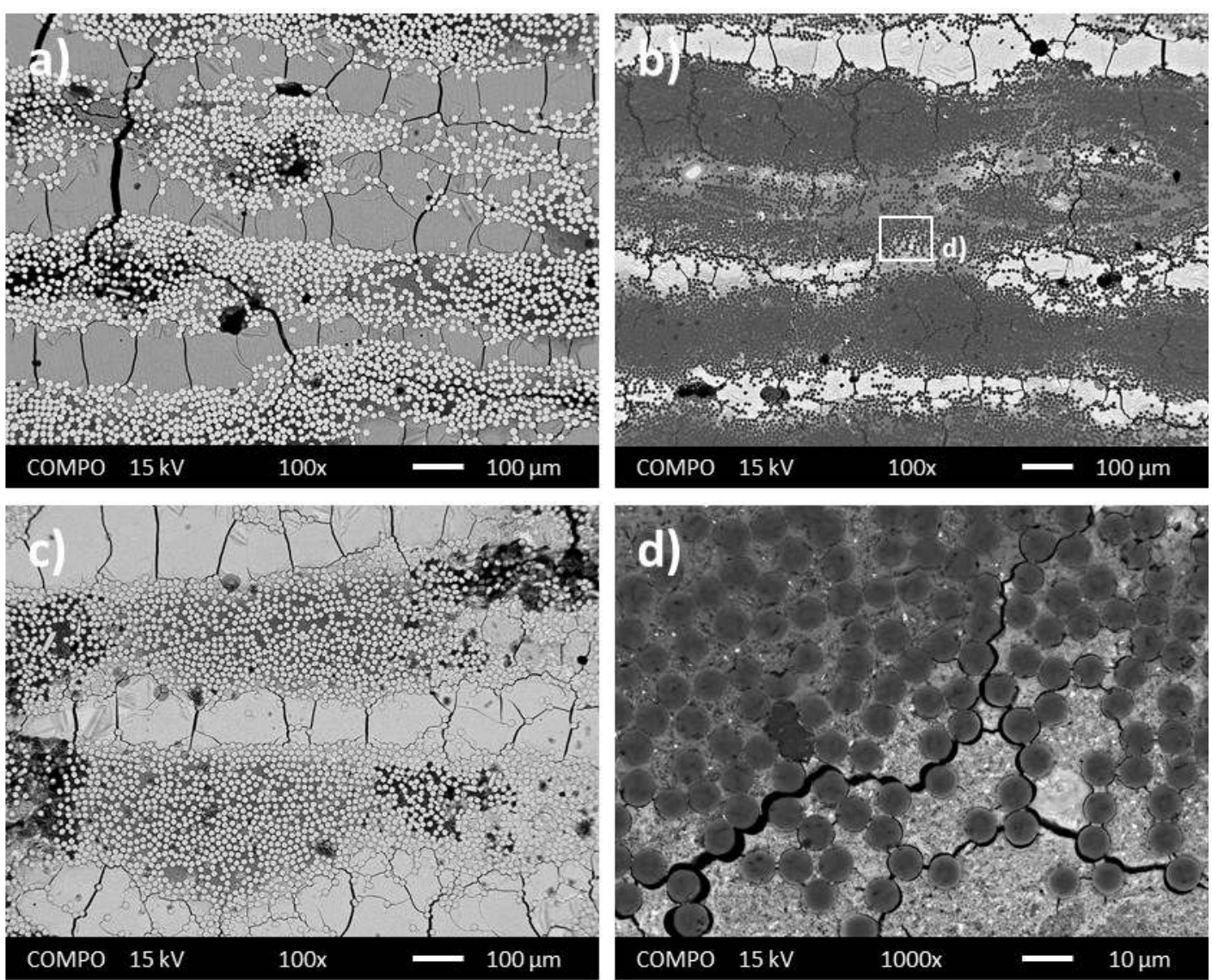

Figure 5.5: Typical fibre distribution in (a) basalt, (b, d) carbon and (c) Nextel 610 fibre composites

\subsection{Other factors influencing the flexural properties}

\subsubsection{Fibre sizing}

The influence of the fibre sizing was studied on carbon and Nextel 610 alumina fibres. The results are summarised in Table 5.4. With an average strength of 205 $\mathrm{MPa}$, composites made from desized carbon fibres were roughly one third stronger than composites made from sized carbon fibres. The higher average strength is accompanied by a higher deviation of the measured strengths with a minimum of 141 $\mathrm{MPa}$ and a maximum strength of $262 \mathrm{MPa}$. The average elastic modulus of $72 \mathrm{GPa}$ for the desized carbon fibre composites is also higher than the modulus of their sized 
counterparts. A slight difference in the impregnation behaviour of the sized and desized carbon fibre tapes was also noted. The desized fibre tapes could easily be impregnated but appeared to hold less geopolymer binder in between the individual fibres and therefore consistently resulted in a smaller sample height and higher fibre content, respectively.

Table 5.4: Effect of fibre sizing on the flexural properties of geopolymer matrix composites

\begin{tabular}{lcccc}
\hline Fibre type & $\begin{array}{c}\mathbf{V}_{\text {fibre }} \\
{[\mathbf{\%}]}\end{array}$ & $\begin{array}{c}\boldsymbol{\sigma}_{\mathbf{f}, \mathbf{m a x}} \\
{[\mathbf{M P a}]}\end{array}$ & $\begin{array}{c}\mathbf{E}_{\mathbf{f}} \\
{[\mathbf{G P a}]}\end{array}$ & $\begin{array}{c}\mathbf{\varepsilon}_{\mathbf{f}} \\
{[\mathbf{\%}]}\end{array}$ \\
\hline Carbon & $32 \pm 2$ & $149 \pm 28$ & $59 \pm 7$ & $0.29 \pm 0.09$ \\
Carbon-DS* & $37 \pm 1$ & $205 \pm 44$ & $72 \pm 9$ & $0.31 \pm 0.04$ \\
Nextel 610 & $30 \pm 1$ & $374 \pm 86$ & $105 \pm 12$ & $0.36 \pm 0.08$ \\
Nextel 610-DS* & $30 \pm 1$ & $358 \pm 77$ & $101 \pm 9$ & $0.38 \pm 0.03$ \\
\hline *DS = desized fibres & & & &
\end{tabular}

The average strength of the desized alumina fibre samples was somewhat lower than for the sized fibres but given the large standard deviation, no significant difference between the sized and desized fibres can be established. Except for sample M1N610-DS-1 which achieved the lowest strength of all alumina fibre composites with a value of $225 \mathrm{MPa}$, the remaining four samples in the series varied in a much smaller range between 365 and $419 \mathrm{MPa}$. Thus, it may be argued that this specimen is in fact defective and not representative of the material properties. In comparison, the sized fibre samples achieved higher absolute strength values with two samples in the range of $460-470 \mathrm{MPa}$. However, with two samples at around $295 \mathrm{MPa}$, they also resulted in two comparatively weak samples. The elastic modulus is similar for both sized and desized alumina fibre samples.

Irrespective of any possible influence of the fibre sizing on the composite strength, the fibre sizing proved to have very little effect on the general stress-strain and failure behaviour of the two composites. Typical stress-strain curves of sized and desized carbon and alumina fibre composites, respectively, are shown in Figure 5.6. Similarly to sized fibres composites, the desized fibre composites failed due to delamination and fibre buckling without any notable tensile failure. 


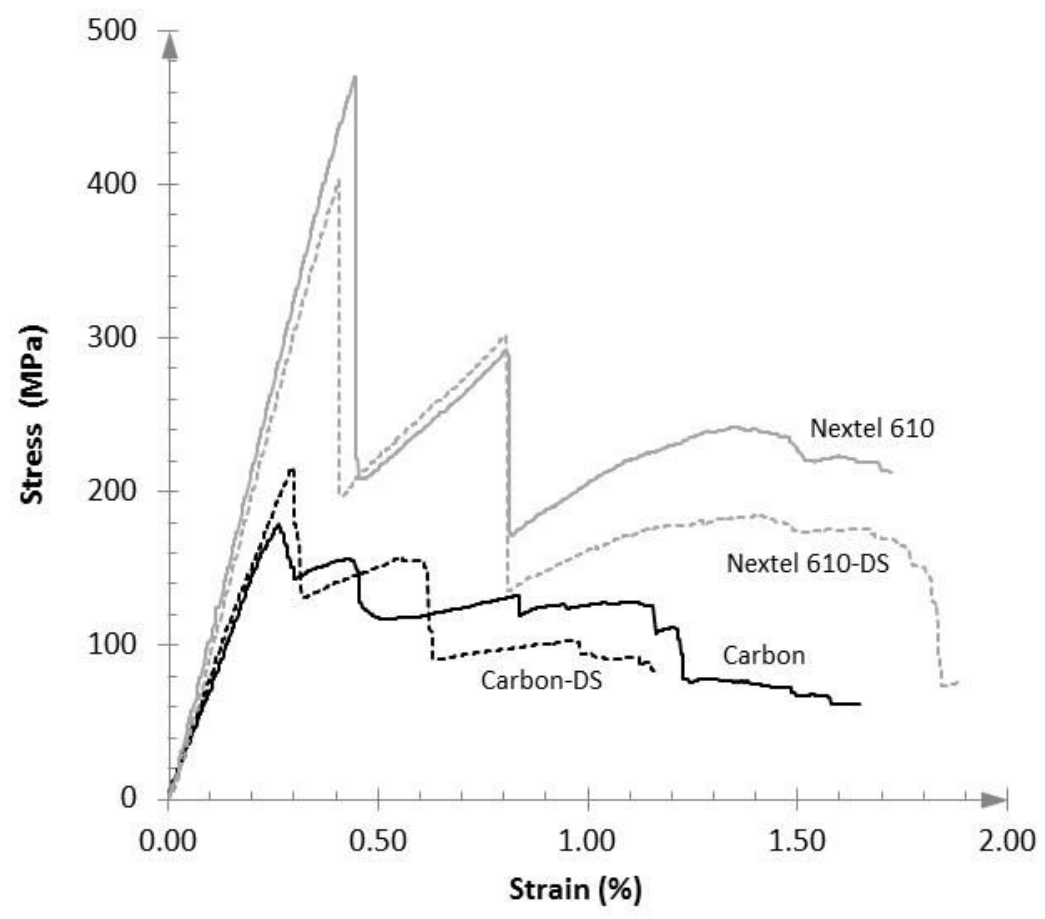

Figure 5.6: Comparison of typical stress-strain behaviour of composites made from sized and desized carbon and Nextel 610 fibres

\subsubsection{Matrix composition and strength}

Compositions M1 and M5 were used to study the influence of the matrix composition and strength on the flexural properties of basalt and carbon fibre composites. In comparison to M1, composition M5 showed an approximately $80 \%$ higher compressive strength. The flexural strength and elastic modulus were about $50-60 \%$ and $20-25 \%$ higher, respectively. The average flexural properties of the different composite types are compared in Table 5.5. M5-Basalt composites achieved a slightly higher average flexural strength of $167 \mathrm{MPa}$ than comparable composites made with M1 matrix (158 MPa). However, the difference is well within the standard deviation measured for each of the two composite series. The range of the strength values is similar to the one observed for M1-Basalt composites and with a minimum and maximum value of 138 and $209 \mathrm{MPa}$ shifted only slightly towards higher strengths. Similarly, the average elastic modulus increased marginally from 31 to 35 GPa for the M5-basalt composites. However, the matrix strength had considerably greater influence on the carbon fibre composites. A clear improvement of both 
average strength and elastic modulus can be observed, as shown in Table 5.5. Except for the weakest sample of the series $(139 \mathrm{MPa})$, the strength of all other M5-Carbon samples exceeded the maximum strength measured for M1-Carbon samples with a maximum strength of $218 \mathrm{MPa}$. However, the higher fibre content of the M5-Carbon composites should be noted. Although it was observed before that a higher fibre content does not necessarily result in higher strength (see Table 5.1), it is most likely to have some effect on the strength. On the other hand, the much higher elastic modulus of the M5-Carbon composites can for the most part be attributed to the higher fibre content.

Table 5.5: Effect of matrix strength on the flexural properties of basalt and carbon fibre composites

\begin{tabular}{lcccc}
\hline Matrix/Fibre & $\begin{array}{c}\mathbf{V}_{\text {fibre }} \\
{[\mathbf{\%}]}\end{array}$ & $\begin{array}{c}\boldsymbol{\sigma}_{\mathbf{f}, \max } \\
{[\mathbf{M P a}]}\end{array}$ & $\begin{array}{c}\mathbf{E}_{\mathbf{f}} \\
{[\mathbf{G P a}]}\end{array}$ & $\begin{array}{c}\boldsymbol{\varepsilon}_{\mathbf{f}} \\
{[\mathbf{\%}]}\end{array}$ \\
\hline M1-Basalt & $29 \pm 3$ & $158 \pm 25$ & $31 \pm 4$ & $0.63 \pm 0.08$ \\
M5-Basalt & $32 \pm 1$ & $167 \pm 29$ & $35 \pm 3$ & $0.56 \pm 0.11$ \\
M1-Carbon & $32 \pm 2$ & $149 \pm 28$ & $59 \pm 7$ & $0.29 \pm 0.09$ \\
M5-Carbon & $40 \pm 1$ & $185 \pm 29$ & $94 \pm 8$ & $0.23 \pm 0.02$ \\
\hline
\end{tabular}

Typical stress-strain curves of basalt and carbon fibre composites with M1 and M5 matrix are displayed in Figure 5.7. M5-Basalt composites generally show a nearlinear elastic behaviour up to the point of maximum stress similar to M1-Basalt composites. Beyond this point, however, the M5-Basalt composites show a more brittle behaviour than their M1 counterparts with an essentially instantaneous decrease of the stress level to roughly $20-70 \mathrm{MPa}$. Upon further loading the stress continues to slowly decrease. The differences between the behaviour of the two carbon fibre composites, however, are less obvious and the behaviour of the stressstrain curves of the M5 composites is less consistent. Overall, the initial stress-strain behaviour of the two carbon fibre composites appears to be largely similar. However, the stress level seems to decrease more rapidly for the stronger M5-composites after the initial failure occured. In terms of the appearance of the macroscopic failure patterns, no significant difference between the respective composites with the two different matrices was observed. 


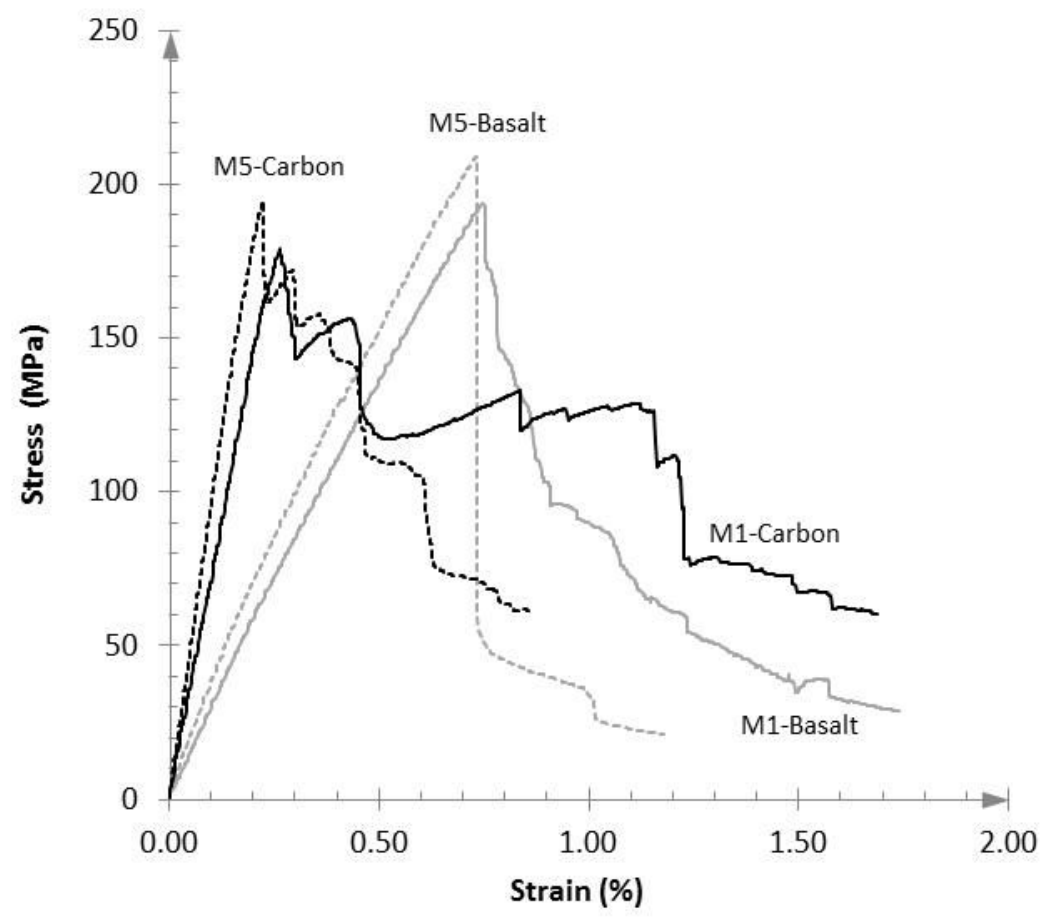

Figure 5.7: Effect of matrix strength on the stress-strain behaviour of basalt and carbon fibre composites

\subsubsection{Drying time}

The effect of drying time was studied exemplarily on M1-Basalt fibre composites after seven and 28 days. Table 5.6 shows an increase of the average flexural strength from 158 to $181 \mathrm{MPa}$ with increasing drying time. The strength values of the 28 day sample series ranged between a minimum and maximum of 132 and $224 \mathrm{MPa}$. Whereas the maximum strength was notably higher than for the seven day sample series (194 MPa), the minimum value was approximately similar for both composite series. The strength values of the remaining samples were in the same range as the seven day sample series. Therefore, it is not clear how significant the effect of the drying time really is. It should be noted that due to a data recording error, the 28 day sample series only comprised of four samples. The average elastic modulus was slightly higher for the 28 day sample series. 
Table 5.6: Effect of drying time on the flexural properties of basalt fibre composites

\begin{tabular}{lcccc}
\hline Drying time & $\begin{array}{c}\mathbf{V}_{\text {fibre }} \\
{[\mathbf{\%}]}\end{array}$ & $\begin{array}{c}\boldsymbol{\sigma}_{\mathbf{f}, \max } \\
{[\mathbf{M P a}]}\end{array}$ & $\begin{array}{c}\mathbf{E}_{\mathbf{f}} \\
{[\mathbf{G P a}]}\end{array}$ & $\begin{array}{c}\boldsymbol{\varepsilon}_{\mathbf{f}} \\
{[\mathbf{\%}]}\end{array}$ \\
\hline Basalt - 7 days & $29 \pm 3$ & $158 \pm 25$ & $31 \pm 4$ & $0.63 \pm 0.08$ \\
Basalt - 28 days & $32 \pm 6$ & $181 \pm 38$ & $34 \pm 3$ & $0.64 \pm 0.18$ \\
\hline
\end{tabular}

The failure behaviour, as displayed by the stress-strain curves in Figure 5.8, remained largely unaffected over time. Although some variation between the general stress-strain behaviour of the 28 day samples can be observed, a similar variation is evident for the seven day samples. Also, no change of the macroscopic failure pattern over time was observed.

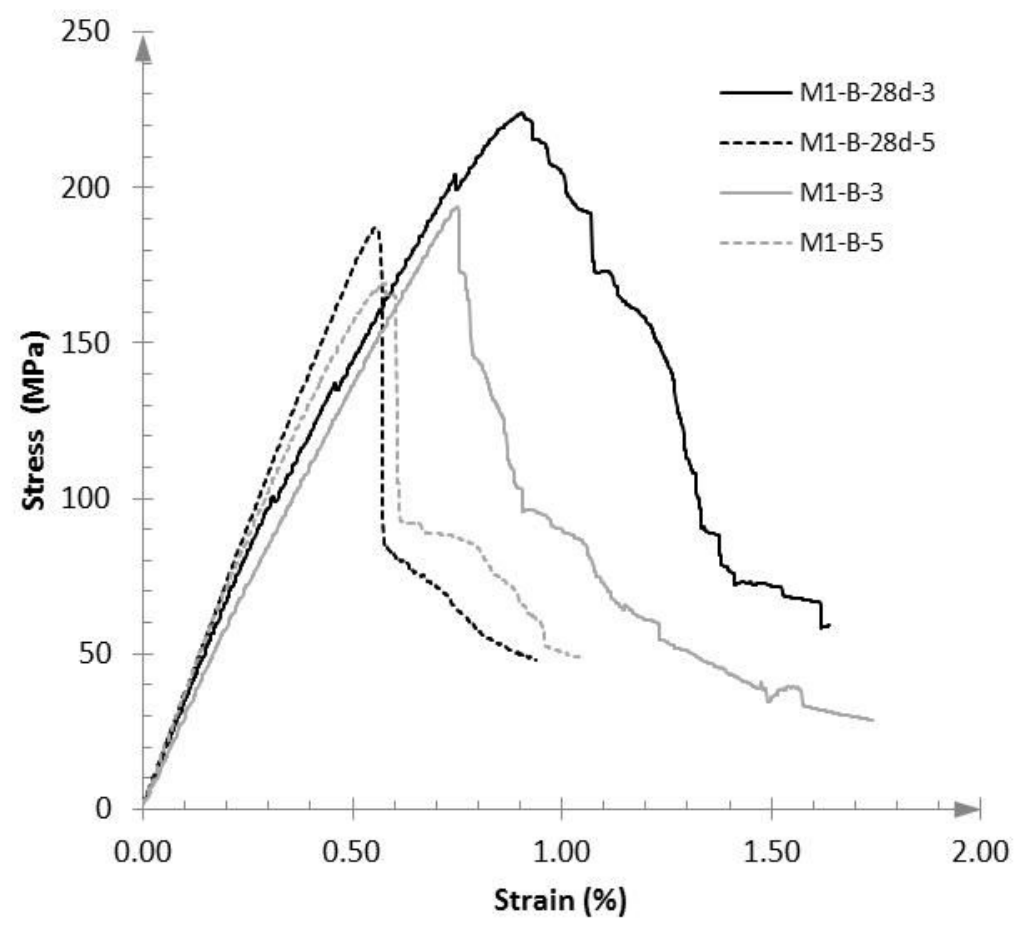

Figure 5.8: Comparison of stress-strain curves of basalt fibre composites after seven and 28 days drying time

\subsubsection{Specimen dimensions and span-to-depth ratio}

The influence of the span-to-depth ratio and the composite bar thickness was investigated exemplarily on basalt fibre composites. As mentioned before, the 
standard bar thickness for all composite samples was aimed at around three millimetres with a fix testing span of $100 \mathrm{~mm}$. Although the actual s/d ratio varied to some extent due to the variability of the bar thickness of individual samples, an s/d ratio of roughly 32:1 shall be assumed for all samples tested under these conditions. These results were compared to the results achieved for an $\mathrm{s} / \mathrm{d}$ ratio of approximately 16:1, which is the minimum recommended span-to-depth ratio for three-point flexural strength testing of composites by several testing standards, e.g. ASTM D790 and ASTM C-1341. The following two variations were investigated: i) a reduced support span of $50 \mathrm{~mm}$ while keeping the sample thickness unchanged, and ii) a sample thickness of approximately six millimetres while keeping the support span fix at $100 \mathrm{~mm}$. The samples for the testing on the $50 \mathrm{~mm}$ span were obtained by simply cutting three standard sized bars in half. The six millimetre bars were prepared by the lay-up of two times 10 impregnated basalt fibre tapes in the same mould. The fabrication of these bars was very difficult. Therefore, only two of these samples were tested. Both bars were prepared form the same batch of geopolymer binder.

Table 5.7: Effect of specimen thickness and span-to-depth ratio on the flexural properties of basalt fibre composites

\begin{tabular}{lllcc}
\hline s/d ratio / span & $\begin{array}{c}\mathbf{V}_{\text {fibre }} \\
{[\%]}\end{array}$ & $\begin{array}{c}\boldsymbol{\sigma}_{\mathbf{f}, \max } \\
{[\mathbf{M P a}]}\end{array}$ & $\begin{array}{c}\mathbf{E}_{\mathbf{f}} \\
{[\mathbf{G P a}]}\end{array}$ & $\begin{array}{c}\boldsymbol{\varepsilon}_{\mathbf{f}} \\
{[\mathbf{\%}]}\end{array}$ \\
\hline $32: 1 / 100 \mathrm{~mm}$ & $29 \pm 3$ & $158 \pm 25$ & $31 \pm 4$ & $0.63 \pm 0.08$ \\
$16: 1 / 50 \mathrm{~mm}$ & $32 \pm 2$ & $126 \pm 19$ & $27 \pm 2$ & $0.51 \pm 0.07$ \\
$16: 1 / 100 \mathrm{~mm}$ & $32 \pm 1$ & $111 \pm 3$ & $29 \pm 2$ & $0.38 \pm 0.02$ \\
\hline
\end{tabular}

Table 5.7 and Figure 5.9 both show that the s/d ratio and the bar thickness have significant influence on the measured strength and the failure behaviour. A considerably lower strength was measured for the two sample series with the $16: 1 \mathrm{~s} / \mathrm{d}$ ratio compared to the standard type samples. The difference between these two testing configurations lies within the standard deviation of the $50 \mathrm{~mm}$ span samples which achieved strength values between 91 and $143 \mathrm{MPa}$. However, it appears that for the same s/d ratio a somewhat lower strength can be expected with increasing sample thickness. The very low deviation between the strength of the two six millimetre samples, however, is somewhat surprising. The difference between the 
average elastic moduli is less significant. Nevertheless, slightly higher elastic moduli seem to be achieved over a larger support span.

The failure behaviour changed significantly between the two different $\mathrm{s} / \mathrm{d}$ ratios as shown in Figure 5.9. On the other hand, no significant differences in failure behaviour were observed between the two testing configurations with the same s/d ratio. In comparison to the behaviour of the standard composites with a $32: 1 \mathrm{~s} / \mathrm{d}$ ratio as described before, the stress-strain curves of the two 16:1 samples show a step-like behaviour which is similar to the one observed for the standard carbon composites. The macroscopic failure patterns of the 16:1 samples are also similar are dominated by interlaminar shear failure and various extents of fibre buckling (Figure 5.10). Only the six millimetre thick samples showed a small amount of fibre fracture on the tensile side.

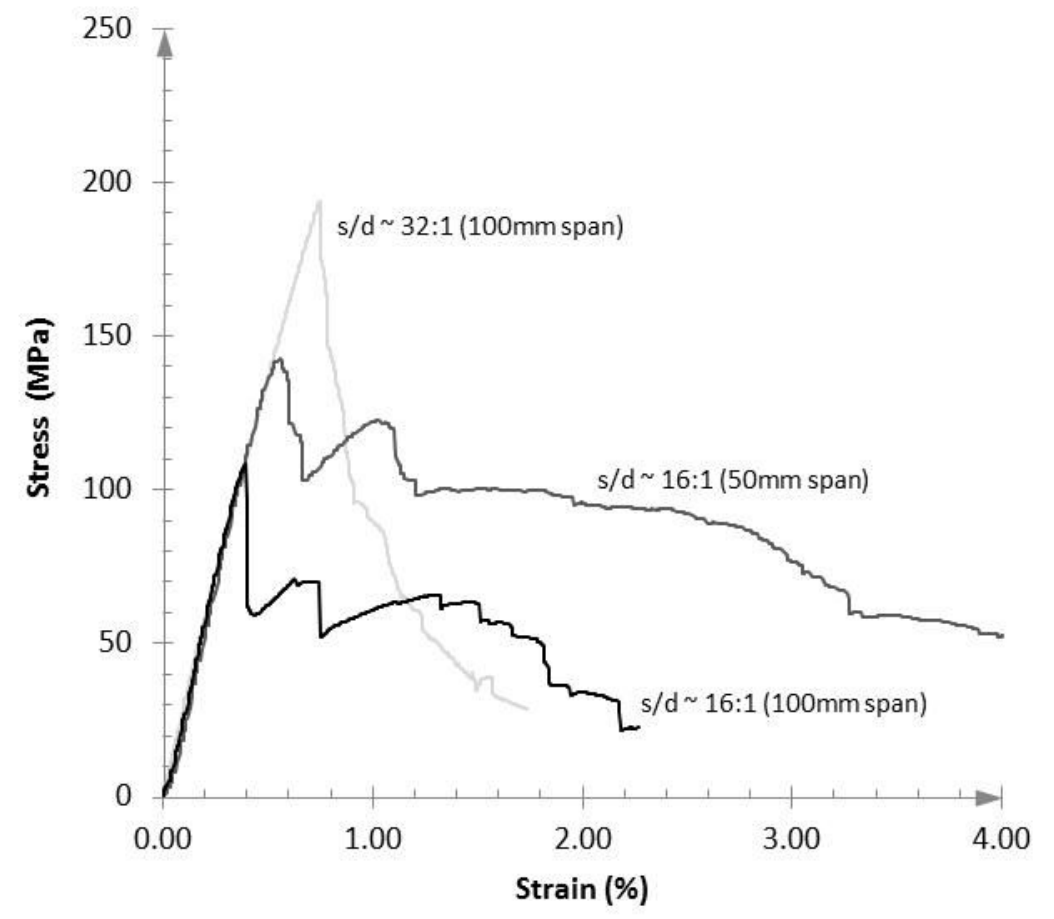

Figure 5.9: Effect of sample thickness and span-to-depth ratio on the stress-strain behaviour of basalt fibre composites 


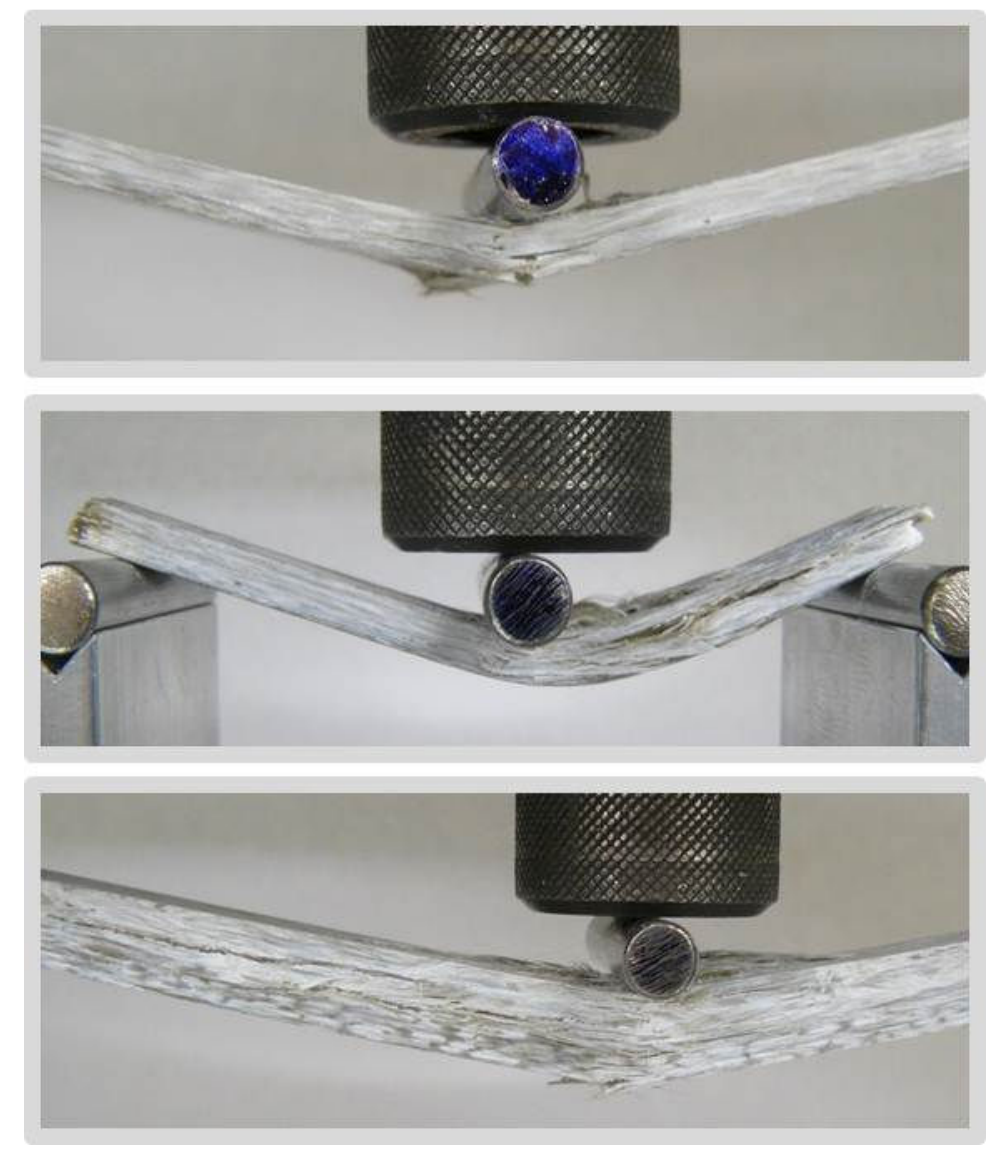

Figure 5.10: Typical macroscopic failure observed for UD basalt fibre composites depending on sample thickness and span-to-depth ratio: s/d $\sim 32: 1,100 \mathrm{~mm}$ span (top), s/d $\sim 16: 1,50 \mathrm{~mm}$ span (middle) and $\mathrm{s} / \mathrm{d} \sim 16: 1,100 \mathrm{~mm}$ span (bottom).

\subsection{In-plane flexural strength}

The in-plane strength was determined for basalt and carbon fibre composites. Suitable samples for the in-plane strength tests were obtained by cutting standard composite bars across their length into half. The cut surface of each bar was subsequently ground to a width of $\sim 3 \mathrm{~mm}$ to obtain a roughly square cross-section. One of the two $3 \times 3$ bars cut from of each standard composite bar was tested in inplane and the other one in out-of-plane/through-thickness orientation. This allowed a direct comparison between the two results.

The results of the in-plane and out-of-plane flexural strength tests in comparison to the results achieved in the standard testing of the same types of composites are 
presented in Table 5.8 and Figure 5.11. The average strength of the basalt fibre composites in in-plane mode was found to be only slightly higher compared to the out-of-plane orientation with values of 186 and $169 \mathrm{MPa}$, respectively. However, the standard deviation of the in-plane samples was significantly higher. The first two samples achieved similar strength in both testing configurations with a minimum strength of around $130 \mathrm{MPa}$. Sample three achieved the highest strength in both tests with 198 and $240 \mathrm{MPa}$ for the out-of-plane and in-plane orientation, respectively. An average elastic modulus of $\sim 40 \mathrm{GPa}$ was determined for the $3 \times 3$ samples in both tests. Compared to the standard test configuration, the smaller bars achieved slightly higher average strength and a higher elastic modulus. However, the difference between the two sample types is most likely a result of the higher fibre content of the smaller 3 x 3 basalt samples.

Table 5.8: Comparison of in-plane and out-of-plane flexural properties of basalt and carbon fibre composites

\begin{tabular}{|c|c|c|c|c|}
\hline & $\begin{array}{l}\mathbf{V}_{\text {fibre }} \\
{[\%]}\end{array}$ & $\begin{array}{c}\sigma_{\mathbf{f}, \max } \\
{[\mathrm{MPa}]}\end{array}$ & $\begin{array}{c}\mathbf{E}_{\mathbf{f}} \\
{[\mathbf{G P a}]}\end{array}$ & $\begin{array}{c}\varepsilon_{\mathrm{f}} \\
{[\%]}\end{array}$ \\
\hline Basalt standard & $29 \pm 3$ & $158 \pm 25$ & $31 \pm 4$ & $0.63 \pm 0.08$ \\
\hline $3 \times 3$ out-of-plane & $37 \pm 3$ & $169 \pm 25$ & $39 \pm 3$ & $0.53 \pm 0.07$ \\
\hline $3 \times 3$ in-plane & $37 \pm 3$ & $186 \pm 40$ & $41 \pm 4$ & $0.62 \pm 0.13$ \\
\hline Carbon standard & $32 \pm 2$ & $149 \pm 28$ & $59 \pm 7$ & $0.29 \pm 0.09$ \\
\hline $3 \times 3$ out-of-plane* & $38 \pm 5$ & $193 \pm 53$ & $79 \pm 14$ & $0.48 \pm 0.15$ \\
\hline $3 \times 3$ in-plane* & $38 \pm 5$ & $318 \pm 51$ & $83 \pm 9$ & $0.47 \pm 0.06$ \\
\hline
\end{tabular}

The difference between the in-plane and out-of-plane strength of the $3 \times 3$ samples as well as the standard samples was much more significant for the carbon fibre composites. The in-plane samples generally achieved a more than $100 \mathrm{MPa}$ higher strength than their respective equivalents tested in out-of-plane configuration with a maximum strength of $359 \mathrm{MPa}$. The average elastic modulus in both cases was similar at $\sim 80 \mathrm{GPa}$. Both strength and modulus of the $3 \times 3$ out-of-plane samples were considerably higher than the respective values achieved in the standard test. To some degree, this may be attributed to the higher fibre content of the $3 \times 3$ samples. 

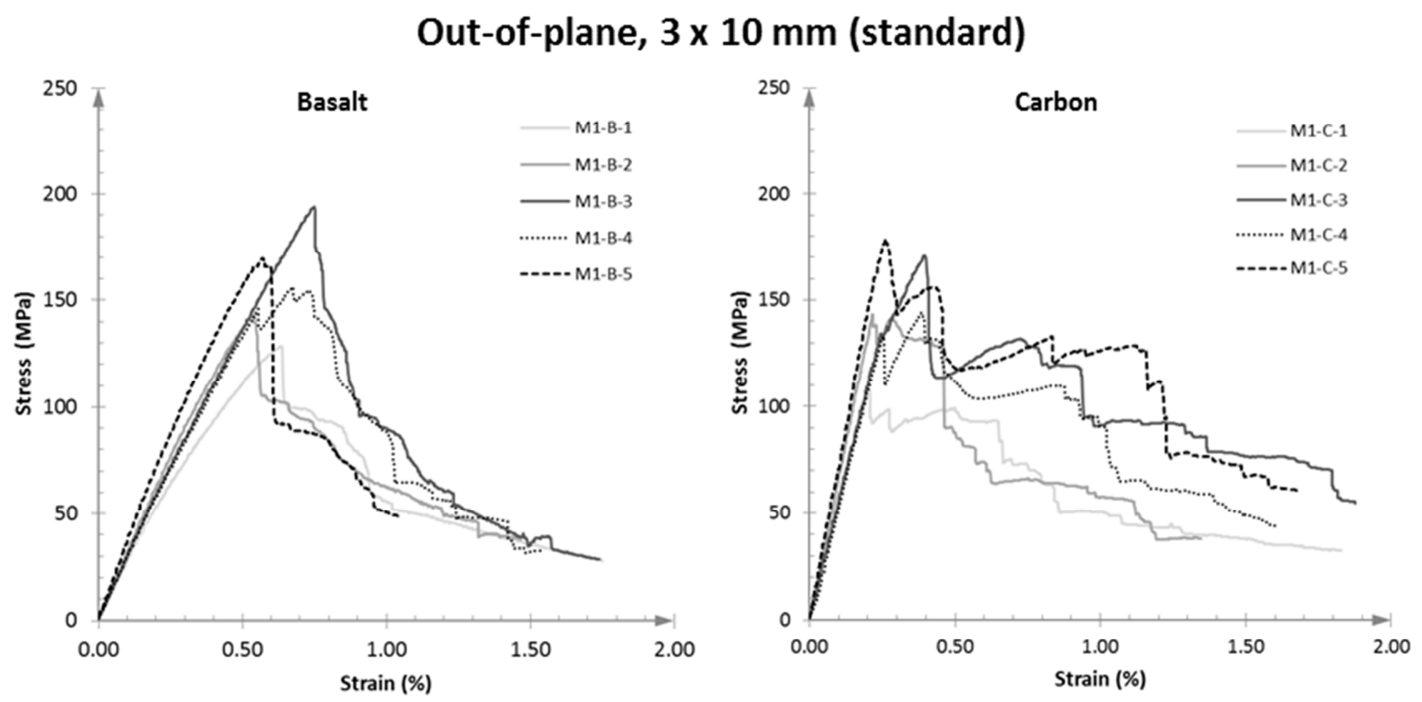

Out-of-plane, $3 \times 3 \mathrm{~mm}$
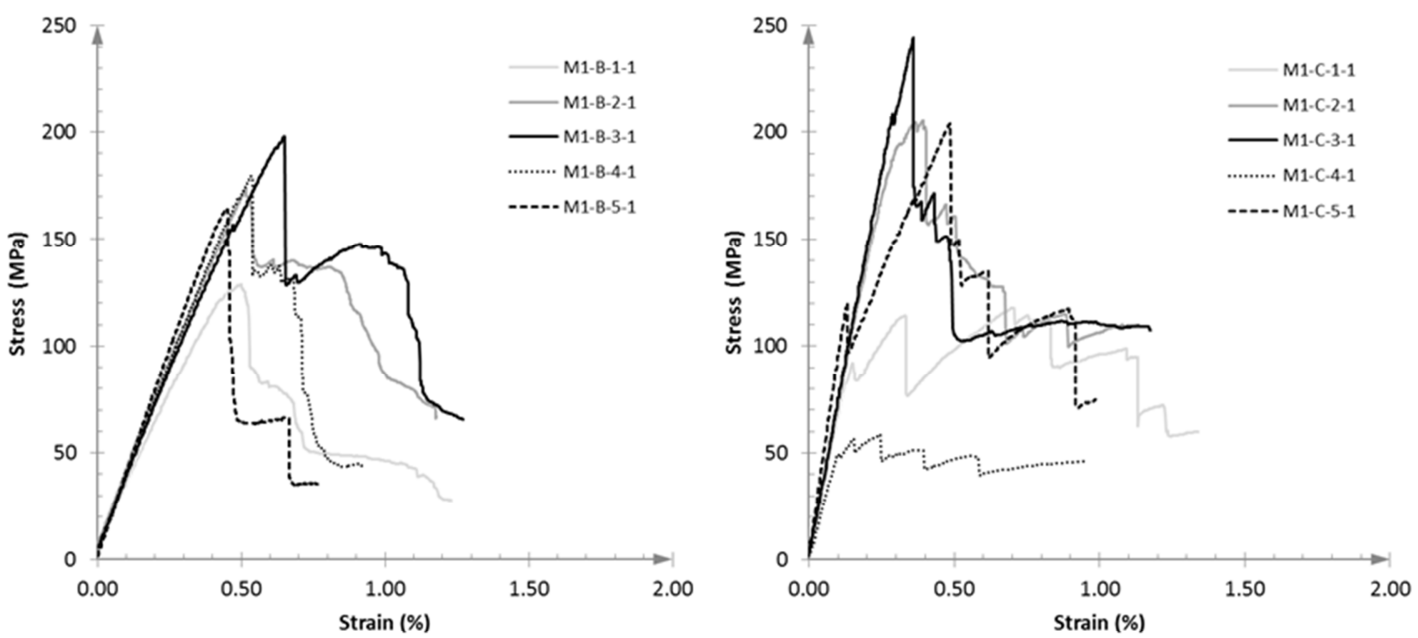

In-plane, $3 \times 3 \mathrm{~mm}$
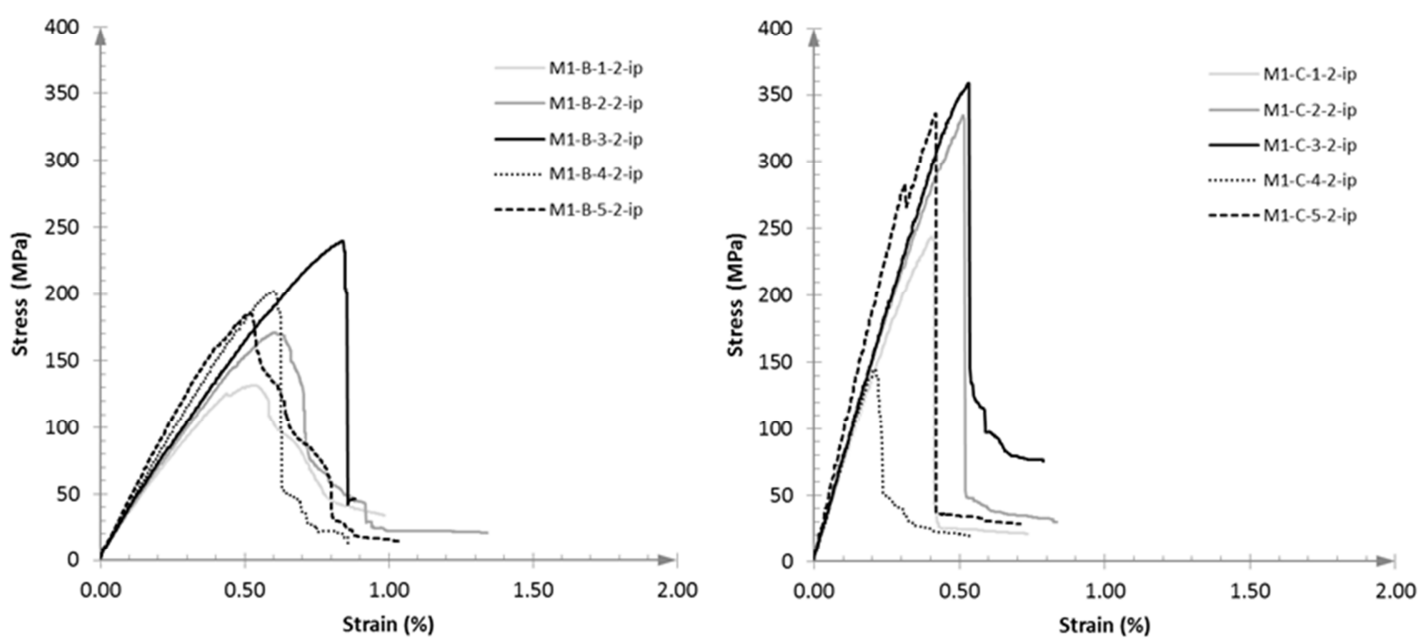

Figure 5.11: Stress-strain curves recorded for in-plane and out-of-plane flexural testing of basalt (left) and carbon (right) composites 
The recorded stress-strain curves are shown in Figure 5.11. In particular the $3 \times 3$ out-of-plane basalt fibre composites showed some variation in the general behaviour of the stress-strain curves. A more pronounced flattening of the stress-strain curves near the point of maximum stress was observed for most of the in-plane basalt samples. The $3 \times 3$ out-of plane samples generally showed similar failure behaviour to the standard composite bars. The carbon composites characteristically failed by delamination (shear) and fibre buckling. The basalt composites in most cases showed some amount of fibre fracture on the tensile side with various degrees of fibre buckling and small delamination cracks. Photographs of typical failure observations for the in-plane basalt and carbon specimens are shown in Figure 5.12. A small amount of fibre fracture was observed for all basalt specimens. In some cases the formation of cracks between the different fibre layers, as shown in Figure 5.12b), could also be noticed. Twisting of the bars in the testing jig was observed in several instances for both basalt and carbon specimens and is exemplarily shown in Figure 5.12d) and e). Despite the sample orientation, delamination between the fibre layers in the original lay-up direction around the point of central loading as well as significant matrix fragmentation was observed for the carbon specimens. Although
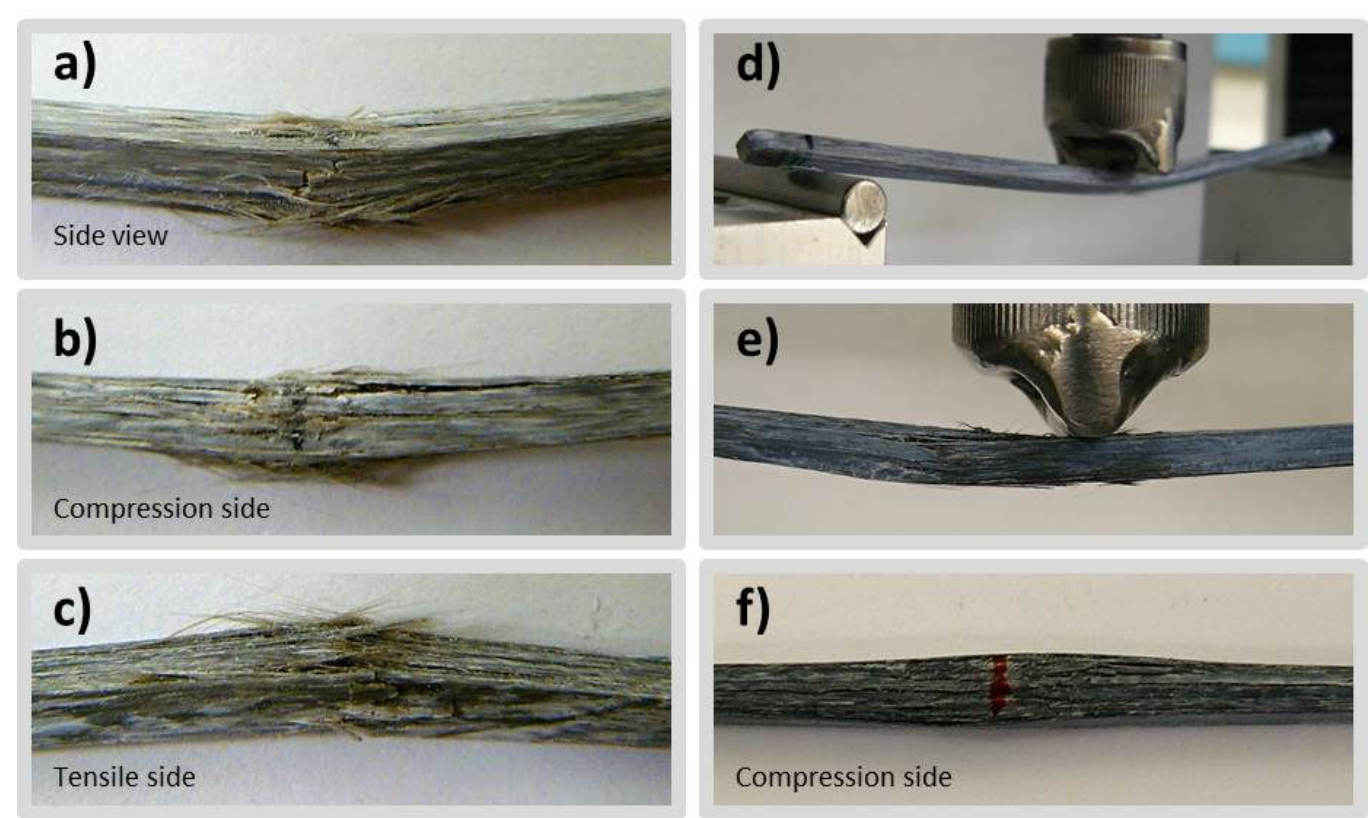

Figure 5.12: Typical in-plane failure patterns observed for basalt $(a-c)$ and carbon $(d-f)$ fibre composites 
the delamination cracks are hardly evident from the photograph in Figure 5.12f), the slight widening of the sample width around the centre point indicates some amount of delamination in that area. In combination with the torsion of the sample, this appeared to be the main failure mode for the carbon specimens in in-plane testing.

\subsection{Short beam shear tests}

Short beam tests were carried out to determine the interlaminar shear strength of basalt and carbon fibre composites. For the purpose of this test, standard composite bars were cut into five pieces of $\sim 23 \mathrm{~mm}$ length using a diamond saw and IPA as a coolant. Two standard composite bars, more precisely the first and third bar of a fabrication series, were compared for both fibre types. For better clarity, these samples are subsequently simply referred to as composite bars $\mathrm{CB} 1$ and $\mathrm{CB} 3$. Several support spans were tested in preliminary experiments. The final tests were carried out on a $10 \mathrm{~mm}$ span with a respective span-to-depth ratio of $\sim 4: 1$.

The basalt fibre composites typically failed in compression and tensile failure for testing spans between 10 and $20 \mathrm{~mm}$. However, shear failure, i.e. delamination, is an essential requirement for the validity of the test result. Therefore, no interlaminar shear strength could be calculated for basalt fibre composites. On the other hand, delamination was observed as the main failure mechanism for carbon fibre composites. Average interlaminar shear strengths of $7.8 \pm 0.4 \mathrm{MPa}$ and $9.2 \pm 0.3$ $\mathrm{MPa}$ were calculated for composite bars $\mathrm{CB} 1$ and $\mathrm{CB} 3$ on a $10 \mathrm{~mm}$ span, respectively. Lower strengths were measured for larger testing spans. Interlaminar shear strengths between 5.5 - 6 MPa were measured for a CB1 composite bar on a 15 $\mathrm{mm}$ span. However, an increased bending of the short beam samples on the $15 \mathrm{~mm}$ compared to the $10 \mathrm{~mm}$ span was noticeable. 


\subsection{Discussion}

As suggested by the literature, this study clearly confirmed that the incorporation of strong and stiff inorganic fibres into a geopolymer matrix not only prevents catastrophic failure but also leads to a significant improvement of essentially all mechanical properties. However, the mechanical properties are influenced by a number of parameters as shown in the previous chapters. While some of these results are consistent with previous studies others are contradictory. In the following section, the various parameters that influence the mechanical properties of geopolymer matrix composites and their general failure behaviour are critically discussed in comparison to the current literature (see chapter 2.3).

\subsubsection{The influence of fabrication process and processing variables}

In chapter 4 , the general behaviour and performance of the geopolymer matrix was investigated and discussed in detail. Many of the aspects that were discussed in the context of the unreinforced geopolymer matrix also have to be considered for respective composites. One of the main problems throughout that part of the study was the reproducibility of the mechanical properties between different batches of geopolymer binder. It is unclear how this problem translates to the composite specimens. This leaves some uncertainty over the actual strength of the matrix in the various composite specimens. It is arguable if the properties of the strongest batch of composition M1 and M5, which were assumed in the previous chapter to present a more accurate representation of the true properties of the respective composition, can also be assumed as the actual matrix properties of the composite specimens. However, for reasons of simplicity the following assumptions are made for the purpose of this discussion: (i) the matrix properties are relatively consistent and show only little variation between different batches of geopolymer binder and (ii) irrespective of the actual strength values, composition M5 is stronger than M1.

In the beginning of chapter 5.1, the significant influence of the fabrication process on the flexural strength of the composites was discussed. Closer analysis of the flexural strength data revealed a dependency of the strength on the order of fabrication within 
each set of composite specimens, irrespective of the fibre type or any other parameter. As mentioned before, three composite samples could be prepared from each batch of geopolymer binder. In almost all cases a gradual increase in flexural strength could be observed from the first to the third sample of a fabrication series. In the following paragraph, possible explanations for this behaviour are explored.

Apart from the inherent variability that inevitably results from an all-manual fabrication process, only two distinguishable variables can be identified in the fabrication process of the three samples of any given set of composite specimens. The first one is the mixing time of the geopolymer binder and the second one is related to a difference in the early setting/curing regime of the three samples. As described before, a continuous mixing process was used in order to increase the effective use-time of the geopolymer binder, thereby increasing the number of composite specimens that could be produced from one batch of geopolymer binder. Thus, one of the differences between the three composite specimens was the time the matrix binder was mixed for. The investigation of the unreinforced geopolymer showed some indication of increasing compressive strength for extended mixing times. Therefore, it may be possible that the increasing flexural strength of the composite specimens is somehow related to an increase of the compressive strength of the matrix. Furthermore, the competition between the onset of the geopolymerisation process and the retardation of the setting of the geopolymer binder due to the constant application of shear stresses during the continuous mixing process was discussed. As a result, the effective use-time of the geopolymer binder was progressively shortened with increasing mixing time. This may affect the network formation of the geopolymer matrix itself as well as the strength of the fibre matrix bond. However, the microstructural analysis of the composites, which is addressed in more detail in chapter 6 , revealed no notable microstructural difference between the three specimens of a fabrication series. The faster setting of the geopolymer binder after longer mixing times might also positively affect the interlaminar bonding between the different fibre layers in each composite. The conjecture of increasing interlaminar shear strength in the order of fabrication of the three samples within each fabrication series is supported by the results of the short 
beam shear tests of carbon fibre composites. However, further experiments on this aspect are required to determine what exactly causes the apparent increase of the interlaminar shear strength of the matrix.

The second variable is also a practical consequence of the particular fabrication process and the continuous mixing method used in this study. In order to save valuable time during the fabrication process, the first and second sample were not immediately placed in the oven at $40^{\circ} \mathrm{C}$ but were stored in a sealed plastic bag on the lab bench until the third samples was fabricated before placing all three sample in the oven for curing. This means that the initial setting and curing stage of the three samples was slightly different. By the time the third sample had been fabricated, the first sample had already been curing at room temperature for approximately 45 minutes. While it is known that the two main curing parameters, i.e. time and temperature, can have significant effect on the mechanical properties of geopolymers, the role of this initial curing stage is less clear. However, a comparative study of the reaction kinetics of $\mathrm{NaOH}$-activated metakaolin at temperatures of 25 and $40^{\circ} \mathrm{C}$ by Zhang et al. found that the increase of the reaction temperature increases the initial reaction rate but has little effect on the final reaction extent [Zhan12]. Nevertheless, it may be possible that the different initial reaction rate affects other parameters such as shrinkage rate or porosity distribution in the matrix which could influence the fibre-matrix interaction.

While the possible effects associated with the longer mixing time appear to be more dominant than the ones related to the different initial curing and reaction rate, a potential interaction between the different effects is likely. In any case, it is clear that the strength dependency of the composites on the order of their fabrication is the result of a change in the properties of the geopolymer matrix and a direct consequence of the particular fabrication process chosen for this study. Due to these unintended side effects, the continuous mixing concept that was used here has to be considered unfavourable for the production of geopolymer composites. While other fabrication methods may appear more suitable, ensuring the consistency of all fabrication parameters, in particular the ones related to the geopolymer matrix, seems 
to be a critical factor for achieving good reproducibility of results. On the other hand, the current results help to illustrate the possible influences of varying processing parameters on the mechanical behaviour of geopolymer matrix composites. These insights are invaluable when considering potential scale-ups processes.

\subsubsection{The effect of fibre type on the mechanical properties}

The results showed that the mechanical properties of the composites are influenced by a number of parameters. Of all parameters that were investigated in this study, the fibre type has the strongest impact on the performance of the composite. Because the strength and stiffness of all investigated fibre types are significantly higher compared to the geopolymer matrix, the mechanical properties of all composites are strongly fibre dominated. However, considerable differences between the different fibre types can be observed. The influence of the fibre type on the flexural properties of the composites was shown in Table 5.2. Of the three main fibre types that were investigated, alumina fibre composites showed by far the highest strength and stiffness. Both the basalt and carbon fibre composites achieved roughly similar strength. However, the elastic modulus of the carbon composites was nearly double the value of the basalt composites. This is due to the much higher elastic modulus of the carbon compared to the basalt fibre (see Table 3.2). Despite the fact that the elastic moduli of the three composite types generally correlate with the elastic moduli of the fibres, the moduli of the carbon as well as the alumina composites are lower than expected. The expected elastic moduli of the composites were estimated from the volume content and the elastic moduli of the respective fibre type and the M1 matrix according to the rule of mixture. Whereas the average elastic modulus of the basalt composites was in good agreement with the estimated value ( 31 vs. 33 $\mathrm{GPa}$ ), the measured moduli of both the carbon and alumina fibre composites lag behind their estimated values (carbon: 59 vs. $84 \mathrm{GPa}$; alumina: 105 vs. $122 \mathrm{GPa}$ ). The reasons for this discrepancy are not fully understood. To some degree this may be attributed to the somewhat inaccurate deflection measurement. However, the good correlation of the two values for the basalt composites suggests that the error that results from the deflection measurement is only small. Microstructural defects due to imperfect impregnation of the fibre tapes and the general wettability of the fibres 
may also be considered as possible factors affecting the elastic modulus of the composite. Although the particular aspect of the discrepancy between the measured and the estimated elastic modulus was not investigated in any more detail, the reader is referred to chapter 6 for more information on the microstructural interaction between fibre and matrix.

Unlike the correlation between the elastic moduli of the composites and fibres, the flexural strength of the composites stands in no relation to the fibre strength. In fact, the strength of the composites appears to be controlled much more by the stiffness and strain capacity of the fibre than by the fibre strength. This is evident from the comparison of the results for the basalt and alumina composites. Whereas the alumina fibres are nominally the weakest but stiffest fibre of the three main fibre types used here, the respective composites achieved the highest flexural strength. Vice versa, the basalt fibres are the strongest but least stiff fibres in the test. The respective composites, however, showed significantly lower strength compared to the alumina composites. Despite the highest fibre strength, the basalt composites were also the only composite type that showed an appreciable amount of fibre fracture due to tensile failure. This suggests that the fibre strength is not a major factor for achieving high-strength geopolymer matrix composites. On the other hand, the use of a high modulus fibre does not necessarily result in a high-strength composite as is evident from the results of the carbon fibre composites. Given the much higher modulus and only slightly lower strength of the carbon compared to the basalt fibres, much higher strengths would be expected for the carbon composites. However, both composite types achieved roughly similar strength. Two aspects may be considered in order to explain the strength of the carbon composites. In anticipation of the microstructural analysis of the fibre-matrix interaction in chapter 6 it can already be revealed that the wettability of the carbon fibres by the matrix was very poor. The poor wettability of the carbon fibre and the weak fibre-matrix bond are believed to be at least partly responsible for the seemingly low strength of the carbon composites. The second factor is related to the calculation of the flexural strength itself. This aspect was already briefly addressed in chapter 2.3 and is not just relevant to the present work but large parts of the literature [e.g. 9, 11, 13]. In accordance with 
previous studies, standard beam theory was applied to calculate the flexural strength of the rectangular composite samples. If this theory and in particular its assumption of uniform compressive and tensile strains during flexural loading applies to geopolymer matrix composites (or more generally composites combining very strong and stiff fibres with very weak matrices) in the first place is arguable and has to be investigated in more detail in future studies. However, another more obvious criterion that is required for the valid calculation of a flexural strength is tensile failure. Since most composites, with the exception of the basalt fibre composites, failed predominantly in shear mode, the calculation of a flexural strength from this loading configuration is in fact invalid. This aspect has been largely neglected so far. As a result, the reported strengths of the carbon and alumina composites, as well as all other composite types that failed in shear, may rather be seen as the apparent flexural stresses at which (shear) failure occurs. However, these values do not represent the actual flexural strength of these composites. The ultimate flexural strength of these composites should therefore be higher. The same is true for respective studies in the literature where shear was observed as the main failure mode. Whereas, for the purpose of this discussion, the calculated maximum apparent stress is continued to be referred to as the flexural strength for reasons of consistency within this study and for comparison with the literature, the above considerations have to be kept in mind. The general stress-strain and failure behaviour of geopolymer matrix composites and the role of the fibre properties therein are discussed in more detail in a subsequent section of this discussion.

The combination of basalt with carbon and boron fibres, respectively, resulted in the envisaged increase of the elastic modulus compared to the basalt composites. This observation is particularly interesting from an economical point of view as it offers a relatively cost-efficient way to significantly increase the composite stiffness while still using the much cheaper basalt fibres as the main reinforcement type. But whereas the addition of boron fibres also resulted in an increased composite strength, a decreased strength was observed for the basalt-carbon composites compared to the purely basalt fibre reinforced composites. It appears somewhat curious that the combination of basalt and carbon fibres results in a lower strength than the 
composites with either of the two fibre types taken separately. The reasons for this behaviour can only be speculated. A possible explanation may be found in the different flexibility of the two distinct fibre layers in combination with the bad wettability and weak fibre-matrix bond of the carbon fibres, respectively. Upon loading, the higher stiffness of the carbon layers inhibits the deformation of the basalt layers causing additional stresses between the two different fibre layers. Due to the weak bonding between the matrix and the carbon fibres, these stresses may result in the local detachment of the two fibre layers which leads to the premature failure of the basalt-carbon composites.

In comparison to the existing literature, the superior performance of the alumina over the carbon composites at similar fibre content principally confirms previous observations by Foerster for composites made with aluminosilicate and carbon fabrics, respectively [3]. The basalt fibre composites, on the other hand, offer an interesting alternative to the predominantly investigated carbon composites in terms of flexural strength and particularly from an economical point of view. They also show significant advantages compared to previously investigated glass fibre composites [11] such as higher strength and non-catastrophic failure behaviour. Since carbon fibres are the most widely investigated reinforcement type for geopolymer composites this composite type offers the largest basis for comparison. In terms of the overall flexural strength, the current results are significantly lower than the highest values reported for other unidirectional carbon fibre composites $[3$, $9,11]$. However, the comparability between these studies is limited due to varying fabrication methods, fibre content, matrix composition, testing parameters, etc. Also, achieving maximum composite strength was not the objective of this investigation. The main goal was rather to investigate how different parameters affect the properties of different composite types relative to each other. But despite the fact that significant strength improvements are undoubtedly feasible with more advanced fabrication methods and higher fibre contents, flexural strengths of a carbon fibre geopolymer matrix composite of $>500 \mathrm{MPa}$ in an out-of-plane bend test seem extremely high. On the other hand, the strength values reported by He et al. [13] are in much better agreement with the present results. These authors report a flexural 
strength and elastic modulus of $133 \mathrm{MPa}$ and $37 \mathrm{GPa}$, respectively, for a unidirectional carbon fibre reinforced composite with a fibre volume content of $20-$ $25 \%$. Given the $5-10 \%$ lower fibre content, these values are close to the flexural strength and modulus measured in the present work. Further similarities between the two studies are found in regards to the general stress-strain behaviour of the composites under loading and the observation of predominantly shear failure. On the other hand, despite the fact that Foden [9] and Hammell [11] describe similar failure criteria as observed in the present study, i.e. fibre buckling and shear failure, the general behaviour of the stress-strain curves reported by these authors is inherently different to the behaviour observed here.

\subsubsection{Other factors influencing the mechanical properties}

Besides the major influence of the fibre type, other parameters were also found to affect the mechanical properties of geopolymer matrix composites. In the following section, the influence of fibre sizing, matrix strength and testing parameters are critically discussed.

The results of the investigation of the influence of the fibre sizing on carbon and alumina composites were displayed in Table 5.4 and Figure 5.6. Based on these results, a general effect of the fibre sizing on the composite strength cannot be clearly identified. The desizing appears to have a positive effect on the strength and elastic modulus of the carbon fibre composites. However, the higher fibre content and the large deviation of the strength of the desized composites make it difficult to clearly identify this effect. In case of the alumina composites, the effect of the fibre sizing on the composite strength is even smaller. If at all, slightly higher composite strength may be achieved with sized rather than desized fibres. It is concluded that the fibre sizing is likely to have some effect on the strength and stiffness of geopolymer matrix composite. However, the impact of this parameter appears to be relatively small compared to the influence of the fibre type. A general trend of the influence of the fibre sizing on the mechanical properties cannot be observed since the effect of the fibre sizing appears to depend on the fibre type and the particular nature of the fibre sizing. In that regard, the epoxy sizing of the carbon fibre seems less favourable 
than the sizing of the alumina fibres. Irrespective of any possible effect of the fibre sizing on the mechanical properties, it can be established that the fibre sizing or its removal has no significant effect on the general failure behaviour of the composites of either fibre type. These findings stand in principal contrast to the observations by Hammell who described a general change in failure behaviour with apparent tensile failure in case of the desized carbon fibres [11].

The effect of the matrix strength on the properties of geopolymer matrix composites was shown in Table 5.5 and Figure 5.7. The results indicate that the use of the stronger M5 matrix has hardly any effect on the strength and the elastic modulus of the basalt fibre composites. On the other hand, the matrix composition appears to have significant impact on the properties of the carbon fibre composites. Although the seemingly higher average strength and modulus of the M5-carbon composites has to be relativized due to the higher fibre content of these samples compared to the M1-carbon composites (see Table 5.5), part of these improvements have to be attributed to the better matrix properties nonetheless. This apparent dependency of the importance of the matrix parameter on the particular fibre-matrix combination is not fully understood and may require further investigations. However, it is believed that the increased significance of the matrix strength is not specific to the carbon fibre composites but is a more general effect that becomes more important with increasing stiffness of the fibre. This aspect will also be discussed in some more detail below. In terms of failure behaviour, the change of matrix has some influence on the general stress-strain behaviour but does not change the actual failure mode observed for the M1-carbon and M1-basalt composites.

The issue of comparability between the results of different studies due to nonstandardised testing parameters for geopolymer matrix composites was initially discussed in chapter 2.3.1. This aspect was addressed in the present study by investigating the effect of two different testing parameters, namely the span-to-depth ratio and the overall specimen dimensions, on the mechanical properties and the failure behaviour of basalt fibre composites. The results were shown in Table 5.7, Figure 5.9 and Figure 5.10. As expected, the span-to-depth ratio has major influence 
on the measured strength value as well as the apparent failure mechanism. In terms of the overall sample dimensions, a decrease of the strength value can be expected with increasing sample size for a constant $s / d$ ratio. However, the overall sample size does not seem to have a major effect on the apparent failure mode observed for the composite for the same s/d ratio. These results clearly demonstrate how easily a simple change of testing parameters can lead to significantly different conclusions on the properties and failure behaviour of the same composite material. Therefore, the need for standardised testing methods for geopolymer matrix composites shall be emphasised again. That is not to say that the parameters applied here are necessarily the ideal parameters and the influence of other variables such as fabrication method, fibre content and fibre architecture (e.g. unidirectional or fabrics) may have to be taken into consideration, too. However, based on the present results, a minimum s/d ratio of $32: 1$ is recommended for the flexural testing of geopolymer matrix composites in order to reduce shear stresses and achieve tensile failure. Although smaller s/d ratios have been applied in previous studies as described in chapter 2.3.1 with different outcomes, the present results clearly indicate that smaller s/d ratios tend to induce predominantly shear failure. In case of the use of high-modulus fibres such as carbon and alumina, even higher $\mathrm{s} / \mathrm{d}$ ratios seem to be required to ensure the validity of the flexural test. However, the application of $\mathrm{s} / \mathrm{d}$ ratios $>32: 1$ may be limited by practical and economic reasons. Due to the apparent issues related to the flexural testing of geopolymer matrix composites, other testing methods such as a direct tensile strength test may be better suited for the characterisation of these materials.

For direct comparison of the influence of different loading directions on the composite properties, two $3 \times 3 \mathrm{~mm}$ samples were cut from the original basalt and carbon composite bars and tested for their out-of-plane and in-plane strength. The results of these tests were presented in Table 5.8, Figure 5.11 and Figure 5.12. The results clearly show the strong influence of the fibre type on the composite behaviour for the two different testing configurations. Surprisingly, the change of the loading direction has only very little effect on the strength of the basalt composites. This indicates a very homogeneous composite with good fibre-matrix and inter-layer 
bonding. On the other hand, the carbon composites show a more than $50 \%$ higher strength in the in-plane direction compared to the out-of-plane testing configuration. But even in the in-plane direction, no tensile failure of the carbon composites is observed. This behaviour is very untypical for ceramic composites subjected to inplane loading. Due to the prevention of premature interlaminar shear failure in the inplane loading direction, the in-plane flexural testing of ceramic composites generally results in tensile failure and fibre fracture. However, the current observations indicate that despite the much higher stress levels that can be achieved in the in-plane orientation, the fibres do not reach their maximum tensile stress. Instead, failure seems to occur due to matrix fragmentation in the interlaminar areas of the composite despite their orientation parallel to the loading direction. This results in the splitting of the individual fibre layers in proximity to the central loading point and the widening of the composite in that area as shown in Figure 5.12f). Hence, the matrix is the failure-dominating component under both loading directions for the carbon composites. These results also suggest that the matrix is essentially not strong enough to take full advantage of the properties of the carbon fibres. This, again, is believed to be an effect caused by the elastic mismatch between the fibre and the matrix rather than a specific phenomenon of the carbon fibre composite. Consequently, similar behaviour may also be observed for other fibre types above a certain elastic mismatch parameter.

\subsubsection{Failure behaviour of geopolymer matrix composites under flexural loading}

The flexural behaviour of geopolymer matrix composites is complex and includes a number of different failure modes. This makes the interpretation of the mechanical behaviour and the various stress-strain curves that were recorded for all composites difficult. Also, the transferability of classical failure concepts of composites, as discussed in chapter 2.1.3, is somewhat limited as these concepts typically originate from true tensile tests. Classical composite failure concepts were applied to describe the mechanical behaviour of geopolymer matrix composites in early studies of these materials $[3,11]$. However, the applicability of this concept to geopolymer matrix composites is very questionable. Given the weak nature of the geopolymer matrix, the weak matrix composite (WMC) concept seems much more applicable to describe 
the behaviour of geopolymer composites. But since the WMC concept was only developed in the mid-1990s, the knowledge of weak matrix composites would have been unavailable to these researchers, at least in the case of Foerster [3]. In any case, the WMC concept has been adopted by some researchers to describe geopolymer composite behaviour in more recent studies [13]. But despite some general failure studies, detailed fracture analyses of geopolymer composites are essentially not available at the current stage. In that regard, the investigations of the present study are also largely inadequate. However, a few general considerations, based on the observations made for the basalt, carbon and alumina composites, are discussed below. These considerations may form a basis for future investigations.

It was shown that the failure behaviour of geopolymer matrix composites is strongly influenced by the fibre type and the fibre properties. As discussed before, the fibre stiffness appears to be of particular importance in controlling the mechanical performance and the failure behaviour of geopolymer matrix composites. Due to the weak nature of the matrix, the fibre strength is of less importance for the composite behaviour. Under flexural loading, all three investigated composite types are characterised by an initial linear elastic region. For the basalt composites, a slight change in the slope of the initial region of the stress-strain curves can be observed between around $40-80 \mathrm{MPa}$. This effect is usually associated with the formation of microcracks within the matrix. However, the point of initial matrix microcracking is well above the tensile stress and strain capacities of the unreinforced matrix. Therefore, the incorporation of the basalt fibres appears to help to retard the formation of matrix cracks and effectively elevates the stress and strain levels at which matrix cracking occurs. This phenomenon is largely controlled by the stiffness of the fibre. Thus, even higher elevations of the stress level should be expected from the carbon and alumina fibres. The same general behaviour was described by Foerster [3]. For the case of a unidirectional carbon fibre composite, this author reported an elevation of the stress level, at which the first microcracking in the matrix occurred, to $>100 \mathrm{MPa}$. This is generally consistent with the observations made in the present study. However, the stress level of most carbon fibre composite samples seems to get increased close to the point of failure and a change in the slope 
of the stress-strain curve is hardly noticeable (see Figure 5.1). This is even more evident for the alumina fibre composites which essentially show linear elastic behaviour up to the point of failure. However, due to the lower strain capacity of the matrix compared to the fibres, matrix cracking would be expected in all cases according to the common understanding of composite theories. Nonetheless, the stress-strain curves do not indicate at which point the microcracking might occur. Thus, it is somewhat unclear if the high-modulus carbon and especially alumina fibres are in fact able to retard the microcracking of the matrix up to the apparent failure stresses of the composites or if the high modulus of the fibres conceals this feature in the stress-strain curves. It is also unclear, how the different extents of compression damage that are evident in all three composite types reflect in the stressstrain curves. In some cases the observation of minor fibre buckling is consistent with small bumps in the stress-strain curves. However, the considerable compression damage that is evident for the alumina fibre composites in form of a significant dent in the composite from the pressure of the loading pin as well as fibre fragmentation in the central compression area of the sample do not reflect in the stress-strain curves. Therefore, the interpretation of the stress-strain curves of geopolymer matrix composites under flexural loading give only limited information about the composite failure.

Whereas it appears that the mechanical behaviour of the basalt fibre composites can be described reasonably well by the existing WMC concept, there may be some question marks behind the applicability of this concept to adequately describe the behaviour of the carbon and alumina fibre composites. This thought shall be briefly explored below. The different behaviour of the three composite types is believed to be mainly a result of the different fibre stiffness and the increasing elastic mismatch of fibre and matrix, respectively. Referring back to the He-Hutchinson diagram in Figure 2.1, the principle of the weak matric composites evolves from the dependency of the relative fracture energy $\Gamma_{\mathrm{I}} / \Gamma_{\mathrm{F}}$ on the elastic mismatch between fibre and matrix. Thus, with increasing elastic mismatch parameter, a stronger interfacial strength can be accepted without inducing catastrophic failure. The maximum value of the elastic mismatch parameter for WMCs, as shown in Figure 2.1, is 0.85. This 
value is consistent with the elastic mismatch parameter for the basalt fibres and the geopolymer matrix. However, in the case of the carbon and alumina fibre composites the elastic mismatch parameter is significantly higher with values of 0.92 and 0.95 , respectively. In addition, the interfacial strength is rather low and cannot be adjusted through the matrix porosity, as is the case in porous ceramic composites. Thus, in the case of the carbon and alumina composites we have a material with an extremely high elastic mismatch between fibres and matrix and a low interfacial strength. It is suggested that for this combination, the matrix is essentially too weak to take full advantage of the high modulus fibres and that matrix failure will always occur under flexural loading before the maximum stress level of the fibre is reached. This may be most evident from the results of the in-plane test of the carbon fibre composites. Due to the elimination of interlaminar shear failure, the in-plane loading configuration typically results in fibre fracture. But despite a significant increase of the apparent flexural stress, matrix failure occurred even under this loading orientation before the critical fibre stress was reached. Therefore, it is proposed that in addition to a maximum boundary criterion for the relative fracture energy, also a minimum criterion for the interface and the matrix strength may be required for a particular fibre-matrix combination in order to take full advantage of the fibre properties. In extension to the weak matrix composites concept, these materials may be considered as "very weak matrix composites". 


\section{Chapter 6}

\section{Fibre-matrix interactions}

The microstructural aspects of the composite samples, in particular the interaction between the different fibre types and the matrix (composition M1) at the fibre-matrix interface, were studied on fractured and polished surfaces by means of electron microscopy and EDS analyses. Powder x-ray diffraction (XRD) analysis was used to identify any crystalline phases in the composites.

\subsection{Fibre Fibre-matrix interaction at room temperature}

\subsubsection{Chemical stability and desizing of fibres}

In order to strengthen and toughen geopolymer matrices effectively, the reinforcing fibres are required to remain chemically stable, i.e. not degrade under the present environmental conditions of the composite fabrication process. For geopolymer matrix composites, the main factor that may limit the use of some fibres is corrosive attack under the highly alkaline conditions required for the geopolymer synthesis. Therefore, the chemical stability of all fibre types (see Table 3.2) was tested in a preliminary experiment in a concentrated $12.5 \mathrm{M} \mathrm{NaOH}$ solution for $150 \mathrm{~h}$ at $40^{\circ} \mathrm{C}$ to evaluate the general applicability of these fibres in geopolymer composites. The exposure to the alkali solution caused the removal of the fibre sizing of all sized 
fibres, as shown in Figure 6.1 on the example of basalt fibres, leaving a clean fibre surface. SEM analysis revealed no signs of any form of corrosive attack on the actual fibre surface for any type of fibre.
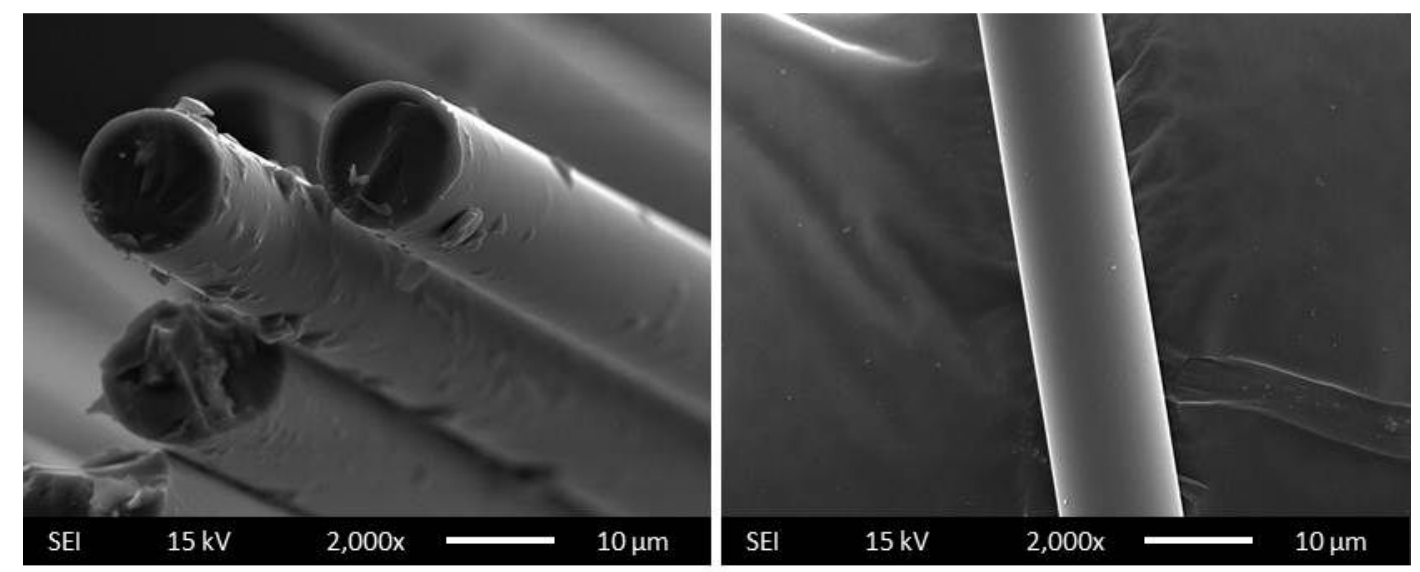

Figure 6.1: SEM micrographs of basalt fibres before (left) and after (right) exposure in concentrated $\mathrm{NaOH}$ solution at $40^{\circ} \mathrm{C}$ for $150 \mathrm{~h}$. No form of corrosive attack on the fibre surface is observed. The removal of fibre sizing is typical for all investigated types of sized fibres

Carbon and Nextel 610 alumina fibres were desized using a Bunsen burner. SEM analysis confirmed that this process could successfully remove the fibre sizing without causing any notable degradation of the fibre surfaces. This is of particular importance for the carbon fibres which generally start to show significant fibre degradation and decomposition at temperatures around $600^{\circ} \mathrm{C}$. Although some loose outer fibres of each carbon fibre tape appeared to burn off during the desizing process the majority of the fibre bundle remained unaffected probably due to the short time that the fibres were exposed to the heat of the Bunsen burner.

\subsubsection{Microstructural analysis of geopolymer composites}

The fracture surfaces of fibre composite samples showed significant differences in the adhesion between matrix and fibre, depending on the fibre type. This is shown in Figure 6.2. The basalt fibres generally displayed a largely clean fibre surface with a sparse distribution of very fine particles attached to it. The carbon fibres, both sized and desized, showed essentially no interaction between the fibre and the matrix. The best wetting and adhesion between matrix and fibre was observed in Nextel 610 alumina fibre composites. Most alumina fibres were found to be almost completely 
covered by a layer of geopolymer matrix particles. The thickness of the matrix layer varied but typically ranged on a scale of several hundred nanometres. Differences were also observed between the sized and desized Nextel alumina fibres. The desized fibres generally showed better fibre-matrix adhesion and a thicker matrix layer surrounding the fibres. However, not all of the fibres were covered by a matrix layer. Several fibres showed very clean surfaces without any apparent change to the original fibre surface morphology. Figure 6.3 shows a Nextel 610-DS fibre at the transition point between the two surface appearances. The abrupt change between the heavily covered top half and the relatively clean bottom half of the fibre is clearly visible.
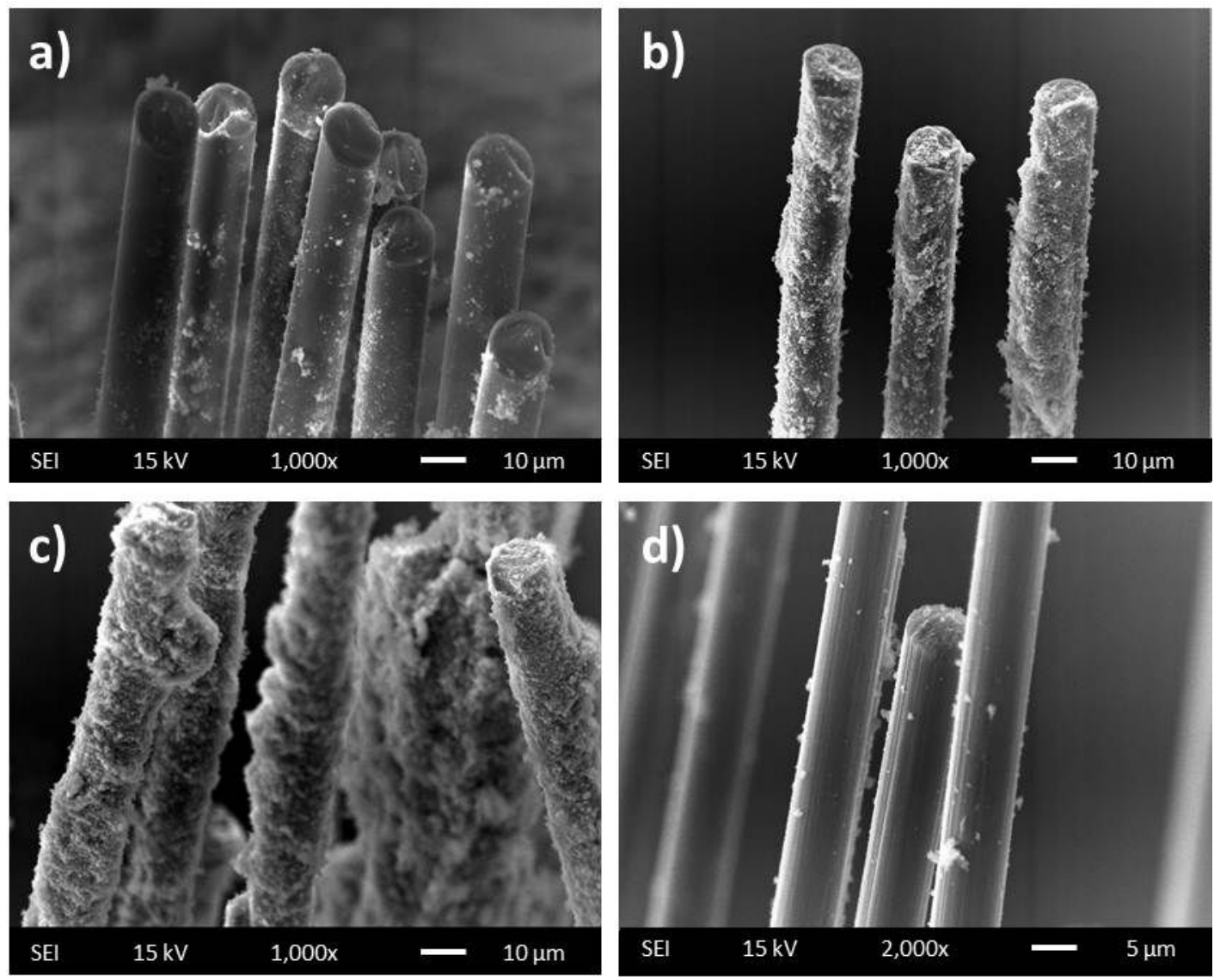

Figure 6.2: Adhesion between M1 matrix and a) basalt, b) Nextel 610, c) Nextel 610-DS and d) carbon/carbon-DS fibres 

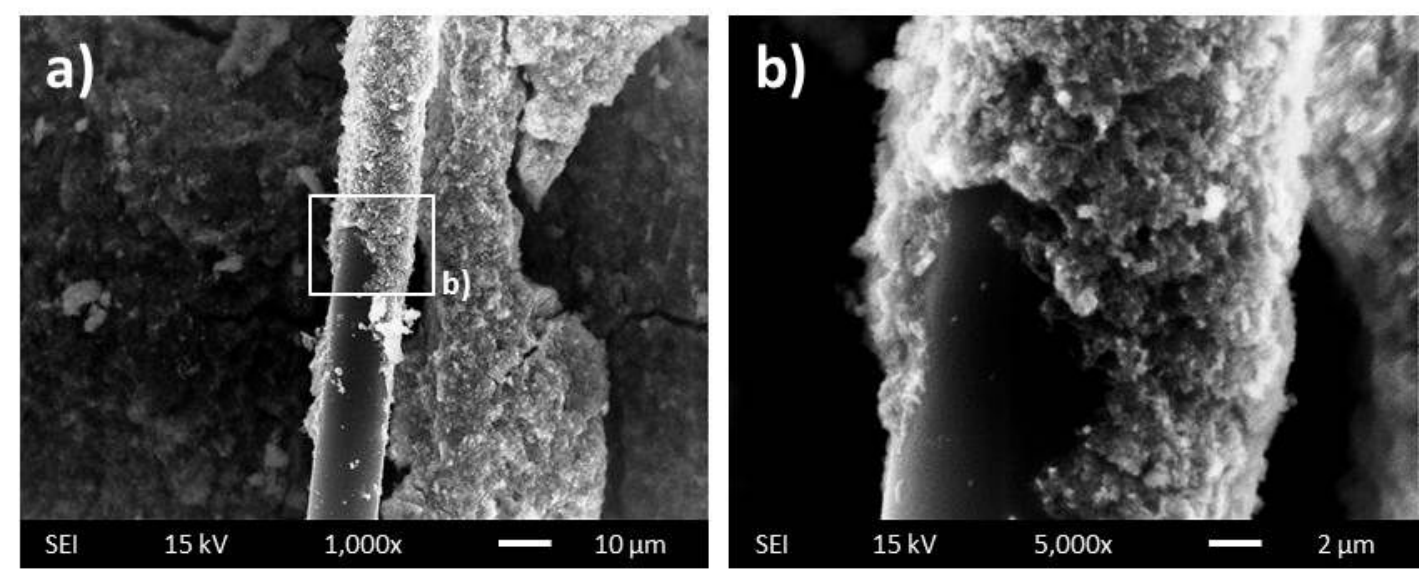

Figure 6.3: Nextel 610-DS fibre at the transition point between a thick matrix adhesion layer and clean fibre surface

The preparation of reasonably good surface finishes of unheated composite samples was somewhat difficult due to the large mismatch between the properties of the fibres and the matrix. The higher abrasion rate of the comparatively soft matrix caused the fibres to slightly stick out and agglomeration of ground matrix material around the fibres often caused smeared and blurry fibre edges (see SEI images in Figure 6.4 and Figure 6.5). Nonetheless, the formation of an interfacial reaction layer was not evident for any of the three fibre types by SEM imaging. Since the inspection of both fractured and polished samples of the carbon fibre composites showed no indication of any interfacial interaction, no further analyses were carried out for these composites. Additional EDS mapping and line scan analyses were performed on the basalt and alumina fibre composites. However, the EDS analyses also did not reveal any significant degree of interaction between the two components. The EDS line scan profiles across the fibre-matrix interface of a basalt and alumina fibre composite sample are shown in Figure 6.4 and Figure 6.5, respectively. Whereas most element profiles for the basalt fibre composite in Figure 6.4 showed a relatively abrupt increase or decrease of the intensity in close proximity to the fibre edge, the sodium and potassium profiles displayed a somewhat gentler change of intensity across the fibre-matrix interface. The EDS line scan profiles for the alumina fibre composite were also characterised by a very sudden change of the element concentration across the interface. It should be noted that the EDS line scan profile in Figure 6.4 as well as subsequent EDS line scan profiles are presented in a purely 
qualitative way, showing the relative change of the counts or intensity for each element along the indicated line. Thus, they do not provide any information about the absolute element concentration.
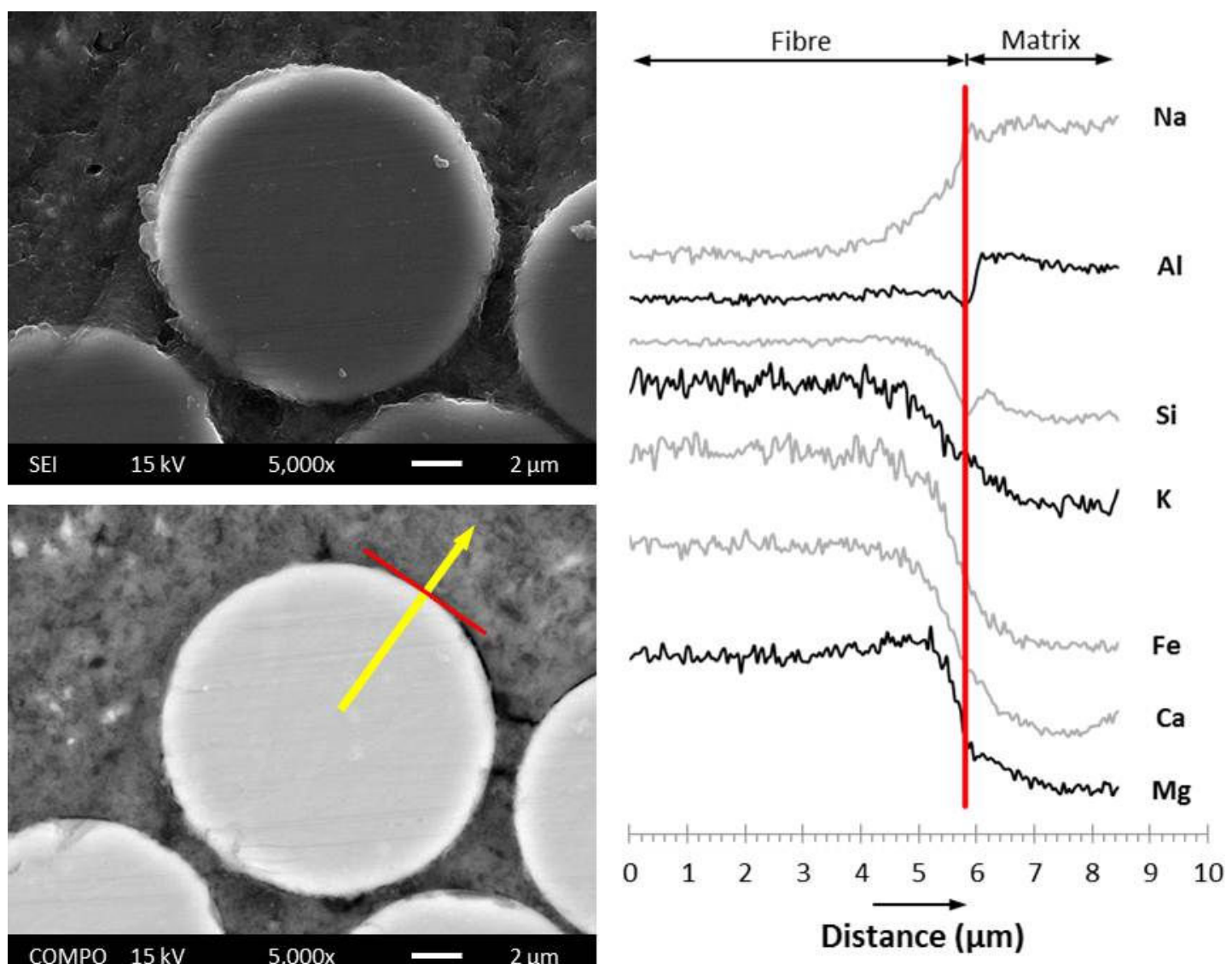

Figure 6.4: SEI and backscattered images of the polished surface of the M1-Basalt composite and corresponding EDS line scan profile across the fibre-matrix interface

$\mathrm{XRD}$ analysis did not indicate the formation of any crystalline phases as a result of fibre-matrix interaction in any of the unheated composite types, consistent with the observations from the SEM analyses. As can be seen from Figure 6.6, the XRD pattern of the basalt composite is essentially a combination of the diffractograms of the individual components. 

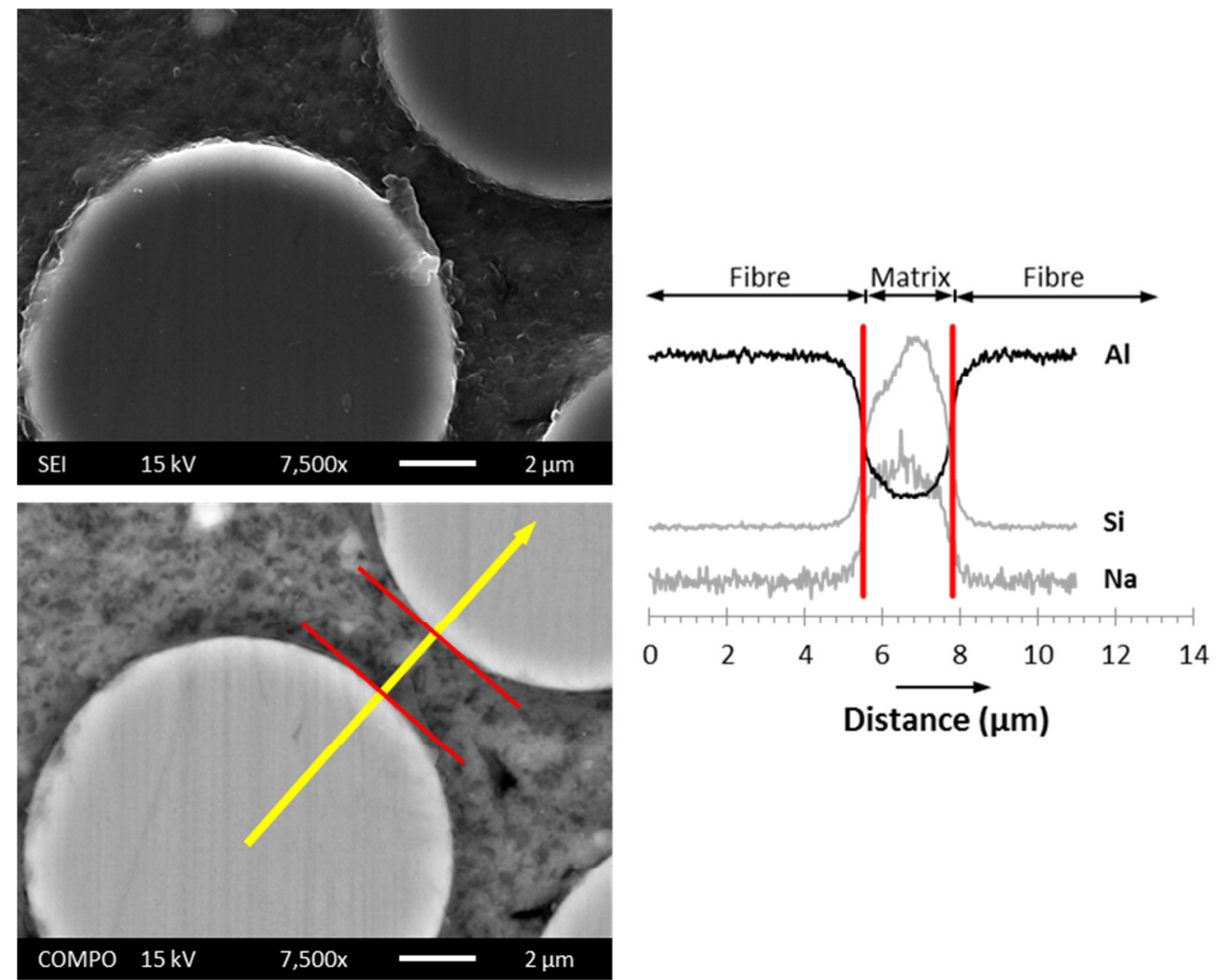

Figure 6.5: SEI and backscattered images of the polished surface of the M1-N610 composite and corresponding EDS line scan profile across the fibre-matrix interface

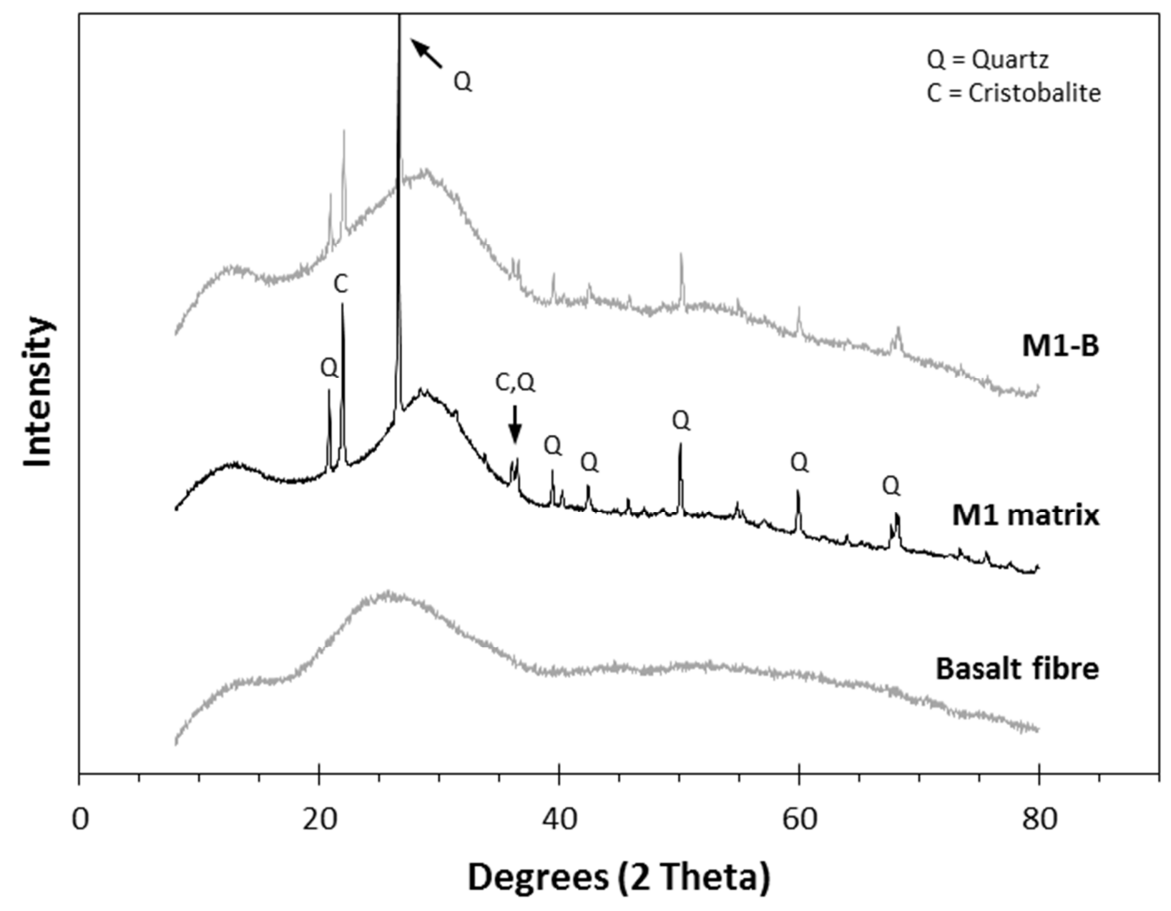

Quartz (PDF 01-089-8935)

Cristobalite (PDF 04-007-2134)

Figure 6.6: X-ray diffraction patterns of basalt fibre, M1-Basalt composite and M1 reference sample 


\subsubsection{Discussion}

The results of the fibre corrosion test indicate that all of the investigated fibres are stable under highly alkaline conditions and are therefore, in principal, suitable for the use in geopolymer matrix composites. The microstructural investigation of the basalt, carbon and alumina fibre composites did not indicate any significant interfacial reaction between the fibres and the geopolymer matrix at room temperature. Only the EDS line scan analysis of the basalt fibre interface indicated some degree of interaction in the form of interdiffusion of the two alkali ions sodium and potassium, see Figure 6.4. In the absence of any apparent interfacial reaction for any of the three fibre types, the fibres can be assumed to be only mechanically bonded in the matrix. Thus, without changing any other parameter, differences in the interface strength mainly arise from the chemical nature of the fibre and its wettability by the geopolymer binder. As shown in Figure 6.2, the adhesion of a relatively thick matrix layer on large parts of the alumina fibre surfaces indicates a very good wettability and good interfacial strength for this fibre type. The carbon fibres, on the other hand, show no adhesion of matrix on the fibre surface indicating bad wettability and a weak interfacial strength within the composite.

The initial corrosion test also revealed the removal of the fibre sizing from the fibre surface under the apparent conditions in all cases. Since the geopolymer binder is prepared under similar high-alkaline conditions, the dissolution of the fibre sizing is also suspected to occur in the composites. However, it is less clear what exactly happens to the fibre sizing in the composite and how the reaction between fibre sizing and geopolymer binder affects the properties of the fibre-matrix interface or the matrix itself. The electron microscopic analysis of polished surfaces revealed no information in that respect. Analysis of fractured surfaces, as shown in Figure 6.2, revealed that the fibre sizing of the alumina fibres has some effect on the adhesion of matrix on the fibre surface resulting in a thicker matrix layer in the case of the desized fibres. However, clean fibre surfaces without any adhering matrix were observed in other areas for both sized and desized alumina fibres. Thus, the consequence of the different thickness of the matrix layer adhering to the alumina fibre surface and the role of the fibre sizing is not clear. Especially since the sized 
alumina fibre composites, which showed a lower amount of matrix adhering on the fibre surface, achieved a similar if not even slightly higher strength compared to the composites made with desized fibres. In the case of the carbon fibres, the SEM analysis revealed no apparent difference in the microstructural appearance of the sized and desized carbon fibre composites that could explain any change in the composite strength. Thus, the role of the fibre sizing can only be speculated upon. Since no obvious remnants of the original fibre sizing were seen on the fibre surfaces of all three main fibre types, the fibre sizing most likely dissolves into the geopolymer binder due to its high $\mathrm{pH}$ and alkalinity. As discussed in the previous chapter 5, the effect of the fibre sizing on the mechanical properties of the composites seems relatively small. Nevertheless, further investigations of this aspect seem necessary to help predict the potential effects of fibre sizings on the mechanical properties of geopolymer matrix composites. Based on the present results, there seems to be no stringent need for the desizing of fibres for their use in geopolymer matrix composites, at least for room temperature applications. However, the role of the fibre sizing may become more important at higher temperatures. This aspect will be discussed further on in this chapter.

\subsection{Fibre-matrix interaction at elevated temperatures}

The thermal behaviour of the composites was studied by the heating of small composite bar pieces to 600,800 and $1000^{\circ} \mathrm{C}$ for $1 \mathrm{~h}$ in air by means of SEM, EDS and XRD analyses.

\subsubsection{Basalt fibre composites}

The composite samples heated to $600^{\circ} \mathrm{C}$ showed only little difference to the unheated composites on a macroscopic level. Some small grey-black areas on the sample surface were evidence of the incomplete burnout of the fibre sizing. Although a slight embrittlement of the composite sample was noted, the fracture behaviour remained largely unchanged and was characterised by matrix fragmentation and some extent of fibre fracture. Thus, the fibres had to be cut in order to obtain a 
fracture surface suitable for SEM analysis. Heat treatment at higher temperatures caused increasing embrittlement of the fibres and the composite, resulting in brittle failure of composite samples heated to 800 and $1000^{\circ} \mathrm{C}$. Low magnification SEM images of the fracture surfaces of the heated samples are shown in Figure 6.7. The image of the (cut) fracture surface of the $600^{\circ} \mathrm{C}$ sample in Figure 6.7a) is dominated by long debonded fibre segments. The fracture surface of the brittle $800^{\circ} \mathrm{C}$ sample in Figure $6.7 \mathrm{~b}$ ) is relatively uneven but shows several smaller planar fracture levels with strongly bonded fibres in the surrounding matrix. A large planar fracture surface without any apparent fibre pull-out was observed for the basalt composite heated to $1000^{\circ} \mathrm{C}$, clearly indicating the brittle failure of the composite sample (Figure 6.7c). A colour change of the composite samples was also observed after heating to the different temperatures. The unheated composite had a marbled green colour resulting from the naturally green colour of the basalt fibre and the white colour of the matrix. This appearance hardly changed up to $600^{\circ} \mathrm{C}$. However, the colour appearance of the composite changed from green to black and from black to orange-brown for samples heated to 800 and $1000^{\circ} \mathrm{C}$, respectively. Similar colour changes were observed for the heated basalt fibres alone. Therefore, the colour change is a result of chemical reactions within the basalt fibre rather than being caused by interaction with the geopolymer matrix. The white colour of the matrix was not affected by the colour change of the fibres and was still visible in matrix-rich areas of the heated composite samples. It was also evident from the heating of the basalt fibres alone that not just the composite but also the fibre itself is subject to increasing embrittlement with increasing temperature. 

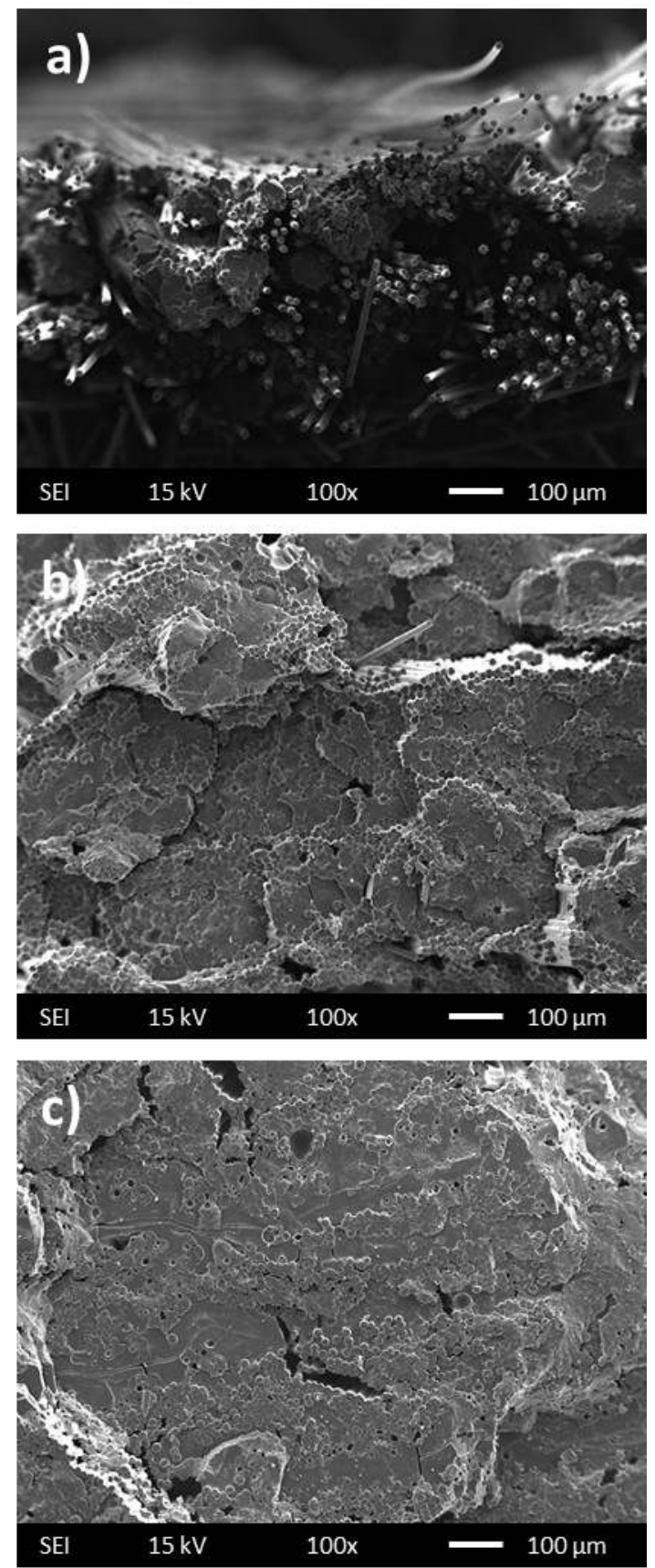

Figure 6.7: Fracture surfaces of basalt fibre composites after heat treatment at a) $600^{\circ} \mathrm{C}$, b) $800^{\circ} \mathrm{C}$ and c) $1000^{\circ} \mathrm{C}$

A closer analysis of the fractured surface of the $600^{\circ} \mathrm{C}$ sample showed that most of the loose, debonded fibres were covered by a small amount of irregularly shaped and sized matrix fragments similar to the unheated composite. These fragments appeared to be only loosely attached to the fibre surface and are considered artefacts of the fragmented matrix. The fibre surfaces in areas where the matrix did not completely 
fracture around the fibres appeared to be very clean and unreacted. Figure 6.8a) shows a basalt fibre embedded in the matrix on a fracture surface along the fibre orientation. The smooth and unchanged surface morphology of the fibre and the neighbouring empty fibre channel are clearly visible. The fibre imprint reveals a good view of the matrix structure at the immediate vicinity of the fibre, see Figure $6.8 \mathrm{~b})$. The structure appears to consist of a homogeneous densely packed layer of nearly spherical particles with a size of roughly $100 \mathrm{~nm}$. The microstructural appearance of the samples heated to $800^{\circ} \mathrm{C}$ remained essentially unchanged. Therefore, the micrographs in Figure 6.8 can be considered equally representative for samples heated to both temperatures.
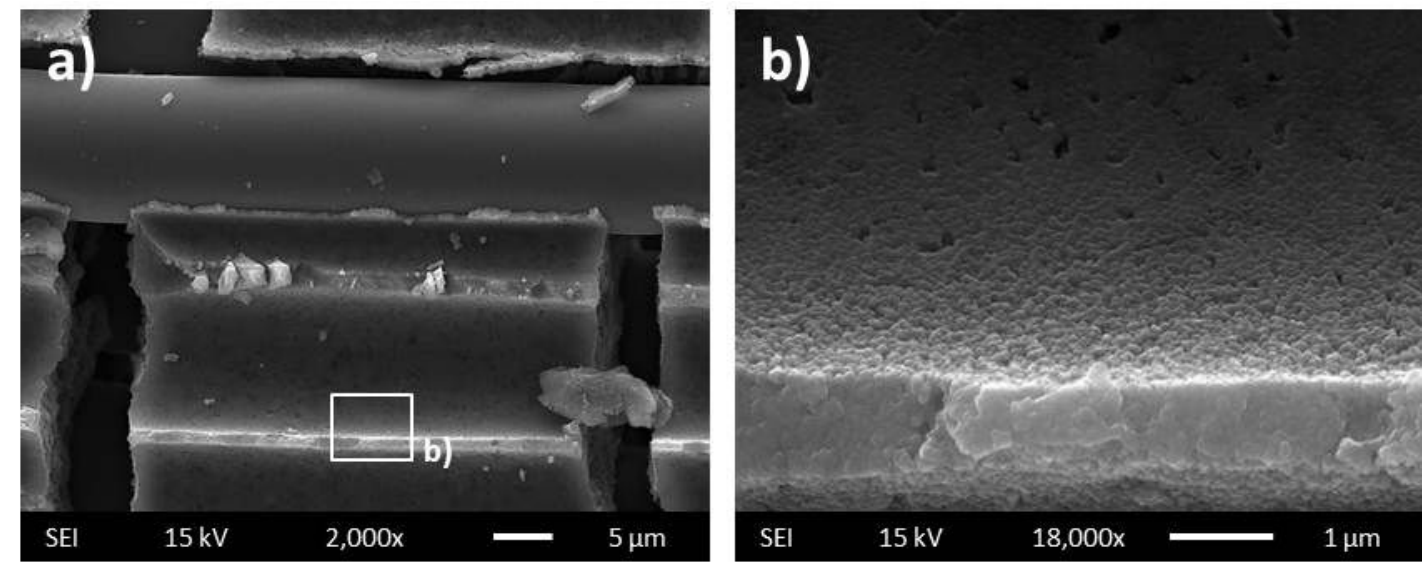

Figure 6.8: $\mathrm{SEM}$ micrographs of $\mathrm{M} 1$-Basalt composites heated to $800^{\circ} \mathrm{C}$ showing an embedded fibre in the fractured composite and the matrix microstructure in an empty fibre channel. The microstructural appearance displayed in both images is equally representative for the $600^{\circ} \mathrm{C}$ samples

$\mathrm{XRD}$ analysis of the basalt composite heated at $600^{\circ} \mathrm{C}$ showed only slight changes compared to the unheated sample and no indication of any crystallisation reaction at that temperature, see Figure 6.9. A shift of the amorphous hump and a decrease of the intensity of the quartz and cristobalite peaks were noticeable for the heated sample. 


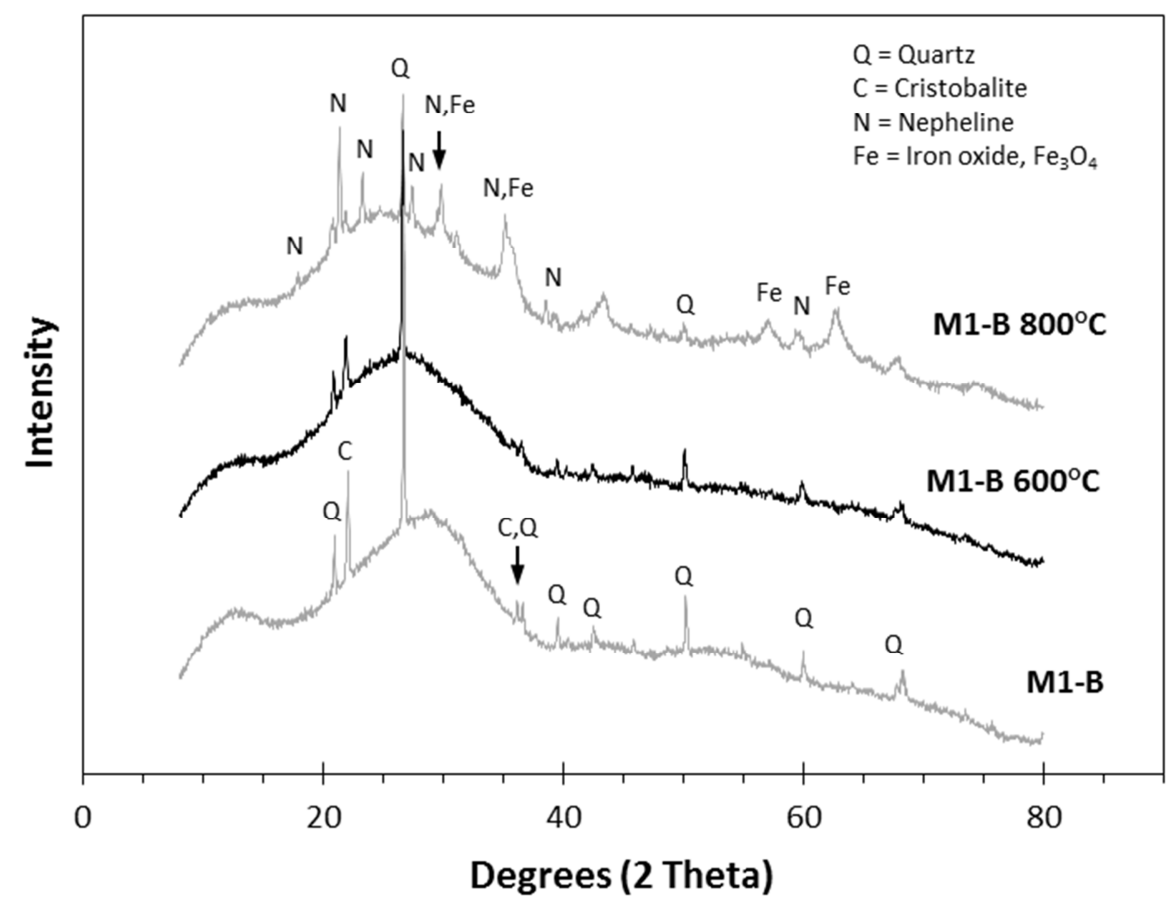

Quartz (PDF 01-089-8935) Cristobalite (PDF 04-007-2134)

Nepheline (PDF 04-012-4977) $\quad \mathrm{Fe}_{3} \mathrm{O}_{4}$ (PDF 04-013-9811)

Figure 6.9: X-ray diffraction patterns of M1-Basalt composites heated to 600 and $800^{\circ} \mathrm{C}$ for $1 \mathrm{~h}$

The macroscopic colour change from green to black of the basalt composite sample after heating to $800^{\circ} \mathrm{C}$ was accompanied by microstructural changes in the fibre. Although the basalt fibres appeared unchanged under standard SEM imaging conditions, the formation of a reaction zone along the inner fibre edge of most fibres was revealed in backscattered mode, see Figure 6.10. However, the characteristic of this reaction zone was less pronounced for some fibres than for others. Figure 6.10b) shows a typical approximately $2 \mu \mathrm{m}$ wide reaction zone and what appears to be the formation of nano-sized crystallites. The corresponding EDS line scan profile indicates the concentration of iron in the reaction zone but also considerable interdiffusion between the fibre and the matrix, in particular for sodium and calcium. According to the EDS line scan profile, the calcium diffuses from the fibre into the matrix whereas the sodium diffuses from the matrix into the fibre. XRD analysis confirmed the formation of crystalline iron oxide $\mathrm{Fe}_{3} \mathrm{O}_{4}$ (magnetite) as well as nepheline in the composite after heating to $800^{\circ} \mathrm{C}$ (Figure 6.9). 

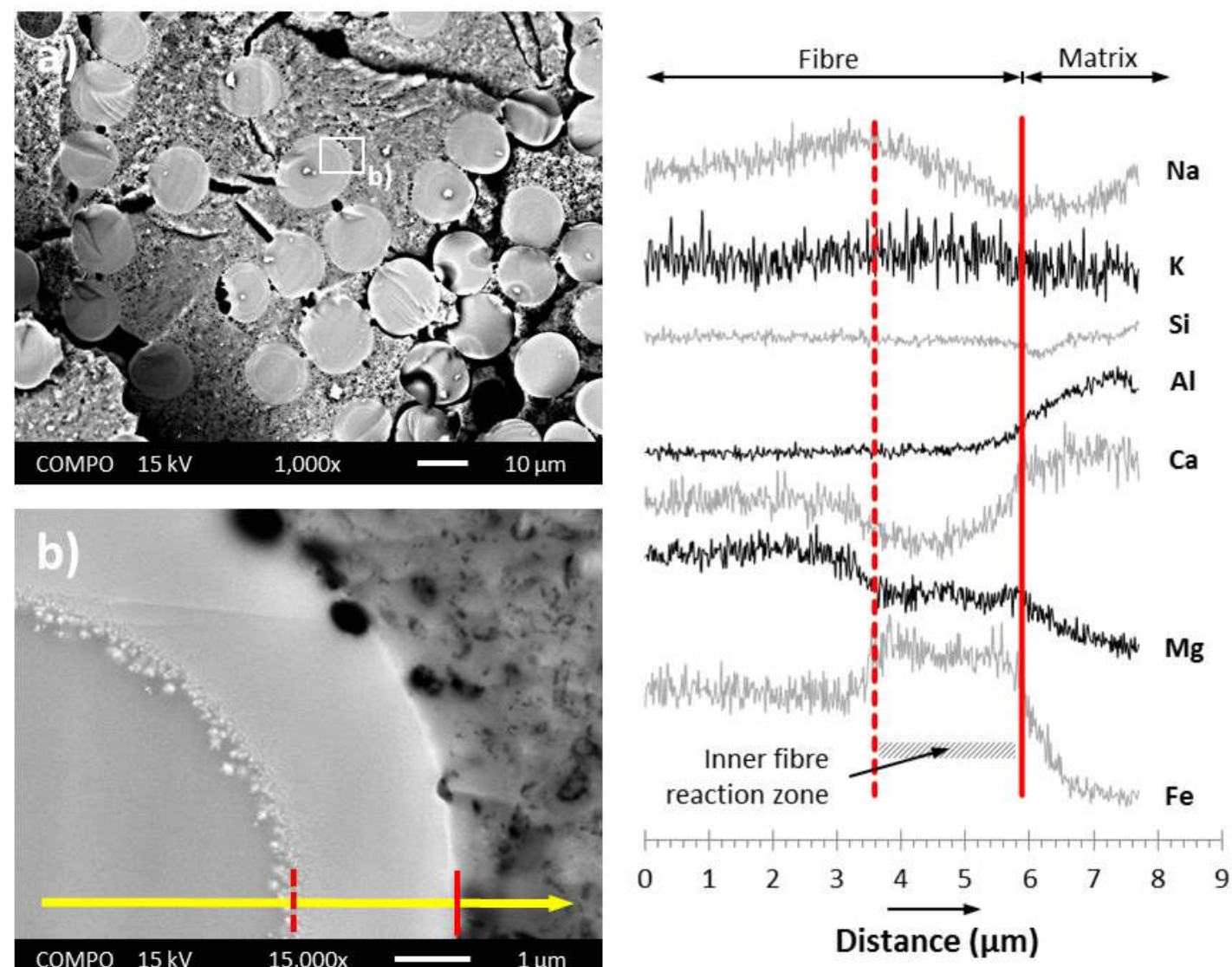

Figure 6.10: SEM backscattered images of the fracture surface of the M1-Basalt composite heated at $800^{\circ} \mathrm{C}$ for $1 \mathrm{~h}$. EDS line scan analysis indicates the formation of an iron-rich inner fibre reaction zone

With increasing densification of the matrix at higher temperatures the accumulation of pores along the fibre-matrix interface and subsequent migration into the fibres was observed. Both images in Figure 6.10 show small pores around the fibre edges that are starting to penetrate the fibres at $800^{\circ} \mathrm{C}$. The smaller pores continued to migrate into the centre of the fibres and at $1000^{\circ} \mathrm{C}$ most of these pores were found to have combined into single larger pores inside the fibres. Examples of this can be seen in Figure 6.11a) and Figure 6.12a). However, the heating of the basalt composite samples did not result in a homogeneous densification of the matrix. Whereas major densification of the matrix was evident in some areas, the formation of very porous matrix structures was also observed. An example of a random porous area embedded in a much denser surrounding matrix is shown in Figure 6.11a).

Significant deformation of the round-shaped fibres was observed after heating of the basalt composite to $1000^{\circ} \mathrm{C}$. The typical appearance of the fibres at the fracture 
surface of the composite is shown in Figure 6.11b). Figure 6.12 shows that, in particular in densely packed fibre areas, the fibre deformation was accompanied by a marked shape accommodation of the surrounding fibres. The polished samples revealed the formation of three relatively distinct reaction zones within the basalt fibre for most fibres. However, the characteristics of each zone varied to considerable degrees (see for example Figure 6.12). Common to all fibres was the formation of an approximately $200 \mathrm{~nm}$ wide reaction zone along the inner fibre edge which appeared as a bright line in the backscattered micrographs. Crystallisation was evident in the centre of the fibres. However, the form and shape of crystallites that formed in the centre of the fibres varied significantly from one fibre to another, as can be seen for example in Figure 6.12 and Figure 6.14. The size of the crystallites ranged from the low nanometre scale up to around $1 \mu \mathrm{m}$. The outer and inner reaction zones were separated by a $\sim 2 \mu \mathrm{m}$ wide band. A fine distribution of tiny bright spots was revealed by high resolution backscattered imaging, indicating that this zone was also subject to some form of precipitation or crystallisation reaction. However, in contrast to the inner fibre zone, this zone showed similar appearance in all fibres.
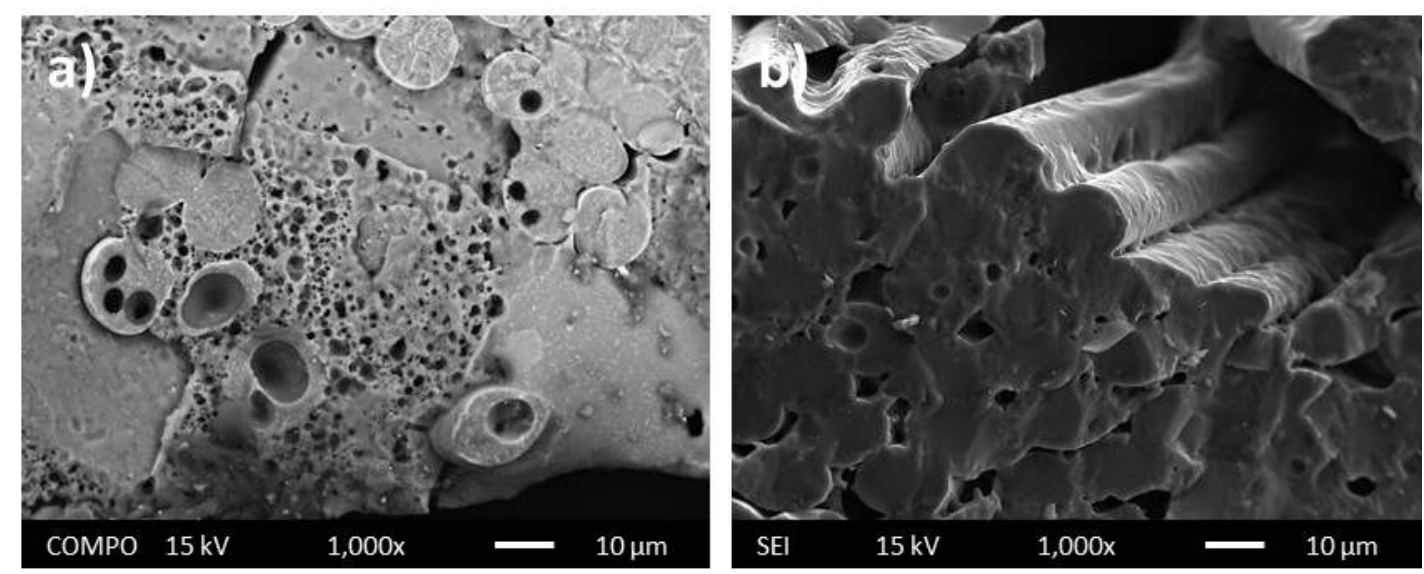

Figure 6.11: SEM images of fracture surfaces of the M1-Basalt composite after heating to $1000^{\circ} \mathrm{C}$. The images show the formation of large pores within the fibre; inhomogeneous, dense and porous matrix areas and fibre deformation within the composite

The EDS line scan profile in Figure 6.12 and the corresponding EDS elemental maps in Figure 6.13 indicate that a significant percentage of the present calcium diffused into the matrix. The sodium, on the other hand, diffused from the matrix into the 
outer parts of the fibre up to about $\sim 4 \mu \mathrm{m}$ from the fibre edge whereas the potassium content was low in that area compared to the fibre centre. The behaviour of the iron and magnesium profiles was relatively similar to the one observed at $800^{\circ} \mathrm{C}$, at least for the fibre on the right hand side of the line scan, indicating a higher concentration of iron around the fibre edges. The concentration of silicon was nearly constant across the fibre-matrix interface. Some more information about the elemental composition of the outer reaction zone of the fibres could be obtained at higher magnification as shown in the micrograph in Figure 6.14. It is evident from the EDS line scan profile that the outermost reaction zone of the fibre showed a peak concentration of iron and also an elevated magnesium content. However, comparison of the full EDS elemental maps of the two micrographs in Figure 6.13 and Figure 6.14 shows that the element distribution as described above was not consistent
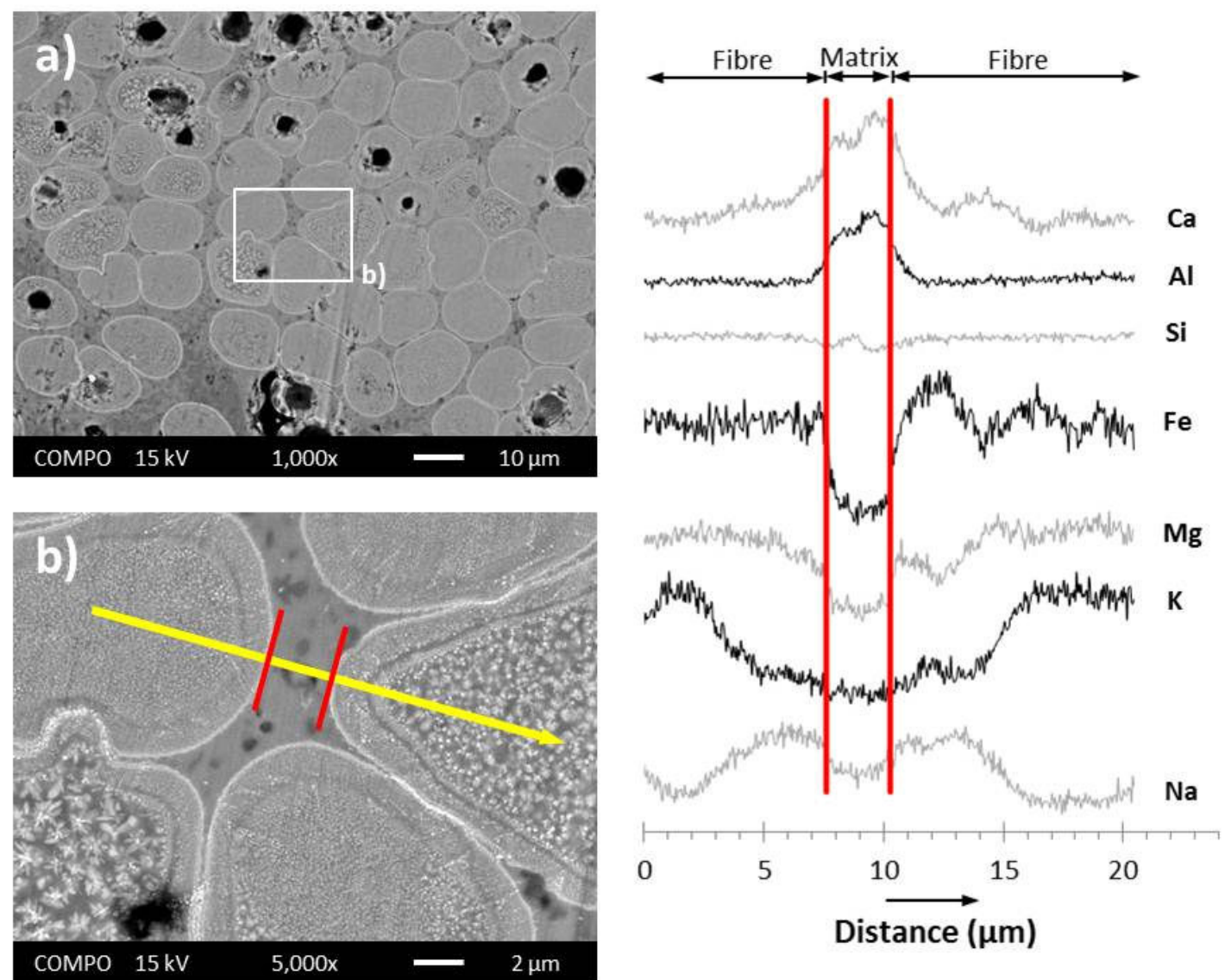

Figure 6.12: SEM backscattered images of the polished surface of the M1-Basalt composite after heating to $1000^{\circ} \mathrm{C}$ showing fibre shape accommodation and crystallisation within the fibre and EDS line scan profile across the fibre-matrix interface (see also Figure 6.13 for EDS elemental maps) 

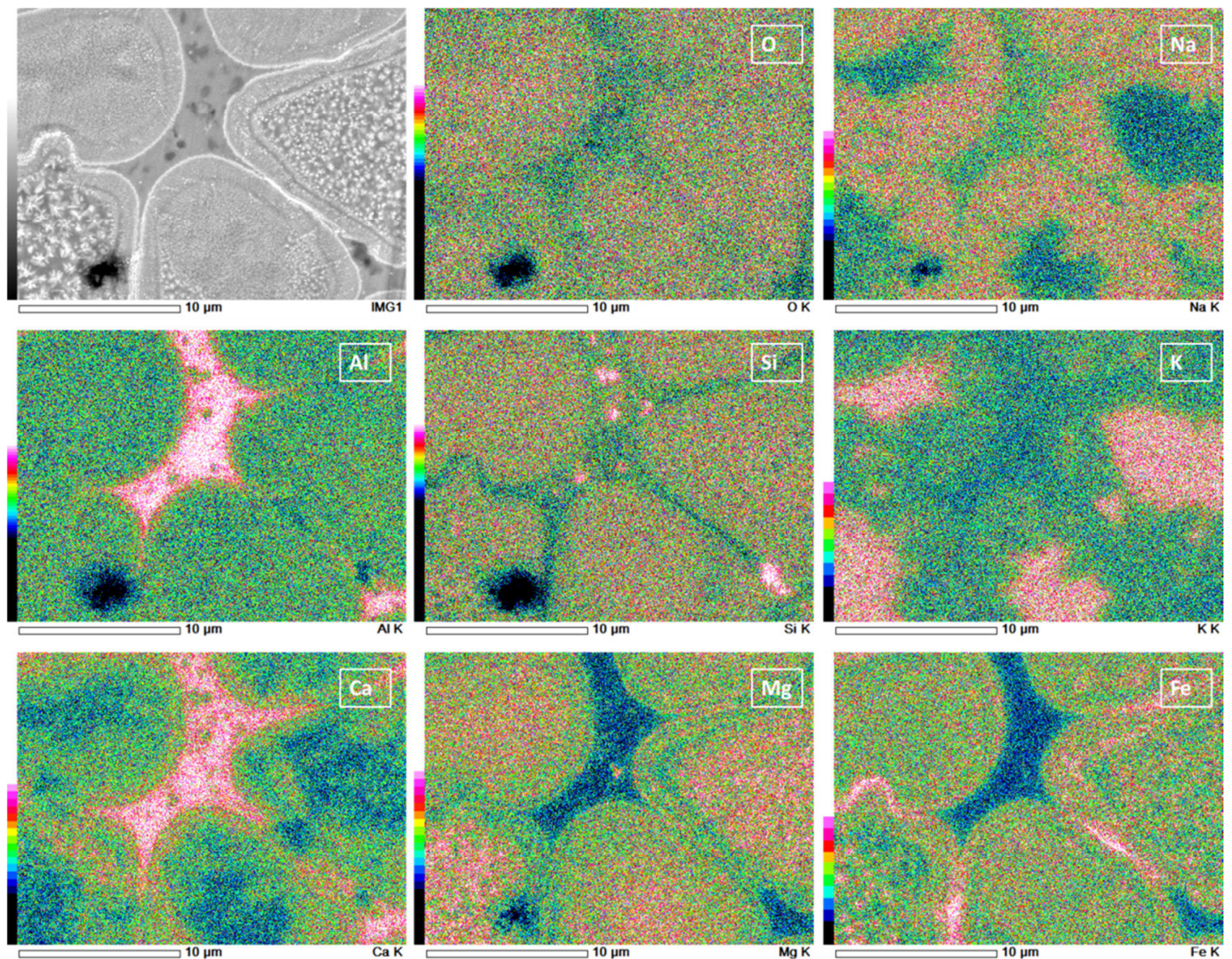

Figure 6.13: EDS elemental maps corresponding to micrograph shown in Figure 6.12(b) (element concentration increases from dark to bright colour)

throughout the composite. Whereas the formation of at least three distinct reaction zones within each fibre as well as the concentration of iron along the fibre edges was largely consistent for both micrographs, the distribution of the other elements seemed less consistent. In particular the concentration of the $\mathrm{Na}, \mathrm{K}$ and $\mathrm{Ca}$ in the two micrographs in the respective inner-fibre reaction zones and the matrix seems somewhat contradictory. Therefore, no clear conclusion on the elemental distribution within the different reaction zones can be drawn.

The merging of two fibres and the penetration of the inner phase of one fibre into the other, as shown in Figure 6.14, was very rare. Although the indenting of one fibre into a neighbouring fibre was not untypical, the boundary between the two fibres was usually clearly marked by the outer reaction zone of the fibres, see Figure 6.12. 

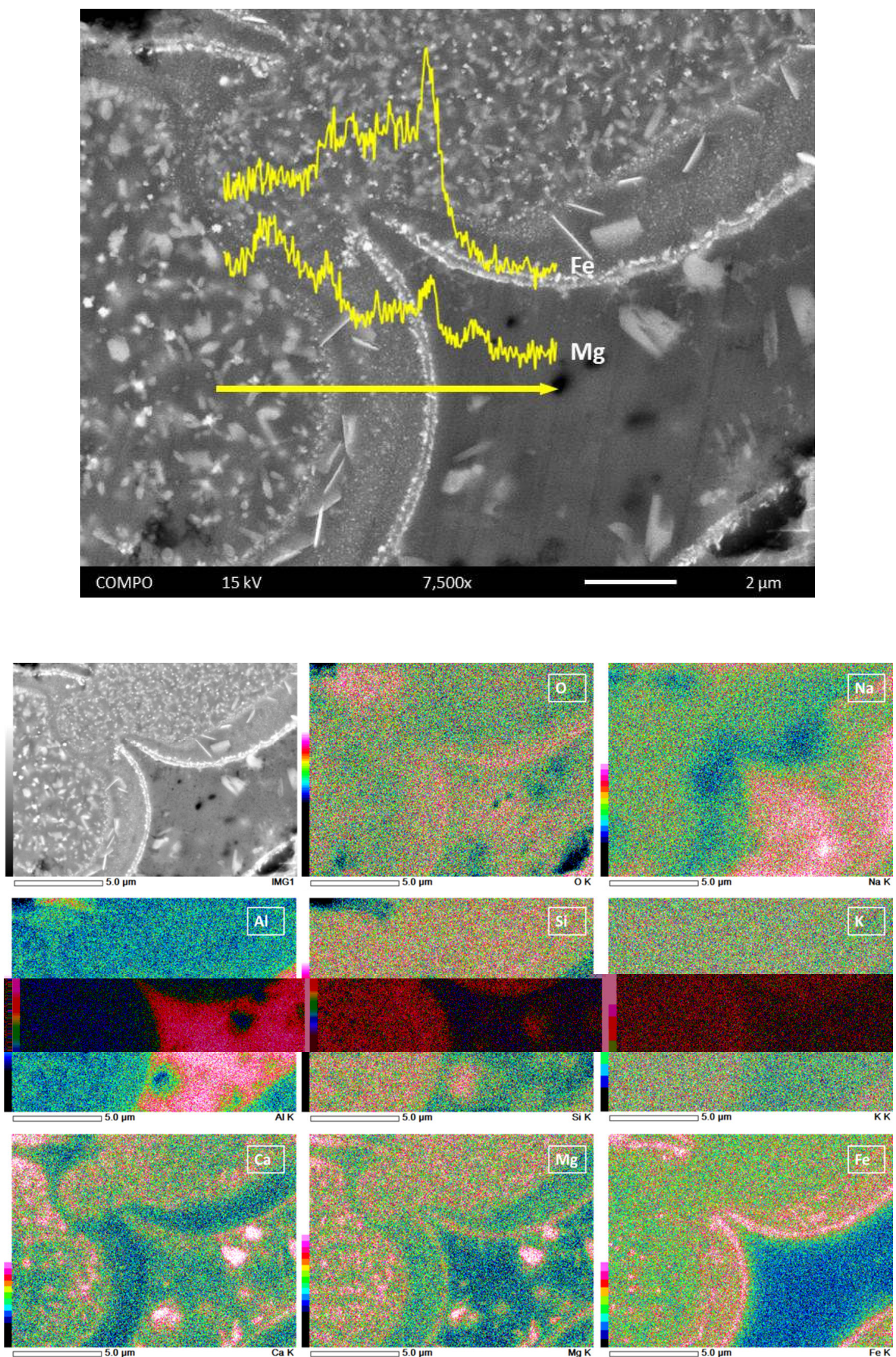

Figure 6.14: Backscattered SEM image of fibre-matrix interface of the M1-Basalt composite after heating to $1000^{\circ} \mathrm{C}$ at higher resolution with overlay of EDS line scan profile for $\mathrm{Fe}$ and $\mathrm{Mg}$ and corresponding EDS elemental maps (element concentration increases from dark to bright colour) 
Since most of the reactions in the heated basalt composites seemed to occur within the fibre, a bundle of loose basalt fibres was also heated to $1000^{\circ} \mathrm{C}$ in order to compare the reaction in the fibre without the geopolymer matrix. A micrograph of the cross section of the fractured fibre bundle is shown in Figure 6.15. The image shows that only a very small number of fibres resembled the approximate reaction pattern of the fibres in the composite sample. Although the formation of nanoprecipitates especially along the fibre edges was evident at higher magnification for all fibres, the formation of an outer reaction zone similar to the one observed in the composite sample was insinuating at best. A slight deformation of the fibre cross section and the formation of sinter-necks between fibres were also clearly visible. However, along the fibre length, the surface of the basalt fibres appeared smooth and even unlike the fibres in the composite as shown in Figure 6.11b).

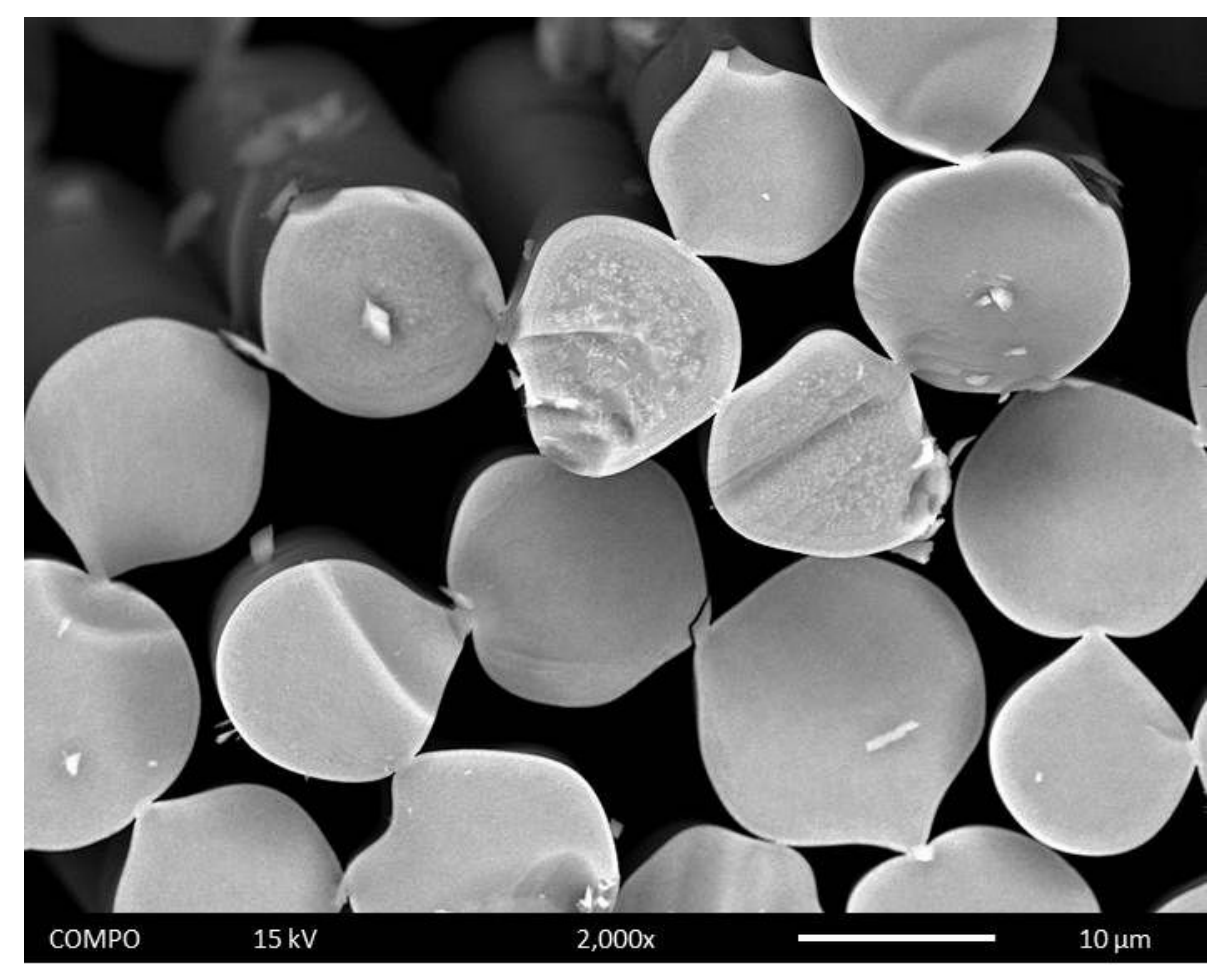

Figure 6.15: Backscattered SEM image of fractured basalt fibres heated to $1000^{\circ} \mathrm{C}$ for $1 \mathrm{~h}$

The $\mathrm{x}$-ray diffractogram of the basalt composite heated to $1000^{\circ} \mathrm{C}$ was complex and indicated the formation of a number of different crystalline phases. The XRD patterns of the composite as well as the diffractograms of the heated matrix and basalt fibre alone are displayed in Figure 6.16. As mentioned before, the matrix 
showed nearly complete crystallisation with nepheline as the sole crystalline phase. The diffraction pattern of the basalt fibre indicated partial crystallisation in the amorphous fibre. The main diffraction peaks could be attributed to some form of CaMg-Al-silicate with the possible incorporation of small amounts of iron. The exact composition could not be determined. However, the involved phase appears to be a
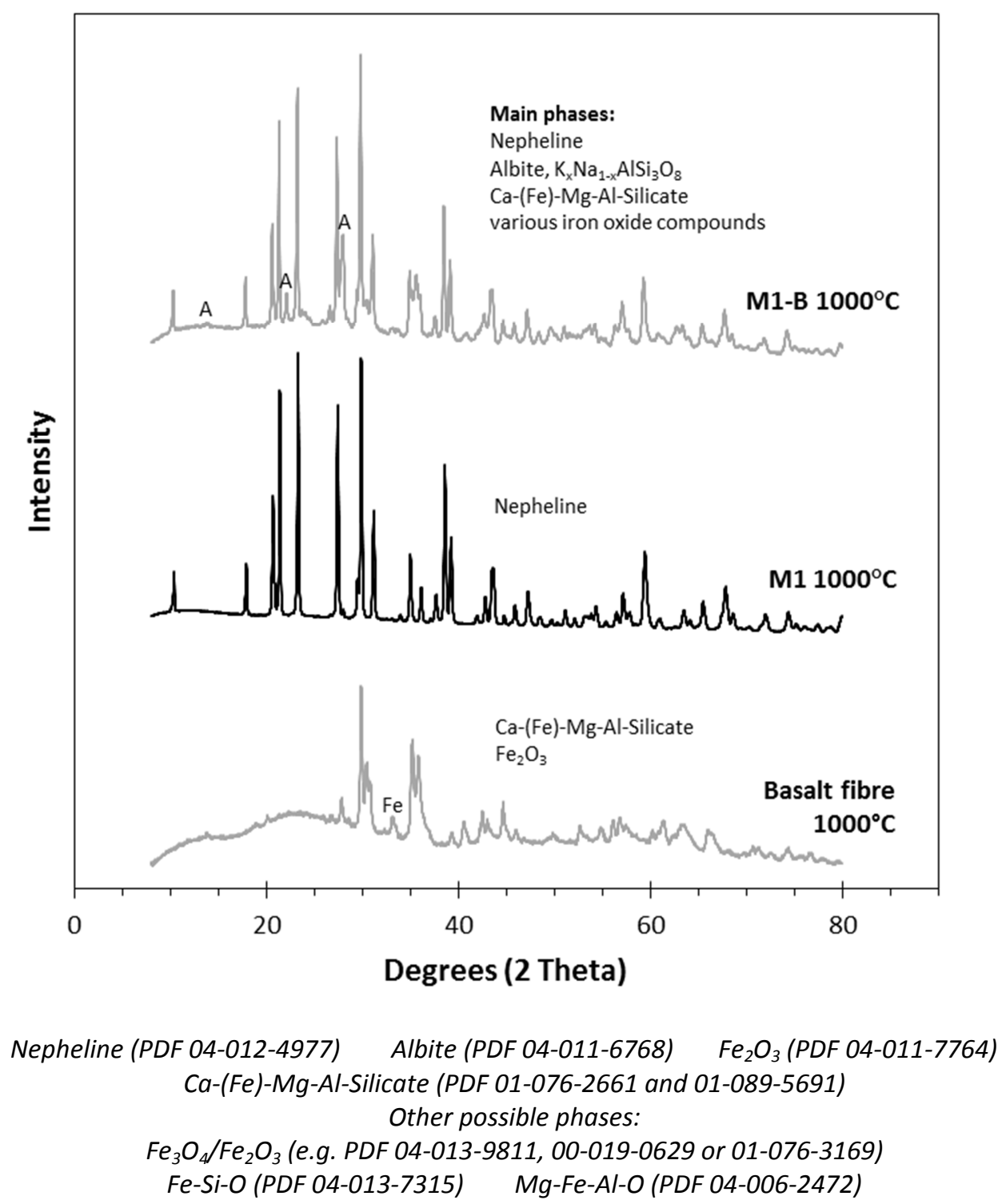

Figure 6.16: Comparison of $\mathrm{x}$-ray diffraction patterns of basalt fibre, M1 matrix and M1-Basalt composite heated to $1000^{\circ} \mathrm{C}$ for $1 \mathrm{~h}$. Note: Due to its relative weakness, only the main Fe peak is labelled in the diffractogram of the heated basalt fibre. All other peaks can be attributed to the $\mathrm{Ca}$ (Fe)-Mg-Al-silicate phase and underlying weaker peaks of the iron phase. All peaks in the diffractogram of the heated matrix belong to the nepheline phase. All these phases are also present in the composite. The only new and clearly identifiable phase in the basalt composite was albite. For reasons of clarity, only the main peaks of the albite phase are labelled 
pyroxene-type solid solution of the respective elements. A form of $\mathrm{Fe}_{2} \mathrm{O}_{3}$ was also detected. The diffraction pattern of the basalt fibre composite was dominated by the nepheline peaks. The number and intensity of these peaks made it somewhat difficult to clearly identify additional phases. However, a good match was found for the previously detected $\mathrm{Ca}-(\mathrm{Fe})-\mathrm{Mg}$-Al-silicate phase and albite $\left(\mathrm{K}_{\mathrm{x}} \mathrm{Na}_{1-\mathrm{x}} \mathrm{AlSi}_{3} \mathrm{O}_{8}\right)$, a new phase that was not detected in either of the two components heated separately. The particular reference pattern for the $\mathrm{Fe}_{2} \mathrm{O}_{3}$ phase found for the heated basalt fibre alone could not be matched to the diffraction pattern of the composite. However, other reference patterns of $\mathrm{Fe}_{2} \mathrm{O}_{3}$ showed a potential fit. Various other iron containing compounds also showed possible matches, including $\mathrm{Fe}_{3} \mathrm{O}_{4}$ as well as a Mg-Fe-Al-O spinel-type phase. Although it seems likely that other crystalline phases may be present in the composite heated to $1000^{\circ} \mathrm{C}$, their formation could not be determined with any certainty.

\subsubsection{Carbon fibre composites}

The heating of the carbon fibre composites to $600^{\circ} \mathrm{C}$ for $1 \mathrm{~h}$ had a drastic effect on the strength and integrity of the samples. The initial visual inspection of the heated samples did not reveal any noticeable cracks but showed an obvious colour change from a marbled black to a dirty white and grey for samples made from desized and sized fibres, respectively. Unexpectedly however, the sized carbon fibre sample turned out to be extremely soft and crumbled like ash upon touching. The desized carbon fibre sample showed somewhat better integrity and although it was still very soft and weak it did not crumble to the same degree as did the sized carbon sample. This extreme decrease in strength and integrity of the carbon fibre samples after heat treatment at $600^{\circ} \mathrm{C}$ was surprising and cannot be explained by the increasing burn out of the carbon fibres alone that was clearly evident from the colour change and inspection of the fracture surface. A repetition of the experiment confirmed the previous observations. The fracture surface revealed that some amount of carbon fibres was still present near the bottom of the samples. Photographs of the colour appearance at the top and bottom surface of the fractured sized and desized fibre samples are shown in Figure 6.17. Despite the slightly different behaviour in their fracture behaviour as well as the colour appearance no clear difference between the 
sized and desized carbon fibre samples could be detected by SEM analysis. Figure 6.18a) shows exemplarily a fractured piece of the sized carbon fibre sample. The presence of carbon fibres at the bottom of the composite bar and increasing burn out of the fibres towards the top surface is displayed in the low magnification image of the fracture surface of the desized carbon fibre sample in Figure 6.18b). However, it is evident from Figure 6.18a) and c) that the remaining carbon fibres are severely degraded, showing a diameter of barely two micrometres. The different microstructural appearance of the matrix in the fibre channels as shown in Figure $6.18 \mathrm{c}$ ) and d) was observed in both samples.

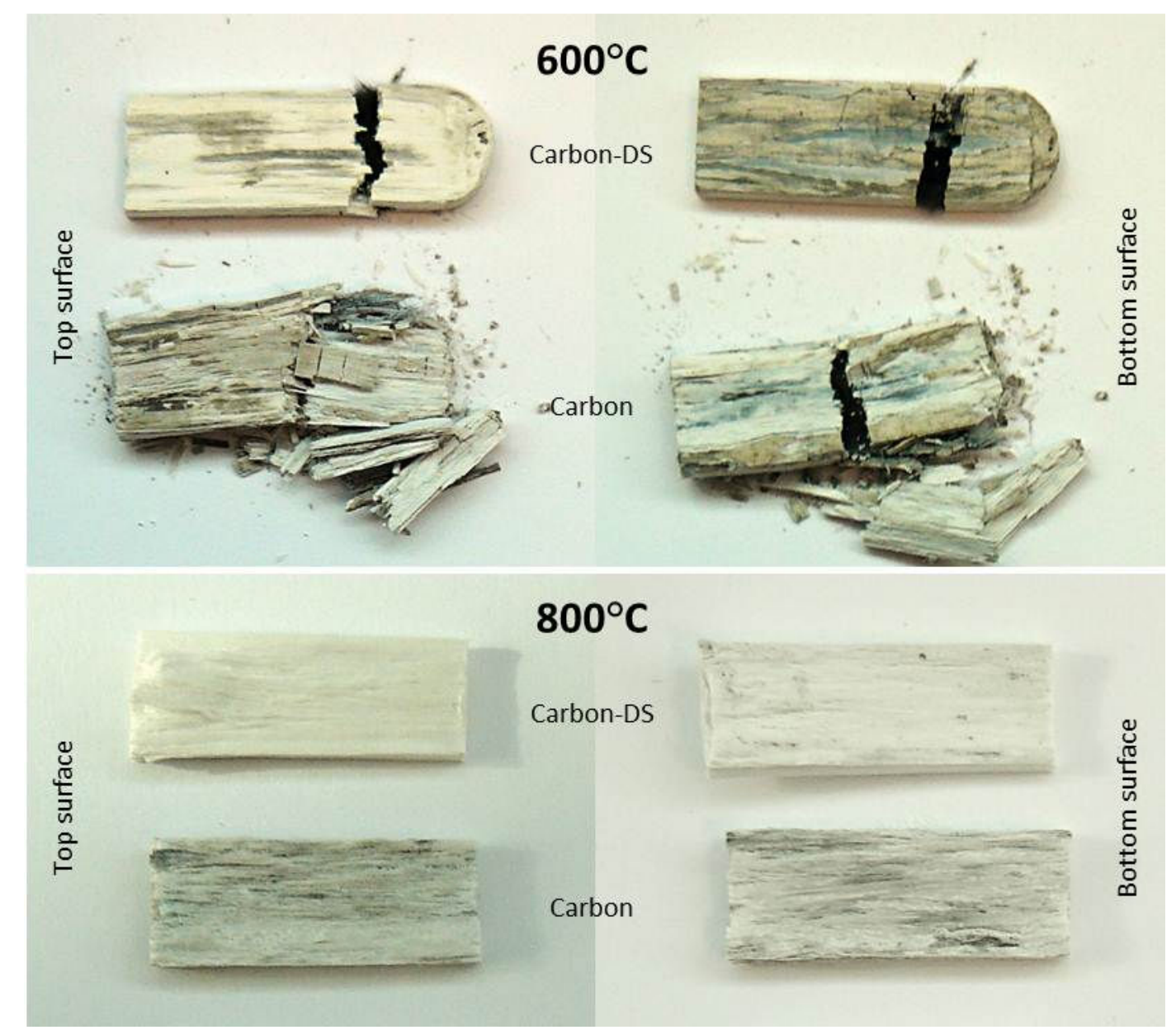

Figure 6.17: Photographs of M1-C and M1-C-DS composite samples heated to 600 and $800^{\circ} \mathrm{C}$

The heating of the carbon fibre composite samples to $800^{\circ} \mathrm{C}$ resulted in the complete burn out of the fibres leaving a porous matrix structure of mostly white colour, see Figure 6.17. The slight discolouration of the sized fibre sample on the top and bottom 
surface, however, suggests the presence of some carbon residue in this sample. In the absence of any fibres, a further analysis of these samples shall be disregarded here. However, it should be mentioned that the $800^{\circ} \mathrm{C}$ samples showed, although still being very weak, increasing strength and better integrity than the $600^{\circ} \mathrm{C}$ samples.
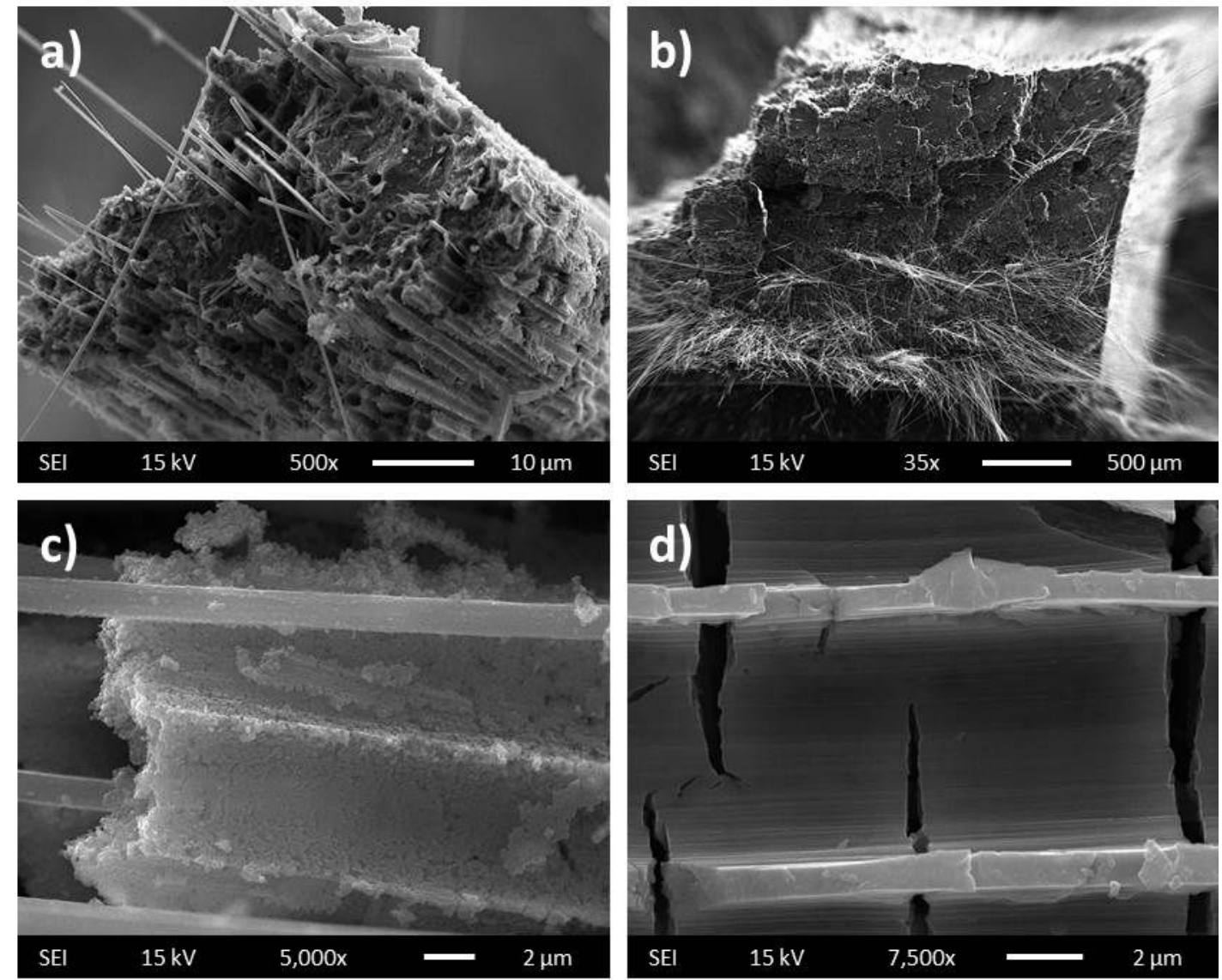

Figure 6.18: SEM micrographs of carbon fibre composite samples heated to $600^{\circ} \mathrm{C}$ : a) fracture piece of sample M1-C, b) fracture surface of sample M1-C-DS, c) and d) showing different microstructural appearances of the matrix in fibre channels and fibre degradation (c) representative for both samples

\subsubsection{Alumina fibre composites}

Visual inspection of the alumina fibre composites after heating to $600^{\circ} \mathrm{C}$ showed a light grey colour on the top surface for the M1-N610 sample made with sized fibres. The bottom surface showed large grey-brown coloured stains resulting from the incomplete burn out of the fibre sizing. The M1-N610 sample appeared largely white after heating to $800^{\circ} \mathrm{C}$ but some discoloured areas remained on the bottom surface. The $1000^{\circ} \mathrm{C}$ sample appeared all white. Essentially no discolouration was observed 
for the desized fibre composite sample M1-N610-DS over the whole temperature range. The strength of the heated composite samples remained high and appeared to increase after heat treatment at 800 and especially $1000^{\circ} \mathrm{C}$.

The SEM analysis revealed no microstructural change between composite samples heated to 600 and $800^{\circ} \mathrm{C}$. The microstructural appearance of the heated samples also showed little difference compared to the room temperature samples. Most fibres, in particular the desized fibres, still appeared to be covered to various extents by a matrix layer whereas other fibres showed largely clean surfaces. The typical appearance of the respective fibres on the fracture surface of the heated composite samples is shown in Figure 6.19.
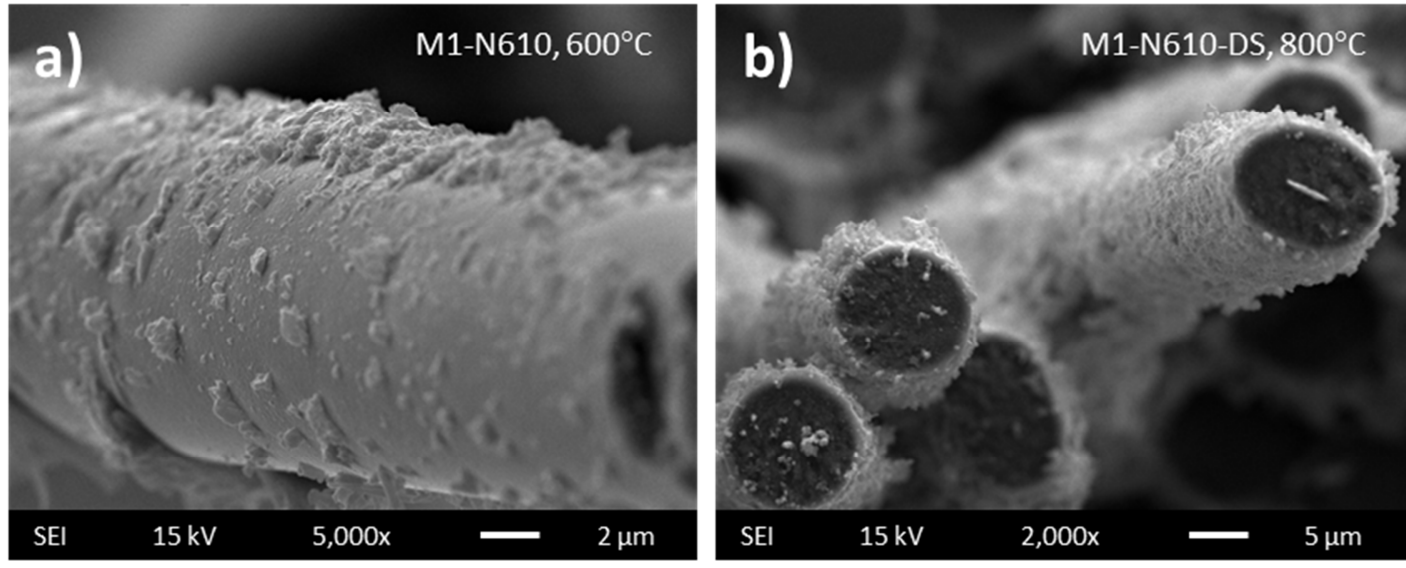

Figure 6.19: Typical appearance of fibres on the fracture surface of a) M1-N610 and b) M1-N610-DS composite samples after heating to 600 and $800^{\circ} \mathrm{C}$. No change in the microstructural appearance of the composite samples was observed between the two temperatures

Fracture surfaces along the fibre orientation revealed a somewhat different appearance of the bonding between the matrix and the fibres. Most noticeable was the lack of the roughness of the matrix layer covering the fibres as observed before (Figure 6.19). No significant difference was observed between the sized and desized fibre composites and the 600 and $800^{\circ} \mathrm{C}$ samples. However, the microstructural appearance of the fibres varied significantly within the same sample. Some fibres appeared largely clean with a smooth surface, other fibres showed a range of thin, thick, relatively dense or porous layers of matrix around the fibre. To indicate the different microstructural appearances, Figure 6.20 presents a selection of SEM 
images that, in one way or another, are representative for composite samples made with sized and desized alumina fibres and heated to 600 or $800^{\circ} \mathrm{C}$.
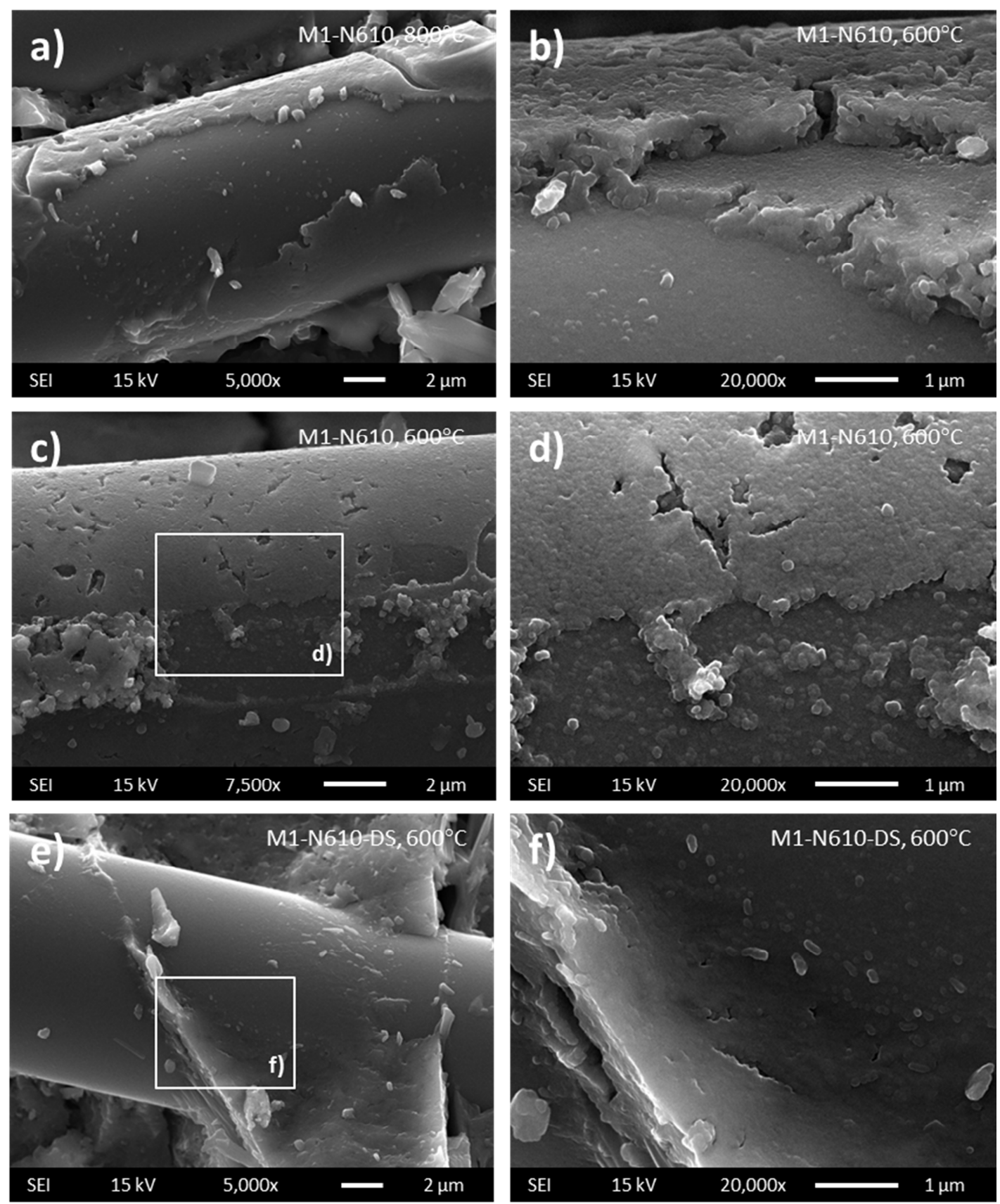

Figure 6.20: Examples of different microstructural appearances of fracture surfaces along the fibre orientation representative for both M1-N610 and M1-N610-DS composites after heating to 600 and $800^{\circ} \mathrm{C}$. No change in the microstructure was observed between 600 and $800^{\circ} \mathrm{C}$

Heating to $1000^{\circ} \mathrm{C}$ resulted in the expected crystallisation and the sintering of the matrix. However, no significant interaction at the fibre-matrix interface was 
observed. Figure 6.21 shows the cross section of some pulled out fibres on the fracture surface of the heated M1-N610-DS composite sample. The appearance of the matrix layer adhering to the fibre surface changed noticeably compared to the
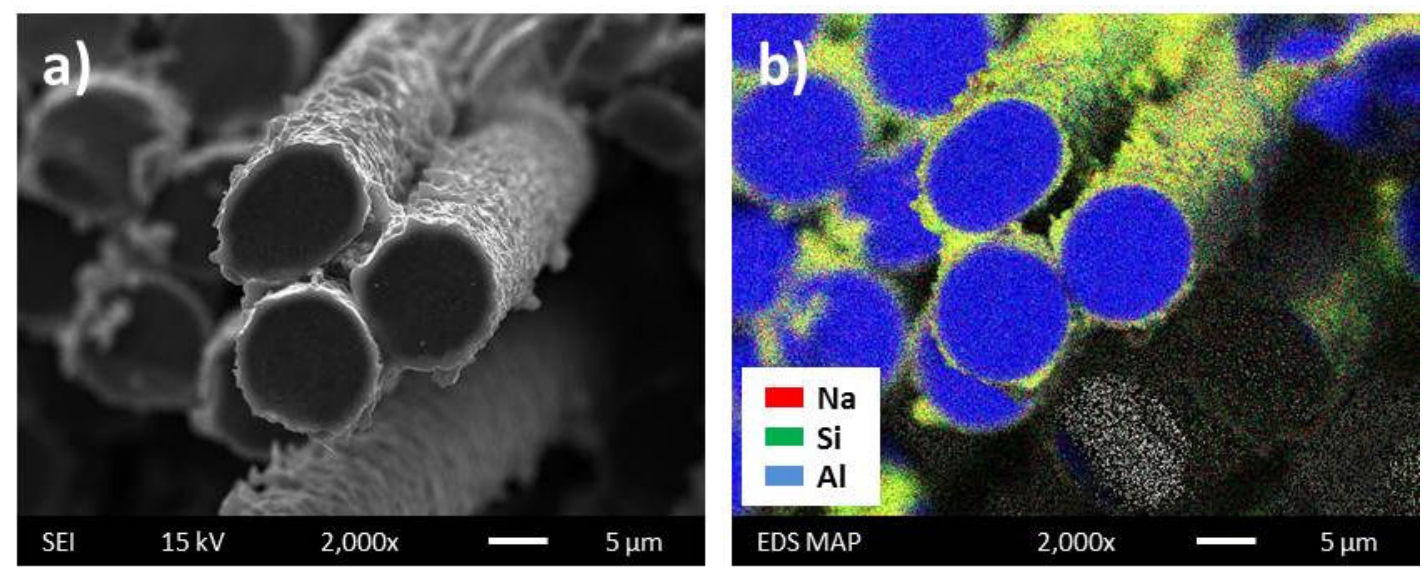

Figure 6.21: SEM micrographs (a) and EDS elemental map (b) of fibres on the fractured surface of M1-N610-DS after heating to $1000^{\circ} \mathrm{C}$
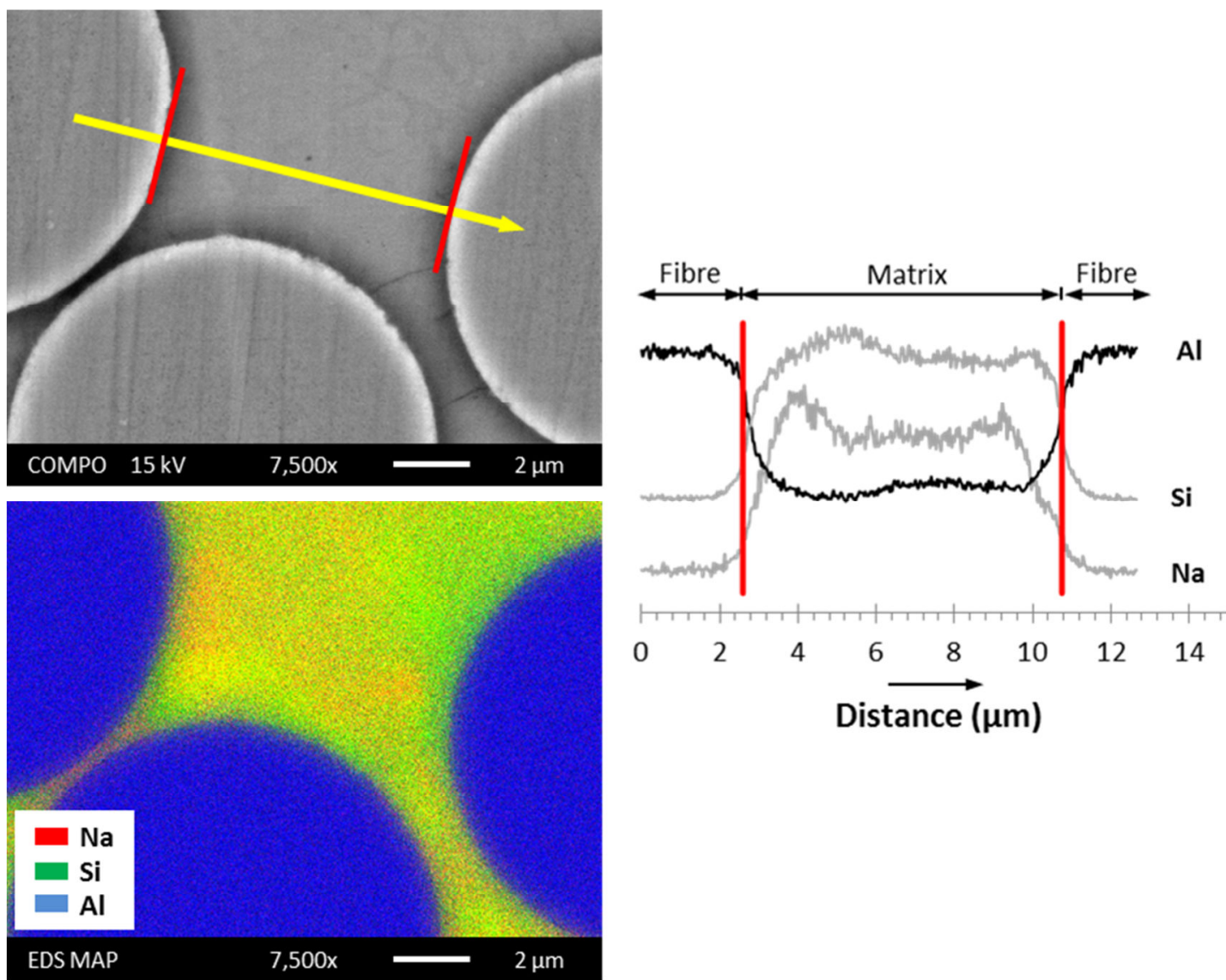

Figure 6.22: Backscattered SEM image with corresponding EDS elemental map and line scan profile across the fibre-matrix interface of the polished surface of the M1-N610 composite heated to $1000^{\circ} \mathrm{C}$ 
composite samples heated to $800^{\circ} \mathrm{C}$. The matrix layer appeared to have densified significantly and sintered onto the fibre surface forming a very rough and strongly bonded surface layer. The EDS map in Figure 6.21(b), however, revealed a very distinct boundary between the alumina fibre and the adhering matrix layer, not indicating any significant interfacial reaction. Further EDS analysis on polished surfaces of the same samples supported this observation, see Figure 6.22. Similar to Figure 6.21(b), the EDS map in Figure 6.22 shows a very distinct boundary between the fibre edge and the surrounding matrix. This is largely consistent with the EDS line scan profile in Figure 6.22 which showed an abrupt change of the concentration of aluminium and silicon across the fibre-matrix interface. The sodium concentration in the matrix, however, appeared to decrease in close proximity to the fibre.

\subsubsection{Discussion}

One of the main incentives for using geopolymer matrix composites over common organic polymer-based composites is their potential application at elevated temperatures, ideally up to $600-800^{\circ} \mathrm{C}$. Taking this into account, the microstructural evolution of the basalt, carbon and alumina fibre composites was investigated at temperatures up to $1000^{\circ} \mathrm{C}$ in order to evaluate the general behaviour and applicability of these three fibre types in geopolymer matrix composites at higher temperature. The microstructural features of the respective samples were described in detail in the previous section. However, as mentioned in the beginning, the model character of these composite samples and especially of the M1 geopolymer matrix shall be emphasised again. While the general behaviour and interactions of the different fibres with the geopolymer matrix observed in this study should be largely transferable to other matrix compositions, the overall performance of the composite is strongly dependent on the exact matrix composition used. Therefore, the following section mainly focuses on a few general aspects of the interfacial interaction between the different fibre types and the matrix and the potential consequences that result from these observations for the use of geopolymer matrix composites at higher temperatures. 
The largely unchanged macro- and microscopic appearance and behaviour of the basalt composites after heating to $600^{\circ} \mathrm{C}$ indicates that the basalt fibres are generally suitable as reinforcements for geopolymers for applications up to this temperature. However, mechanical testing of the heated composites, i.e. residual strength tests, as well as high temperature strength testing is necessary to fully evaluate the suitability of basalt fibres at this temperature. At higher temperatures, the basalt fibres show crystallisation and reaction with the matrix which causes embrittlement of both fibres and composite. Although the crystallisation reactions seem to be mostly inherent to the fibre itself, the presence and chemical nature of the geopolymer matrix clearly influences the degree of crystallisation and the phase formation. After heating to $800^{\circ} \mathrm{C}$, an approximately $2 \mu \mathrm{m}$ wide reaction zone, characterised by the formation of nano-crystallites within this zone, was observed around the inner fibre edges, as was shown in Figure 6.10. The EDS analysis indicated an increased iron concentration within this reaction zone suggesting that the iron present in the basalt fibre starts to increasingly diffuse towards the fibre edges at temperatures between 600 and $800^{\circ} \mathrm{C}$. Based on the results obtained from the XRD analysis, the crystallites that form within this reaction zone can be identified as iron oxide $\left(\mathrm{Fe}_{3} \mathrm{O}_{4}\right)$ as this was the only crystalline phase detected in the heated composite sample other than cristobalite and quartz which are both crystalline impurities from the original halloysite and nepheline which is a crystallisation product of the matrix. The heating to $1000^{\circ} \mathrm{C}$ results in further crystallisation reactions and the formation of at least three distinct reaction zones within the basalt fibres, as shown in Figure 6.12 and Figure 6.14. Whereas the appearance of the two outermost reaction zones appeared to be relatively consistent, the size and shape of crystallites forming in the fibre centre varied significantly between the different fibres. The distribution of the element concentrations within the different reaction zones also varied across the composite sample. However, the reasons for these observations are somewhat unclear. Possible explanations may be slight variations in the chemical composition of the individual basalt fibres or a pronounced influence of the immediate chemical environment around each fibre. That is, fibres in high fibre density regions may show a somewhat different crystallisation and reaction behaviour than fibres that are surrounded by larger amounts of matrix. Nevertheless, based on the SEM and EDS analysis, the 
occurrence of at least three different reaction processes in the basalt fibre composites after heating to $1000^{\circ} \mathrm{C}$ can be established with relative certainty:

(i) The iron present in the basalt fibres concentrates around the inner fibre edges in the two outer reaction zones

(ii) Significant amounts of calcium diffuse from the fibre into the matrix

(iii) Sodium diffuses from the matrix into the fibre

The question which phase crystallises in which reaction zone cannot be answered with any certainty by the SEM and EDS analysis. XRD analysis only revealed two definite crystalline phases in the heated composite other than nepheline which is a product of the matrix crystallisation, i.e. albite and a $\mathrm{Ca}-(\mathrm{Fe})-\mathrm{Mg}$-Al-Silicate phase of unspecified composition. The presence of other crystalline phases, however, in particular various iron oxide compounds and/or a Mg-Fe-Al-O spinel-type phase seems likely but their detection may be concealed by the high intensity of the peaks of the dominant phases. The $\mathrm{Ca}-(\mathrm{Fe})-\mathrm{Mg}-\mathrm{Al}-\mathrm{Silicate}$ as well as an iron oxide phase are also found in the heated fibre alone and are believed to form in the two outer reaction zones. The crystallisation of iron oxide in the fibres and the oxidation of $\mathrm{Fe}_{3} \mathrm{O}_{4}$, found in the $800^{\circ} \mathrm{C}$ sample, to $\mathrm{Fe}_{2} \mathrm{O}_{3}$ after heating to $1000^{\circ} \mathrm{C}$ would also explain the observed colour change in the fibres and composite, respectively.

The role of calcium in the matrix is not fully understood as it does not appear to influence the crystallisation of the matrix into nepheline. The XRD analysis does not indicate the formation of a Ca-containing crystalline phase in the matrix since the detected $\mathrm{Ca}-(\mathrm{Fe})-\mathrm{Mg}-\mathrm{Al}-\mathrm{Silicate}$ phase is believed to crystallise within the outer fibre region. However, the EDS elemental maps in Figure 6.14 suggest that some $\mathrm{Ca}, \mathrm{Mg}$ and Si-rich crystals may form in the matrix but the amount of this phace may be too small to be detected by XRD.

The formation of albite is only observed in the composite and is therefore clearly a product of the interaction between the fibres and the matrix caused by the interdiffusion of the alkali atoms. The crystallisation of the albite phase is expected 
to occur most likely in the second and/or third reaction zone within the fibre. However, the diffusion of the sodium atoms may play a larger role in the overall reaction behaviour of the fibre and the composite, respectively, than simply causing the formation of albite. As mentioned before, comparison of the crystallisation behaviour of the basalt fibres alone and the composite sample after heating to $1000^{\circ} \mathrm{C}$ clearly shows that the presence and chemical nature of the matrix has significant effect on the degree of crystallisation and the phase formation. While the heating of the basalt fibres alone to $1000^{\circ} \mathrm{C}$ indicated some degree of sintering and softening of the fibres, the presence of the highly alkaline matrix seems to amplify these processes considerably. By diffusing into the fibres, the sodium is believed to act like a flux, causing a significant decrease of the melting temperature and at least partial melting of the basalt fibre due the formation of eutectic mixtures. The lower viscosity facilitates easier diffusion transport and reinforces the crystallisation reactions that occur in the fibre. The accumulation of small pores along the fibre edges in the $800^{\circ} \mathrm{C}$ sample (see Figure 6.10) and their subsequent transport into the fibre, resulting in the formation of large pores within the fibres (see Figure 6.11), already suggests the early softening of the basalt fibre. Due to the reduced melting/softening point, the basalt fibres essentially act like a glassy phase during the sintering process. Therefore, the use of basalt fibres has to be considered generally unsuitable for applications exceeding $600^{\circ} \mathrm{C}$.

The carbon fibre composites showed significant degradation and fibre burnout after heating in air to $600^{\circ} \mathrm{C}$. Due to the relatively small sample size and the direct exposure of the fibres to the oven atmosphere on the cut end surfaces of the sample, the fibre burnout may have been more severe than in the case of a larger sample. However, experiments with small amounts of carbon fibres fully enclosed in a thick geopolymer layer also showed significant fibre degradation under the same conditions. Besides the apparent fibre burnout, a dramatic effect on the strength and integrity of the matrix was observed. Although the residual strength of the composite after heating to $600^{\circ} \mathrm{C}$ would be expected to be very weak due to a weakening of the matrix itself and the very porous structure caused by the burnout of the incorporated carbon fibres, the ash-like crumbling of the heated composites upon touching was not 
expected. Therefore, the oxidation of the carbon fibres is believed to have some additional effect resulting in the disintegration of the heated composite sample. It is also evident from the results that the fibre sizing unfavourably affects the sample integrity. However, similar behaviour was not observed for the sized basalt and alumina fibre composite samples. Therefore, the effect of the fibre sizing on the composite behaviour at elevated temperatures appears to be dependent on the particular nature of the fibre sizings. In that regard, the use of desized fibres in geopolymer composites may be favourable in some instances when applied to higher temperatures. After heating to $800^{\circ} \mathrm{C}$, all carbon fibres had burned out. However, the samples showed better strength and structural integrity than after heating to $600^{\circ} \mathrm{C}$ which can be attributed to the onset of matrix sintering. Thus, the temperature region around $600^{\circ} \mathrm{C}$ seems to be critical. A strength minimum at $600^{\circ} \mathrm{C}$ was also observed by Foden [9] for $3 \mathrm{~mm}$ thick carbon composite specimens, see Figure 2.5. However, much better strength retention was reported. This was explained by the protective effect of the geopolymer matrix on the burnout of the carbon fibres. Although the high temperature behaviour of the composite is strongly dependent on the properties and composition of the particular geopolymer matrix, the general ability of any aluminosilicate geopolymer matrix to protect carbon fibres from oxidation seems arguable. It is agreed that carbon fibre reinforced geopolymer matrix composites can withstand short-term exposure to high temperature and direct fire exposure, simulated by the use of a Bunsen burner, without causing immediate failure, burning or smoke development. Nevertheless, while the geopolymer matrix may be able to retard the fibre burnout to some degree, any long-term protection is not evident. Therefore, the safe long-term application of carbon fibre geopolymer composites is restricted to temperatures far below $600^{\circ} \mathrm{C}$.

Of the three main fibre types investigated, alumina are the only fibres that are principally suitable for applications of geopolymer composites at temperatures above $600^{\circ} \mathrm{C}$. Similar to the geopolymer binder at room temperature, the alumina fibres appeared to be wetted well by the softening matrix at higher temperatures which is evident by the matrix layer covering large parts of the fibre surfaces on the fractured composite samples (Figure 6.19 to Figure 6.21). In combination with the sintering 
and densification of the matrix, a significant increase of the interfacial strength is assumed. Based on the examination of the fractured surfaces of the composite samples heated to $1000^{\circ} \mathrm{C}$, the matrix appeared to have sintered strongly with the fibres forming a dense matrix layer around the fibres. However, further SEM and EDS analysis could not confirm any apparent interfacial reaction with the formation of a distinct interphase region between the two constituents. Some of the micrographs in Figure 6.20 suggest the formation of a layered matrix structure in the direct vicinity of the fibres with slight differences in the chemical composition and microstructure of these layers. The EDS line scan profile in Figure 6.22 suggests that the sodium content decreases in close proximity to the alumina fibres compared to matrix-rich areas of the composites. This potential change in the sodium concentration close to the fibre surface could explain the formation of apparent matrix layers of slightly different chemical composition and microstructure around the fibres. However, these observations only give a general indication of the behaviour of Nextel 610 alumina fibres in a geopolymer matrix and closer investigation of the interaction with a more suitable matrix composition coupled with mechanical measurements are necessary if such high temperature exposures are considered.

The temperature region around $800-1000^{\circ} \mathrm{C}$ essentially marks the extreme limit of geopolymer matrix composites beyond which the geopolymer matrix gradually transitions into a crystalline ceramic. In that regard, the use of geopolymer matrix composites as precursors for the fabrication of ceramic matrix composites presents an interesting possibility. Especially the fabrication of complex shaped ceramic composites from the geopolymer precursor may have considerable potential. Depending on the type of alkali cation used in the activation of the geopolymer binder, nepheline, leucite or other crystalline matrix phases may be formed. The idea of geopolymer matrix composites as precursors for ceramics is considered in some more detail in the following chapter. 


\section{Chapter 7}

\section{Geopolymer composites as precursors for CMCs - General considerations and initial results}

As part of a small side project, the general idea of geopolymer composites as precursors for ceramic composites was considered. The possibility of crystallising the geopolymer matrix into nepheline and nepheline-matrix composites, respectively, was already shown in the previous chapters. Here, particular interest was taken in the principal feasibility of replacing the $\mathrm{Na}^{+}$cations in the geopolymer matrix with $\mathrm{NH}_{4}{ }^{+}$ ions via ion exchange in order to transform the geopolymer matrix into more temperature stable mullite. For the purpose of the present work, the results will be limited to some basic observations and XRD phase analysis of the fired samples.

\subsection{1 lon exchange experiments}

Fractured unreinforced M1 bar and cut M1-N610 composite pieces, both of about $30-40 \mathrm{~mm}$ length, were used as samples for the ion exchange experiments. A powdered unreinforced geopolymer sample was also tested for comparison. Two samples of each sample type were ion exchanged according to the process described in chapter 3.6 and subsequently fired at 1000 and $1100^{\circ} \mathrm{C}$, respectively. 
No obvious cracking was observed for the bulk samples during the ion exchange process. However, one of the unreinforced bar samples cracked during the subsequent drying process. Firing of the unreinforced bar samples at both temperatures resulted in large gaping cracks on the surface as shown in Figure 7.1(a). Inspection of the cross-section of the bar samples revealed a porous core of glassy appearance surrounded by an approximately one millimetre wide, much denser outer zone, see Figure 7.1(b). The macroscopic appearance of the bar samples was confirmed by the SEM analysis as is evident from Figure 7.2(a). The outer area was found to be very dense even at higher magnification.
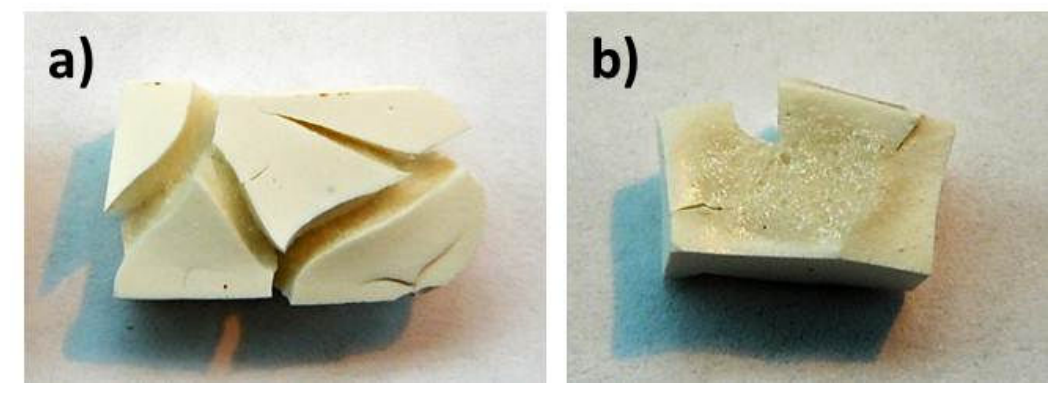

Figure 7.1: Typical appearance of ion exchanged bar specimens after firing at 1000 and $1100^{\circ} \mathrm{C}$

The visual appearance of the composite samples fired at 1000 and $1100^{\circ} \mathrm{C}$ was also similar and no apparent differences to the macroscopic appearance of the untreated composite samples after heating to $1000^{\circ} \mathrm{C}$ could be observed. The SEM analysis revealed a largely heterogeneous, porous structure (Figure 7.2(c)). Low magnification micrographs of polished cross-sections of both unreinforced and composite samples were analysed by EDS mapping for sodium to visualise the extent of the ion exchange in the two bulk sample types, as shown in Figure 7.2. A decrease of the sodium concentration from the centre towards the surface of the samples is clearly evident from the colour gradient in the EDS maps indicating the successful ion exchange in the outer area of the bulk samples. The effective penetration depth is about $0.5-1 \mathrm{~mm}$ from the surface.

The powder samples showed some degree of inter-particle sintering after firing at $1100^{\circ} \mathrm{C}$. However, the interconnectivity between individual particles was relatively 
weak and the sample could be ground easily for the subsequent XRD analysis. The powdered sampled were not examined under the SEM.
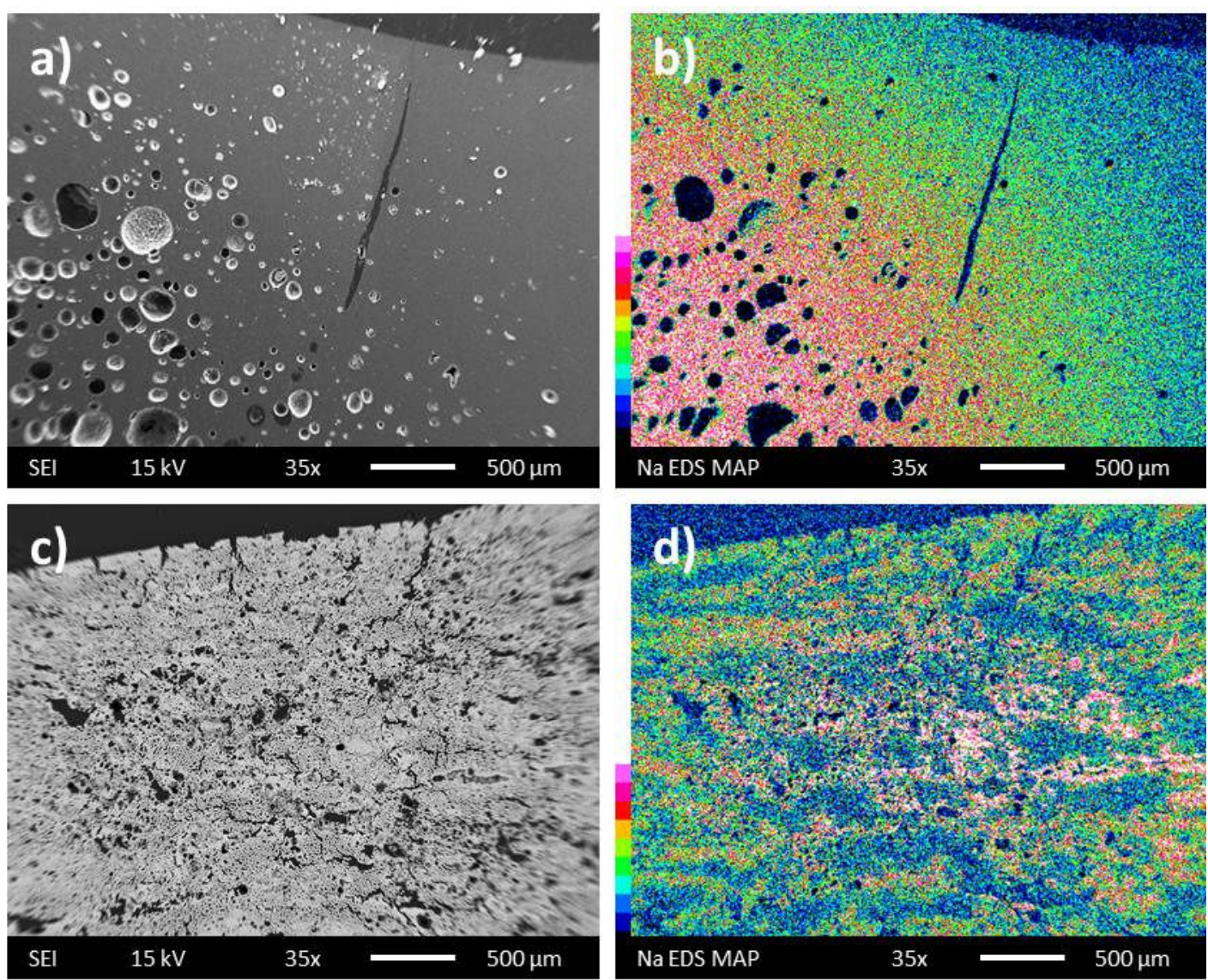

Figure 7.2: EDS maps on polished surfaces showing sodium distribution in ion exchanged unreinforced bar $(\mathrm{a}, \mathrm{b})$ and Nextel 610 fibre reinforced composite (c, d) specimens with M1 matrix after heating to $1000^{\circ} \mathrm{C}(\mathrm{Na}$ concentration increases from black to white colour as indicated by sidebar)

The x-ray diffraction patterns of the three different sample types after heating to 1000 and $1100^{\circ} \mathrm{C}$ are shown in Figure 7.3. The slight background elevation found in all diffractograms indicates that all samples remained at least partially amorphous. Also, quartz and cristobalite impurities were found to various degrees in all samples. Their amount generally decreased with increasing temperature. Conversely, the amount of mullite, which was also detected in all samples, generally increased with increasing temperature. The formation of mullite in heated samples was previously not observed and is a result of the ion exchange. Traces of nepheline, the main phase of the untreated, heated geopolymer matrix, were only found in the composite 
sample after heating to $1000^{\circ} \mathrm{C}$. The dominant alumina peaks in the diffractograms of the composite samples can be attributed to the crystalline fibres.

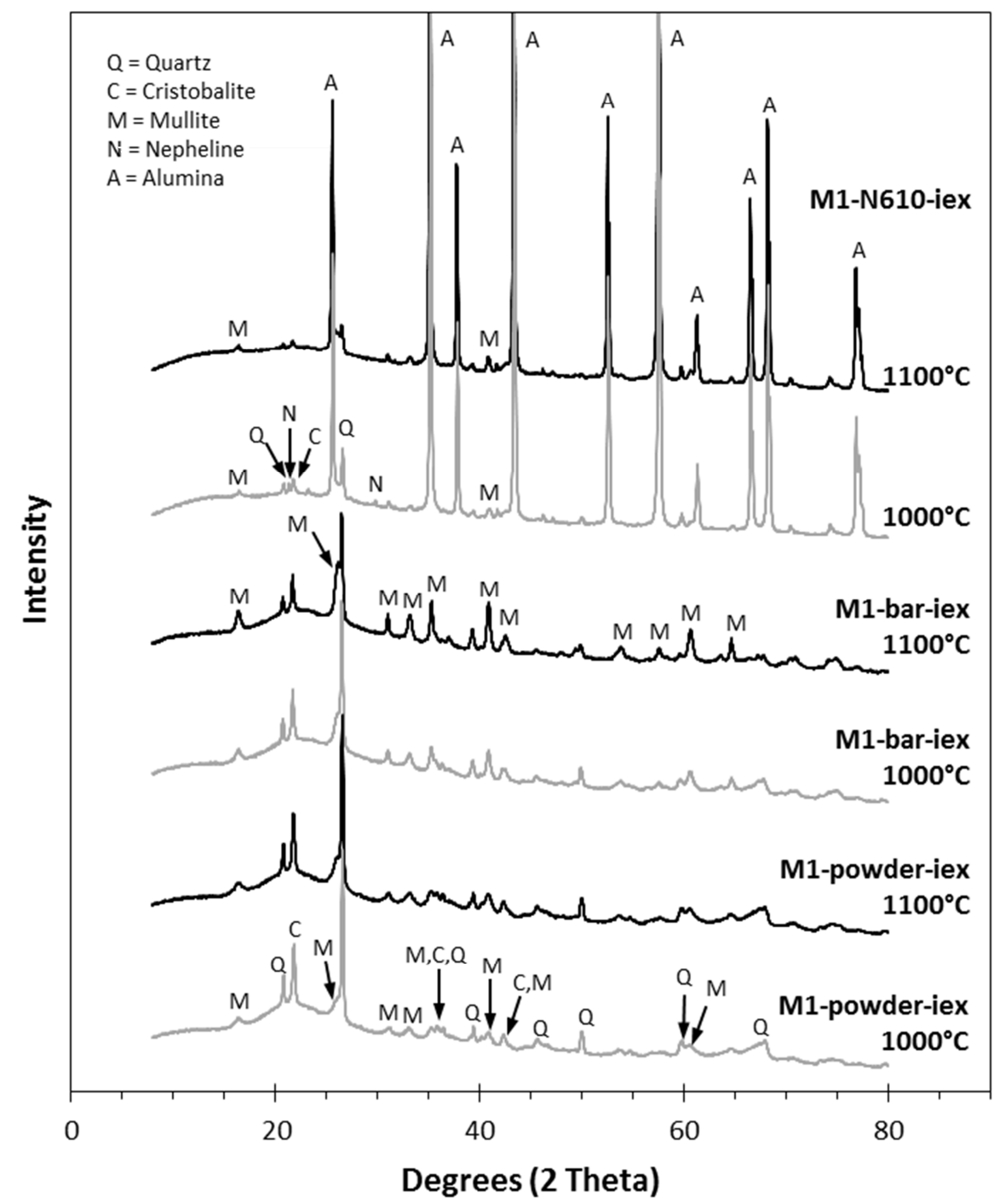

Quartz (PDF 01-089-8935)

Cristobalite (PDF 04-007-2134)

Mullite (PDF 04-009-3667)

Nepheline (PDF 04-012-4977)

Alumina (PDF 01-076-7775)

Figure 7.3: Comparison of x-ray diffraction patterns of ion exchanged M1 powder samples, bar pieces and M1-N610 composite samples after firing at 1000 and $1100^{\circ} \mathrm{C}$

\subsubsection{Discussion}

The results clearly show the successful ion exchange in the outer regions of the bulk geopolymer bars and composites as well as the formation of some amount of mullite. 
The formation of nepheline, on the other hand, was mostly suppressed. Therefore, the principal idea behind these experiments can be considered successful. However, there appear to be some limitations that have to be considered. As shown in Figure 7.2, the maximum penetration depth up to which the ion exchange of the sodium ions in the bulk samples was effective was limited to around one millimetre. Improvements in that regard may be possible by altering several parameters of the ion exchange process, namely the process time and the concentration of the ammonium solution, and by increasing the matrix porosity. Increasing the initial matrix porosity and a uniform ion exchange across the whole sample may also help to promote a more homogeneous densification of the matrix. In particular for the unreinforced bar samples, a very high degree of densification in the outer ion exchanged sample areas could be observed. The densification of the outer areas prevents the escape of entrapped air in the pores in the inner region of the sample, leading to the expansion of these pores and ultimately to the formation of the large open cracks on the sample surface.

Another aspect that has to be considered is the composition of the geopolymer matrix. For the present experiments, existing samples made with the M1 geopolymer composition were used. The chemical composition of the M1 matrix, however, is very different from the nominal composition of mullite $\left(3 \mathrm{Al}_{2} \mathrm{O}_{3} \cdot 2 \mathrm{SiO}_{2}\right)$. Therefore, significant improvement in terms of mullite formation would be expected from an optimised geopolymer matrix composition. However, the idea of geopolymer matrix composites as precursors for ceramic composites can be extended even further. Recent research at Victoria University investigated the transformation of geopolymers into silicon-aluminium-oxynitride ceramics, or sialons [104]. Sialons are a type of advanced ceramics characterised by a combination of desirable properties such as high strength up to high temperatures as well as good oxidation, wear and, especially, corrosion resistance. The direct formation of fibre reinforced sialon-matrix composites from a geopolymer precursor would open new possibilities for the fabrication of ceramic composites with considerable commercial potential. However, the subject of geopolymer composite as precursors for ceramic composites 
presents a rather separate topic from the original idea of geopolymer matrix composites and must be addressed in future studies. 


\section{Chapter 8}

\section{Summary and conclusions}

The aim of this study was the general characterisation of the effects of a number of key parameters on the mechanical behaviour of geopolymer matrix composites in order to improve the basic understanding of these materials and to evaluate their general potential as composites materials for mid-temperature applications. For that purpose, the following four main aspects were investigated:

(i) characterisation of a model geopolymer matrix system

(ii) evaluation of the mechanical properties and failure behaviour of unidirectional fibre reinforced geopolymer matrix composites

(iii) analysis of fibre-matrix interactions up to $1000^{\circ} \mathrm{C}$

(iv) initial experiments on geopolymer matrix composites as precursors for CMCs

The main objective in the investigation of the matrix system was the characterisation of the mechanical properties of the matrix. It was shown that the strength of the geopolymer matrix was influenced by various parameters such as the overall matrix composition, the fabrication method and the mixing time. The main matrix composition used in this work (M1) achieved an average compressive strength of $\sim 79 \mathrm{MPa}$ and an average flexural strength and modulus of $\sim 10 \mathrm{MPa}$ and $8.5 \mathrm{GPa}$, 
respectively, for the best sample batch for each test method. While the distribution of the strength values within any given sample batch was reasonable, significant strength variations were observed between different batches. The strength variation between different sample batches was random and unpredictable. The reasons for this apparent lack of reproducibility were somewhat unclear but are believed to be most likely linked to the fabrication method and the insufficient mixing of the geopolymer binder. It was suggested that the variation of the strength is due to local structural defects in the sample. Although the particular mixing process used in this study may not have been optimal and better results may be achieved with a more advanced high-shear mixing process, this does not explain why the properties of different sample batches of the same material, prepared according to the same process should vary to the observed extent. Comparison between the present results and similar compositions in the literature revealed that many of the reported strength values are in fact similar to those measured for the defective samples here. Therefore, it is suggested that the strength values of metakaolin-based geopolymers are widely underrepresented. Furthermore, these observations suggest that the fabrication and consistent reproducibility of high strength geopolymers is much more difficult than commonly assumed. However, some of these problems may be overcome by optimising the geopolymer composition. The optimised composition M5 achieved extremely high strength with a maximum compressive strength of $145 \mathrm{MPa}$. This strength is much higher than previously reported values for similar geopolymer formulations.

The mechanical properties of geopolymer matrix composites were investigated on unidirectional fibre reinforced composites using basalt, carbon and alumina fibres. Other parameters that were evaluated included the fibre sizing, the matrix strength, the span-to-depth ratio and the specimen dimensions. A preliminary corrosion test showed that all three fibre types were chemically stable under highly alkaline conditions and were therefore suitable for the use in geopolymer composites. Significant improvements of the mechanical properties compared to the unreinforced geopolymer matrix were achieved with all fibre types. The mechanical behaviour of the composites is strongly dependent on the fibre properties, in particular the fibre 
modulus. The alumina fibre composites achieved the highest flexural stress with a maximum value of $470 \mathrm{MPa}$ and a fibre content of $\sim 30$ vol.-\%. Both basalt and carbon fibre composites showed maximum flexural strength values around $200 \mathrm{MPa}$. All composite types showed considerable post-fracture strength. However, only the basalt composites showed tensile failure mode. The carbon and alumina composites generally failed due to interlaminar shear. Different extents of compressive failure were also observed for all composite types. Therefore, the failure behaviour of geopolymer composites under flexural loading is complex. The applicability of the weak matrix composites (WMC) concept to describe the mechanical behaviour of geopolymer matrix composites was discussed. Whereas the WMC concept appeared to be generally applicable to describe the mechanical behaviour of the basalt composites, it may not be able to adequately describe the behaviour of the carbon and alumina composites. This is due to the extreme elastic mismatch between these high-modulus fibres and the weak matrix. That might also be the reason why the matrix strength has a more significant effect on the high modulus carbon fibre composites than on the basalt composites.

The effect of the fibre sizing on the composite properties was less clear. Whereas the desizing of the carbon fibres resulted in an increase of the composite strength, a reverse effect was observed for the alumina composites. Thus, the effect of the fibre sizing depends on the chemical nature of the sizing. However, it was unclear what exactly happens to the fibre sizing in the composite. Most likely the sizing dissolves and gets incorporated into the matrix. In any case, the effect of the fibre sizing on the composite properties was relatively small in comparison to the influence of the fibre type. Unlike previous observations, a significant change in the failure behaviour for desized fibres could not be confirmed. Consequently, the removal of the fibre sizing does not seem necessary for room temperature applications. However, the example of the carbon fibre showed that the fibre sizing can have significant effect on the composite strength and integrity of the matrix at higher temperatures.

The effect of different testing spans and sample dimensions on the composite properties was investigated on basalt fibre composites. Both parameters were shown 
to have great influence on the measured strength and the observed failure mode. Therefore, the standardisation of testing parameters is highly recommended in order to improve comparability between different studies.

One factor that strongly influenced the composite strength was the particular fabrication method. A continuous mixing process was used in order to extend the effective use time of the geopolymer binder and to increase the number of samples that could be produced from one batch of binder. It was shown that the composite strength increased with the order of fabrication within a fabrication series. Whereas this effect was unintended, these observations may offer some valuable information for potential up-scaling processes.

The microstructural investigation of the fibre-matrix interaction revealed no chemical reaction between the fibres and the matrix at room temperature. Analysis of the heated composites indicated that basalt fibre composites may be used up to $600^{\circ} \mathrm{C}$ without significant degradation of the fibre. However, sintering, melting, crystallisation and embrittlement of the fibre occurred at higher temperature. The heating of the carbon fibre composites to $600^{\circ} \mathrm{C}$ had drastic effect on the strength and integrity of the sample, in particular, when using sized carbon fibres. The alumina fibres showed very good wetting and bonding behaviour but otherwise little reaction with the matrix even after heating to $1000^{\circ} \mathrm{C}$.

The ion exchange experiments clearly indicate that it is possible to influence the phase formation at higher temperatures. This topic requires further investigation but the general idea of using geopolymer matrix composites as precursors for ceramic composites may be considered in regards of more cost-efficient fabrication method for ceramic composites.

Geopolymer matrix composites show great potential as fire-resistant composite materials for predominantly near room temperature applications. In particular basalt fibre composites are of great interest due to their comparatively low cost and good mechanical performance. Whereas the microstructural investigations indicate that 
basalt fibres can potentially be used in geopolymer matrices up to $600^{\circ} \mathrm{C}$, the success of the application of geopolymer matrix composites at higher temperatures is seen critically and depends on the development of suitable matrices. For applications at higher temperatures the transformation of geopolymer matrix composites into ceramic matrix composites may be of greater potential. However, this somewhat counteracts the initial advantage of the low temperature and cost-efficient fabrication process of geopolymer composites. 


\section{References}

[1] F. Cardarelli, Composite Materials, in Materials Handbook (2nd ed.), Springer, London (2008), Chapter 18

[2] J. Davidovits, Geopolymer - Chemistry and Applications, Institut Géopolymère, Saint-Quentin (2008)

[3] S.C. Foerster, Alkaliaktivierte Aluminosilikat-Verbundkeramiken, $P h D$ Thesis, ETH Zürich, Switzerland (1994)

[4] S.C. Foerster, T. Graule, L.J. Gauckler, Strength and toughness of reinforced chemically bonded ceramics in: cement technology, edited by EM Gartner and H Uchikawa, Ceramic Transactions 40 (1994), 247-256

[5] S.C. Foerster, T. Graule, L.J. Gauckler, Thermal and mechanical properties of alkali-activated alumino-silicate based, high performance composites in: Advanced structural fiber composites edited by $\mathrm{P}$ Vincenzini, Advances in science and technology 7 (1995), 117-124

[6] R.E. Lyon, U. Sorathia, P. Balaguru, A. Foden, J. Davidovits, M. Davidovics, Fire response of geopolymer structural composites, Proceedings of the $1^{\text {st }}$ International conference on composites in infrastructure ICCI, 1996

[7] R.E. Lyon, P.N. Balaguru, A. Foden, U. Sorathia, J. Davidovits, M. Davidovis, Fire resistant aluminosilicate composites, Fire and Materials 21 (1997), 67-73

[8] A. Foden, R. Lyon, P.N. Balaguru, J. Davidovits, High temperature inorganic resin for use in fibre reinforced composites, Proceedings of the $I^{\text {st }}$ International conference on composites in infrastructure ICCI, 1996 
[9] A.. Foden, Mechanical properties and material characterization of polysialate structural composites, PhD Thesis, Rutgers State University, USA (1999)

[10] J. Hammell, P.N. Balaguru, R. Lyon, Influence of reinforcement types on the flexural properties of geopolymer composites, Proceedings of the $43^{\text {rd }}$ International SAMPE Symposium, 1998

[11] J.A. Hammell, The influence of matrix composition and reinforcement type on the properties of polysialate composites, $P h D$ Thesis, Rutgers State University, USA (2000)

[12] J.A. Hammell, P.N. Balaguru, R.E. Lyon, Strength retention of fire resistant aluminosilicate-carbon composites under wet-dry conditions, Composites: Part B 31 (2000), 107-111

[13] P. He, D. Jia, T. Lin, M. Wang, Y. Zhou, Effects of high-temperature heat treatment on the mechanical properties of unidirectional carbon fibre reinforced geopolymer composites, Ceramics International 36 (2010), $1447-1453$

[14] P. He, D. Jia, M. Wang, Y. Zhou, Improvement of high-temperature mechanical properties of heat treated $\mathrm{C}_{\mathrm{f}}$ /geopolymer composites by Sol$\mathrm{SiO}_{2}$ impregnation, J. Eur. Ceram. Soc. 30 (2010), 3053-3061

[15] P. He, D. Jia, Interface evolution of the $\mathrm{C}_{\mathrm{f}}$ /leucite composites derived from $\mathrm{C}_{\mathrm{f}} /$ geopolymer composites, Ceramics International 39 (2013), 1203-1208

[16] O. Bortnovsky, P. Bezucha, J. Dedecek, Z. Sobalik, V. Vodickova, D. Kroisova, P. Roubicek, M. Urbanova, Properties of phosphorus-containing geopolymer matrix and fibre reinforced composites, in D. Singh, W.M. Kriven, J. Salem (ed.), Mechanical properties and performance of engineering ceramics and composites IV (2009), 283-299

[17] D.H. Tran, D. Kroisova, P. Louda, O. Bortnovsky, P. Bezucha, Effect of curing temperature on the flexural properties of silica-based geopolymer carbon reinforced composites, Journal of Achievments in Materials and Manufacturing Engineering 37 (2009), 492-497

[18] D. Pernica, P.N.B. Reis, J.A.M. Ferreira, P. Louda, Effect of test consitions on the bending strength of a geopolymer-reinforced composite, J. Mater. Sci. 45 (2007), 744-749

[19] D.W. Radford, A. Grabher, J. Bridge, Inorganic polymer matrix composite strength related to interface condition, Materials 2 (2009), 2216-2227 
[20] T. Lin, D. Jia, P. He, M. Wang, D. Liang, Effects of fiber length on mechanical properties and fracture behaviour of short carbon fiber reinforced geopolymer matrix composites, Mater. Sci. Eng. A 497 (2008), $181-185$

[21] T. Lin, D. Jia, M. Wang, P. He, D. Liang, Effects of fibre content on mechanical properties and fracture behaviour of short carbon fibre reinforced geopolymer matrix composites, Bull. Mater. Sci. 32 (2009), 7781

[22] T. Lin, D. Jia, P. He, M. Wang, In situ crack growth observation and fracture behaviour of short carbon fibre reinforced geopolymer matrix composites, Materials Science and Engineering A 527 (2010), 2404-2407

[23] W.M. Kriven, J.B. Bell, M. Gordon, Microstructure and microchemistry of fully-reacted geopolymers and geopolymer matrix composites, in Advances in Ceramic Matrix Composites IX, Ceramic Transactions 153 (2003), 227-250

[24] E. Rill, D.R. Lowry, W.M. Kriven, Properties of basalt fibre reinforced geopolymer composites, Ceramic Engineering and Science Proceedings 31 (2010), 57-67

[25] S.S. Musil, G.P. Kutyla, W.M. Kriven, The effect of basalt chopped fibre reinforcement on the mechanical properties of potassium based geopolymer, Ceramic Engineering and Science Proceedings 33 [10] (2012), 31-42

[26] W. Li, J. Xu, Mechanical properties of basalt fibre reinforced geopolymeric concrete under impact load, Material Science and Engineering A 505 (2009), 178-186.

[27] W. Li, J. Xu, Impact characterization of basalt fibre reinforced geopolymeric concrete using a $100 \mathrm{~mm}$-diameter split Hopkinson pressure bar, Material Science and Engineering A 513-514 (2009), 145-153

[28] D.P. Dias, C. Thaumaturgo, Fracture toughness of geopolymeric concretes reinforced with basalt fibres, Cement and Concrete Composites 27 (2005), $49-54$

[29] D.C. Comrie, W.M. Kriven, Composite cold ceramic geopolymer in a refractory application, Ceramic Transactions 153 (2003), 211-225

[30] C. Defazio, M.D. Arafa, P.N. Balaguru, Functional geopolymer composites for structural ceramic applications, Final Report, CeramRU9163, Center for Advanced Infrastructure and Transportation, Rutgers State University, USA, 2006, Online available from: http://cait.rutgers.edu/files/Ceram-RU9163.pdf (Last accessed: 6.11.2013) 
[31] D. Hull, T.W. Clyne, An introduction to composite materials (2nd ed.), Cambridge University Press (1996)

[32] D. Roylance, Introduction to composite materials, Massachusetts Institute of Technology (2000)

[33] A. Kelly, A. Mortensen, Composite Materials: Overview, in A. Mortensen (ed.), Concise Encyclopedia of Composite Materials (2nd ed.), Elsevier (2007)

[34] K.K. Chawla, Composite Materials - Science and Engineering (2nd ed.), Springer (1998)

[35] C. Zweben, Composite Materials, in M. Kutz (ed.), Mechanical Engineers' Handbook Vol.1: Materials and Materials design (3rd ed.), Wiley \& Sons (2006), Chapter 10

[36] M.Y. He, J.W. Hutchinson, Crack deflection at an interface between dissimilar elastic materials, Int. J. Solids Structures 25 (1989), 1053-1067

[37] D. Koch, K. Tushtev, G. Grathwohl, Ceramic fibre composites: Experimental analysis and modeling of mechanical properties, Composites Science and Technology 68 (2008), 1165-1172

[38] D. Koch, Microstructural modelin and thermomechanical properties, in W. Krenkel (ed.), Ceramic Matrix composites - Fibre reinforced ceramics and their application, Wiley-VCH (2008), Chapter 10

[39] D.B. Marshall, J.B. Davis, Ceramics for future power generation technology: fibre reinforced oxide composite, Current Opinion in Solid State and Materials Science 5 (2001), 283-289

[40] A.G. Evans, F.W. Zok, Review: The physics and mechanics of fibrereinforced brittle matrix composites, J. Mater. Sci. 29 (1994), 3857-96

[41] P.F Becher, Microstructural design of toughened ceramics, J. Am. Ceram. Soc. 74 (1991), 255-269

[42] F.W. Zok, C.G. Levi, Mechanical properties of porous-matrix ceramic composites, Advanced Engineering Materials 3 (2001), 15-23

[43] F.F. Lange, W.C. Tu, A.G. Evans, Processing of damage-tolerant, oxidation resistant ceramic matrix composites by a precursor infiltration and pyrolysis method, Mater. Sci. Eng. A195 (1995), 145-150

[44] C.G. Levi, J.Y. Yang, B.J. Dalgleish, F.W. Zok, A.G. Evans, Processing and performance of an all-oxide ceramic composite, J. Am. Ceram. Soc. 81 (1998), 2077-2086 
[45] M. Schmuecker, P. Mechnich, All-oxide ceramic matrix composites with porous matrices, in W. Krenkel (ed.), Ceramic Matrix composites - Fibre reinforced ceramics and their application, Wiley-VCH (2008), Chapter 9

[46] W.C. Tu, F.F. Lange, A.G. Evans, Concept for damage-tolerant ceramic composites with ,strong“ interfaces, J. Am. Ceram. Soc. 79 (1996), 417424

[47] J.J. Haslam, K.E. Berroth, F.F. Lange, Processing and properties of an alloxide composite with a porous matrix, J. Eur. Ceram. Soc. 20 (2000), 607618

[48] M.A. Mattoni, J.Y. Yang, C.G. Levi, F.W. Zok, Effects of matrix porosity on the mechanical properties of a porous-matrix, all-oxide ceramic composite, Am. Ceram. Soc. 84 (2001), 2594-2602

[49] J. Davidovits, Geopolymers - Inorganic polymeric new materials, J. Thermal. Anal. 37 (1991), 1633-1656

[50] J. Davidovits, Mineral Polymers and methods of making them, US Patent No. 4,349,386 (1982)

[51] M. Sofi, J.S.J. van Deventer, P.A. Mendis, G.C. Lukey, Engineering properties of inorganic polymer concretes (IPCs), Cement and Concrete Research 37 (2007), 251-257

[52] D.M. Roy, Alkali-activated cements - Opportunities and challenges, Cement \& Concrete Research 29 (1999), 249-254

[53] J.G.S. van Jaarsveld, J.S.J. van Deventer, L. Lorenzen, The potential use of geopolymeric materials to immobilise toxic metals: Part I. Theory and Applications, Miner. Eng. 10 (1997), 659-669

[54] P. Duxson, The structure and thermal evolution of metakaolin geopolymers, PhD Thesis, University of Melbourne, Australia (2006)

[55] J.L. Provis, Modeling the formation of geopolymers, PhD Thesis, University of Melbourne, Australia (2006)

[56] P. Duxson, J.L. Provis, G.C. Lukey, S.W. Mallicoat, W.M. Kriven, J.S.J. van Deventer, Understanding the relationship between geopolymer composition, microstructure and mechanical properties, Colloids and Surfaces A: Physicochem. Eng. Aspects 269 (2005), 47-58

[57] M. Steveson, K. Sagoe-Crentsil, Relationships between composition, structure and strength of inorganic polymers, Part I, J. Mater. Sci. 40 (2005), 2023-2036 
[58] K. Komnitsas, D. Zaharaki, Geopolymerisation: A review and prospects for the minerals industry, Miner. Eng. 20 (2007), 1261-1277

[59] F. Pacheco-Torgal, J. Castro-Gomes, S. Jalali, Alkali-activated binders: A review Part. 1. Historical background, terminology, reaction mechanisms and hydration products, Construction and Building Materials 22 (2008), $1305-1314$

[60] P. Duxson, A. Fernandez-Jimenez, J.L. Provis, G.C. Lukey, A. Palomo, J.S.J. van Deventer, Geopolymer technology: the current state of the art, $J$. Mater. Sci. 42 (2007), 2917-2933

[61] K.J.D. MacKenzie, What are these things called geopolymers? A physicochemical perspective, in Advances in Ceramic Matrix Composites IX, Ceramic Transactions 153 (2003), 175-186

[62] J. Davidovits, Geopolymer chemistry and sustainable Development. The Poly(sialate) terminology: a very useful and simple model for the promotion and understanding of green-chemistry, Proceedings of the World Geopolymer 2005 (2005), 9-15

[63] A. Buchwald, What are geopolymer? Current state of research and technology, the opportunities they offer, and their significance for the precast industry, BFT 72 (2006), 42-49

[64] H. Rahier, B. van Mele, M. Biesemans, J. Wastiels, X. Wu, Lowtemperature synthesized aluminosilicate glasses - Part I: Low-temperature reaction stoichiometry and structure of a model compound, J. Mater. Sci. 31 (1996), 71-79

[65] J. Davidovits, Synthetic mineral polymer compound of the silicoaluminates family and preparation process, US Patent No. 4,472,199 (1984)

[66] J. Davidovits, J.L. Sawyer, Early high strength mineral polymer, US Patent No. 4,509,958 (1985)

[67] J. Davidovits, Geopolymers and geopolymeric materials, J. Thermal. Anal. 35 (1989), 429-441

[68] R.A., Fletcher, K.J.D. MacKenzie, C.L. Nicholson, S. Shimada, The composition range of aluminosilicate geopolymers, J. Eur. Ceram. Soc. 25 (2005), 1471-1477 
[69] J.L. Provis, J.S.J. van Deventer, Introduction to geopolymers, in J.L. Provis, J.S.J. van Deventer (ed.), Geopolymers - Structure, processing, properties and industrial applications, Woodhead Publishing Ltd. (2009), Chapter 1

[70] W. Loewenstein, The distribution of aluminium in the tetrahedra of silicates and aluminates, American Mineralogist 39 (1954), 92-96

[71] H. Rahier, B. Wullaert, B. van Mele, Influence of the degree of dehydroxylation of kaolinite on the properties of aluminosilicate glasses, $J$. Thermal. Anal. Cal. 62 (2000), 417-427

[72] H. Xu, J.S.J. van Deventer, The geopolymerisation of alumino-silicate minerals, Int. J. Miner. Process. 59 (2000), 247-266

[73] H. Xu, Geopolymerisation of aluminosilicate Minerals, PhD Thesis, University of Melbourne, Australia (2002)

[74] H. Xu, J.S.J. van Deventer, Geopolymerisation of multiple minerals, Miner. Eng. 15 (2002), 1131-1139

[75] J.G.S. van Jaarsveld, J.S.J. van Deventer, G.C. Lukey, The effect of composition and temperature on the properties of fly ash- and kaolonitebased geopolymers, Chem. Eng. J. 89 (2002), 63-73

[76] P.V. Krivenko, G.Y. Kovalchuk, Direct synthesis of alkaline aluminosilicate minerals in a geocement matrix, J. Mater. Sci. 42 (2007), 2944-52

[77] K.J.D. MacKenzie, S. Komphanchai, R. Vagana, Formation of inorganic polymers (geopolymers) from 2:1 layer lattice aluminosilicates, J. Eur. Ceram. Soc. 28 (2008), 177-181

[78] J.L. Provis, S.L. Yong, P. Duxon, Nanostructure/microstructure of metakaolin geopolymers, in J.L. Provis, J.S.J. van Deventer (ed.), Geopolymers - Structure, processing, properties and industrial applications, Woodhead Publishing Ltd. (2009), Chapter 5

[79] J.W. Phair, J.S.J. van Deventer, Effect of silicate activator $\mathrm{pH}$ on the leaching and material characteristics of waste-based inorganig polymers, Miner. Eng. 14 (2001), 289-304

[80] D.R.M. Brew, K.J.D. MacKenzie, Geopolymers synthesis using silica fume and sodium aluminate, J. Mater. Sci. 42 (2007), 3990-3993

[81] D. Kolousek, J. Brus, M. Urbanova, J. Andertova, V. Hulinsky, J. Vorel, Preparation, structure and hydrothermal stability of alternative (sodium silicate-free) geopolymers, J. Mater. Sci. 42 (2007), 9267-75 
[82] C. Panagiotopoulou, E. Kontori, T. Perraki, G. Kakali, Dissolution of aluminosilicate minerals and by-products in alkaline media, J. Mater. Sci. 42 (2007), 2967-73

[83] H. Rahier, J. Wastiels, M. Biesemans, R. Willlem, G. van Assche, B. van Mele, Reaction mechanism, kinetics and high temperature transformations of geopolymers, J. Mater. Sci. 42 (2007), 2982-2996

[84] L. Weng, K. Sagoe-Crentsil, Dissolution process, hydrolysis and condensation reactions during geopolymer synthesis: Part I - Low Si/ Al ratio systems, J. Mater. Sci. 42 (2007), 2997-3006

[85] A. Palomo, M.T. Blanco-Varela, M.L. Granizo, F. Puertas, T.Vazquez, M.W. Grutzeck, Chemical stability of cementitious materials based on metakaolin, Cement \& Concrete Research 29 (1999), 997-1004

[86] C.E. White, J.L. Provis, A. Llobet, T. Proffen, J.S.J. van Deventer, Evolution of local structure in geopolymer gels: an in-situ neutron pair distribution function analysis, J. Am. Ceram. Soc. 94 (2011), 3532-3539

[87] J.L. Provis, G.C. Lukey, J.S.J. van Deventer, Do Geopolymers actually contain nanorystalline Zeolites? A reexamination of existing results, Chem. Mater. 17 (2005), 3075-3085

[88] M. Schmücker, K.J.D. MacKenzie, Microstructure of sodium polysialate siloxo geopolymer, Ceramics International 31 (2005), 433-437

[89] V.F.F. Barbosa, K.J.D. MacKenzie, Thermal behaviour of inorganic geopolymers and composites derived from sodium polysialate, Mater. Res. Bull. 38 (2003), 319-331

[90] V.F.F. Barbosa, K.J.D. MacKenzie, C. Thaumaturgo, Synthesis and characterisation of materials based on inorganic polymers of alumina and silica: sodium polysialate polymers, International Journal of Inorganic polymers 2 (2000), 309-317

[91] M.R. Rowles, J.V. Hanna, K.J. Pike, M.E. Smith, B.H. O'Connor, ${ }^{29} \mathrm{Si}$, ${ }^{27} \mathrm{Al},{ }^{1} \mathrm{H}$ and ${ }^{23} \mathrm{Na}$ MAS NMR study of the bonding character in aluminosilicate inorganic polymers, Appl. Magn. Reson. 32 (2007), 663689

[92] R. Brooks, M. Bahadory, F. Tovia, H. Rostami, Properties of alkaliactivated fly ash: high performance to lightweight, International Journal of Sustainable Engineering 3 (2010), 211-218

[93] H. Wang, H. Li, F. Yan, Synthesis and mechanical properties of metakaolinite-based geopolymer, Colloids and Surfaces A: Physicochem. Eng. Aspects 268 (2005), 1-6 
[94] M.L. Granizo, M.T. Blanco-Varela, S. Martinez-Ramirez, Alkali activation of metakaolins: parameters affecting mechanical, structural and microstructural properties, J. Mater. Sci. 42 (2007), 2934-2943

[95] R.R. Lloyd, Accelerated ageing of geopolymers, in J.L. Provis, J.S.J. van Deventer (ed.), Geopolymers - Structure, processing, properties and industrial applications, Woodhead Publishing Ltd. (2009), Chapter 8

[96] V.F.F. Barbosa, K.J.D. MacKenzie, Synthesis and thermal behaviour of potassium sialate geopolymers, Materials Letters 57 (2003), 1447-1482

[97] J.L. Bell, P.E. Driemeyer, W.M. Kriven, Formation of ceramics from metakaolin-based geopolymers. Part I - Cs-based geopolymers, J. Am. Ceram. Soc. 92 (2009), 1-8

[98] J. Davidovits, Ceramic-ceramic composite material and production method, US Patent No. 4,888,311 (1989)

[99] J. Davidovits, Alkaline aluminosilicate geopolymeric matrix for composite materials with fibre reinforcement and method for obtaining same, US Patent No. 5,798,307 (1998)

[100] O. Bortnovsky, J. Dedecek, Z. Tvaruzkova, Z. Sobalik, J. Subrit, Metal ions as probes for characterization of geopolymer materials, J. Am. Ceram. Soc. 91 (2008), 3052-3057

[101] S.J. O’Connor, K.J.D. MacKenzie, M.E. Smith, J.V. Hanna, Ion exchange in the charge-balancing sites of aluminosilicate inorganic polymers, J. Mater. Chem. 20 (2010), 10234-10240

[102] P. Sazama, O. Bortnovsky, J. Dedecek, Z. Tvaruzkova, Z. Sobalik, Geopolymer based catalysts - New group of catalytic materials, Catalysis Today 164 (2001), 92-99

[104] B.G. O’Leary, K.J.D. MacKenzie, unpublished results, Victoria University of Wellington, 2012 